The uptake of carbon sources by Aspergillus niger

Jasper Sloothaak 


\section{Thesis committee}

\section{Promotor}

Prof. Dr V.A.P. Martins dos Santos

Professor of Systems and Synthetic Biology

Wageningen University \& Research

\section{Co-promotors}

Dr P.J. Schaap

Associate professor, Laboratory of Systems and Synthetic Biology

Wageningen University \& Research

Dr J.A. Tamayo-Ramos

Postdoctoral researcher, International Research Center in Critical Raw Materials for Advanced Industrial Technologies (ICCRAM), University of Burgos, Spain

\section{Other Members}

Dr S. Hartmans, DSM, Delft, the Netherlands

Prof. Dr W.J.H. van Berkel, Wageningen University \& Research

Prof. Dr M. Penttillä, VTT Technical Research Centre, Espoo, Finland

Prof. Dr B.J. Zwaan, Wageningen University \& Research

This research was conducted under auspices of the Graduate School VLAG (Advanced studies in Food Technology, Agrobiotechnology, Nutrition and Health Sciences) 


\title{
The uptake of carbon sources by Aspergillus niger
}

\author{
Jasper Sloothaak
}

\section{Thesis}

submitted in fulfilment of the requirements for the degree of doctor

$$
\text { at Wageningen University }
$$

by the authority of the Rector Magnificus

Prof. Dr A.P.J. Mol,

in the presence of the

Thesis Committee appointed by the Academic Board

to be defended in public

on Friday the $9^{\text {th }}$ of June 2017

at 11 a.m. in the Aula. 
Jasper Sloothaak

The uptake of carbon sources by Aspergillus niger, 189 pages.

$\mathrm{PhD}$ thesis, Wageningen University, Wageningen, the Netherlands (2017) With references, with summary in English

ISBN 978-94-6343-208-5

DOI http://dx.doi.org/10.18174/414746 


\section{Propositions}

1. The design of a successful microbial cell factory must include substrate import, especially when we are not feeding it with its favorite meal. (this thesis)

2. Design of synthetic transporters should be preceded by a full characterization of nature's own examples (this thesis)

3. Experts now have a better chance at correctly predicting the substrate specificity of a transporter than the outcome of elections

4. The CRISPR-Cas9 technology is the Rosetta stone for functional genomics

5. Fracking can boost methanogenic bioconversion

6. No organisms have saved as many human lives as fungi

7. News is based on provenance, fake news gains it afterwards

8. Stubbornness combined with reflection is a great cocktail to overcome obstacles in life

Propositions belonging to the thesis, entitled

The uptake of carbon sources by Aspergillus niger

Jasper Sloothaak

Wageningen, $9^{\text {th }}$ of June 2017 


\section{TABLE OF CONTENTS}

Chapter 1

Thesis Summary

Chapter 2

General Introduction

Chapter 3 68

Aspergillus niger membrane-associated proteome analysis for the identification of glucose transporters

Chapter 4

Identification and functional characterization of novel xylose transporters from the cell factories Aspergillus niger and Trichoderma reesei

Chapter 5 98

Overexpression of the Aspergillus niger GatA transporter leads to preferential use of D-galacturonic acid over D-xylose

Chapter 6

Identification of a novel L-rhamnose uptake transporter in the filamentous fungus Aspergillus niger

Chapter 7

General Discussion

Acknowledgements

About the author

List of publications

177

Overview of completed training activities 
Chapter 1

Thesis summary 


\section{Thesis summary}

\section{Summary}

Fungi have been used as food and in food fermentations long before written accounts were created and they have been used in folk medicine in ancient cultures. For centuries, species of the genus Aspergillus have been used for the preparation of traditional Asian foodstuffs or together with baker's yeast in preparation of alcoholic beverages and have therefore been of great economical value. Later, Aspergillus niger has been used for large-scale production of organic acids, enzymes and other food-additives. Today, we aim to harness its saprophytic nature and extraordinary ability to degrade and utilize plant material that is naturally recalcitrant to degradation. To facilitate that ability, the range of sugar transporters employed by this fungus is large even among fungi. This makes it an excellent choice for the identification and characterization of a variety of proteins with different substrate specificities, with potential application in the design of newly engineered cell factories. At the same time this diversity and complexity makes it very challenging to understand the role of each of the components of the sugar uptake transporting network and regulatory mechanisms involved.

In chapter 2, the current energy and material problems that need to be faced in the coming decades are discussed. A growing world population with increased prosperity in the developing countries stresses our resources and the environment. Using renewable second-generation biomass feedstocks can partly fill in the energy demand and significantly reduce the demand for chemicals from petrochemical origin. To be able to efficiently utilize these renewable resources, we need to understand and control the uptake of sugars released from biomass feedstock into our microbial cell factories. Methods for studying these transporters and the different sugar transporter families are discussed.

In chapter 3, the application of plasma-lemma proteomics and the use of profile Hidden Markov Models (HMM) is introduced, where the proteome of the cellular membrane is isolated, the differential expression of surface proteins is determined and their possible role as glucose transporters is analyzed. Aspergillus niger was cultured with various monomeric sugars as sole carbon source and subjected to proteomics analysis (LC-MS/MS) after membrane enrichment and 
purification. Membrane protein abundances were determined, and those scoring high with the $\mathrm{HMM}_{\text {glut }}$ model (generated using 42 biochemically characterized glucose transporters from 10 different organisms), and displaying specific expression patterns indicating a possible role on glucose transport, were selected for their functional validation and characterization. Two candidate transporters, MstG and MstH, were selected and their function was confirmed by functional complementation in a glucose-transporter null yeast strain. Their transport characteristics for various monomeric sugars were determined, their transcriptional regulation was analyzed, and it was concluded that the selected candidates were indeed two new Aspergillus niger high-affinity glucose transporters.

In chapter 4, xylose transporters from the enzyme producers Aspergillus niger and Trichoderma reesei are studied. To identify candidate transporters, we took advantage of in silico proteome mining and specific profile HMM model predictions. For their functional validation and characterization, we took advantage of a yeast expression host unable to transport monosaccharides, and able to metabolize xylose. The six selected candidates XltA, B and C from $A$. niger and Str1, 2 and 3 from $T$. reesei were heterologously expressed, and all of them supported growth of the expression host on xylose. Transcript analysis and radiometric sugar uptake assays revealed specificity for xylose for $A$. niger XltA and $\mathrm{XltB}$, suggesting specific biological roles for these transporters in xylose uptake in $A$. niger. New insights were also gained into the molecular mechanisms regulating the pentose utilization, at inducer uptake level, in these fungi.

In chapter 5, the endogenous carbon catabolite repression system is exploited to demonstrate the function of the $A$. niger galacturonic acid transporter GatA. In a mixed sugar fermentation, it was found that the A. niger strain overexpressing GatA preferred D-galacturonic acid over D-xylose as substrate, and an increased uptake of D-galacturonic acid was observed. Also, an elevated activity of the Dgalacturonic acid reductase activity was found, suggesting that the endogenous D-galacturonic acid catabolic pathway is metabolite controlled.

In chapter 6, the first eukaryotic L-rhamnose transporter was identified and functionally validated. A comparative plasmalemma proteomic analysis was used to identify candidate L-rhamnose transporters in A. niger. Further analysis was focused on one protein, designated RhtA, which was classified as a Fucose: $\mathrm{H}+$ Symporter (FHS) from the TC2.A.1.7. transporter family. This family previously 
included functionally validated transporters exclusively from bacterial origin able to use different sugars and deoxysugars, but not rhamnose. Indications for L-rhamnose transport function were obtained by functional complementation in combination with growth inhibition tests of the $S$. cerevisiae mutant strain EBY.VW4000. Biochemical analysis using radiolabeled L-rhamnose confirmed that RhtA is a L-rhamnose transporter. The RhtA gene was found to be located in tandem with a hypothetical alpha-L-rhamnosidase gene (rhaB). Transcriptional analysis of $r h t A$ and $r h a B$ in wild type and deletion strains confirmed that both genes have a coordinated expression, being strongly and specifically induced by L-rhamnose, and regulated by the RhaR, which controls tightly the expression of genes involved in rhamnose utilization.

In Chapter 7, the work described in this thesis is summarized and its impact for society and future research are discussed. Furthermore, insights gained in this thesis, related to regulatory mechanisms, protein structure and evolution are discussed. 
Chapter 2

General introduction 


\section{General Introduction}

\section{Societal embedding and the need for better understanding of transporters}

\section{Scope}

To significantly reduce our dependency on fossil fuels in favor of biotechnological solutions, new generation of high-potential technologies and insights encouraging successive improvements are required to produce innovative biobased products that will be highly valued in the marketplace. The work presented in this thesis is funded by the BE-Basic Foundation, an international public-private partnership aiming at industrial biobased solutions for a sustainable society, and focuses on the identification, regulation analysis and characterization of sugar transporters essential for the utilization of second generation feedstocks, as well as on their potential for application in the design of novel cell factories.

This thesis was performed in the scope of the 'carbon-based compounds' flagship, which focuses on the conversion of lignocellulosic materials (first \& second generation) and other bio-based feedstocks (glycerol, alcohols, acids and furanics), including their contaminants, into relevant products.

\section{Towards a sustainable society}

The success of human society has always been heavily dependent on availability of food. The change from hunting gathering towards agriculture of cattle and crops supported the first great population increase. After this transition, populations could increase to up to 100 -fold before reaching stability, making us dependent on high yield food production. During the modern industrial and agricultural revolution, we have been able to increase life expectancy and further increase agricultural yields, and the world population has exploded since [1] (Figure 1). The United States Census Bureau estimated that the world population reached 1 billion people in 1804 and it took 123 years to reach 2 billion in 1927. Global population reached 7 billion in 2012, an increase of 5 billion people in 88 years [2]. This last revolution was mainly possible due to the utilization of fossil resources (coal, oil and natural gas). After food, human society is now largely dependent on the materials facilitating our lives and the energy required to produce and power them. Among the different fossil fuels, oil has fueled transport vehicles, provided us with many of those chemicals and materials, and has therefore empowered global economic growth and prosperity for more than 
100 years. But three major problems are inherent to our dependence of oil. Firstly, the availability of crude oil is finite. The natural carbon deposits were formed from the organic material of the autotrophs that populated the earth before any heterotrophs had evolved. Natural forces converted this accumulated material over the course of millions of years to the abundant energy source

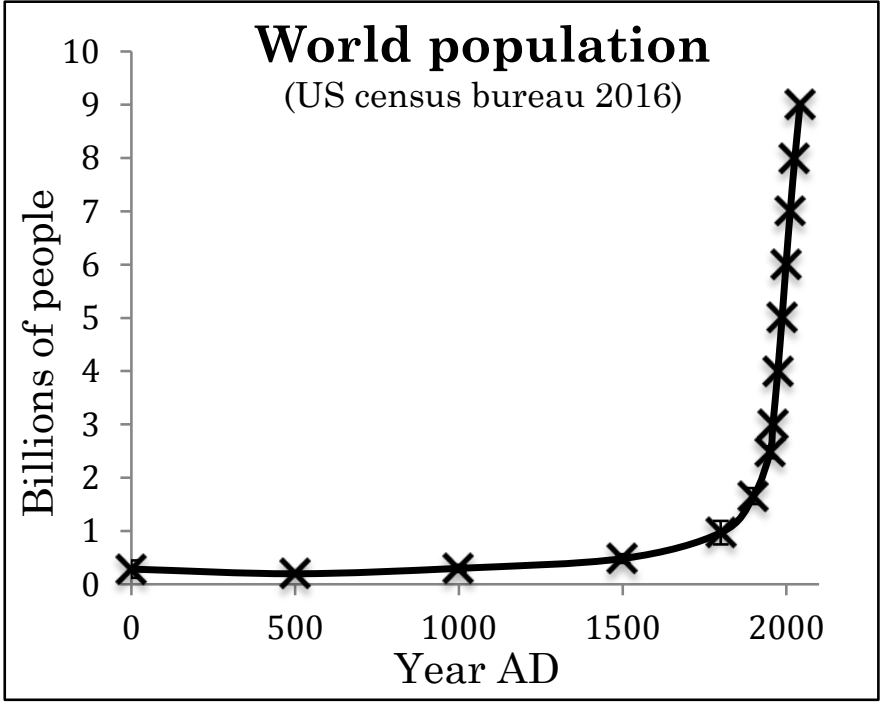

Figure 1: Historic World Population as estimated by the US census bureau in 2016 we now retrieve from the earth. The organic material that is currently formed by autotrophs is not deposited, but in most cases directly used by heterotrophs, like us, and is thereby returned to the atmosphere as CO2. Just as when heterotrophs burn carbohydrates for energy, the use of oil in our society results in the release of large quantities of $\mathrm{CO} 2$ in the atmosphere. These enormous quantities released together with methane emissions from livestock contribute to greenhouse effect and change the climate [3]. While heavily disputed for many decades, this second problem is now generally accepted. A third problem is the political power that comes from the control of the geographical areas where oil deposits are present and the military struggle that arises from it. Since our economy is based on monetary trust, instability of energy supply always leads to some sort of recession. The examples are plenty, well known are the events related to the first and second gulf war.

\section{Sustainable energy sources}

There are plenty of incentives to come up with sustainable alternative sources for our energy, chemicals and materials. Extensive data on energy use and production is available for Europe by Eurostat (ec.europe.eu/eurostat) and for the United States from the US Energy Information Administration (EIA) (www.eia.gov). According to the EIA approximately $75 \%$ of all crude oil used is applied in heating and transportation. Therefore, alternative and renewable energy sources have been a major research topic for the past decades. These 
efforts have led to development of the technologies for nuclear fission power, geothermal power, hydropower, solar power, wind power and biomass power. Each of these technologies has its advantages and drawbacks. Nuclear fission is a very powerful and seemingly cheap technology that does not contribute to climate change through greenhouse gas emissions. However, it is based on deposited uranium, so not sustainable. Furthermore, the waste generated by the fission technology is still highly radioactive and very dangerous for thousands of years to any organisms exposed. Geothermal, hydro-, solar, wind and biomass power are renewable and do not contribute to climate change, but cannot yet satisfy the energy demands. Due to government incentives, the shares of renewable energy sources are increasing in US, Europe and South America [4][5][6]. Undoubtedly, this is also the case in other countries, where data is not available. In Europe, biomass power is rapidly growing and has taken up the second biggest share of renewable energy after hydropower [5]. It is of interest because of four additional benefits. One, while the other power systems generate electricity, which is liable to losses for storage and transport, biomass is used to produce energy carriers that don't have these losses. Two, making energy carriers such as ethanol or biodiesel gives an added benefit; the energy carriers can be 'plugged' into in the existing transportation infrastructure. Energy expenditure for transport is roughly $30 \%$ of total expenditure, and while a small part of our transportation is now electrically driven, the majority is still dependent on combustion engines. Three, carbon is fixed in biomass production, this means that burning the resulting energy carriers does not result in net $\mathrm{CO}_{2}$ release. Finally, the one property of biomass over other energy sources is that it can be used for production of chemicals, and thus materials.

\section{Biomass for bioenergy}

When considering biomass as substrate for energy we need to make a clear distinction. Wood biomass has been used until the mid-1800's as the main energy source for heating, cooking and light. While the introduction of petroleum based fuels made a big impact on that, many households and smaller ventures (hotels, restaurants etc.) still use wood as an important source for heating. The biomass present in waste has traditionally also been used as fuel in conventional thermal power stations, or more recently in modern power plants. This makes for a very significant contribution to our energy production. These applications all relate to the direct burning, action or pyrolysis of the biomass to run generators that generate electricity. The references to biomass in this thesis, however, only relate to the use of biomass as a substrate for biochemical conversion. In that sense, 
biomass has originally been used in the form of edible (also named 'first generation') sugars, competing with food sources.

The first-generation sugars have been used to develop the technology for production of bioethanol fuel. The US and Brazil have made themselves partly independent of fossil fuels using biomass fermentation technologies. These countries have implemented government policies for energy safety to stimulate production and use of corn or sugar cane derived bioethanol. The infrastructure for production and distribution is fully functional and operational. Since the start of these governmental agricultural and technological incentives, there has been controversy about the effect on food and fuel prices, the environment and economy in general. A thorough meta-data study is now available that combines all available data on the effect of the implementation of corn-derived ethanol in the US [7]. It is found that the effect of corn derived ethanol fuel on food and fuel prices, environment and overall economy is limited. Policies were originally made to increase energy security, but positive effects on national economy or the environment would greatly enhance support for this technology. The discussion still revolves around the availability of edible crops and the competition of fuel production versus food production. Also, cultivating crops only for conversion to ethanol greatly reduces the positive impact of the replacement of petroleum based fuels. The introduction of lignocellulosic ethanol technology starts to solve these problems. Here, the waste products of food production are used and therefore the food-fuel competition is removed. Furthermore, the food crops themselves are more efficiently used and no additional efforts are done that negatively contribute to the environment. Current technologies, however, are not yet commercially feasible and need improvements in production yields. As mentioned before, other energy carriers can be created by fermentation technology using (lignocellulosic) biomass as substrate, such as butanol or methanol. Several ventures are advanced in their research for the commercial production of these alternative biofuels as can be deduced from several patents [8].

\section{Biomass for biochemicals}

The production of bioenergy carriers made from biomass is a very interesting and large problem. The running cost of a biotechnological production process is relatively large compared to the running cost of a wind generator, hydropower

plant or solar panel (www.irena.org). Corn or sugarcane ethanol production costs are already competitive with gasoline for several years, but when considering the agricultural residue lignocellulose as substrate, production costs are much higher and therefore not competitive with gasoline prices [9]. Value of biomass products is often depicted in the value pyramid, where the size of the layer is the market 
volume of the product and the vertical location of the layer represents the value of the product (Figure 2). The largest and lowest value step of the pyramid can increasingly be filled with renewable electricity sources as previously mentioned. Biomass is therefore the alternative substrate of choice for some of the other steps of the pyramid, where no alternatives are available. In the near future, the large developing countries China, India and Brazil are likely to reach a level of prosperity to match those of the US and Europe. The demand for affordable automobiles, homes, smart electronics for communication and entertainment, healthy nutrition and cosmetics are, therefore, expected to rise [10]. Novel methods and technologies for the efficient and sustainable production of these advanced materials and solutions that do not compete with food production are required. Companies are currently in advanced stages of research and application for many biochemicals, employing genetically modified microorganisms for their production, such as DSM (ethanol and succinic acid), Corbion (lactic acid), Butamax (Butanol) and many others.

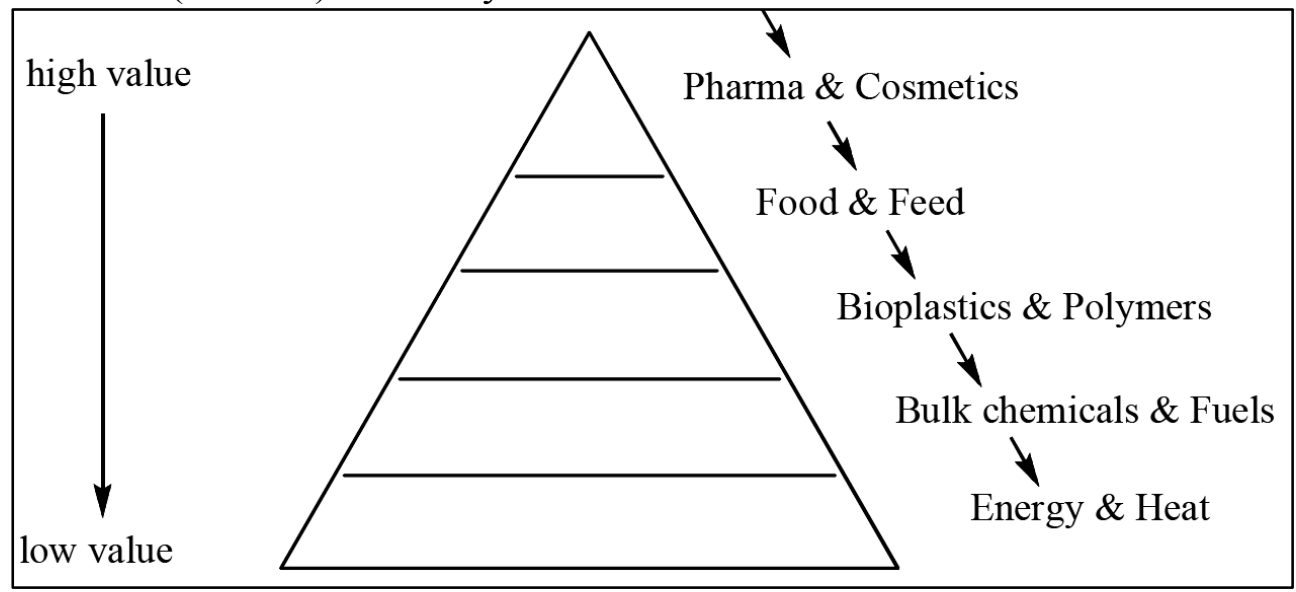

Figure 2: The value pyramid

\section{Composition of plant biomass}

Microorganisms take sugars and convert them to biochemicals via metabolic routes. The type of sugars that can be used is dependent on the microorganism used, and the type of sugars available is dependent on the type of biomass used. The first generation biomass substrates that were industrially applied are sugar cane derived sucrose, a disaccharide of fructose and glucose, and corn derived starch, a polymer of glucose. Fructose and glucose are released as monomers by the activity of a few enzymes, and can be readily used by the yeast Saccharomyces cerevisiae to produce ethanol or by Aspergillus niger to produce organic acids. 
Lignocellulose is the second-generation biomass substrate that is currently most widely studied. It is the main constituent of residues of many crops used for food or feed production. There are three very abundant second-generation feedstocks, rich in lignocellulose, that are currently the most studied targets for secondgeneration biochemical production: the residues of corn production after harvesting of the kernels (the leaves and stem called stover); the residue of sugar cane (the fibrous material left after sugar extraction called bagasse); and the residue after removal of the wheat and rice kernels (straw). In addition, there are many other sources such as grasses, woods, but also aquatic species such as algae and kelp, which are also considered of interest as second-generation feedstocks. Lignocellulose consists of a network of three different polymers, with a composition of $40-50 \%$ cellulose, $30-40 \%$ hemicellulose and around $20 \%$ lignin [11]. Cellulose and hemicellulose are polysaccharides of glucose and xylose respectively that can be enzymatically degraded to monomers. Lignin is an amorphous recalcitrant polymer that is generally not considered fermentable and is therefore burned for energy after removal. There is, however, research done on chemical conversion of the lignin polymer to create useful chemicals, which was recently reviewed [12].

Pectin is also a second-generation feedstock component that is not yet as widely considered as substrate for biochemical production. It is a major constituent of the agricultural residues of the production of beet sugar, fruit juices, potato starch and other types of biomass, as seen in figure 3. The composition of these feedstocks is similar to that of lignocellulose, but the lignin is replaced by pectin [13]. The polymer consists mainly of the sugaracid galacturonic acid (galA), with a high degree of esterification with acetyl and methyl groups, and neutral sugar side-groups. The most abundant forms of pectin are

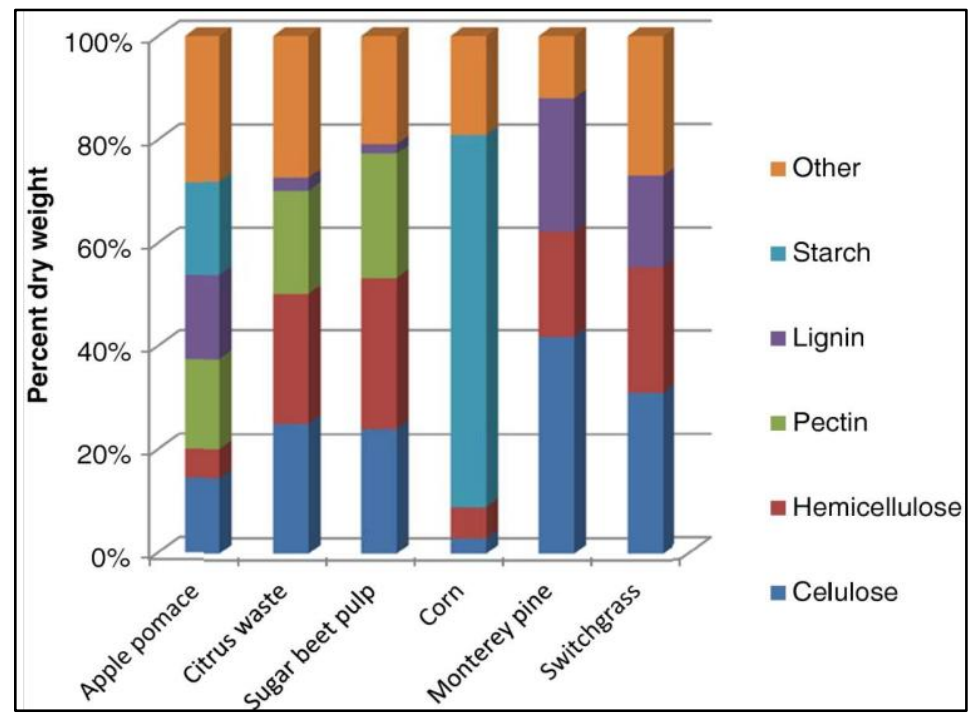

Figure 3: Comparison of the dry weight composition of pectin rich biomass to starches and lignocellulosic biomass 
homogalacturonan (HG), where the backbone consists solely of galacturonic acid units, rhamnogalacturonan I (RGI), where the backbone consists of alternating units of galacturonic acid and rhamnose, and rhamnogalacturonan II (RGII), a highly branched and substituted form of HG. The sugars that can be derived by enzymatic hydrolysis of pectins are galacturonic acid, rhamnose, arabinose, xylose, galactose and others present in minor amounts. This structural variability and diversity of sugar components is the scientific challenge that must be addressed to efficiently use pectin as a second-generation feedstock.

\section{Relevance of transporters for fermentation}

The biological conversion of renewable biomass substrates to biochemicals by fermentation requires roughly three steps. The first step consists of three parts, which are very much dependent on the type of feedstock that is used and the robustness of the organism that will be used in the bioconversion. Generally, the pre-treatment consists of a mechanical disruption to open up the dense structure of the plant material to make it accessible. This is followed by a chemical pretreatment step, involving heat, pressure, acid or base. Then by an enzymatic hydrolysis step, where enzymes (usually of fungal origin) release the monomeric sugars from the polymers. In the second step, microbial conversion, the substrate is converted into a product. The third step involves downstream processing in which the product of interest is isolated and purified from the fermentation broth, cells are recovered and the product is separated from the water and other soluble impurities based on its chemical characteristics.

In the second step, when the feedstock is fed to the microbial cell-factories, the sugars enter the cells through the action of transporter proteins and are converted inside the cell to a product that is usually actively transported out of the cell by other transporter proteins. Each individual transporter protein has a specificity and intrinsic uptake rate towards a sugar substrate. As this sugar uptake rate can be limiting in high performance processes, tailor-made, optimal transporters will be required. In organisms that can naturally utilize the sugars in the feed, endogenous regulatory systems will control the induction of transporters to optimize substrate uptake over a range of in vivo conditions. For instance, in the yeast $S$. cerevisiae, there are 20 transporters known that can transport glucose into the cell. In vitro and in industrial conditions, a number of these transporters are also able to import xylose, arabinose or galactose, each with a different affinities and uptake rates, even though the yeast cells are unable to metabolize these sugars directly $[14,15]$. These transporters show different affinities to each of these sugars and will be selective towards a single substrate when a mixture of sugars is present. In S. cerevisiae, when glucose is present, xylose is normally 
not transported, even though the transporter is able to take it up in a homogeneous xylose solution. This poses a problem when using complex sugar mixtures, because in order to compete with chemical alternatives, productivity and titres should be maximized, and fermentation times should be minimized. Therefore, the industry aims to utilize organisms that are able to transport all substrates at the same time. To be able to customize the suite of transporters that is available for substrate uptake, a library of transporters with known characteristics must be available. Many bacteria are limited in the number of substrates that can be metabolized, and prokaryotic transporter proteins might not function in eukaryotic production hosts. When considering eukaryotes, the model organism $S$. cerevisiae can naturally only metabolize glucose and fructose and therefore only has transporters that are optimized for the uptake of these particular sugars. Saprophytic fungi such as $A$. niger, however, can metabolize almost any sugar derived from lignocellulosic and pectic feedstocks, such as xylose and rhamnose, and has the optimal transporters for the uptake of those sugars. To facilitate the engineering of production strains for efficient uptake of feedstock-derived sugars, this thesis, focussed on the identification and characterization novel fungal sugar transporters. 


\section{Tools for identification and characterization of transporters}

\section{Identification of LacY}

The group of Monod published the very first description of a membrane transporter in 1956 of the galactoside permease LacY [16]. The researchers studied uptake by Escherichia coli of $\mathrm{S}^{35}$-analogs of galactosides, which cannot be metabolized, by measuring the radioactivity of cells after incubation. Cells that had been exposed to lactose prior to incubation with the labeled substrate showed an internal concentration of more than a 100 fold of that in the medium. Cells that were not exposed did not show any significant accumulation. This is probably the first account of differential expression that is described in literature, which gave the basis for the idea of coordinated expression of related genes and led to the discovery of the bacterial operon concept. This discovery was well before the start of 'the genome era': the overexpression of the LacY coding gene was first reported in 1978 and the sequence described in 1980 [17,18]. Currently, we have many other tools available that can be employed to identify transporter proteins and subsequently characterize them.

\section{Genomic localization and homology}

In the past, transporters were sometimes found by expression of a random genomic library or screening of genomic library with a probe [19,20]. Alternatively, when several catalytic genes of a metabolic pathway are localized in an operon, the importer of the substrate of that pathway could be at that same genomic location [21]. Since today we often have a genome sequence available, or can generate it in a matter of weeks, we use bioinformatics to predict coding sequences and associated protein sequence. A telling example of the development of technology is the identification of FucP, the fucose importer of E. coli. The genes responsible for the metabolism of fucose and their co-regulation were elucidated between 1956 and 1977 [22-25]. The FucP sequence was then reported in 1987 but functional expression was only reported in 1994 [21,26]. In that time, while functionality of FucP was experimentally validated, technology for automated alignment of DNA and protein sequences became available so that other transporters like XylE and AraE were identified and functionally characterized in parallel as reviewed by Henderson et al. in 1990 [27-29]. When transporters from very diverse organisms had been identified, it became clear that many of them had a number of domains that showed a high level of amino acid sequence similarity [30]. This is the case because they all possess a number of hydrophobic protein domains that allow them to permeate the cellular membrane. The precise location of these membrane-spanning $\alpha$-helices can be predicted by 
studying the characteristics of the amino acids in the protein with a secondary structure prediction software, such as tmHMM [31]. TmHMM based secondary structure predictions together with profile-HMM built from protein alignmentand homology-based annotations were crucial in filtering the data sets obtained from transcriptomics or proteomics. In chapter 3, the application of profileHidden Markov Models (HMM) for homology search is compared with the classic BLAST algorithm search, demonstrating the superiority of the former method [32].

\section{romics techniques to study differential expression}

When research on bacterial transporters started, no complete genome sequence data was available and therefore a transport function could only be coupled to a physiological condition and not the functional unit $[16,33]$. Later, the responsible genomic locus could be identified by the introduction of linkage studies where marker functions were coupled to random mutagenesis. Tailored screening approaches led to construction of the first knockout strains and finally to the identification of transporter genes by functional complementation, as was the case for AraE [28]. Currently, differential expression analysis can be exploited, making use of wild type and targeted knockout strains in combination with transcriptomics or proteomics approaches, to get insights into the possible role of genes. Discriminating conditions are chosen such that it is expected that genes of interest are differentially expressed, and the $\sim$ omics techniques allow us to look at a snapshot of the whole mRNA pool (transcriptomics) or the whole protein pool (shotgun proteomics). For transcriptomics, condition-specific RNA is isolated from cells. Differential expression of individual RNAs within the RNA pool is either studied by microarray or by shotgun sequencing of the library of RNA molecules (RNA sequencing). For shotgun proteomics, complete pools of proteins are isolated from the discriminating conditions, or from specific part of the organism such as membranes or mitochondria. The composition of the protein pool and relative protein abundances are then analyzed using a combination of liquid chromatography (LC) and mass-spectrometry (MS). Both of these data types then require further processing using bioinformatics tools to answer specific questions related to expression. These techniques are specifically important when looking for distantly related transporters such as from diverse families as can be seen in chapter 6 .

\section{Functional validation}

Functional validation must follow initial identification of candidate sugar transporters. In general, five methods can be distinguished. The easiest method 
is the overexpression of the transporter in the endogenous host that has a functional transport system. Changes in apparent affinity or uptake rate can then be measured and linked to the specific function of the transporter. This method does not give direct evidence for the in vivo function of the transporter and experiments may be needed to gather additional experimental evidence, as seen in chapter 5 and in Sauer et al. 1999 [20]. Moreover, experimental conditions where overexpression of the transporter leads to a clearly distinctive phenotype may be hard to find, and this method is therefore not generally applicable.

Alternatively, the transporter of interest can be deleted or disrupted in the endogenous host, and a lack-of-function can be determined by a quantification of the resulting phenotype such as a reduced affinity for the substrate and substrate uptake rate. The success of this method is dependent on the redundancy of the transporter function in the host, and may require additional experiments. In prokaryotes, this method has proven successful, but eukaryotes tend to show (apparent) functional redundancy and resilience with regard to sugar uptake as described in chapter 6 and previously by others $[28,14]$.

A third method focussing on the transporter in isolation requires the expression of the transporter of interest, ex vivo or in vitro. Ex vivo; in a host that lacks the transporter function, naturally or through genetic engineering, or in vitro; in phospholipid vesicles that are reconstituted from purified membranes from the host that lacks the transport function. In the former, simple growth on metabolizing sugar can be used to confirm the functionality of the candidate sugar transporter as described in chapters 3, 4 and 5, and in Weierstall et al. 1999 [19]. The latter is more tedious to accomplish, but has advantages such as of intracellular accumulation due to the lack of metabolizing enzymes, possibility to study bidirectional transport using inside out vesicles and the study of organelle specific transporters [34-36]. Ex vivo expression is often sufficient for the study of membrane sugar transporters, as is employed in this thesis. The expression of the transporter can then be coupled to one of the following four methods to determine function or detailed transport parameters. The function of electrogenic transporters can be measured using voltage- or patch clamp methods [37]. The function of sugar/proton symporters can be measured by $\mathrm{pH}$ measurements [21]. The function of any transporter can be measured by uptake of radiolabeled substrates or the application of in vivo fluorescent sensors for sugars [38,39].

\section{Families of transporters with sugar uptake function}

A comprehensive system has been developed to classify transporters based on their basic mechanism of action and how they use energy to power that action. 
This transporter classification system is available online (www.TCDB.org), is curated and based on cumulative evidence [40-42]. Some major groups of transporters can be distinguished that have members capable of transporting sugars. These families are described here.

\section{Major Facilitator Superfamily TC 2.A.1}

Transporters of the Major Facilitator Superfamily 2.A contains two families of transporters with sugar transporter capacities. One of these families is the family TC2.A.1 and shares the name with family 2.A. These transporters are part of the 'secondary active' transporters that use the free energy of a gradient to drive the transport of their substrate. For transport of substrate sugars across the fungal membrane this is the only relevant transporter superfamily. It is also the largest transporter superfamily occurring in all known cells with high structural, mechanistic and substrate diversity encompassing 58 families, with 6 families that contain proteins with known sugar transporting function as can be seen in table 1. Many of the best-studied sugar transporters belong to this superfamily. The previously described LacY, XylE and AraE of E. coli and the human GLUT1 are a few of those that have been studied in detail over the past decades [43]. Numerous studies have focused on this (super-) family of transporters, and the 3D structure of some of them has been elucidated in all possible states of the protein. In general, the MFS transporters function as a monomer, are approximately 500 amino acids in size, encoding for 12 transmembrane helices (TMH). Due to this topology both the $\mathrm{C}$ and $\mathrm{N}$ terminus reside in the cytosol. Several exceptions exist; one family has $14 \mathrm{TMHs}$, one protein has $24 \mathrm{TMHs}$ and one protein has only 6 TMHs. Within this superfamily three distinct mechanisms for transport have been observed; uniport, symport and antiport, each using the membrane gradient of the (co-)substrate to drive the function [44]. Each transporter class shows a high degree of conservation within the families but show very weak similarity between the families in the superfamily. The superfamily is characterized by two small conserved sequences between helices 2 and 3, and between helices 8 and 9 [30]. This reflects an apparent structural symmetry between the 6 helices at either end of the protein, and suggests that they have evolved from a functional dimer. Upon binding of the (co-) substrate, the $\mathrm{N}$ - and $\mathrm{C}$-terminal domains move relative to each other, opening the substrate binding domain to either the cytoplasmic or periplasmic side of the membrane.

Table 1 Sugar-specific families within the major facilitator superfamily (MFS)

\begin{tabular}{|l|l|}
\hline TC no. & Family \\
\hline 2.A.1 & The major facilitator superfamily (MFS) \\
\hline 2.A.1.1 & The sugar porter (SP) family \\
\hline
\end{tabular}




\begin{tabular}{|l|l|}
\hline 2.A.1.2 & The drug: $\mathrm{H}+$ antiporter drug efflux family (DHA12) \\
\hline 2.A.1.5 & The oligosaccharide: $\mathrm{H} 1$ symporter $(\mathrm{OHS})$ family \\
\hline 2.A.1.7 & The fucose: $\mathrm{H} 1$ symporter (FHS) family \\
\hline 2.A.1.20 & The sugar efflux transporter (SET) family \\
\hline
\end{tabular}

\section{Glycoside-pentoside-hexuronide:cation symporter family TC 2.A.2}

The other family in the Major Facilitator Superfamily that contains sugar transporters is the $\mathrm{CPH}$ family. These transporters mostly catalyse the uptake of glycoside sugars in symport with a monovalent cation such as $\mathrm{Na}+, \mathrm{H}+$ or $\mathrm{Li}$. Members of this family have been identified and characterized from bacteria and from eukaryotes such as Schizosaccharomyces, Arabidopsis and human [45-47]. The proteins are generally about 500 amino acids in length, with twelve transmembrane helices. There is some structural variation in this family, because lactose permeases from the Gram-positive bacteria have an additional C-terminal hydrophilic domain that is interacting with the phosphotransferase system like the PTS transporters from superfamily 4.A [48]. Furthermore, indications are found for an ability to form functional homodimers, resulting in a double sugar translocation route [49]. This family, however, does not contribute to the uptake of sugars as substrate for metabolism in fungi.

\section{Drug/metabolite transporter superfamily TC 2.A.7}

This superfamily consists of 35 families, each characterized by function, size and topology [50,51]. The RhaT family TC 2.A.7.6 includes 2 rhamnose:H+ symporters of E. coli and Salmonella typhimurium, both of which have been functionally characterized and are of specific interest for this thesis [52]. The RhaT proteins of both species are 344 amino acids long with 10 putative TM domains. Other families in this superfamily transport a very diverse range of substrates such as nucleotide-sugar transporters in the membranes of the ER and golgi of eukaryotic cells [53].

\section{Solute:sodium symporters. TC 2.A.21}

The first sugar transporters identified are the mammalian Glucose Transporters, identified from studies on the kidney and intestine [54]. Here they are responsible for the uptake of glucose over the brush border cell membranes of the enterocytes. SGLT1 couples the import of one glucose molecule to the import of $2 \mathrm{Na}^{+}$ molecules, and has relatively high affinity with a $\mathrm{Km}$ of $0.2 \mathrm{mM}$. SGLT2 couples the import of glucose to the import of $1 \mathrm{Na}+$ molecule, and has a relatively low affinity with $\mathrm{Km}$ of $6 \mathrm{mM}$. To keep homeostasis, ATP-dependent pumps control the $\mathrm{Na}^{+}$current over the membrane. Therefore, the combined functions of these two transporters indirectly couple the import of glucose to the availability of ATP 
in the cells. SGLT1 and 2 are members of a group of 6 human SGLT's that can all transport glucose and several other sugars with the exception the sensor SGLT3. These SGLT's are of major importance for human health care, because they have been associated to several genetic diseases and are therefore drug targets. Many transporters have been identified that use the sodium gradient as a driving force to transport a variety of solutes. They have an amino acid chain length of approximately 600 residues, with 14 predicted transmembrane helices (TMH) and a conserved core of 10 TMH's and a conserved substrate binding location. The only SSS transporter characterized in fungi, the Saccharomyces cerevisiae DUR3, conserved in several filamentous species such as Aspergillus niger, is active on urea $[55,56]$. It is therefore unlikely that the SSS transporters play a role in sugar transport in fungi.

\section{SWEET; Sugars Will Eventually be Exported Transporter. TC 2.A.123}

The first transporters of the SWEET family were identified by heterologous expression of an Arabidopsis gene library in combination with a FRET sugar biosensor in mammalian cells [39]. In the Arabidopsis genome, 17 SWEET proteins can be found, that have been studied with respect to substrate, by heterologous expression in yeast. They can be separated in four clades and transport several mono- and disaccharides, such as sucrose, glucose, galactose and fructose [57]. They consist of 7 transmembrane helices of which the Cterminal three are mirrored in the N-terminal three, with an inverting helix in between the two triplets. This topology is believed to be evolved from the ancestor of the prokaryotic semiSWEETS, where the functional unit is a dimer of a protein with only three trans-membrane spanning helices [58]. Because the activity of these SWEETS seems to be independent of $\mathrm{pH}$ it was concluded that they function as uniporters. These specific SWEET transporters function in transport of sugars over cell membranes to distribute the carbon source, generated by photosynthesis in the leaves, to the other locations in the plant. They are of specific interest because plant pathogens have been shown to specifically upregulate several of these efflux transporters during infection. This effect is crucial for effective infection, because inhibition of this induction confers resistance of the plant to the pathogen. SWEETs have been identified in the genomes of the rice and potato plant, but also in metazoan (animal) cells. Since animals lack a photosynthetic system, the function as it was postulated for the plant SWEETs is irrelevant. In the human genome only one SWEET transporter is found, that localizes to the golgi apparatus and is able to transport glucose. It was believed that the MFS transporter GLUT mediates uptake and efflux of glucose, but null-mutations in GLUT do not lead to defects related to impaired 
efflux [39]. Therefore, it is possible that the SWEET transporter has that function in mammals. Furthermore, in mice it was found that this gene is upregulated in mammary cells during lactation, when glucose is converted to lactose in the golgi and exported to the milk. Chen, 2010 suggested that the human SWEET could be responsible for transport of glucose from our intestinal cells to our bloodstream via vesicular efflux [39]. Therefore, this transporter is now an interesting target for research in obesity and diabetes. While it is suggested that SWEETS are susceptibility factors of plants for fungi, no fungal SWEET proteins have been identified to date. The proteins are however functional in fungal cells as was demonstrated by the functional heterologous expression of glucose transporting SWEETS in yeast cells [39]. This makes these small proteins an interesting target for industrial application in microbial cell factories.

\section{ATP binding cassette family TC 3.A.1}

These transporters are part of the primary transporters, using the energy of ATP hydrolysis to power their function. The first $\mathrm{ABC}$ transporters, published in 1982, are the $\mathrm{ABC}$ maltose transporters that facilitate import of maltose in the human pathogen $S$. typhimurium or the human intestinal symbiont $E$. coli [59]. These first transporters were characterized as 'periplasmic protein dependent' or 'binding protein dependent, because they require a periplasmic protein or receptor for proper functioning. This auxiliary protein was suggested to be involved in transport over the outer membrane of gram-negative cells [60] (Ames, 1986). Later it was found that a Substrate Binding Protein (SBP), in the periplasmic space of gram-negative bacteria or externally for archaea and gram positive bacteria, delivers the substrate to the transporter complex. Also, in eukaryotes several $\mathrm{ABC}$ import systems have been identified that were originally classified according to a characterizing feature, in the case of P-glycoproteins, or function, in the case of multidrug resistance, leading to some ambiguity in literature. Since the discovery of the common ATB Binding Cassette domain $(\mathrm{ABC})$ in all these functionally diverse families, the characterization that is currently accepted is based on that feature. Thanks to modern advanced crystallography, much of their structural diversity is elucidated. In general, they can be characterized as importers or exporters and based on their structural diversity, the importers are further divided into three classes; Type I, Type II and the ECF transporters Type III [61]. However, an exception to the unidirectional nature of the $\mathrm{ABC}$ transporters was discovered, suggesting that this might not be an overall common feature, and other energy systems can power membrane transport [62]. This is the second biggest family of transporters described thus far, likely because up to $3 \%$ of the currently sequenced genomes of bacteria and 
archaea code for these transporters [63]. These transporters span a huge substrate range and only a very small fraction of the family functions as sugar transporters, characterized as Type I importers. Furthermore, the importers of Type I, II and III identified to date are only found in prokaryotes. The ABC exporters can also be found in eukaryotes, but no sugar transport function has been found for those. The ABC transporter consists of two times two functional domains that are covalently linked or expressed as separate proteins or combinations thereof. Two permease or Trans Membrane Domains (PMDs) are required, consisting of 6 transmembrane alpha helices each, which form the substrate translocation pore in the membrane. On the cytoplasmic site of the membrane, two ATP/Nucleotide Binding Cassettes (NDBs) are attached to conserved sequences in the binding loops of the PMDs [64].

\section{Phosphoenolpyruvate Transport System TC 4.A}

These transporters are part of the group translocators that couple the chemical modification of the substrate to the transport over the cell membrane. The phosphoenolpyruvate (PEP) transport system was first identified in E. coli in 1964 as a result of the search for a homologous system to the eukaryotic kinase involved in sialic acids metabolism [65]. The system was found to be diverse with respect to substrates and only a small fraction is involved in sugar transport, summarized in Table 2. In contrast to several other families, these transporters consist of several functional protein subunits. These units can be covalently linked, but are in many cases multimer complexes. The protein complex effectively catalyses several phosphoryl transfer steps, transferring the phosphoryl group to the transported solute. The first part of the PTS consists of two cytosolic proteins that are responsible for the energy coupling and are pathway specific. The first of those proteins, called enzyme I (EI), is auto phosphorylated by PEP. EI then phosphorylates the second protein, a phosphoryl transferase called Histidine Protein or Heat stable Protein (HPr). The second part of the PTS, enzyme II (EII), is permease specific and consists of 3 functional units $\mathrm{A}, \mathrm{B}$ and $\mathrm{C}$. EIIA is phosphorylated by $\mathrm{HPr}$ and subsequently phosphorylates EIIB. EIIB then finally phosphorylates the substrate that is transported over the membrane by permease domain EIIC. The EIIC permease domain is used as the basis for the classification of the PTS subfamilies [66]. The PTS system is interwoven with regulatory systems related to both central nitrogen and central carbon metabolism. As an example, in E. coli, the phosphorylation state of the cytosolic pools of the EI and HPr components is correlated with the pool of cyclic Adenosine MonoPhosphate (cAMP) via the activity of adenylate cyclase. 
Table 2 Families of sugar importers of the bacterial phosphotransferase system (PTS) superfamily.

\begin{tabular}{|l|l|}
\hline TC no. & Family \\
\hline 4.A.1 & The PTS glucose-glucoside (Glc) family \\
\hline 4.A.2 & The PTS fructose-mannitol (Fru) family \\
\hline 4.A.3 & The PTS lactose-N,N'-diacetylchitobiose--3-glucoside (Lac) family \\
\hline 4.A.4 & The PTS glucitol (Gut) family \\
\hline 4.A.5 & The PTS galactitol (Gat) family \\
\hline 4.A.6 & The PTS mannose-fructose-sorbose (Man) family \\
\hline
\end{tabular}

cAMP is a steric inducer of the CRP transcription factor that regulates core carbon metabolism, thereby coupling the sugar transport to the central carbon metabolism [67]. The PTS system is found in bacteria and archaea, but no examples have been found in eukarya.

\section{D.2 The Glycosyl Transferase 2 (GT2) Family}

These membrane proteins are believed to be involved in extracellular glycosylation by catalyzing the attachment of a hydrophobic carrier to a sugar molecule. Although suggested, it is not sure if these proteins are involved directly in the transport of sugars [68]. Most consist of several monomers, together about 8 - TMHs plus a C-terminal hydrophilic domain of approximately 340 amino acids harbours the catalytic activity. N-terminal domains show homology with some of the ABC transporter families (3.A.3.4.3 and 4), and the C-terminal domain shows homology with one of the MFS families (2.A.1.3.43). Members have only been identified in Archaea and Bacteria.

\section{Concluding remarks}

Transporter proteins are as diverse as the molecules that are transported over the cellular membranes. Computational tools have helped develop a comprehensive classification system providing insights in the functionality of shared domains. For many functional proteins the identification of a catalytic domain is often sufficient to correctly predict the function. With catalytic proteins, a chemical reaction is catalyzed and the substrate is converted to a chemically different product, so that the combination of several active site residues is required in a specific spatial arrangement. Transporters have residues for binding and transferring the substrate across the membrane, but the entire structure of the protein is required for the conformational changes that constitute the function. Primary sequence alignments are therefore of limited value in predicting the characteristics and substrates of transporters. As is showcased in this thesis, 
characterization of transporters can therefore be laborious and cannot yet be executed in a high-throughput manner. The knowledge on characteristics and substrates of prokaryotic and eukaryotic transporters is thus limited in contrast to the wealth of uncharacterized transporters that can be detected using computational tools and algorithms. Transporters are, however, the in- and outputs of microbial cell factories, and can no longer be neglected in the design of novel pathways for fermenting natural renewable feedstocks to virtually any biobased chemical of human interest. The knowledge and insights obtained in this thesis and other similar research is therefore of crucial importance in the development of the next generation of synthetic microbial cell factories. In the current society where oil dominates the chemical and energy market and drastically affects the environment adversely, we desperately need competitive industrial processes to provide a sustainable alternative. This thesis focuses on the identification and characterization of novel sugar transporters essential for application in the design of novel cell factories. 


\section{References}

1. Diamond J. Guns, germs and steal: the fates of human societies. Perspectives in Biology and Medicine. 1999.

2. United States Census Bureau. Population Projections [Internet]. Census.gob. 2013. p. National Population Projections Data. Available: http://www.census.gov/population/projections/

3. Pachauri RK, Meyer LA. IPCC, 2014: Climate Change 2014: Synthesis Report. Contribution of Working Groups I, II and III to the Fifth Assessment Report of the Intergovernmental Panel on Climate Change [Internet]. IPCC, Geneva, Switserland. 2014. doi:10.1073/pnas.1116437108

4. U.S. Energy Information Administration. Annual Energy Outlook 2015. Office of Integrated and International Energy Analysis. 2015. doi:DOE/EIA-0383(2013)

5. Eurostat. Energy balance sheets. [Internet]. Publications Office of the European Union. 2016. doi:10.2785/52802

6. Cremonez PA, Feroldi M, Feiden A, Gustavo Teleken J, José Gris D, Dieter J, et al. Current scenario and prospects of use of liquid biofuels in South America. Renewable and Sustainable Energy Reviews. 2015. pp. 352-362. doi:10.1016/j.rser.2014.11.064

7. Hochman G. Corn Ethanol and US Biofuel Policy Ten Years Later : A Systematic Review and Meta-analysis Corn Ethanol and US Biofuel Policy Ten Years Later : A Systematic Review and Meta-analysis. Agric Appl Econ Assoc Annu Meet. 2016; 1-48. Available: http://purl.umn.edu/235467

8. Donaldson GK, Eliot AC, Flint D, Maggio-Hall LA, Nagarajan V. Fermentive production of four carbon alcohols. US Pat .... 2010;8178328: 1-101. doi:http://www.google.com/patents/US7851188

9. Foley T, Thornton K, Hinrichs-rahlwes R, Sawyer S, Sander M, Taylor $\mathrm{R}$, et al. Renewables 2015. Global status report. Bloomberg; 2015.

10. Deloitte and VNCI. The Chemical Industry in the Netherlands: World leading today and in 2030-2050. Deloitte Netherlands. 2012;

11. Haghighi Mood S, Hossein Golfeshan A, Tabatabaei M, Salehi Jouzani G, Najafi GH, Gholami M, et al. Lignocellulosic biomass to bioethanol, a comprehensive review with a focus on pretreatment. Renew Sustain Energy Rev. 2013;27: 77-93. doi:10.1016/j.rser.2013.06.033

12. Upton BM, Kasko AM. Strategies for the Conversion of Lignin to HighValue Polymeric Materials: Review and Perspective. Chem Rev. American Chemical Society; 2016;116: 2275-2306. 
doi:10.1021/acs.chemrev.5b00345

13. Edwards MC, Doran-Peterson J. Pectin-rich biomass as feedstock for fuel ethanol production. Applied Microbiology and Biotechnology. 2012. pp. 565-575. doi:10.1007/s00253-012-4173-2

14. Wieczorke R, Krampe S, Weierstall T, Freidel K, Hollenberg CP, Boles E. Concurrent knock-out of at least 20 transporter genes is required to block uptake of hexoses in Saccharomyces cerevisiae. FEBS Lett. 1999;464: 123-128. doi:10.1016/S0014-5793(99)01698-1

15. Apel AR, Ouellet M, Szmidt-Middleton H, Keasling JD, Mukhopadhyay A. Evolved hexose transporter enhances xylose uptake and glucose/xylose co-utilization in Saccharomyces cerevisiae. Sci Rep. Nature Publishing Group; 2016;6.

16. Rickenberg H V, Cohen G N BG\& MJ. La galactoside-perméase d'Escherichia coli. Ann Inst Pasteur. 1956;91: 829-57. Available: papers2://publication/uuid/D5751144-5FF2-4A65-97FF-E1099B805F81

17. Teather RM, Müller-Hill B, Abrutsch U, Aichele G, Overath P. Amplification of the lactose carrier protein in Escherichia coli using a plasmid vector. MGG Mol Gen Genet. Springer-Verlag; 1978;159: 239248. doi:10.1007/BF00268260

18. Büchel DE, Gronenborn B, Müller-Hill B. Sequence of the lactose permease gene. Nature. 1980;283: 541-5. Available: http://www.ncbi.nlm.nih.gov/pubmed/6444453

19. Weierstall T, Hollenberg CP, Boles E. Cloning and characterization of three genes (SUT1-3) encoding glucose transporters of the yeast Pichia stipitis. Mol Microbiol. 1999;31: 871-83. Available: http://www.ncbi.nlm.nih.gov/pubmed/10048030

20. Sauer N, Friedländer K, Gräml-Wicke U. Primary structure, genomic organization and heterologous expression of a glucose transporter from Arabidopsis thaliana. EMBO J. European Molecular Biology Organization; 1990;9: 3045-50. Available: http://www.ncbi.nlm.nih.gov/pubmed/2209537

21. Bradley SA, Tinsley CR, Muiry JAR, Henderson PJF. Proton-linked Lfucose transport in Escherichia coli. Biochem J. 1987;248: 495-500.

22. Green M, Cohen SS. Enzymatic Conversion of L-Fucose to L-Fuculose. J Biol Chem. 1956;219: 557-568.

23. Heath EC, Ghalambor MA. The Metabolism of 1-Fucose : I. THE PURIFICATION AND PROPERTIES OF 1-FUCULOSE KINASE. J Biol Chem. 1962;237: 2423-2426. Available: http://www.jbc.org/content/237/8/2423.short 
24. Hacking AJ, Lin ECC. Disruption of the fucose pathway as a consequence of genetic adaptation to propanediol as a carbon source in Escherichia coli. J Bacteriol. 1976;126: 1166-1172.

25. Hacking AJ, Lin ECC. Regulatory changes in the fucose system associated with the evolution of a catabolic pathway for propanediol in Escherichia coli. J Bacteriol. 1977;130: 832-838.

26. Gunn FJ, Tate CG, Henderson PJ. Identification of a novel sugar-H+ symport protein, FucP, for transport of L-fucose into Escherichia coli. Mol Microbiol. 1994;12: 799-809. Available: http://www.ncbi.nlm.nih.gov/pubmed/8052131

27. Davis E0, Jones-Mortimer MC, Henderson PJF. Location of a Structural Gene for Xylose-H+ Symport at 91 Min on the Linkage Map of Escherichia coli K12. J B1OLOGICAL Chem (c. 1984;259: 1520-1525.

28. MacPherson AJ, Jones-Mortimer MC, Henderson PJ. Identification of the AraE transport protein of Escherichia coli. Biochem J. Portland Press Ltd; 1981;196: 269-83. Available: http://www.ncbi.nlm.nih.gov/pubmed/7030324

29. Henderson PJF, Maiden MCJ. Homologous Sugar Transport Proteins in Escherichia coli and Their Relatives in Both Prokaryotes and Eukaryotes. Philos Trans R Soc B Biol Sci. 1990;326: 391-410. doi:10.1098/rstb.1990.0020

30. Maiden MCJ, Davis EO, Baldwin SA, Moore DCM, Henderson PJF. Mammalian and bacterial sugar transport proteins are homologous [Internet]. Nature. Nature Publishing Group; 1987. pp. 641-643. doi:10.1038/325641a0

31. Krogh A, Larsson B, von Heijne G, Sonnhammer ELL. Predicting transmembrane protein topology with a hidden markov model: application to complete genomes1. J Mol Biol. 2001;305: 567-580. doi:http://dx.doi.org/10.1006/jmbi.2000.4315

32. Altschul SF, Gish W, Miller W, Myers EW, Lipman DJ. Basic local alignment search tool. J Mol Biol. 1990;215: 403-410. doi:http://dx.doi.org/10.1016/S0022-2836(05)80360-2

33. NOVOTNY C, ENGLESBERG E. The 1-arabinose permease system in Escherichia coli B/r. Biochim Biophys Acta - Gen Subj. 1966;117: 217230. doi:10.1016/0304-4165(66)90169-3

34. Kasahara M, Hinkle PC. Reconstitution and Purificaton of the D-Glucose Transporter from Human Erythrocytes. J Biol Chem. 1977;252: 73847390.

35. Baldwin JM, Lienhard GE, Baldwin SA. The monosaccharide transport 
system of the human erythrocyte. Orientation upon reconstitution. Biochim Biophys Acta - Biomembr. Elsevier; 1980;599: 699-714. doi:10.1016/0005-2736(80)90211-4

36. Palmieri F, Agrimi G, Blanco E, Castegna A, Di Noia MA, Iacobazzi V, et al. Identification of mitochondrial carriers in Saccharomyces cerevisiae by transport assay of reconstituted recombinant proteins. Biochimica et Biophysica Acta - Bioenergetics. 2006. pp. 1249-1262. doi:10.1016/j.bbabio.2006.05.023

37. Loo DDF, Hirayama BA, Karakossian MH, Meinild A-K, Wright EM. Conformational dynamics of hSGLT1 during $\mathrm{Na}+$ /glucose cotransport. $\mathrm{J}$ Gen Physiol. 2006;128: 701-20. doi:10.1085/jgp.200609643

38. Walsh MC, Smits H-P, van Dam K. Respiratory inhibitors affect incorporation of glucose into Saccharomyces cerevisiae cells, but not the activity of glucose transport. Yeast. John Wiley \& Sons, Ltd.; 1994;10: 1553-1558. doi:10.1002/yea.320101204

39. Chen L-Q, Hou B-H, Lalonde S, Takanaga H, Hartung ML, Qu X-Q, et al. Sugar transporters for intercellular exchange and nutrition of pathogens. Nature. Nature Research; 2010;468: 527-532. doi:10.1038/nature09606

40. Pao SS, Paulsen IT, Saier MH. Major facilitator superfamily. Microbiol Mol Biol Rev. 1998;62: 1-34. Available:

http://www.pubmedcentral.nih.gov/articlerender.fcgi?artid=98904\&tool= pmcentrez\&rendertype $=$ abstract

41. Saier MH, Tran C V, Barabote RD. TCDB: the Transporter Classification Database for membrane transport protein analyzes and information. Nucleic Acids Res. 2006;34: D181-6. doi:10.1093/nar/gkj001

42. Saier MH. The Transporter Classification Database. Recent Adv nucleic acid Res. 2016;44: 372-9.

43. Bell GI, Kayano T, Buse JB, Burant CF, Takeda J, Lin D, et al. Molecular biology of mammaliam glucose transporters. Diabetes Care. American Diabetes Association; 1990;13: 198-208. doi:10.4052/tigg.4.99

44. Law CJ, Maloney PC, Wang D. Ins and Outs of Major Facilitator Superfamily Antiporters. Annu Rev Microbiol. 2008; 289-305. doi:10.1146/annurev.micro.61.080706.093329.Ins

45. Reinders A, Ward JM. Functional characterization of the $\alpha$-glucoside transporter Sut 1p from Schizosaccharomyces pombe, the first fungal homologue of plant sucrose transporters. Mol Microbiol. Blackwell 
Science Ltd; 2001;39: 445-454. doi:10.1046/j.1365-2958.2001.02237.x

46. Stadler R, Truernit E, Gahrtz M, Sauer N. The AtSUC1 sucrose carrier may represent the osmotic driving force for anther dehiscence and pollen tube growth in Arabidopsis. Plant J. 1999;19: 269-278.

doi:10.1046/j.1365-313X.1999.00527.x

47. Bassik MC, Kampmann M. Knocking out the door to tunicamycin entry. Proc Natl Acad Sci U S A. 2011;108: 11731-2. doi:10.1073/pnas.1109035108

48. Rodríguez-Díaz J, Rubio-del-Campo A, Yebra MJ. Lactobacillus casei ferments the N-Acetylglucosamine moiety of fucosyl- $\alpha-1,3-\mathrm{N}-$ acetylglucosamine and excretes L-fucose. Appl Environ Microbiol. 2012;78: 4613-4619. doi:10.1128/AEM.00474-12

49. Veenhoff LM, Heuberger EHML, Poolman B. The lactose transport protein is a cooperative dimer with two sugar translocation pathways. EMBO J. 2001;20: 3056-3062. doi:10.1093/emboj/20.12.3056

50. Jack DL, Yang NM, Jr MHS. The drug / metabolite transporter superfamily. 2001;3639: 3620-3639.

51. Västermark $\AA$, Almén MS, Simmen MW, Fredriksson R, Schiöth HB. Functional specialization in nucleotide sugar transporters occurred through differentiation of the gene cluster EamA (DUF6) before the radiation of Viridiplantae. BMC Evol Biol. BioMed Central; 2011;11: 123. doi:10.1186/1471-2148-11-123

52. Tate CG, Muiry JAR, Henderson PJF. Mapping, cloning, expression, and sequencing of the rhaT gene, which encodes a novel L-rhamnose-H+ transport protein in Salmonella typhimurium and Escherichia coli. J Biol Chem. American Society for Biochemistry and Molecular Biology; 1992;267: 6923-6932. Available: http://www.ncbi.nlm.nih.gov/pubmed/1551902

53. Song Z. Roles of the nucleotide sugar transporters (SLC35 family) in health and disease. Molecular Aspects of Medicine. 2013. pp. 590-600. doi:10.1016/j.mam.2012.12.004

54. Murer H, Hopfer U. Demonstration of electrogenic Na+-dependent Dglucose transport in intestinal brush border membranes. Proc Natl Acad Sci U S A. 1974;71: 484-8. Available:

http://www.pubmedcentral.nih.gov/articlerender.fcgi?artid=388031\&tool $=$ pmcentrez\&rendertype $=$ abstract

55. Sumrada R, Gorski M, Cooper T. Urea Transport-Defective Strains of Saccharomyces cerevisiae. J Bacteriol. 1976;125: 1048-1056.

56. ElBerry HM, Majumdar ML, Cunningham TS, Sumrada RA, Cooper 
TG. Regulation of the urea active transporter gene (DUR3) in Saccharomyces cerevisiae. J Bacteriol. 1993;175: 4688-4698.

57. Eom J-S, Chen L-Q, Sosso D, Julius BT, Lin I, Qu X-Q, et al. SWEETs, transporters for intracellular and intercellular sugar translocation. Curr Opin Plant Biol. 2015;25: 53-62. doi:10.1016/j.pbi.2015.04.005

58. Tao Y, Cheung LS, Li S, Eom J-S, Chen L-Q, Xu Y, et al. Structure of a eukaryotic SWEET transporter in a homotrimeric complex. Nature. 2015;527: 259-263. doi:10.1038/nature15391

59. Gilson E, Higgins CF, Hofnung M, Ferro-Luzzi Ames G, Nikaido H. Extensive homology between membrane-associated components of histidine and maltose transport systems of Salmonella typhimurium and Escherichia coli. J Biol Chem. American Society for Biochemistry and Molecular Biology; 1982;257: 9915-8. Available: http://www.ncbi.nlm.nih.gov/pubmed/7050111

60. Ames GFL. Bacterial Periplasmic Transport Systems: Structure, Mechanism, and Evolution. Annu Rev Biochem. Annual Reviews 4139 El Camino Way, P.O. Box 10139, Palo Alto, CA 94303-0139, USA ; 1986;55: 397-425. doi:10.1146/annurev.bi.55.070186.002145

61. ter Beek J, Guskov A, Slotboom DJ. Structural diversity of ABC transporters. J Gen Physiol. 2014;143: 419-435. doi:10.1085/jgp.201411164

62. Balakrishnan L, Venter H, Shilling RA, Van Veen HW. Reversible transport by the ATP-binding cassette multidrug export pump LmrA: ATP synthesis at the expense of downhill ethidium uptake. J Biol Chem. 2004;279: 11273-11280. doi:10.1074/jbc.M308494200

63. Tomii K, Kanehisa M. A comparative analysis of ABC transporters in complete microbial genomes. Genome Res. 1998;8: 1048-1059. doi:10.1101/gr.8.10.1048

64. Wilkens S. Structure and mechanism of ABC transporters. F1000Prime Rep. 2015;7: 14. doi:10.1016/j.sbi.2004.06.005

65. Kundig W, Ghosh S, Roseman S. Phosphate Bound To Histidine in a Protein As an Intermediate in a Novel Phospho-Transferase System*. Proc Natl Acad Sci U S A. 1964;52: 1067-1074. doi:10.1073/pnas.52.4.1067

66. Saier MH. MicroReview Families of transmembrane sugar transport proteins. Mol Microbiol. 2000;35: 699-710.

67. Deutscher J, Francke C, Postma PW. How phosphotransferase systemrelated protein phosphorylation regulates carbohydrate metabolism in bacteria. Microbiol Mol Biol Rev. 2006;70: 939-1031. 
doi:10.1128/MMBR.00024-06

68. Ardiccioni C, Clarke OB, Tomasek D, Issa H a, von Alpen DC, Pond HL, et al. Structure of the polyisoprenyl-phosphate glycosyltransferase GtrB and insights into the mechanism of catalysis. Nat Commun. Nature Publishing Group; 2016;7: 10175. doi:10.1038/ncomms10175 


\section{Chapter 3}

\section{Aspergillus niger membrane-associated proteome analysis for the identification of glucose transporters}

J. Sloothaak†, D. I. Odoni ${ }^{\dagger}$, L. H. de Graaff, V. A. P. Martins dos Santos, P. J. Schaap and J. A. Tamayo-Ramos

\footnotetext{
${ }^{\dagger}$ These authors contributed equally to this work

Biotechnology for Biofuels (2015) Vol 8
} 


\title{
Aspergillus niger membrane-associated proteome analysis for the identification of glucose transporters
}

\begin{abstract}
The development of biological processes that replace the existing petrochemicalbased industry is one of the biggest challenges in biotechnology. Aspergillus niger is one of the main industrial producers of lignocellulolytic enzymes, which are used in the conversion of lignocellulosic feedstocks into fermentable sugars. Both the hydrolytic enzymes responsible for lignocellulose depolymerization and the molecular mechanisms controlling their expression have been well described, but little is known about the transport systems for sugar uptake in A. niger. Understanding the transportome of $A$. niger is essential to achieve further improvements at strain and process design level. Therefore, this study aims to identify and classify $A$. niger sugar transporters, using newly developed tools for in silico and in vivo analysis of its membrane-associated proteome.

In the present research work, a hidden Markov model (HMM), that shows a good performance in the identification and segmentation of functionally validated glucose transporters, was constructed. The model (HMMgluT) was used to analyze the $A$. niger membrane-associated proteome response to high and low glucose concentrations at a low $\mathrm{pH}$. By combining the abundance patterns of the proteins found in the $A$. niger plasmalemma proteome with their HMMgluT scores, two new putative high-affinity glucose transporters, denoted MstG and $\mathrm{MstH}$, were identified. MstG and MstH were functionally validated and biochemically characterized by heterologous expression in a $S$. cerevisiae glucose transport null mutant. They were shown to be a high-affinity glucose transporter $(\mathrm{Km}=0.5 \pm 0.04 \mathrm{mM})$ and a very high-affinity glucose transporter $(\mathrm{Km}=0.06 \pm 0.005 \mathrm{mM})$, respectively.

This study, focusing for the first time on the membrane-associated proteome of the industrially relevant organism A. niger, shows the global response of the transportome to the availability of different glucose concentrations. Analysis of the $A$. niger transportome with the newly developed HMMgluT showed to be an efficient approach for the identification and classification of new glucose transporters.
\end{abstract}




\section{Background}

The development of biological systems for the industrial synthesis of biofuels and chemicals is a main objective of today's biotechnology. For cost-effective production of valuable products, efficient microbial fermentations of non-food lignocellulosic material are essential. Filamentous fungi, in particular the fungus Aspergillus niger, play a prominent role in this field of biotechnology. A. niger is of significant industrial relevance and has been exploited as a production platform for both organic acids and hydrolytic enzymes [1]. It is an efficient degrader of the major plant cell wall polysaccharides cellulose, hemicellulose and pectin [2], and is one of the main industrial producers of commercial enzymes for plant biomass conversion due to its high enzyme secretory capacity [3]. In the last decades, its versatile arsenal of extracellular enzymes for lignocellulose depolymerization [4], and the molecular mechanisms controlling their expression, have been well described [5-7]. However, while previous studies have revealed the existence of an array of uptake systems in this fungus [8-10], little is known about the identity and specificity of the transport systems involved in sugar uptake. Most of the existing knowledge related to monosaccharide uptake in fungi originates from studies in the model yeast Saccharomyces cerevisiae. This yeast is able to transport and metabolize glucose, fructose, mannose and galactose. Transport of these simple sugars is mediated only through facilitated diffusion by the majority of the transporters from the Hxt family, composed of Hxt1-Hxt17, and Gal2. They belong to the sugar porter (SP) family [11], which is the largest subfamily of the major facilitator superfamily (MFS). Hxt1-Hxt4, Hxt6 and Hxt7 have been found to be able to support growth of yeast in glucose on their own, thus being considered the major hexose transporters in yeast. In addition to the Hxt family, three members of the maltose transporter family (Agt1, Mph2 and Mph3) are also able to transport glucose [12]. The individual characterization of each of these transporters was possible using engineered $S$. cerevisiae strains, deleted for hxt1-7 [13] and hxt1-17, gal2, agt 1, mph2 and mph 3 [12], as hosts for the functional validation and biochemical study of these proteins. These yeast mutant strains, unable to grow on glucose, fructose, mannose and galactose as a single carbon source, have also subsequently been used as tools for the functional characterization of sugar transporters from other fungal species [14-21]. In contrast to $S$. cerevisiae, the only functionally validated sugar transporters in $A$. niger are the recently identified D-galacturonic acid transporter GatA [22,23], two fructose transporters [10] and the high-affinity sugar/ $\mathrm{H}_{+}$symporter MstA [14]. Furthermore, transcriptional data for the A. niger mstC gene suggests that it encodes a lowaffinity glucose transporter [9], but no experimental data supporting its role as a 
functional sugar transporter is publicly available. MFS proteins display a strong structural conservation [24], and structure-based profile hidden Markov models can be used to identify putative sugar transporters in the A. niger in silico proteome. To obtain a profile hidden Markov model (HMM), a multiple sequence alignment is turned into a position-dependent scoring system with segments of variable conservation levels and length [25]. As a result of the weighted scoring system, HMMs are less sensitive to changes in the non-conserved regions of a given protein family than more traditional methods based on shared primary sequence similarity alone, like e.g. the standard Blast algorithm. These changes include variability of the residues at a certain position as well as insertions and deletions. In this study, a profile hidden Markov model specific for glucose transporters (HMMgluT) was computed based on a structure-based multiple sequence alignment of 42 proteins with a known function related to glucose transport. Genome information combined with transcriptome analyzes of various growth conditions can give a good inventory of $A$. niger plasma membrane components with hypothetical sugar transporter functions. These data types, however, do not take regulatory events at the posttranscriptional level into account, although these events can influence protein abundances and localization. An inventory of the $A$. niger plasma membrane proteome at defined culture conditions can provide a more reliable source of information for the identification of the most important sugar transport components. To date, only few fungal plasmalemma (PM)-enriched proteomes have been reported, and none of them involved an industrially relevant filamentous fungus. The main focus of study has been on S. cerevisiae [26-28] and on several pathogenic fungi [29-31], as their PM represents a cellular component of substantial interest from a diagnostic and therapeutic point of view [29]. Thus, since the PM proteome of $A$. niger has not been a subject of study yet, it would be a first step towards a better understanding of its dynamics and topology. Recently, our research group successfully used a shotgun proteomics approach to study protein secretion mechanisms in $A$. niger, allowing the characterization of the secretory subproteome of the fungus and its changes in different conditions by using labelfree LC-MS/MS [32,33]. This is a powerful tool to analyze enriched organelle cell fractions, both in qualitative and quantitative terms [34], and thus permits the identification and quantification of the most relevant components of the $A$. niger cell membranes. In this work, the membrane-associated proteome of $A$. niger was studied for the identification of new glucose transporters, using newly developed experimental and complementary computational approaches. 


\section{Results and discussion}

\section{In silico transportome analysis and construction of a hidden Markov model specific for glucose transporters}

As an integral part of the membrane, transporters must contain at least one protein domain that is thermodynamically stable in the hydrophobic environment of the phospholipid tails. In eukaryotes, these are typically $\alpha$-helical structures. Transmembrane proteins can be predicted by applying a transmembrane hidden Markov model (tmHMM) that incorporates the hydrophobicity, charge bias, helix lengths and grammatical constraints of known transmembrane proteins into one model [35]. In addition, a comprehensive list of A. niger sugar transporters can be made by applying hidden Markov models for both the major facilitator superfamily (HMMMFS), and sugar porters (HMMSP). As the largest subfamily of the MFS, the sugar porter (SP) family currently has the most identified members [36], and as such provides a good basis for constructing a specific profile HMM from multiple alignments of extensively characterized members. The experimental steps and complementary bioinformatics pipeline to identify and validate $A$. niger glucose transporters are depicted in figure 1. Initial in silico analysis of the theoretical $A$. niger proteome with the precomputed HMMMFS and HMMSP, obtained from the Pfam database [37], showed that more than 250 of the proteins predicted in A. niger ATCC1015 have a conserved domain architecture related to sugar transport (Table 1). Very similar results were obtained for the A. niger CBS 513.88 strain (not shown). This high abundance of sugar transporters makes A. niger a versatile host for the bioconversion of lignocellulosic biomass to products of interest, especially also in comparison to S. cerevisiae, which cannot metabolise as many sugars and consequently has fewer sugar transporters [11]. Although the HMMSP captures the conserved domain architecture of potential sugar porters, it cannot discriminate between different sugar substrates, and was thus considered to be too broad for the purpose of this study. Therefore, a list of 42 biochemically characterized glucose transporters from 10 different organisms was obtained from the UniProt database [38], and a profile HMM specific for glucose transporters (HMMgluT) was built from these sequences (see alignment in Additional file 1). The functions of proteins are often more conserved in their tertiary structure than in their primary amino acid sequence, as residues that are not crucial for the function of the protein will be subject to evolutionary changes over time. 


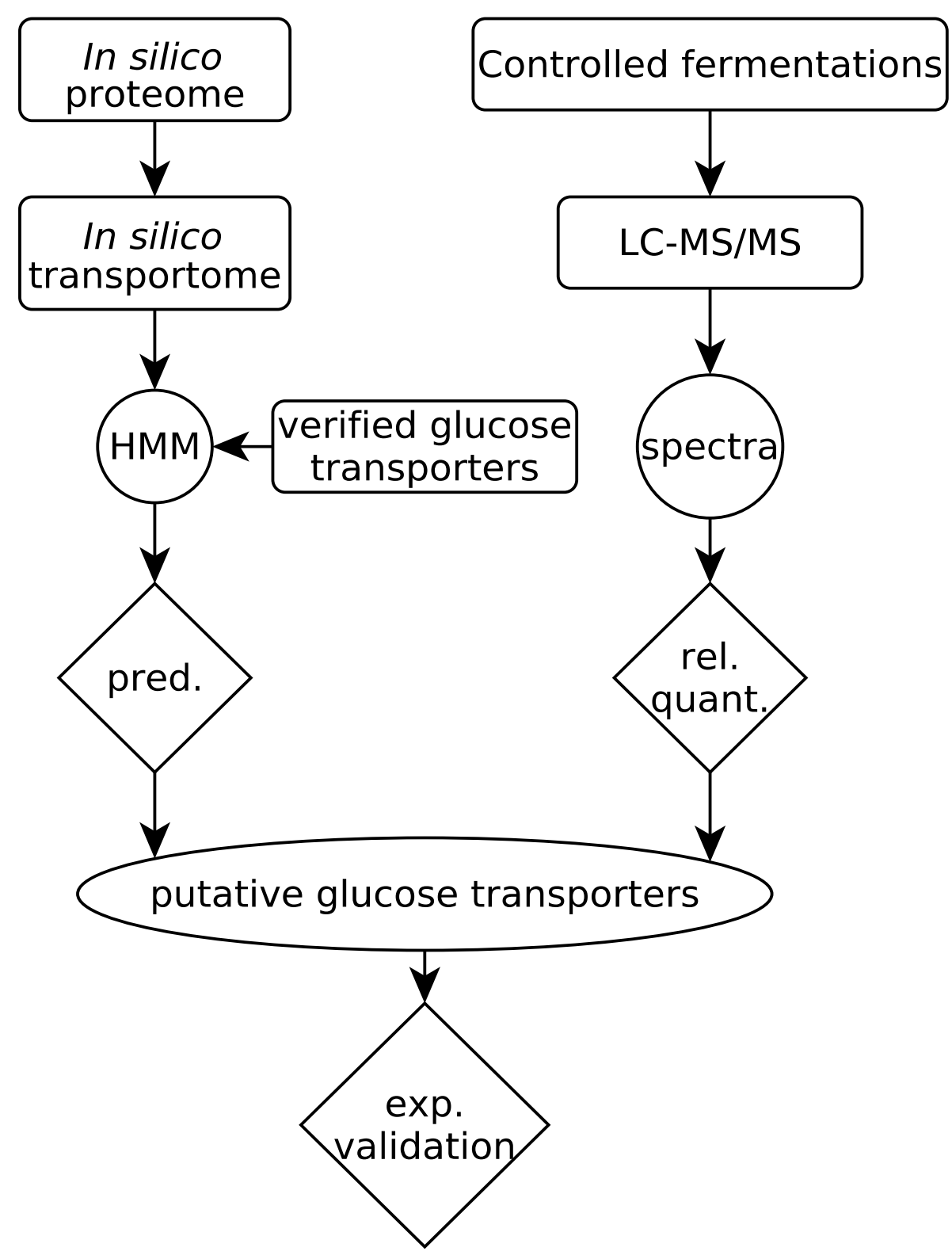

Figure 1: Bioinformatic and proteomic profiling of the $A$. niger transportome. The flow chart outlines the complementary approach taken to identify putative glucose transporters. Experimental validation of the transporters identified was carried out in S. cerevisiae 
The typical structure of known glucose transporters comprises 12 transmembrane helix domains divided into 2 groups of 6 helixes [39, 40], and the HMMgluT was computed from a structure-based multiple sequence alignment incorporating transmembrane helix predictions, rather than using an alignment algorithm based on the primary amino acid sequence alone. Appropriate threshold values, above which a hit with the HMMgluT has to score in order to be considered a true positive, can be calculated by evaluating the properties of the HMMgluT. Receiver operating characteristic (ROC) curves are instrumental in assigning the best threshold values, since they display the tradeoff between sensitivity and specificity. In order to obtain ROC curves, the HMMgluT was first validated using a 10 x 3-fold cross-validation approach (Figure 2). Threshold values with the best trade-off between the true and false positive rates of the prediction were determined from the resulting ROC curve. In this study, two thresholds were used. The first is dmin, which is the point on the curve that has the minimal distance to [0 1] in the 2-dimensional $\mathrm{x}-\mathrm{y}$ plane. Another way to determine the best trade-off point is by calculating the point in which the Matthews correlation coefficient is maximal (MCCmax), as the prediction is regarded as better the closer the MCC value is to 1 , whereas a value of 0 is regarded as no better than a random guess. To compare the performance of this approach to a more traditional approach, in which Blast is used to identify homologous proteins, each of the 42 verified glucose transporters was used as query in a separate Blast search against the same dataset used to evaluate the HMMgluT. The resulting ROC curves for each of the methods is depicted in Additional file 2 and shows that, for the datasets used, an approach using HMMs outperforms Blast in discriminating glucose transporters from other sugar transporters. By using the previously calculated thresholds as lower limits for the HMMgluT score, the list of putative glucose transporters was effectively narrowed down to 19 sugar porters when the dmin threshold was applied, and to only 5 sugar porters, including MstA, when the MCCmax threshold was applied (Table 1). Note that MstA was truly 'discovered' by the HMMgluT, since it was not a priori included in the training set. The average sensitivity, specificity, accuracy and inferred HMMgluT scoring values at the two threshold points, dmin and MCCmax, are noted in Table 2. As expected, the specificity at the MCCmax threshold is very high, but it comes at the cost of having a lower sensitivity. The dmin threshold on the other hand is less strict, thus allowing for a higher false-positive rate in order to not neglect any true positives. 


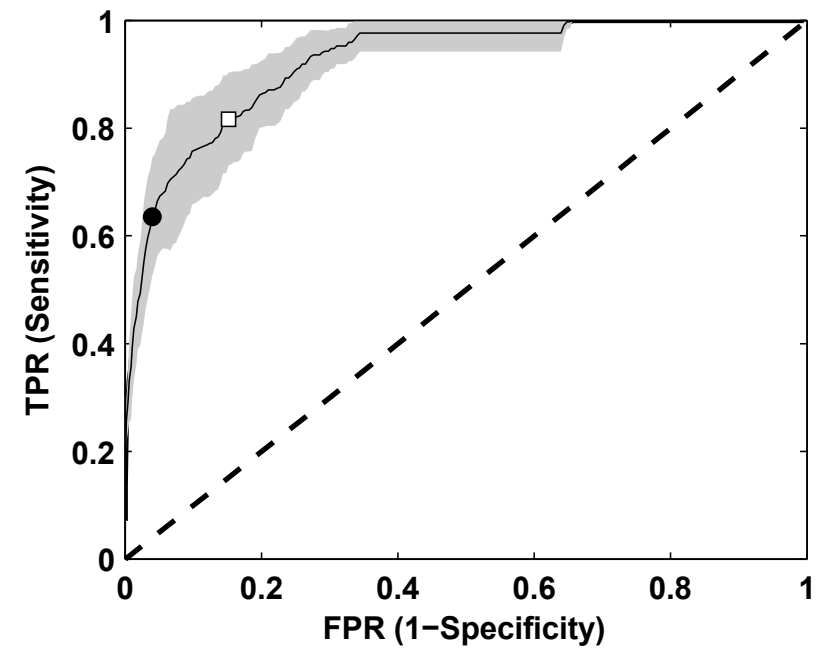

Figure 2: Receiver operating characteristic (ROC) curve for the HMMgluT transporter model. Plotted is the mean of 30 runs of a $10 \times 3$-fold cross-validation. The confidence interval is shown in grey. Calculated inclusion thresholds, $d_{\text {min }}$ (white square) and $M C C_{\max }$ (black circle), are indicated.

Table 1 MFS domain proteins in A. niger and S. cerevisiae

\begin{tabular}{lccccccc}
\hline & $\mathbf{N}_{\text {tot }}$ & $\mathbf{N}_{\text {tmHMM }}$ & $\mathbf{N}_{\text {MFS }}$ & $\mathbf{N}_{\text {SP }}$ & $\mathbf{N}_{\text {glut }}$ & $\mathbf{N}_{\text {gluT }}\left(\boldsymbol{d}_{\min }\right)$ & $\begin{array}{c}\mathbf{N}_{\text {gluT }} \\
\left(\mathbf{M C C}_{\max }\right)\end{array}$ \\
\hline A. niger ATCC1015 & 11910 & 2561 & 469 & 256 & 252 & 19 & 5 \\
S. cerevisiae CEN.PK & 5439 & 1022 & 73 & 43 & 45 & 15 & 12 \\
\hline
\end{tabular}

$\overline{N_{t o t}}$ total number of predicted proteins, $N_{t m H M M}$ number of proteins containing at least one transmembrane helix domain, $N_{M F S}$ number of proteins containing an MFS domain, $N_{S P}$ number of proteins containing an

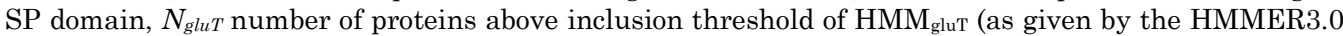
tool), $N_{g l u T}\left(d_{\text {min }}\right)$ number of proteins above the inferred threshold score at $d_{\min }, N_{g l u T}\left(\mathrm{MCC}_{\max }\right)$ number of proteins above the inferred threshold score at $\mathrm{MCC}_{\max }$

Table 2 Average sensitivity, specificity, accuracy and inferred $\mathrm{HMM}_{\text {gluT }}$ threshold values for the $\mathrm{HMM}_{\mathrm{gluT}}$ at $d_{\min }$ and $M C C_{\max }$

\begin{tabular}{lcccc}
\hline Sensitivity (\%) & $\begin{array}{c}\text { Specificity } \\
(\%)\end{array}$ & $\begin{array}{c}\text { Accuracy } \\
(\%)\end{array}$ & $\begin{array}{c}\text { HMM }_{\text {glut }} \\
\text { score }\end{array}$ \\
\hline$d_{\min }$ & 81.67 & 84.79 & 84.61 & 379.41 \\
$\mathrm{MCC}_{\max }$ & 63.57 & 96.00 & 94.12 & 531.03 \\
\hline
\end{tabular}




\section{The $A$. niger plasmalemma proteome in response to different glucose concentrations at low $\mathbf{p H}$}

A way to verify both the $\mathrm{HMM}_{\mathrm{glu}}$ and the predicted glucose transporter candidates is by using an experimental setup, in which the abundance of transmembrane proteins in relevant conditions can be compared with their HMMglut scores. In this complementary work, the focus was put on the study of the A. niger transportome in the presence of different concentrations of glucose at a $\mathrm{pH}$ which is both physiologically and biotechnologically relevant for this fungus. Mycelium of A. niger N400 was pregrown for $18 \mathrm{~h}$ in minimal medium with $100 \mathrm{mM}$ sorbitol as sole carbon source and subsequently transferred in equal amounts to controlled fermenters containing minimal medium with the following carbon source compositions: $100 \mathrm{mM}$ sorbitol (reference condition), $100 \mathrm{mM}$ sorbitol plus $1 \mathrm{mM}$ glucose (low-glucose condition) and $100 \mathrm{mM}$ sorbitol plus $60 \mathrm{mM}$ glucose (high-glucose condition). The initial $\mathrm{pH}$ of these cultures was set at $\mathrm{pH} 4.0$, which corresponded with the final $\mathrm{pH}$ measured at the pre-growth stage. Immediately after inoculation the $\mathrm{pH}$ of the fermenter cultures started to

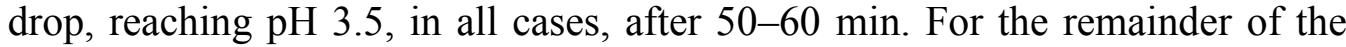
experiment this was kept constant at $\mathrm{pH} 3.5$. High-resolution analysis of the sugar content in the culture medium sampled $2 \mathrm{~h}$ after inoculation showed that there was sorbitol consumption in the reference condition; sorbitol and glucose consumption in the low-glucose condition; and glucose but no sorbitol consumption in the high-glucose condition. This result confirmed, as reported [41], that the organism strongly favors consumption of good carbon sources like glucose, over poorer carbon sources, such as sorbitol, even when the latter is also present at a high concentration $(100 \mathrm{mM})$. The 2-h time-point was selected for membrane-associated protein analysis in all three conditions. Isolation of fractions enriched for cell membranes was performed using a protocol similar to the one developed by Oliveira and co-workers [32]. This protocol involves the previously described workflow: cell disruption, crude organelle separation, and subsequent enrichment [42]. After several differential centrifugation steps, a pellet containing crude low-density organelles (P3) was obtained and subjected to density gradient centrifugation, yielding a set of five fractions (P3A-P3E). The PM marker vanadate-sensitive $\mathrm{H}_{+} / \mathrm{ATPase}$ and the mitochondrial membrane cytochrome c oxidase activities were then measured in the initial cell-free extract, the P3 pellet and the P3A to P3E fractions derived from it. Compared to the cellfree extract, the P3 pellet was shown to be 2.4-3.2 times enriched in plasma membranes. No further enhanced PM enrichment was found in the P3A to P3E fractions; however, cytochrome c oxidase activity was higher in the P3A to P3E fractions when compared to P3. Since mitochondrial membranes were not the 
main focus of this research, the P3 pellets, considered to be more optimal for the analysis of plasmalemma proteins, were further processed and subjected to shotgun proteomics analysis (detailed information regarding subcellular fractionation, marker enzyme assays and sample preparation for LC-MS/MS can be found in the "Methods"). The LC-MS/MS spectra obtained were processed as described in the "Methods". In total, 833 proteins were identified, of which 432 were present in all three conditions, 72 proteins were found only in the reference condition, 34 only in the low-glucose condition, and 106 proteins were found exclusively in the high-glucose condition. Of the proteins identified, almost 30 $\%$ had one or more predicted ${ }_{\mathrm{tm}} \mathrm{HMM}$ domains, indicative of integral membrane proteins (Additional file 3). The relative abundances of the proteins containing at least one ${ }_{\mathrm{tm}} \mathrm{HMM}$ domain, which add up to $15.28,17.50$ and $15.45 \%$ of the total protein isolated in the reference, low- and high glucose conditions, respectively, can be found in Additional file 4. Proteins associated with the mitochondrial membrane were most abundant, followed by the proteins associated with the plasma membrane. However, the plasma membrane-associated fraction consists of a higher number of different proteins than the mitochondrial membraneassociated fraction, with 86 and 35 identified proteins, respectively. Proteins associated with the membrane-bound endoplasmic reticulum (ER) constitute the third most abundant group of the list, with a total of 48 different proteins identified. Finally, proteins linked to other membrane-associated organelles, comprising the endomembrane system and membrane-bound organelles, such as the Golgi apparatus, vacuoles and lysosomes, account for $<1 \%$ of the total protein isolated (Additional file 5). In all three conditions, approximately onethird of the proteins that have one or more ${ }_{\mathrm{m}} \mathrm{HMM}$ domains are annotated as proteins with a transporter function. This fraction, denoted as the A. niger transportome, accounted for 3.9, 4.4 and $4.1 \%$ of the total protein isolated in the reference, low- and high-glucose conditions, respectively. Mitochondrial carrier proteins are prevalent in all three conditions, followed by ATPases. Proteins with the MFS architecture, which are the main interest in this study, comprise the third most abundant group of the isolated A. niger transportome. Proteins (putatively) involved in the secretory pathway and amino acid transport were found in relative abundances of $<0.5 \%$ of the total protein isolated. Other transporters, comprising (putative) oligopeptide transporters, formate/nitrite transporters, ammonium transporters, iron permeases, $\mathrm{Na}+/$ solute symporters, $\mathrm{ABC}$ transporters, inorganic phosphate transporters, nucleotide-sugar transporters, major intrinsic proteins and the translocation protein Sec62, accounted for $\leq 0.01 \%$ of the total protein isolated, respectively. 


\section{Performance of the HMMgluT for the identification of candidate glucose transporters}

The A. niger transportome was further analyzed for the presence of glucose transporters. Table 3 summarizes the results of the bioinformatics analysis, which was performed in the same way as previously for the entire A. niger in silico proteome, i.e. the data were first queried with the hidden Markov models specific for proteins containing a MFS or SP domain. As already observed for the $A$. niger in silico proteome, a search with the HMMsp lowered the number of proteins that scored above the inclusion threshold in comparison to the search performed with the less specific HMMMrs. Querying the transportome with the newly developed HMMgluT, using the previously calculated thresholds, lowered the number of putative, albeit highly probable, glucose transporters to 4, 3, and 2 promising hits in the reference, low- and high-glucose conditions, respectively. The relative abundances of the identified MFS proteins that scored above the default inclusion threshold of HMMgluT, HMMsp and HMMMFs, ordered by their HMMglut scores, can be found in Table 4. High HMM scores corresponded to MFS porters that have been related to glucose uptake in previous studies, or novel MFS porters that, in the present study, showed abundance patterns that point towards a possible role as glucose transporter. In figure $\mathbf{3 A}$, the proteins found in the three conditions are highlighted on the HMM curves for the glucose and general sugar transporter model depicted. The color-coding corresponds to their relative abundances in the three conditions (see "Methods" for details). In figure $\mathbf{3 A}$, the proteins found in the three conditions are highlighted on the HMM curves for the glucose and general sugar transporter model depicted. The color-coding corresponds to their relative abundances in the three conditions (see "Methods" for details). The HMM curves as such show the scores of all proteins in the theoretical A. niger ATCC1015 proteome that hit above the default inclusion threshold of both HMMgluT and HMMsp. The majority of proteins that were more abundant in the low-glucose condition relative to both the reference and highglucose condition cluster closer to the $\mathrm{d}_{\min }$ and $\mathrm{MCC}_{\max }$ thresholds, whereas the proteins that were more abundant in the high-glucose condition scored overall lower on the HMMglut. This indicates that the model is better at detecting highaffinity glucose transporters, which might be due to the sequences that were selected to build the HMMgluT (Additional file 1).

Table 3 Number of MFS porter proteins found in the three growth conditions

\begin{tabular}{|c|c|c|c|c|c|}
\hline 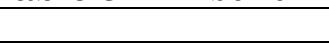 & $\mathbf{N}_{\mathrm{MFS}}$ & $\mathbf{N}_{\mathrm{SP}}$ & $\mathbf{N}_{\text {glut }}$ & $\mathbf{N}_{\text {glut }}\left(d_{\text {min }}\right)$ & $\mathbf{N}_{\text {glut }}\left(M^{\prime} C_{\text {max }}\right)$ \\
\hline Sorbitol & 14 & 13 & 13 & 4 & 4 \\
\hline Sorbitol + $1 \mathrm{mM}$ glucose & 9 & 6 & 7 & 3 & 3 \\
\hline Sorbitol + $60 \mathrm{mM}$ glucose & 15 & 9 & 10 & 2 & 2 \\
\hline
\end{tabular}



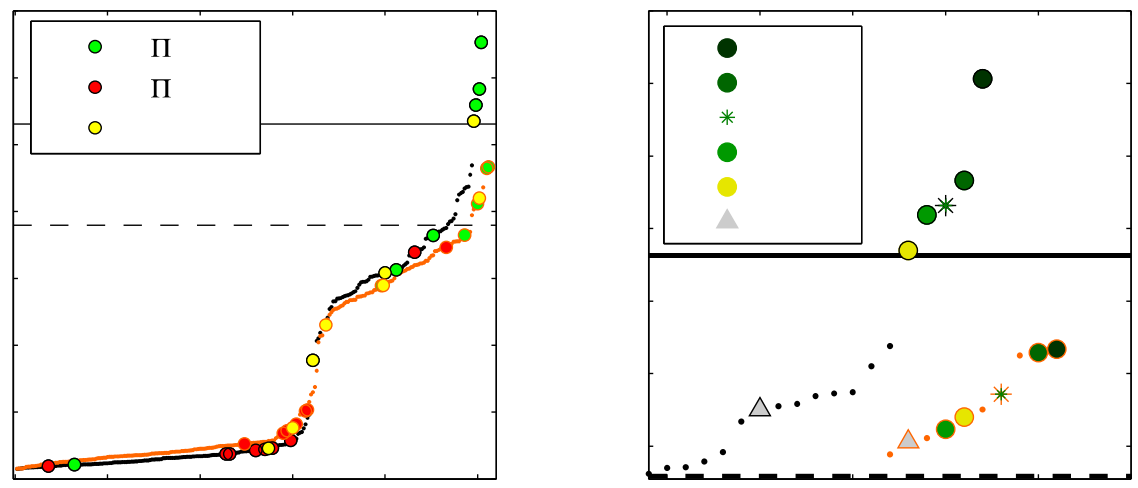

Figure 3 HMMSP and HMMgluT scores of MFS porter proteins. A. HMM scores for the theoretical ATCC1015 proteome, with HMMgluT = black dotted line and HMMSP = orange dotted line. Horizontal lines indicate the HMMgluT thresholds dmin (dashed) and MCCmax (solid). MFS porter proteins found in the three conditions applied are indicated with filled circles and colour-coded according to their relative abundance patterns. B. Close-up of the proteins that scored above the dmin (dashed line) and MCCmax (solid line) thresholds. The galacturonic acid transporter GatA (indicated with a grey triangle and not found in the conditions applied) is added as a reference. With HMMgluT, the Mst transporters are well separated from GatA, whereas the more general HMMSP does not discriminate the putative glucose transporters from the galacturonic acid transporter

A close-up of the top scoring proteins is depicted in figure 3A. The highest score was obtained with protein ID 1121621, which is the putative low-affinity glucose transporter MstC. Studies on its transcriptional regulation during the exponential growth phase in batch fermentation and in chemostat cultures, carried out at different dilution rates, have been performed by Jørgensen et al. [9]. The mstC transcripts were only detected during the batch fermentation phase, indicating that $m s t C$ expression is associated with higher glucose concentrations or with specific growth rates. In the present work, the protein abundance of the MstC protein was found to be similar in all the three conditions studied. This result, together with the previous findings of Jørgensen et al., suggests that the presence of MstC is independent of the glucose concentration. The second best hit, with protein ID 1142882, and the fourth best hit, with protein ID 1143598, are yetuncharacterised transport proteins, henceforth denoted as MstG and $\mathrm{MstH}$, respectively. Their high $\mathrm{HMM}_{\mathrm{glu}}$ scores and overall abundance pattern in the three experimental conditions strongly indicate a possible role for them as glucose transporters. MstG and MstH were found with relatively high abundance levels only in the two non-carbon catabolite-repressing conditions studied, being 
higher in abundance when low glucose concentrations were present. This type of abundance pattern fits that of high-affinity glucose transporters described in $S$. cerevisiae and Aspergillus species [14, 43, 44], that are preferably expressed in the presence of low concentrations of glucose, poor carbon (de-repressing) sources and in starvation conditions. Another protein with protein ID 1125134, scoring in between MstG and MstH in the HMMgluT was not observed in the three studied conditions. It is denoted as MstE (An03g02190) in the UniProt database, and has been found to be expressed in germinating spores [45]. The fifth highest scoring hit, with protein ID 1143191, is MstA. The mstA coding gene is transcriptionally controlled by the carbon catabolite repressor CreA and the environmental $\mathrm{pH}$ regulator $\mathrm{PacC}$. Its transcript levels were found to be higher during carbon starvation at $\mathrm{pH} 6.0$ [14]. Van Kuyk et al. also observed low expression levels at $\mathrm{pH} 4.0$ and 8.0, and in the presence of repressing glucose concentrations at $\mathrm{pH} 6.0$ [14]. In the present study, MstA was detected in very low abundance; however, only in the de-repressing reference condition, supporting previous results that suggested a limited role of this transporter when the environmental $\mathrm{pH}$ is low. The combined results of the specific protein abundance patterns and the high $\mathrm{HMM}_{\text {gluT }}$ scores indicate that MstG and MstH could have a role as high/mid-affinity glucose transporters, therefore both transporters were selected for further characterization.

\section{Transcriptional analysis of $m s t G$ and $m s t H$ in the presence of different carbon sources}

In order to obtain additional insights in the regulation and possible biological role of MstG and $\mathrm{MstH}$, transcriptional levels were analyzed in mycelium samples from cultures containing: minimal medium with $100 \mathrm{mM}$ sorbitol (used as a reference condition) and minimal medium plus glucose, fructose or mannose at high $(55 \mathrm{mM})$ or low $(5 \mathrm{mM})$ concentration. Samples taken $2 \mathrm{~h}$ after mycelium transfer were processed and RTqPCR analysis was performed. Both genes were found to be expressed in all the studied conditions, but their expression levels were clearly influenced by the different culture conditions (Figure 4). The relative transcript levels of both genes in the reference, low-glucose and high glucose conditions showed a pattern comparable to what was observed at proteomic level; $m s t G$ and $m s t H$ were upregulated in the presence of a low glucose concentration and downregulated when the glucose concentration was high. However, $m s t G$ and $m s t H$ expression patterns showed to be divergent in the presence of high and low concentrations of fructose and mannose. With these carbon sources $m s t G$ expression levels were similar to those observed with a low concentration of glucose. In the case of $m s t H$, as observed in the presence of 
glucose $55 \mathrm{mM}$, high concentrations of fructose $(55 \mathrm{mM})$ and mannose $(55 \mathrm{mM})$ also led to a decrease of its transcript levels when compared to the reference condition. While these results do not provide conclusive evidence that MstG is indeed a high-affinity glucose transporter they are not in disagreement with its possible role either. In the case of $m s t H$, only the glucose $5 \mathrm{mM}$ condition enhanced its expression, suggesting that $\mathrm{MstH}$ could have a specific role in $A$. niger when low concentrations of the monosaccharide are available.

Table 4 Relative abundance and HMM scores of MFS porter proteins detected in the three growth conditions

\begin{tabular}{|c|c|c|c|c|c|c|c|}
\hline \multirow[t]{2}{*}{ protID } & \multirow[t]{2}{*}{$\mathrm{HMM}_{\text {glut }}$} & \multirow[t]{2}{*}{ HMM $_{S P}$} & \multirow[t]{2}{*}{$\mathrm{HMM}_{\mathrm{MFS}}$} & \multicolumn{3}{|c|}{ Relative abundance $\pm \mathrm{sd}(\%) \times 100$} & \multirow[t]{2}{*}{ Remark } \\
\hline & & & & Sorbitol & $\begin{array}{l}\text { Sorb + } 1 \mathrm{mM} \\
\text { glucose }\end{array}$ & $\begin{array}{l}\text { Sorb }+60 \mathrm{mM} \\
\text { glucose }\end{array}$ & \\
\hline 1121621 & 653.1 & 467.0 & 78.5 & $9.08 \pm 1.02$ & $11.89 \pm 4.79$ & $9.47 \pm 3.50$ & $\begin{array}{l}\text { MstC } \\
\text { (Q8J0U9) }\end{array}$ \\
\hline 1142882 & 583.1 & 464.5 & 74.8 & $2.24 \pm 0.48$ & $4.47 \pm 0.20$ & n.d. & MstG \\
\hline 1143598 & 559.3 & 411.7 & 86.1 & $6.38 \pm 1.83$ & $17.54 \pm 2.39$ & n.d & MstH \\
\hline 1143191 & 535.0 & 420.0 & 75.7 & $8.51 \mathrm{e}^{-3} \pm 1.14 \mathrm{e}^{-3}$ & n.d. & n.d. & $\begin{array}{l}\text { MstA } \\
\text { (Q8J0V1) }\end{array}$ \\
\hline 1101809 & 364.4 & 364.7 & 85.6 & $4.83 \pm 0.51$ & $2.01 \pm 0.29$ & n.d. & \\
\hline 1180703 & 339.2 & 346.3 & 97.4 & n.d. & n.d. & $1.37 \pm 0.14$ & $\begin{array}{l}\text { MstD } \\
\text { (Q8J0U8) }\end{array}$ \\
\hline 1188093 & 312.9 & 289.3 & 82.0 & $1.08 \pm 0.39$ & $0.82 \pm 0.11$ & $0.82 \pm 0.25$ & \\
\hline 1144791 & 308.1 & 289.6 & 55.9 & $6.25 \pm 1.25$ & n.d. & n.d. & \\
\hline 1189214 & 177.3 & 230.6 & 73.7 & $0.29 \pm 0.03$ & n.d. & n.d. & \\
\hline 1128338 & 57.6 & 103.6 & 72.9 & $3.70 \pm 0.32$ & $4.36 \pm 0.02$ & $6.46 \pm 0.84$ & \\
\hline 1111630 & 46.4 & 101.6 & 63.2 & n.d. & n.d. & $1.06 \pm 0.10$ & \\
\hline 1184634 & 46.0 & 76.4 & 0 & $0.30 \pm 4.12 \mathrm{e}^{-5}$ & n.d. & n.d. & \\
\hline 1122202 & 44.6 & 52.6 & 135.9 & $0.87 \pm 0.10$ & n.d. & $0.48 \pm 0.29$ & \\
\hline 1178623 & 42.9 & 68.3 & 149.3 & n.d. & n.d. & $4.71 \pm 1.07$ & \\
\hline $\begin{array}{l}\text { 1164538; } \\
1188786\end{array}$ & $\begin{array}{l}37.5 \\
37.4\end{array}$ & $\begin{array}{l}82.3 \\
72.1\end{array}$ & $\begin{array}{l}\text { 104.3; } \\
98.9\end{array}$ & $2.11 \pm 0.14$ & n.d. & $2.60 \pm 0.21$ & $\begin{array}{l}\text { Same } \\
\text { protein } \\
\text { group }\end{array}$ \\
\hline 1089440 & 21.4 & 0 & 92.5 & n.d. & $1.16 \pm 0.12$ & n.d. & \\
\hline 1118545 & 19.1 & 0 & 0 & n.d. & n.d. & $3.87 \pm 1.13$ & \\
\hline 1105147 & 0 & 0 & 54.4 & $8.93 \pm 0.03$ & $7.14 \pm 2.01$ & $11.47 \pm 1.24$ & \\
\hline 1129336 & 0 & 0 & 31.8 & $0.34 \pm 0.01$ & $0.50 \pm 0.10$ & $1.07 \pm 0.01$ & \\
\hline 1124902 & 0 & 0 & 130.7 & n.d. & n.d. & $1.37 \pm 0.44$ & \\
\hline 1165706 & 0 & 0 & 114.3 & n.d. & n.d. & $0.05 \pm 0.01$ & \\
\hline 1188840 & 0 & 0 & 99.1 & n.d. & n.d. & $3.00 \pm 1.30$ & \\
\hline 1146101 & 0 & 0 & 65.3 & n.d. & n.d. & $1.21 \pm 0.91$ & \\
\hline
\end{tabular}



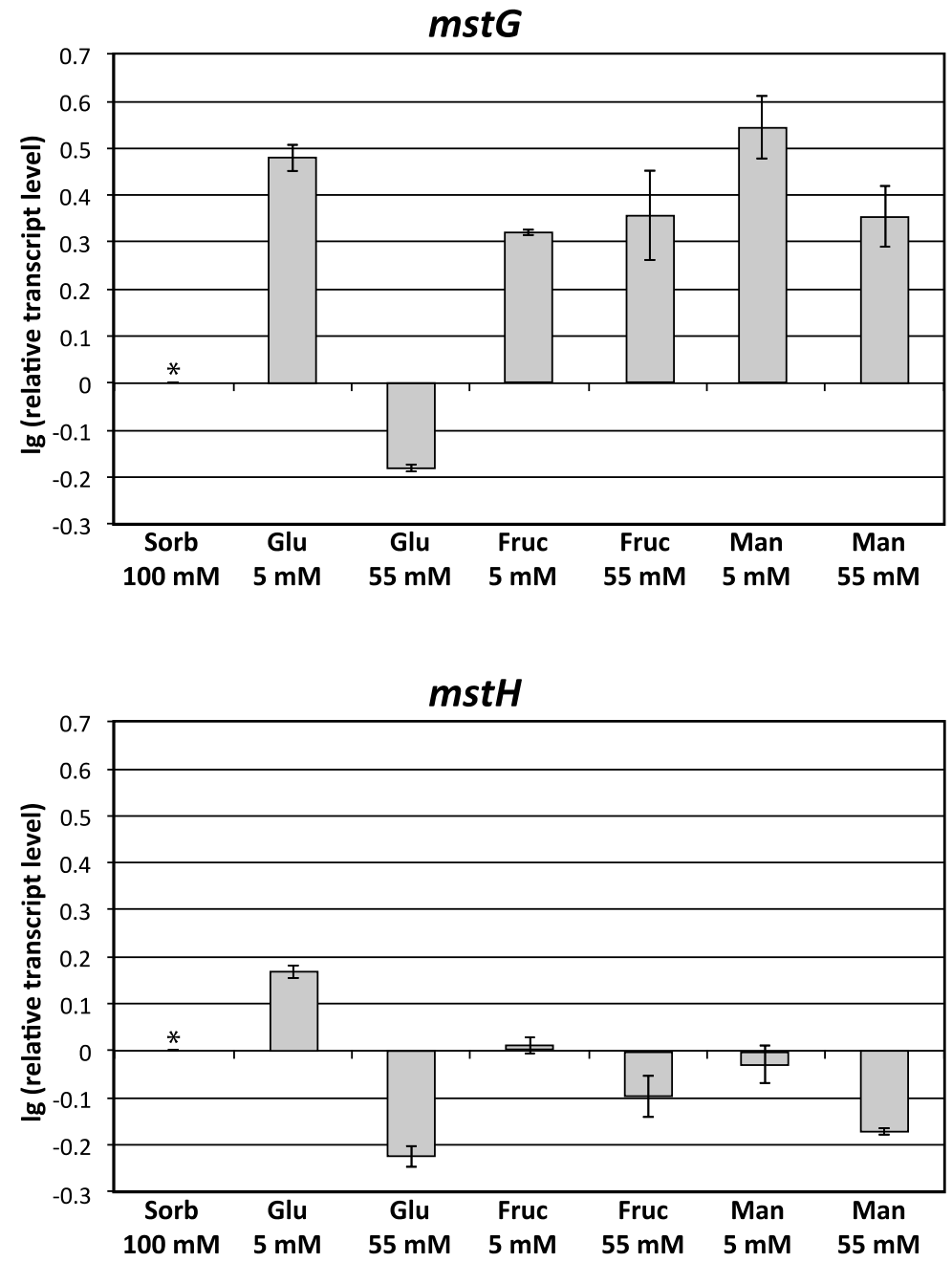

Figure 4 Transcriptional analysis of $\mathrm{mstG}$ and $\mathrm{mstH}$. Mycelium of the A. niger strain N400 was precultured on minimal medium with sorbitol $100 \mathrm{mM}$ and thereafter transferred to minimal medium with the following carbon sources: sorbitol $100 \mathrm{mM}$, glucose $5 \mathrm{mM}$, glucose $55 \mathrm{mM}$, fructose $5 \mathrm{mM}$, fructose $55 \mathrm{mM}$, mannose $5 \mathrm{mM}$ and mannose $55 \mathrm{mM}$. Samples from the seven culture conditions were taken $2 \mathrm{~h}$ after mycelium transfer, and expression analyses of $\mathrm{mstG}$ and $\mathrm{mstH}$ were performed by qPCR using a histone-like gene (gene ID 207921) transcript for normalization. Results are given as relative transcript rations in logarithmic scale (lg). The values provided in the figures are means of two biological replicates. Transcript levels always refer to the reference sample (sorbitol $100 \mathrm{mM}$ ), indicated with an asterisk 


\section{Functional validation of $\boldsymbol{A}$. niger sugar transporters MstG and MstH}

As discussed above, the high HMMgluT scores, the protein abundance patterns and transcriptional analyzes of the MFS porters MstG and MstH point towards a role in glucose uptake. In order to test their functionality, the engineered S. cerevisiae strain EBY.VW.4000 [12], a glucose transporter null mutant unable to grow on glucose, mannose, galactose or fructose as carbon source, was chosen as host for functional complementation analysis. The yeast strain was transformed with the $2 \mu$ expression plasmid p426HXT7-6His-mstG or p426HXT7-6His-mstH, containing the respective cDNA under control of the constitutive promoter $\mathrm{HXT}_{\mathrm{p}}$ and the terminator $\mathrm{CYCl}_{\mathrm{t}}$. Single colony transformants were isolated from minimal medium agar plates containing $2 \%$ maltose and the ability of both genes to restore growth of the EBY.VW.4000 transformant strain in the presence of different monosaccharides was studied. Tenfold serial dilutions of exponentially growing cells from at least two different transformants expressing each gene were spotted on different minimal medium plates supplemented with $1 \%(\mathrm{w} / \mathrm{v})$ of the following carbon sources: glucose, galactose, fructose, mannose, sucrose and maltose (Figure 5). The $m s t G$ transformants were able to grow on glucose, galactose, mannose and sucrose as single carbon sources, whereas $m s t H$ transformants grew on glucose, sucrose, mannose, galactose and, in contrast to $m s t G$ strains also on fructose. MstH transformants also showed better growth on mannose and sucrose, but poorer growth on galactose. Regarding the sucrose transport by both transporters, since the EBY.VW4000 strain encodes an extracellular invertase [18], it is unknown if the transported substrate was sucrose or glucose in the case of MstG, and sucrose, glucose or fructose in the case of $\mathrm{MstH}$. These results indicated that $\mathrm{MstG}$ and $\mathrm{MstH}$ are functional sugar transporters with the ability to transport a variety of substrates. In order to have better insights about the affinity for glucose of both transporters, the growth rates of the transformants expressing MstG and MstH were studied in the presence of different glucose concentrations. Additional file 6 shows growth curves and glucose consumption figures of the transformant strains grown on minimal medium with glucose $2.5,10$ and $50 \mathrm{mM}$. Both transporters showed to be functional in all glucose concentrations. However, when comparing individual growth curves and glucose uptake, some differences could be observed. The growth rate of the MstG transformant during the exponential growth phase was comparable in the three different conditions, while in case of the MstH transformant, at the highest glucose concentration the growth rate was reduced. 


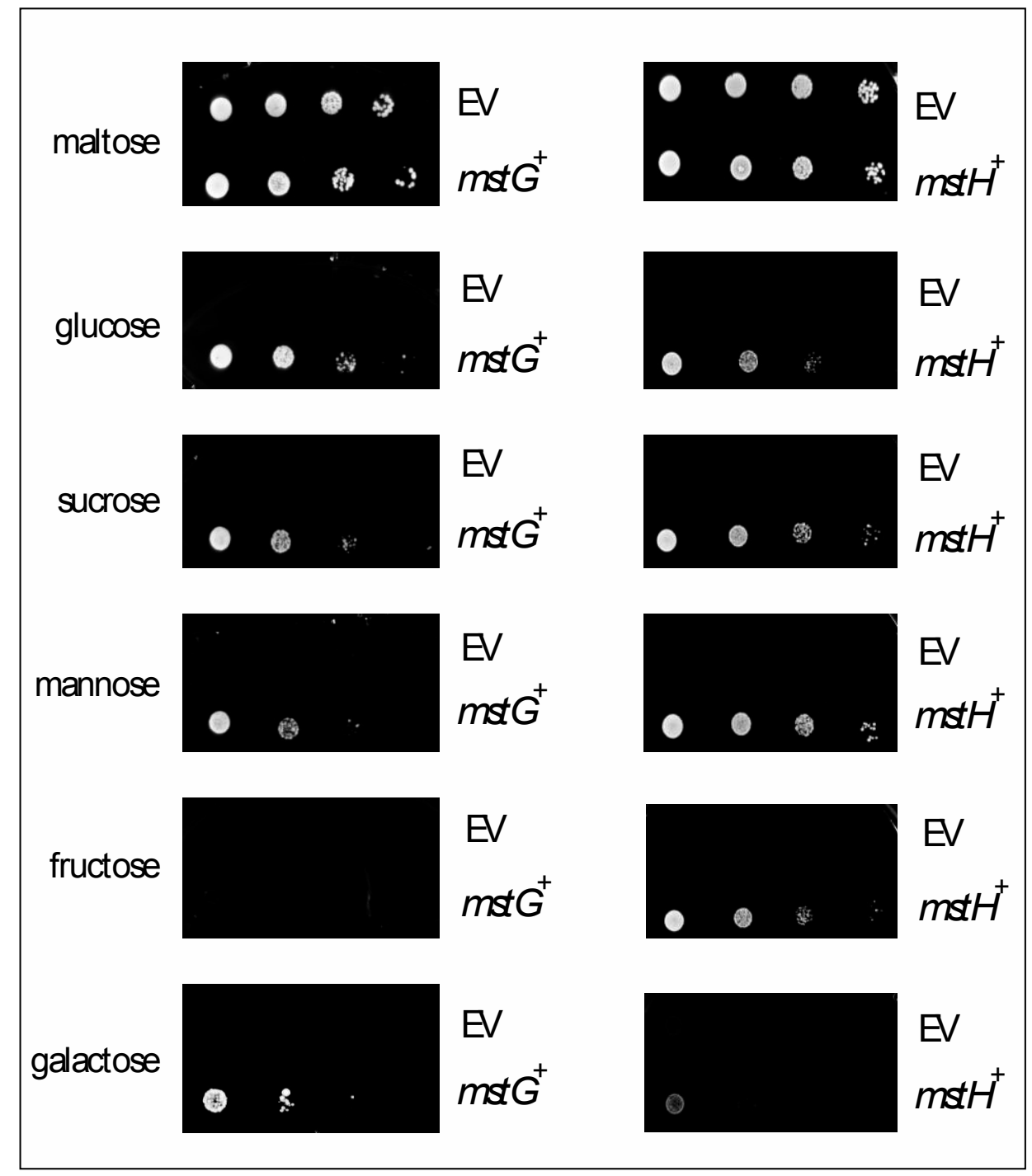

Figure 5 MstG and MstH functional analysis. Growth of strain EBY.VW4000 containing the $m s t G$ gene (mst $G+)$, mstH gene $(m s t H+)$, or harboring the empty expression vector $\mathrm{p} 426 \mathrm{HXT} 7-6 \mathrm{His}$ (EV) in minimal medium agar plates containing the following sugars at a final concentration of $1 \%(w / v)$ : maltose, glucose, sucrose, mannose, fructose and galactose. Agar plates were incubated at $30{ }^{\circ} \mathrm{C}$ for $96 \mathrm{~h}$. All transformants expressing each gene showed the same growth pattern; therefore, the figure depicts only one transformant per transporter as representative 
Accordingly, the glucose consumption rate of the MstG transformant in the 50 $\mathrm{mM}$ condition was much faster, being able to deplete the monosaccharide after around $40 \mathrm{~h}$ of growth, whereas in the same timespan the MstH transformant was only able to consume a $60 \%$ of the total amount suggesting that MstG and MstH are glucose transporters with different affinities for the sugar. To determine MstG and $\mathrm{MstH}$ transport characteristics, $\left({ }^{14} \mathrm{C}\right)$ glucose transport assays were performed with the transformant strains as described [46]. Initial glucose uptake rates at various substrate concentrations were fitted to the Michaelis-Menten model with noncompetitive substrate inhibition and used to estimate the appropriate kinetic parameters as described [47]. MstG was confirmed to transport glucose with apparent $\mathrm{K}_{\mathrm{m}}$ values of $0.5 \pm 0.04 \mathrm{mM}$, and $\mathrm{V}_{\max }$ of $5.8 \pm$ $0.04 \mathrm{nmol} \mathrm{min}_{-1} \mathrm{mg} \mathrm{DW}_{-1}$ (Figure 6a). Its affinity for glucose was lower than the one reported for $A$. niger MstA with a $\mathrm{K}_{\mathrm{m}}$ value of $0.025 \pm 0.01 \mathrm{mM}$. However, MstG can still be classified as a high-affinity glucose transporter, since its $\mathrm{K}_{\mathrm{m}}$ value is lower than the one reported for the $S$. cerevisiae high-affinity hexose transporter HXT6 $\left(\mathrm{K}_{\mathrm{m}}\right.$ value $\left.1.4 \pm 0.1 \mathrm{mM}\right)$ [47]. Initially MstH kinetics were determined using the same range of substrate concentrations as for MstG. The results obtained suggested that MstH had a much higher affinity for glucose than MstG. Therefore, the assay was optimised and repeated using a micro-molar range of glucose concentrations. MstH was confirmed to transport glucose with apparent $\mathrm{K}_{\mathrm{m}}$ values of $0.06 \pm 0.005 \mathrm{mM}$ and a $\mathrm{V}_{\max }$ of $1.3 \pm 0.2 \mathrm{nmol}$ min-1 $\mathrm{mg}$ $\mathrm{DW}_{-1}$ (Figure 6b), similar to what has been reported for $A$. niger MstA. Since MstG and MstH were able to restore growth of the hexose non-transporting EBY.VW4000 on other monosaccharides as well, their substrate specificity was evaluated by determining glucose relative transport levels in the presence of a tenfold excess of various competing sugars (Figure 6c). Both transporters clearly showed higher specificities for glucose. However, besides glucose, galactose, fructose and xylose were able to inhibit MstG glucose transport by 39, 29 and 25 $\%$, respectively; whereas, in the $\mathrm{MstH}$ transformant mannose and fructose reduced the uptake of labeled glucose by 70 and $50 \%$, respectively. A. niger MstA also showed a preference for glucose, while being able to transport other monosaccharides as well [14]. Similarly, substrate specificity of Aspergillus nidulans hexose transporters $\mathrm{HxtB}$ and $\mathrm{HxtC}$ have been assayed [20], and a reduction of glucose uptake of $70-80 \%$ was observed for galactose, fructose and mannose. In accordance to the functioning of many glucose transporters from other filamentous fungi, the function of $\mathrm{MstG}$ and $\mathrm{MstH}$ was found to be energy dependent via proton symport. This was confirmed by measurements of glucose initial uptake rate in the presence of CCCP that resulted in a $60 \pm 8 \%(n=3)$ and 
$64 \pm 6 \%(n=3)$ reduction of labelled glucose uptake by MstG and MstH, respectively.

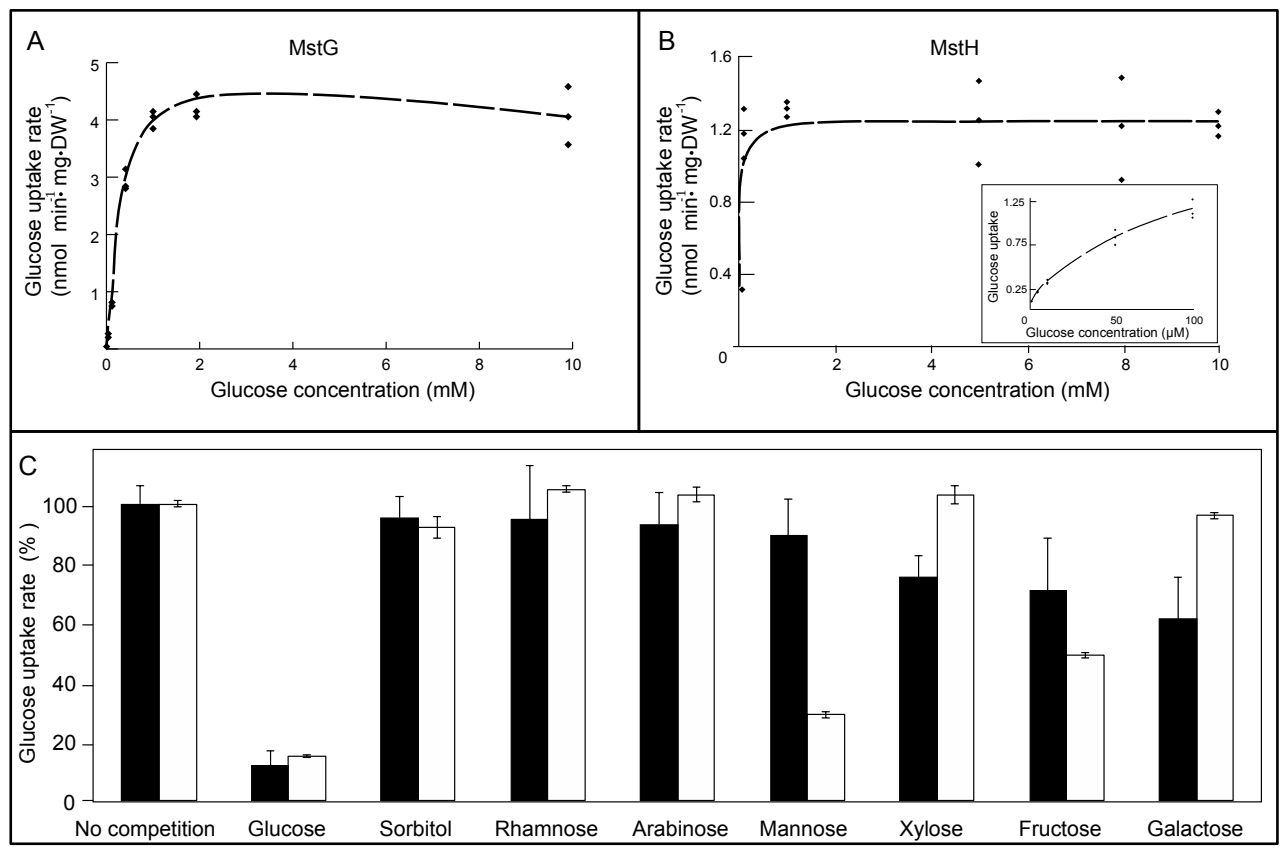

Figure 6 Uptake of ${ }^{14} \mathrm{C}$-labelled glucose by the yeast hexose transporter null mutant EBY.VW4000 expressing $m s t G$ or $m s t H$ as a function of glucose concentration. $\mathbf{a}, \mathbf{b}$ Diamonds represent measured uptake rates of ${ }^{14} \mathrm{C}$ labelled glucose $(n=3)$ as a result of expression of $m s t G$ (a) or $m s t H$ (b). Black lines represent least-squares-fitted Michaelis-Menten model ( $m s t G ; K_{m}=0.5 \mathrm{mM}$; Vmax $=5.8 \mathrm{nmol}$ min-1 mg DW-1; $m s t H: K_{m}=0.06 \mathrm{mM}$, and a $\left.V_{\max }=1.3 \mathrm{nmol} \min -1 \mathrm{mg} \mathrm{DW}-1\right)$. Insert results for $m s t H$ using micro-molar substrate concentrations. c Uptake rate of $1 \mathrm{mM}{ }^{14} \mathrm{C}$ labelled glucose by EBY.VW4000 expressing $m s t G$ (filled boxes) or $m s t H$ (open boxes) in the presence of a tenfold excess of competing sugars 


\section{Conclusions}

In this study, two complementary approaches were used to both increase the understanding of the A. niger membrane-associated proteome, and to identify novel glucose transporters. In a purely in silico approach, a hidden Markov model (HMMgluT) was constructed for the identification of glucose transporters. $\mathrm{HMM}_{\text {glut }}$ performed well in the identification and segmentation of functionally validated glucose transporters. In a complementary in vivo approach, defined culture conditions were applied to study the response of the $A$. niger membraneassociated proteome to different glucose concentrations. To the best of our knowledge, this is the first study on the membrane-associated proteome of the industrially relevant fungus $A$. niger. The study provided a better understanding of the membrane composition and topology, especially with respect to proteins with a putative transporter function; the $A$. niger transportome. Analysis of the $A$. niger in vivo transportome with HMMglut was shown to be effective; by combining the abundance patterns of the proteins identified in the experimental conditions with their respective HMMglut scores, two new putative glucose transporters, MstG and MstH, were identified. They were functionally validated in an engineered yeast strain with a monosaccharide transporter null-background and confirmed to be high affinity transporters for glucose. 


\section{Methods}

\section{In silico transportome analysis and construction of a hidden Markov model specific for glucose transporters}

The A. niger ATCC1015 and S. cerevisiae CEN.PK proteomes plus their annotations, which were used for the in silico analysis, were obtained from the JGI database [35]. For $A$. niger ATCC1015, only the best model proteome was used. Transmembrane helix domains were predicted with a standalone version of the TMHMM tool from the TMHMM website [36]. Hidden Markov models for the major facilitator superfamily (HMMMFS) and sugar porter (HMMSP) domains were obtained from the Pfam database [37]. The protein sequences used to build the HMMgluT were obtained by entering the search term "sugar transporter" in the uniprot database and downloading the resultant xml and canonical fasta files. The xml file was parsed for proteins that were not experimentally verified to have the biological function assigned to them. The remaining proteins were divided into two separate datasets; the first dataset contained only the proteins with an experimentally verified GO term related to glucose transport (GO:0005355 and GO:0015758); the second dataset contained the remaining proteins (core dataset). The protein sequences of the experimentally verified glucose transporters were aligned by accessing the PRALINE structural alignment tool [38] via the SOAP interface and using the following settings: BLOSUM62, PSI-BLAST pre-profile processing (Homology extended alignment, 3 iterations), structural features: DSSP-defined secondary structure search, PSIPRED and TMHMM, fasta output file. The aligned fasta output was converted to the Stockholm format, and the HMMgluT was built from the resultant output.sto file using the "hmmbuild" command from HMMER v3.0 [39]. A. niger MstA was excluded from the multiple alignment. For the threefold cross-validation, the initial dataset containing the 42 verified glucose transporters was randomly partitioned into three equally sized subsets. The model was then built from every combination of two subsets, while the validation of the model was carried out on the core dataset plus the third subset of the glucose transporters not used for the model building process. This allowed for the determination of false and true negatives in comparison to false and true positives, and thus gave an estimate of the prediction power of the model. The threefold cross-validation was repeated ten times, each time with different random subsets of the verified glucose transporters. Two thresholds were determined from the average ROC curve resulting from the $10 \times 3$-fold cross validation of the $\mathrm{HMM}_{\mathrm{gluT}}$ : 
1. Minimal distance to the point $\left[\begin{array}{ll}0 & 1\end{array}\right]\left(d_{\min }\right)$ :

$$
d=\sqrt{\left(1-s_{n}\right)^{2}+\left(1-s_{p}\right)^{2}}
$$

With $s_{n}=$ sensitivity (TPR) and $s_{p}=$ specificity (TNR). Another metric to calculate the optimal trade-off point between true and false predictions is the Matthews correlation coefficient. The Matthews correlation coefficient is essentially a one number representation of the confusion matrix, and is thus suitable to compare the outcome of different model predictions. This coefficient is calculated as follows:

2. Matthews correlation coefficient (MCC):

$$
M C C=\frac{T P \cdot T N-F P \cdot F N}{\sqrt{(T P+F P)(T P+F N)(T P+F P)(T N+F N)}}
$$

Here, a value closer to 1 is clearly a better prediction, and thus the inferred $\mathrm{HMM}_{\mathrm{gluT}}$ score at the highest $\mathrm{MCC}$ value calculated along the ROC curve, $\mathrm{MCC}_{\max }$, was set as second threshold. Similarly, the BLAST approach was evaluated by using each of the 42 verified glucose transporters as separate query for a local BLASTp search against the core dataset plus the other verified glucose transporters, allowing for the determination of true and false-positive rates (see Additional file 2). Strains and growth conditions Escherichia coli DH5 $\alpha$ (endA1,

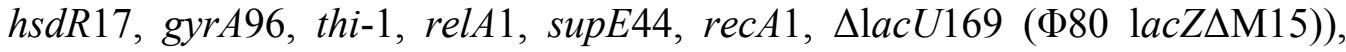
grown at $37^{\circ} \mathrm{C}$, was used for cloning experiments and plasmid propagation. Luria broth (LB) was used as growth medium $(1 \% \mathrm{w} / \mathrm{v}$ tryptone, $0.5 \% \mathrm{w} / \mathrm{v}$ yeast extract, $1 \% \mathrm{w} / \mathrm{v} \mathrm{NaCl}$ ) with or without $100 \mu \mathrm{g} \mathrm{mL} \mathrm{m}^{-1}$ ampicillin. The wild-type strain A. niger N400 (CBS 120.49), used for the plasma membrane proteomics analysis, was grown at $30^{\circ} \mathrm{C}$ on complete medium plates for spores generation and maintenance [40]. Mycelial biomass of the N400 strain was obtained after $18 \mathrm{~h}$ of growth in liquid cultures containing minimal medium, including $5 \mathrm{~g} \mathrm{~L}^{-1}$ of yeast extract, with $100 \mathrm{mM}$ sorbitol as carbon source. Equal amounts of waterrinsed mycelium were transferred to 1 -L bench top fermenters (Sartorius) with $750 \mathrm{~mL}$ of minimal medium containing $4.50 \mathrm{~g} \mathrm{NaNO}_{3}, 1.13 \mathrm{~g} \mathrm{KH}_{2} \mathrm{PO}_{4}, 0.38 \mathrm{~g} \mathrm{KCl}$, $0.38 \mathrm{~g} \mathrm{MgSO}_{4} \cdot 7 \mathrm{H}_{2} \mathrm{O}$ and $750 \mu \mathrm{L}$ of Vishniac solution [40,41]. Three different conditions, varying in the carbon source composition, were studied: sorbitol $100 \mathrm{mM}$ (reference condition), sorbitol $100 \mathrm{mM}$ plus glucose $1 \mathrm{mM}$ (low-glucose condition) and sorbitol $100 \mathrm{mM}$ plus glucose $60 \mathrm{mM}$ (high-glucose condition). Two biological replicates per condition were studied. Fermenters were stirred at $1000 \mathrm{rpm}$ and aerated with filtered air $\left(0.6 \mathrm{~L} \mathrm{~min}^{-1}\right)$, keeping oxygen levels over 
$60 \%$. The initial $\mathrm{pH}$, set at 4.0, was allowed to drop until $\mathrm{pH} 3.5$ and kept constant afterwards by sodium hydroxide addition. Mycelium samples for RT-qPCR analysis were obtained from a mycelium transfer experiment similar to the one described above, using minimal medium with the following carbon source compositions: $100 \mathrm{mM}$ sorbitol (reference condition), glucose $5 \mathrm{mM}$, glucose $55 \mathrm{mM}$, fructose $5 \mathrm{mM}$, fructose $55 \mathrm{mM}$, mannose $5 \mathrm{mM}$ and mannose $55 \mathrm{mM}$. Two biological replicates per condition were studied as well. Cultures were performed in Erlenmeyer flasks, stirred at $200 \mathrm{rpm}$ and the initial $\mathrm{pH}$ of the medium in all conditions was set at 4.0. Two hours after inoculation mycelium samples were taken and quickly washed, dried with a single-use towel, snap-frozen with liquid nitrogen and stored at $-80^{\circ} \mathrm{C}$ until further processing. The $S$. cerevisiae strain EBY.VW4000 (CEN.PK2-1C hxt134::loxP; hxt15:: LloxP; hxt164::loxP;

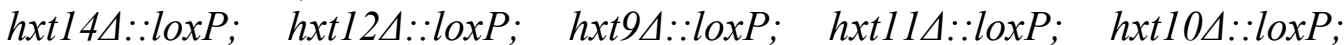

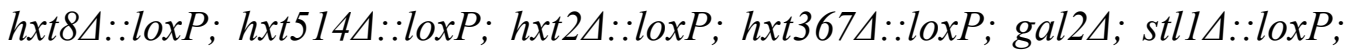

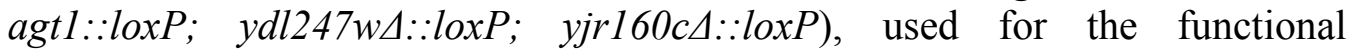
complementation experiments and the characterization of glucose transporters [12], was grown at $30^{\circ} \mathrm{C}$ and maintained on solid complete medium containing $10 \mathrm{~g} \mathrm{~L}^{-1}$ of yeast extract, $20 \mathrm{~g} \mathrm{~L}^{-1}$ of peptone and $20 \mathrm{~g} \mathrm{~L}^{-1}$ of maltose. The EBY.VW4000-derived strains obtained in the present study were grown in liquid minimal medium containing $6.7 \mathrm{~g} \mathrm{~L}^{-1}$ of yeast nitrogen base with ammonium sulphate (Difco), $20 \mathrm{~g} \mathrm{~L}^{-1}$ of maltose, supplemented with leucine $\left(30 \mathrm{mg} \mathrm{L}^{-1}\right)$, tryptophan $\left(20 \mathrm{mg} \mathrm{L}^{-1}\right)$ and histidine $\left(20 \mathrm{mg} \mathrm{L}^{-1}\right)$.

\section{A. niger membrane-associated protein purification and quality control analysis}

Aspergillus niger mycelium samples $(2-3 \mathrm{~g}$, press-dried), washed with a $20 \mathrm{mM}$ HEPES buffer $\mathrm{pH} 7.6$ containing $150 \mathrm{mM} \mathrm{NaCl}$, and resuspended in the same solution containing $1 \%(\mathrm{v} / \mathrm{v})$ protease inhibitor cocktail for yeast and fungi (Sigma), were mechanically disrupted using a French press (8000psi). Cell-free extracts were centrifuged for $5 \mathrm{~min}$ at low speed $(500 \mathrm{~g})$, in order to remove unbroken cells and pellet debris. The supernatants were then centrifuged during $20 \mathrm{~min}$ at medium speed $(5000 \mathrm{~g})$, to pellet and remove remaining heavy organelles. The remaining supernatants were centrifuged for $120 \mathrm{~min}$ at high speed $(\sim 85,000 g)$, to pellet light organelles (P3). P3 pellets were resuspended using a Dounce homogenizer in $1 \mathrm{~mL}$ of a $20 \mathrm{mM}$ HEPES buffer $\mathrm{pH} 7.6$ containing $250 \mathrm{mM}$ sucrose. $\mathrm{P} 3$ suspensions were overlaid in a discontinuous sucrose density gradient, prepared by layering successive decreasing sucrose densities solutions $(6 \mathrm{x} 1 \mathrm{~mL})$, with concentrations ranging from 1.20 to $0.70 \mathrm{M}$, upon one another. Sucrose density gradients were centrifuged $(\sim 100,000 \mathrm{~g}, 60 \mathrm{~min})$ to isolate 
different membrane-associated fractions from P3 pellet. Five fractions were obtained (P3A, P3B, P3C, P3D and P3E).

\section{Sample preparation for LC-MS/MS}

The protein content of enriched plasma membrane-associated fractions was determined using the BCA protein assay. Membrane proteins were solubilized by mixing volumes of each fraction containing $25 \mu \mathrm{g}$ of protein with equal volumes of a $2 \mathrm{x}$ solution of $20 \mathrm{mM}$ HEPES $\mathrm{pH} 7.6$ containing $1 \mathrm{M} 6$-aminocaproic acid and $10 \mathrm{~g} \mathrm{~L}^{-1}$ of $\mathrm{n}$-dodecyl-beta-D-maltoside. Cell membrane-detergent mixes were incubated in a thermoblock (Thermo- Mixer) for $1 \mathrm{~h}$ at $20^{\circ} \mathrm{C}$ and vigorous stirring (1000rpm). Afterwards, samples were sonicated in a water bath for $15 \mathrm{~min}$, and finally they were centrifuged at $22,000 \mathrm{~g}$ for $30 \mathrm{~min}$. Supernatants containing solubilized proteins were concentrated using Microcon YM-10 columns (cutoff $10 \mathrm{kDa}$; Millipore, Eschborn, Germany) and loaded into a 12\% SDSpolyacrylamide gel, which was run until the loaded samples entered into the gel. The gel was stained according to the manufacturer's instructions using Page Blue staining (Fermentas) and rinsed with ultrapure water. Each sample-gel lane was cut into one slice (approx. $1 \mathrm{~cm}^{2}$ ), carefully sliced into smaller pieces of about $1 \mathrm{~mm}^{3}$ and transferred into microcentrifuge tubes. Samples were destained and equilibrated through three washing steps using the following solutions: $50 \mathrm{mM}$ ammonium bicarbonate (ABC) (incubated $5 \mathrm{~min}), \mathrm{ABC} /$ acetonitrile $(1: 1, \mathrm{v} / \mathrm{v})$ (incubated $5 \mathrm{~min}$ ) and neat acetonitrile (incubated $5 \mathrm{~min}$ ). These washing steps were successively repeated two times. The gel samples were then swelled in 10 $\mathrm{mM}$ dithiothreitol (DTT) for $20 \mathrm{~min}$ at $56^{\circ} \mathrm{C}$ to reduce protein disulfide bonds. Subsequently, the DTT solutions were removed and samples were alkylated with $50 \mathrm{mM} 2$-chloroacetamide in $\mathrm{ABC}$, for $20 \mathrm{~min}$, at room temperature, in the dark. The 2-chloroacetamide solutions were removed, and samples were again washed twice with: neat acetonitrile (incubated $5 \mathrm{~min}$ ), $\mathrm{ABC}$ (incubated 5min) and neat acetonitrile (incubated $5 \mathrm{~min}$ ). Approximately $150 \mu \mathrm{L}$ of digestion buffer, containing sequencing grade modified trypsin $\left(12.5 \mathrm{ng} \mu \mathrm{L}^{-1}\right)$ (Promega, Madison, WI, USA) in ABC, was added to each sample, making sure that all gel pieces were kept wet during digestion (adding, if necessary, additional $\mathrm{ABC}$ solution). Protein samples were digested overnight at $37^{\circ} \mathrm{C}$. Peptide digestion products were extracted by adding $50 \mu \mathrm{L}$ of $2 \%$ trifluoroacetic acid (TFA), followed by an incubation step in a thermoblock (ThermoMixer) for $20 \mathrm{~min}$, at room temperature and vigorous stirring (1400rpm). Gel pieces were then subjected to 20 s sonication in a water bath, centrifuged and supernatants were transferred to new tubes. The peptide extraction step was then repeated once by washing the gel pieces with buffer B $(80 \%$ acetonitrile, $0.1 \%$ formic acid) followed by the mentioned 
incubation and sonication steps. Supernatants from both extractions were pooled and samples were placed in a vacuum centrifuge for acetonitrile evaporation until $20-40 \mu \mathrm{L}$ was left. Finally, samples were acidified by addition of TFA $(1: 1, \mathrm{v} / \mathrm{v})$ and peptide cleanup procedure, prior to LC-MS/MS analysis, was performed using the "STop And Go Extraction" procedure as described before [42].

\section{Mass spectrometric measurements}

LC-MS/MS analysis was performed at Radboud Proteomics Centre as described previously [43]. Measurements were performed by nanoflow reversed-phase C18 liquid chromatography (EASY nLC, Thermo Scientific) coupled online to a 7Tesla linear ion trap Fourier-Transform ion cyclotron resonance mass spectrometer (LTQ FT Ultra, Thermo Scientific).

\section{Proteomics data analysis}

The LC-MS/MS spectra obtained from the proteomics experiment were identified and quantified using the max-Quant software [44]. The peptides were mapped against the annotated A. niger ATCC1015 in silico proteome obtained from the JGI database (http://genome.jgi-psf. org/Aspni7/Aspni7.home.html) using the default settings of the maxQuant version 1.3.0.5 [variable modifications: oxidation (M) and acetylation (protein N-term); enzyme used: trypsin/P; fixed modifications carbamidomethyl (cys)], except for the variables affecting the label-free quantification. For this, the multiplicity was set to 1 , and the parameters for label-free quantification as well as the iBAQ and peak property calculations were selected. Only proteins with two or more unique peptide hits were considered for further analysis. Protein localization was determined using the Softberry protComp prediction server (http://linux1.softberry.com). The relative abundances of the identified proteins are represented as follows:

$$
\Pi=\frac{1+A_{L}}{1+A_{S}}-\frac{1+A_{H}}{1+A_{S}}
$$

where $A_{L}$ is the relative abundance of the protein in the low-glucose condition; $A_{H}$, relative abundance of the protein in the high-glucose condition; $A_{S}$, relative abundance of the protein in the reference (sorbitol) condition.

\section{Transcriptional analysis of $m s t G$ and $m s t H$ genes}

Mycelium samples were disrupted with glass beads in a Fastprep-24 instrument, and RNA extraction was performed by a Maxwell 16 instrument using the Maxwell 16 LEV simplyRNA kit (Promega). Reverse transcription and qPCR 
analysis were performed following the protocols and instruments described in Mach-Aigner et al. [45] with the primers listed in table 5. The previously described histone-like gene "hist" transcript (gene ID 207921) was used as reference for normalization of the expression data [45].

Table 5 Sequences of the primers used for qPCR analysis in this study:

\begin{tabular}{|l|l|}
\hline hist-FW & ACAATGACTGGCCGTGGAAAGG \\
\hline hist-RV & ATACGCTTGACACCACCACGAC \\
\hline mstG-FW & CGGTGGTGGTATGGCTTTCT \\
\hline mstG-RV & GTTCTCAGGCACACCGTACA \\
\hline mstH-FW & GCCATCATGATCGGTCTGTTTGTC \\
\hline mstH-RV & ACTGATGGTTCCGGTGTCATATCC \\
\hline
\end{tabular}

\section{Construction of $S$. cerevisiae EBY.WV4000 transformants expressing $A$. niger $m s t G$ and $m s t H$ genes and glucose uptake assays}

The coding sequence of the genes $m s t G$ and $m s t H$, digested with SpeI and XhoI were cloned on the $S$. cerevisiae expression vector p426HXT7-6His, previously linearized with SpeI and XhoI under the control of the constitutive promoter $\mathrm{HXT}_{\mathrm{p}}$ and the terminator $\mathrm{CYCl}_{\mathrm{t}}$. Transformation of $S$. cerevisiae EBY.WV4000 with p426HXT7-6His (empty vector), p426HXT7-6His $m s t G$ and p426HXT7-6His_mstH was performed as described [46]. Uptake assays were performed as described with minor adjustments [47]. $5 \mathrm{~mL}$ of Synthetic Complete medium without uracil (SC-ura; 0.67\% YNB (Difco)+ dropout supplement mix without uracil (Sigma-Aldrich)) with 2\% maltose as a carbon source was inoculated from plate with EBY.VW4000 with pRS426H7 (control) and EBY.VW4000 with pRS426H7_mst $G$ or pRS426H7 mst $H$ and incubated overnight $\left(30^{\circ} \mathrm{C}, 225 \mathrm{rpm}\right)$ as pre-inoculum. Pre-inoculum was transferred to $500 \mathrm{~mL}$ SC-ura with $2 \%$ maltose and incubated for $24 \mathrm{~h}$. Cells were harvested by centrifugation $(4000 \mathrm{~g}, 10 \mathrm{~min})$ and washed with $50 \mathrm{~mL}$ ice-cold MQ. Cells were then resuspended in $2 \mathrm{~mL}$ ice-cold SC-ura without carbon source, divided in $40 \mu \mathrm{L}$ aliquots and kept on ice. Aliquots were incubated for $5 \mathrm{~min}$ at $30^{\circ} \mathrm{C}$ before uptake assay was started. To start the reaction, $10 \mu \mathrm{L}$ of a five times concentrated labeled glucose solution (D - [U- $\left.{ }^{14} \mathrm{C}\right]$-glucose, Campro Scientific) was added. After exactly 10 s the reaction was stopped by the addition of $1 \mathrm{~mL}$ of $100 \mathrm{mM} \mathrm{LiCl}$ and vacuum filtration $(0.45 \mu \mathrm{m}$ HV filters, 1225 sampling manifold, Millipore), with subsequent washing with $\mathrm{x} 5 \mathrm{~mL}$ of ice-cold $100 \mathrm{mM} \mathrm{LiCl}$. After $5 \mathrm{~min}$ of drying in the vacuum manifold, the filters were transferred to scintillation vials with $7.5 \mathrm{~mL}$ scintillation liquid (Ultima Gold, Perkin Elmer) and activity was counted (Packard Tricarb 1600TR). All reactions were performed in triplicates. For each 
reaction, a negative control assay without incubation was performed. To determine transport kinetics, uptake of labeled glucose with a range of concentrations from $1 \mu \mathrm{M}$ to $10 \mathrm{mM}$ was measured in SC-ura at $\mathrm{pH} 5.0$. Glucose solutions with an activity of approximately $700-70,000 \mathrm{~Bq}$ were used. To determine kinetic parameters $K_{m}$ and $V_{\max }$, the data were fitted to the following Michaelis-Menten model with substrate inhibition using the least-squares method.

$$
V=\frac{V_{\max } \cdot[S]}{K_{m}+[S]+\frac{[S]^{2}}{K_{i}}}
$$

To determine substrate specificity, the uptake of $1 \mathrm{mM}$ of labeled glucose was measured in the presence of $10 \mathrm{mM}$ of a competing carbon source. D-glucose, Dsorbitol, D-xylose, D-mannose, L-rhamnose, L-arabinose, D-fructose and Dgalactose were selected as competing carbon sources. To determine if the transporter functions via proton symport, activity was measured while uncoupling the proton gradient by carbonyl cyanide m-chlorophenyl hydrazine (CCCP). This inhibitor is dissolved in DMSO and added before temperature equilibration of the cells. The uptake rate in the presence of $250 \mu \mathrm{M}$ of CCCP and $2 \%$ DMSO was compared with the uptake rate in the presence of only $2 \%$ DMSO. 


\section{References}

1. Andersen MR, Salazar MP, Schaap PJ, van de Vondervoort PJI, Culley D, Thykaer J, et al. Comparative genomics of citric-acid-producing Aspergillus niger ATCC 1015 versus enzyme-producing CBS 513.88. Genome .... 2011. 2. de Souza WR, Maitan-Alfenas GP, de Gouvêa PF, Brown NA, Savoldi M, Battaglia E, et al. The influence of Aspergillus niger transcription factors AraR and XlnR in the gene expression during growth in D-xylose, L-arabinose and steam-exploded sugarcane bagasse. Fungal Genet. Biol. [Internet]. 2013;60:387-97. Available from:

http://eutils.ncbi.nlm.nih.gov/entrez/eutils/elink.fcgi?dbfrom=pubmed\&id=214 84208\&retmode $=$ ref\&cmd $=$ prlinks

3. Tamayo-Ramos JA, Barends S, de Lange D, de Jel A, Verhaert R, de Graaff L. Enhanced production of Aspergillus nigerlaccase-like multicopper oxidases through mRNA optimization of the glucoamylase expression system. Biotechnol. Bioeng. 2012;110:543-51.

4. de Vries RP, Visser J. Aspergillus Enzymes Involved in Degradation of Plant Cell Wall Polysaccharides. Microbiology and Molecular Biology Reviews. 2001;65:497-522.

5. van Peij NNME, Gielkens MMC, de Vries RP, Visser J, de Graaff LH. The Transcriptional Activator XlnR Regulates Both Xylanolytic and Endoglucanase Gene Expression in Aspergillus niger. Applied and Environmental Microbiology. 1998.

6. Battaglia E, Hansen SF, Leendertse A, Madrid S, Mulder H, Nikolaev I, et al. Regulation of pentose utilization by AraR, but not XlnR, differs in Aspergillus nidulans and Aspergillus niger. Appl Microbiol Biotechnol. 2011;91:387-97. 7. Gruben BS, Zhou M, Wiebenga A, Ballering J, Overkamp KM, Punt PJ, et al. Aspergillus niger RhaR, a regulator involved in 1-rhamnose release and catabolism. Appl Microbiol Biotechnol. Springer Berlin Heidelberg; 2014;:110 .

8. Torres NV, Riol-Cimas JM, Wolschek M, Kubicek CP. Glucose transport by Aspergillus niger: the low-affinity carrier is only formed during growth on high glucose concentrations. Appl Microbiol Biotechnol. 1996;44:790-4.

9. Jørgensen TR, vanKuyk PA, Poulsen BR, Ruijter GJG, Visser J, Iversen JJL. Glucose uptake and growth of glucose-limited chemostat cultures of Aspergillus niger and a disruptant lacking MstA, a high-affinity glucose transporter. Microbiology (Reading, Engl.). 2007;153:1963-73.

10. Coelho MA, Gonçalves C, Sampaio JP, Gonçalves P. Extensive intrakingdom horizontal gene transfer converging on a fungal fructose transporter gene. PLoS Genet. 2013;9:e1003587. 
11. Leandro MJ, Fonseca C, Gonçalves P. Hexose and pentose transport in ascomycetous yeasts: an overview. FEMS Yeast Research. 2009;9:511-25. 12. Wieczorke R, Krampe S, Weierstall T, Freidel K, Hollenberg CP, Boles E. Concurrent knock-out of at least 20 transporter genes is required to block uptake of hexoses in Saccharomyces cerevisiae. FEBS Letters. 1999;464:1238.

13. Reifenberger E, Freidel K, Ciriacy M. Identification of novel HXT genes in Saccharomyces cerevisiae reveals the impact of individual hexose transporters on qlycolytic flux. Mol Microbiol. 1995;16:157-67.

14. vanKuyk PA, Diderich JA, MacCabe AP, Hererro O, Ruijter G, Visser J. Aspergillus niger mstA encodes a high-affinity sugar $/ \mathrm{H}+$ symporter which is regulated in response to extracellular pH. Biochem. J. 2004;379:375-83.

15. Polidori E, Ceccaroli P, Saltarelli R, Guescini M, Menotta M, Agostini D, et al. Hexose uptake in the plant symbiotic ascomycete Tuber borchii Vittadini: biochemical features and expression pattern of the transporter TBHXT1. Fungal Genetics and Biology. 2007;44:187-98.

16. Saloheimo A, Rauta J, Stasyk OV, Sibirny AA, Penttila M, Ruohonen L. Xylose transport studies with xylose-utilizing Saccharomyces cerevisiae strains expressing heterologous and homologous permeases. Appl Microbiol Biotechnol. Springer-Verlag; 2006;74:1041-52.

17. Du J, Li S, Zhao H. Discovery and characterization of novel d-xylose specific transporters from Neurospora crassa and Pichia stipitis. Molecular BioSystems. Royal Society of Chemistry; 2010;6:2150-6.

18. Wahl R, Wippel KGSKMJRSN. A Novel High-Affinity Sucrose

Transporter Is Required for Virulence of the Plant Pathogen Ustilago maydis [electronic resource]. Public Library of Science.

19. Leandro MJ, Sychrová H, Prista C, Loureiro-Dias MC. ZrFsy1, a HighAffinity Fructose/H+ Symporter from Fructophilic Yeast Zygosaccharomyces rouxii. PLoS ONE. 2013;8.

20. Reis dos TF, Menino JF, Bom V, Brown NA. PLOS ONE: Identification of Glucose Transporters in Aspergillus nidulans. PLoS ONE. 2013.

21. Colabardini A, Ries LN, Brown N, Reis dos T, Savoldi M, Goldman MHS, et al. Functional characterization of a xylose transporter in Aspergillus nidulans. Biotechnol Biofuels. 2014;7:46.

22. Sloothaak J, Schilders M, Schaap PJ, de Graaff LH. Overexpression of the Aspergillus niger GatA transporter leads to preferential use of D-galacturonic acid over D-xylose. AMB Express. 2014;4:66.

23. Martens-Uzunova ES, Schaap PJ. An evolutionary conserved Dgalacturonic acid metabolic pathway operates across filamentous fungi capable 
of pectin degradation. Fungal Genetics and Biology [Internet]. 2008;45:144957. Available from:

http://www.sciencedirect.com/science/article/pii/S1087184508001576 24. Vardy E, Arkin IT, Gottschalk KE, Kaback HR, Schuldiner S. Structural conservation in the major facilitator superfamily as revealed by comparative modeling. Protein Science. 2004;13:1832-40.

25. Eddy SR. Profile hidden Markov models. Bioinformatics. 1998;14:755-63. 26. Kim H, Melen K, Osterberg M, Heijne von G. A global topology map of the Saccharomyces cerevisiae membrane proteome. Proceedings of the National Academy of Sciences. National Academy of Sciences; 2006;103:11142-7. 27. Delom F, Szponarski W, Sommerer N, Boyer JC, Bruneau JM, Rossignol $\mathrm{M}$, et al. The plasma membrane proteome of Saccharomyces cerevisiae and its response to the antifungal calcofluor. Proteomics. 2006;6:3029-39.

28. Szopinska A, Degand H, Hochstenbach J-F, Nader J, Morsomme P. Rapid Response of the Yeast Plasma Membrane Proteome to Salt Stress. Mol. Cell Proteomics. 2011;10.

29. Cabezon V, Llama-Palacios A, Nombela C, Monteoliva L, Gil C. Analysis of Candida albicans plasma membrane proteome. Proteomics. 2009;9:4770-86. 30. Ouyang H, Luo Y, Zhang L, Li Y, Jin C. Proteome Analysis of Aspergillus fumigatus Total Membrane Proteins Identifies Proteins Associated with the Glycoconjugates and Cell Wall Biosynthesis Using 2D LC-MS/MS. Mol. Biotechnol. 2010;44:177-89.

31. Rogers PD, Vermitsky J-P, Edlind TD, Hilliard GM. Proteomic analysis of experimentally induced azole resistance in Candida glabrata. J. Antimicrob. Chemother. 2006;58:434-8.

32. de Oliveira JMPF, van Passel MWJ, Schaap PJ, de Graaff LH. Shotgun proteomics of Aspergillus niger microsomes upon D-xylose induction. Applied and Environmental Microbiology. 2010;76:4421-9.

33. de Oliveira JMPF, van Passel MWJ, Schaap PJ, de Graaff LH. Proteomic analysis of the secretory response of Aspergillus niger to D-maltose and Dxylose. PLoS ONE. 2011;6:e20865.

34. Patel VJ, Thalassinos K, Slade SE, Connolly JB, Crombie A, Murrell JC, et al. A comparison of labeling and label-free mass spectrometry-based proteomics approaches. J. Proteome Res. 2009;8:3752-9.

35. Krogh A, Larsson B, von Heijne G, Sonnhammer EL (2001) Predicting transmembrane protein topology with a hidden Markov model: application to complete genomes. J Mol Biol 305:567-580

36. Pao SS, Paulsen IT, Saier MH (1998) Major facilitator superfamily. Microbiol Mol Biol Rev 62:1-34 
37. Punta M, Coggill PC, Eberhardt RY, Mistry J, Tate J, Boursnell C, Pang N, Forslund K, Ceric G, Clements J, Heger A, Holm L, Sonnhammer ELL, Eddy SR, Bateman A, Finn RD (2012) The Pfam protein families database. Nucleic Acids Res 40(Database issue):D290-D301

38. UniProt Consortium (2014) UniProt: a hub for protein information. Nucleic Acids Res 43(Database issue):D204-D212

39. Zeng H, Parthasarathy R, Rampal AL, Jung CY (1996) Proposed structure of putative glucose channel in GLUT1 facilitative glucose transporter. Biophys J 70:14-21

40. Deng D, Xu C, Sun P, Wu J, Yan C, Hu M, Yan N (2014) Crystal structure of the human glucose transporter GLUT1. Nature 510:121-125

41. Strauss J, Horvath HK, Abdallah BM, Kindermann J, Mach RL, Kubicek

CP (1999) The function of CreA, the carbon catabolite repressor of Aspergillus nidulans, is regulated at the transcriptional and post-transcriptional level. Mol Microbiol 32:169-178

42. De Oliveira JMPF, de Graaff LH (2011) Proteomics of industrial fungi: trends and insights for biotechnology. Appl Microbiol Biotechnol 89:225-237 43. Ozcan S, Johnston M (1999) Function and regulation of yeast hexose transporters. Microbiol Mol Biol Rev 63:554-569

44. Forment JV, Flipphi M, Ventura L, González R, Ramón D, MacCabe AP (2014) High-affinity glucose transport in Aspergillus nidulans is mediated by the products of two related but differentially expressed genes. PLoS One 9:e94662

45. Van Leeuwen MR, Krijgsheld P, Wyatt TT, Golovina EA, Menke H, Dekker A, Stark J, Stam H, Bleichrodt R, Wösten HAB, Dijksterhuis J (2013) The effect of natamycin on the transcriptome of conidia of Aspergillus niger. Stud Mycol 74:71-85

46. Walsh MC, Smits HP, Scholte M, van Dam K (1994) Affinity of glucose transport in Saccharomyces cerevisiae is modulated during growth on glucose. $\mathrm{J}$ Bacteriol 176:953-958

47. Maier A, Völker B, Boles E, Fuhrmann GF (2002) Characterization of glucose transport in Saccharomyces cerevisiae with plasma membrane vesicles (countertransport) and intact cells (initial uptake) with single Hxt1, Hxt2, Hxt3, Hxt4, Hxt6, Hxt7 or Gal2 transporters. FEMS Yeast Res 2:539-550 48. Nordberg H, Cantor M, Dusheyko S, Hua S, Poliakov A, Shabalov I, Smirnova T, Grigoriev IV, Dubchak I (2014) The genome portal of the Department of Energy Joint Genome Institute: 2014 updates. Nucleic Acids Res 42((Database issue)):D26-D31 
49. Simossis VA, Heringa J (2005) PRALINE: a multiple sequence alignment toolbox that integrates homology-extended and secondary structure information. Nucleic Acids Res 33(Web Server issue):W289-W294 50. Finn RD, Clements J, Eddy SR (2011) HMMER web server: interactive sequence similarity searching. Nucleic Acids Res 39(Web Server issue):W29W37

51. Pontecorvo G, Roper JA, Chemmons LM, Macdonald KD, Bufton AWJ (1953) The genetics of Aspergillus nidulans. Adv Genet 5:141-238

52. Vishniac W, Santer M (1957) The thiobacilli. Bacteriol Rev 21:195-213 53. Rappsilber J, Ishihama Y, Mann M (2003) Stop and go extraction tips for matrix-assisted laser desorption/ionization, nanoelectrospray, and LC/MS sample pretreatment in proteomics. Anal Chem 75:663-670

54. Rajala N, Hensen F, Wessels HJCT, Ives D, Gloerich J, Spelbrink JN (2015) Whole cell formaldehyde cross-linking simplifies purification of mitochondrial nucleoids and associated proteins involved in mitochondrial gene expression. PLoS One 10:e116726

55. Cox J, Mann M (2008) MaxQuant enables high peptide identification rates, individualized p.p.b.-range mass accuracies and proteome-wide protein quantification. Nat Biotechnol 26:1367-1372

56. Mach-Aigner AR, Omony J, Jovanovic B, van Boxtel AJB, de Graaff LH (2012) d-Xylose concentration-dependent hydrolase expression profiles and the function of CreA and XlnR in Aspergillus niger. Appl Environ Microbiol 78:3145-3155

57. Gietz RD, Woods RA (2002) Transformation of yeast by lithium acetate/singlestranded carrier DNA/polyethylene glycol method. Methods Enzymol 350:87-96 


\section{Chapter 4}

\section{Identification and functional characterization of novel xylose transporters from the cell factories Aspergillus niger and Trichoderma reesei}

Jasper Sloothaak ${ }^{\dagger}$, Juan Antonio Tamayo-Ramos ${ }^{\dagger}$, Dorett I. Odoni, Thanaporn Laothanachareon, Christian Derntl, Astrid R. Mach-Aigner, Vitor A. P. Martins dos Santos and Peter J. Schaap

\footnotetext{
${ }^{\dagger}$ These authors contributed equally to this work
}

Biotechnology for Biofuels (2016) Volume 9 


\title{
Identification and functional characterization of novel xylose transporters from the cell factories Aspergillus niger and Trichoderma
} reesei

\begin{abstract}
Global climate change and fossil fuels limitations have boosted the demand for robust and efficient microbial factories for the manufacturing of bio-based products from renewable feedstocks. In this regard, efforts have been done to enhance the enzyme-secreting ability of lignocellulose-degrading fungi, aiming to improve protein yields while taking advantage of their ability to use lignocellulosic feedstocks. Access to sugars in complex polysaccharides depends not only on their release by specific hydrolytic enzymes, but also on the presence of transporters capable of effectively transporting the constituent sugars into the cell. This study aims to identify and characterize xylose transporters from Aspergillus niger and Trichoderma reesei, two fungi that have been industrially exploited for decades for the production of lignocellulose-degrading hydrolytic enzymes.
\end{abstract}

A hidden Markov model for the identification of xylose transporters was developed and used to analyze the A. niger and T. reesei in silico proteomes, yielding a list of candidate xylose transporters. From this list, three $A$. niger (XltA, XltB and XltC) and three T. reesei (Str1, Str2 and Str3) transporters were selected, functionally validated and biochemically characterized through their expression in a Saccharomyces cerevisiae hexose transport null mutant, engineered to be able to metabolize xylose but unable to transport this sugar. All six transporters were able to support growth of the engineered yeast on xylose but varied in affinities and efficiencies in the uptake of the pentose. Amino acid sequence analysis of the selected transporters showed the presence of specific residues and motifs recently associated to xylose transporters. Transcriptional analysis of $A$. niger and $T$. reesei showed that XltA and Str1 were specifically induced by xylose and dependent on the $\mathrm{X} \operatorname{lnR} / \mathrm{Xyr} 1$ regulators, signifying a biological role for these transporters in xylose utilization.

This study revealed the existence of a variety of xylose transporters in the cell factories $A$. niger and $T$. reesei. The particular substrate specificity and biochemical properties displayed by $A$. niger XltA and XltB suggested a possible biological role for these transporters in xylose uptake. New insights were also 
gained into the molecular mechanisms regulating the pentose utilization, at inducer uptake level, in these fungi. Analysis of the A. niger and T. reesei predicted transportome with the newly developed hidden Markov model showed to be an efficient approach for the identification of new xylose transporting proteins. 


\section{Background}

Industrial production of chemicals and enzymes synthesized by fungi comprises a huge international market [1-3] and filamentous fungi such as Aspergillus niger and Trichoderma reesei have become two of the main workhorses of today's industrial biotechnology. Their high enzyme secretory capacity has already been industrially exploited during the last decades and both species have the capacity to efficiently degrade and utilize second-generation lignocellulosic feedstocks [4]. Global climate change, fossil fuels limitations and breakthroughs in the advanced biofuels market have boosted the demand for robust and efficient microbial cell factories. In this regard, to enhance the enzyme-secreting ability of these fungi, efforts at multiple levels have been done: from studies at system level to understand the secretory process, to the use of more applied strategies focused on the improvement of protein yields [5, 6]. Nevertheless, access to sugars released from the complex polysaccharides is not only dependent on the ability of these fungi to secrete high titers of a complex mix of hydrolytic enzymes, but also on the presence of a large array of sugar porters, transport proteins that are capable of effectively transporting the constituent sugars into the cell. Most of the current knowledge on sugar transporters in fungi comes from studies in the model organism Saccharomyces cerevisiae. S. cerevisiae is able to consume a limited set of mono- and disaccharides [7]. When the in silico proteome is explored with Pfam profile hidden Markov models (HMM) [8] for different transporter proteins, 73 proteins may be classified as major facilitator superfamily (MFS, Pfam ID: PF07690) transporters, of which 43 belong to the sugar porter (SP, Pfam ID: PF00083) subfamily. The superior capacity of lignocellulose- degrading fungi for sugar uptake seems clear from the number of sugar porters that are available. In the $A$. niger in silico proteome 469 proteins may be classified as MFS transporters of which 256 are included in the SP subfamily [9]. Using the same approach, we estimate that for T. reesei 235 proteins can be classified as MFS transporters of which 113 can be classified as SP. To completely eliminate the ability of $S$. cerevisiae to use glucose as a carbon source $20 \mathrm{SP}$ genes needed to be knocked-out [10]. This apparent redundancy enables the organisms to efficiently take up the available carbon source in response to a wide variety of conditions. In yeast, not only sugar transporters contribute to this flexibility, but also a number of sensors that share with these transporters the same domain architecture, but have an additional domain to connect to intracellular signal transduction pathways [11,12]. While glucose is always the preferred substrate, many of these yeast transporters have the ability to transport several other sugars including natively non-fermentable substrates like xylose. Members of the sugar porter subfamily are structurally similar in 
design, with 12 transmembrane segments organized in two distinct domains [13, 14] and only a limited number of amino acid side chains are believed to control binding and affinity $[15,16]$. In search for more efficient xylose transporters, recent studies have focused on changing the functionality of known yeast transporters and it has been shown that it is possible to change the uptake rate, affinity and inhibition through a limited set of mutations [17-21]. Native industrially relevant xylose transporters that show high affinity and capacity for xylose uptake have been identified from Escherichia coli, Pichia stipitis and Candida intermedia [22-24]. Additionally, several transporters from filamentous fungi have been reported to transport xylose, such as An25 and An29-2 from Neurospora crassa, XtrD from A. nidulans, MstA from A. niger and Xlt1 and Str1 from T. reesei [25-28]. Of this list, only An25 and MstA have been biochemically characterized. $A$. niger and $T$. reesei are natural xylose metabolizers. The aim of this study was to study their modus operandi in xylose transport. This was done through the identification, characterization and regulation analysis of a novel set of native transporters able to use xylose as a substrate. To select promising new candidates, a hidden Markov model specific for xylose transporters $\left(\mathrm{HMM}_{\mathrm{xylT}}\right)$ was developed, similarly to the HMM developed for glucose transporters [9]. With HMMxylT, the in silico proteomes of $A$. niger and $T$. reesei were mined for candidate xylose transporters and from each species three candidate xylose transporters were selected for experimental validation and biochemical characterization. All six transporters were able to transport xylose and between them they showed remarkable differences in terms of transcriptional expression regulation and substrate affinity and selectivity. 


\section{Results and discussion}

\section{A. niger and $T$, reesei in silico proteome mining}

Proteins belonging to the SP family are structurally similar, but share a low level of sequence identity [9] and only a limited number of amino acid side chains are believed to be involved in binding and affinity $[15,16]$. As discussed in our previous work, HMMs can be used to more effectively segregate SP proteins based on their substrate than standard BLAST-based methods [9]. However, precision will largely depend on the availability of a consistent training set of previously characterized proteins with the function of interest. Given the data available in literature, we aimed to build a profile HMM as specific for xylose transporters as possible. To this end, we retrieved protein sequences from 24 functionally validated transporters that are able to transport xylose, with the additional requirement that they must originate from species that are naturally able to metabolize xylose (Additional file 1). Note that, when biochemically characterized, most of these xylose transporters either performed (at least) equally well on glucose, or were inhibited by the hexose, and thus the list is far from ideal. Nonetheless, we expected that residues fundamental for xylose transport are conserved in these proteins, ultimately making them xylose transporters as well. The HMMxyl built from these sequences was used to mine the in silico proteomes of $A$. niger and $T$. reesei for putative xylose transporters. The complete workflow applied for the selection of candidate xylose transporters is presented in figure 1, Additional files 2 and 3 provide details on HMM xylT. The output list of top-scoring $A$. niger and $T$. reesei proteins was analyzed (Additional file 4), using the available literature, to search for the most promising candidates for xylose utilization. In this regard, the A. niger HMMxylT output was compared with different transcriptome analysis studies where $A$. niger was grown in the presence of xylose, xylose-arabinose mixes, sugarcane bagasse, straw or willow [29-33]. The combined results of the HMMху1т output and transcriptome analyzes indicated that many of the new $A$. niger candidate transporters with a high HMM xylT score were transcriptionally upregulated in, at least, one of these culture conditions (Additional file 5). Moreover, many of them were also possibly regulated by the $\mathrm{X} \operatorname{lnR}$ and AraR transcription factors, which control hemicellulose utilization in A. niger [32]. This suggested that the top-scoring sugar porters within the A. niger HMMxylT list contained a number of good candidates for further analysis. In the case of the $T$. reesei top-scoring sugar porters, only a few transcriptomics studies highlighting the expression modulation of hypothetical sugar transporters in hemicellulose degradation conditions are available. 

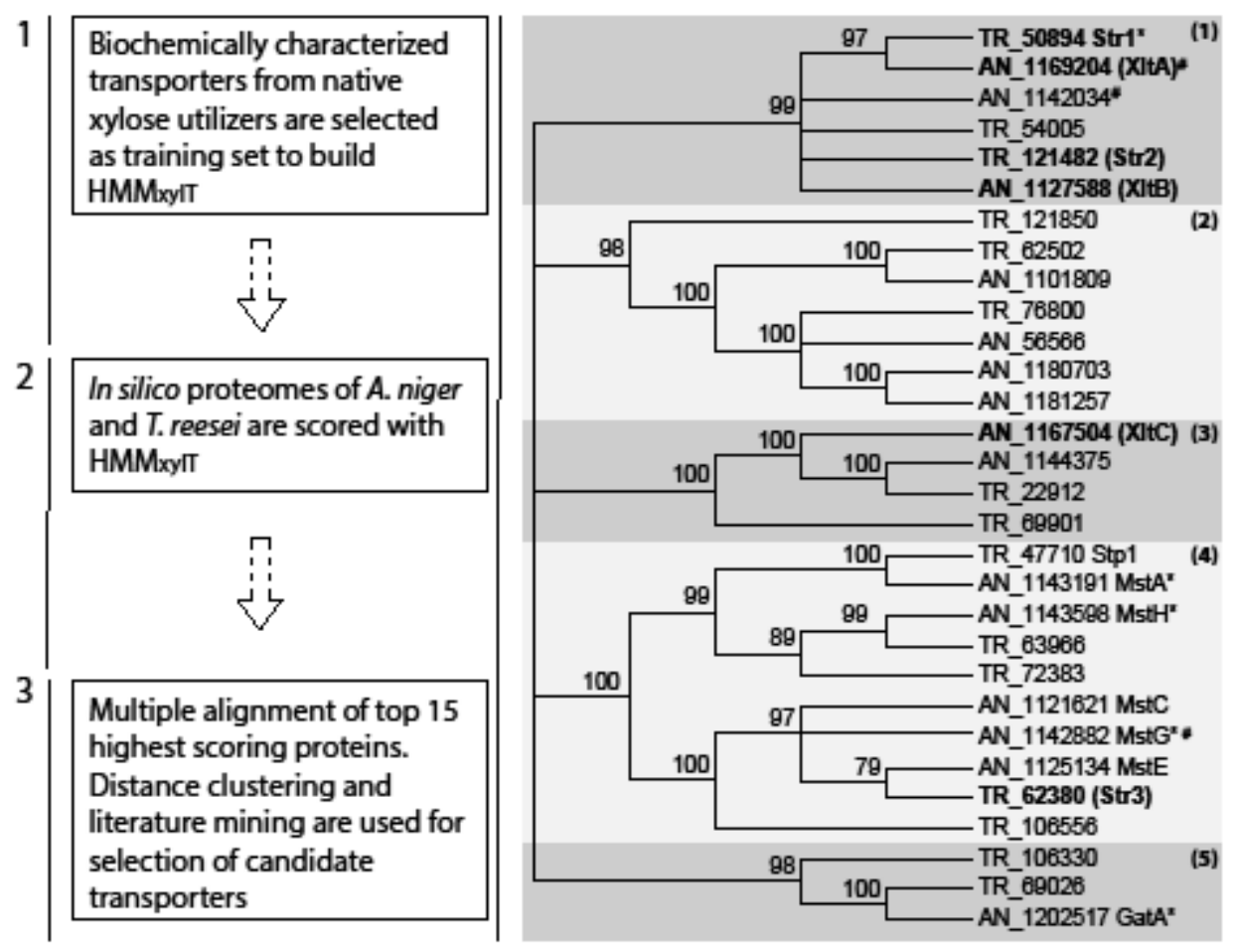

Figure 1 Approach for selection of candidate transporters (steps 1, 2 and 3), and neighbor-joining distance tree of top 15 highest $\mathrm{HMM}_{\text {xylT }}$ scoring proteins from $A$. niger and T. reesei. Functionally validated xylose transporters were retrieved to train a $\mathrm{HMM}_{\mathrm{xylT}}$, and used to analyze the in silico proteomes of $A$. niger and T. reesei. An analysis of the phylogenetic relationships of the top-scoring candidate transporters, taking into account the available literature, allowed the selection of candidate transporters that were subsequently characterized. Bootstrap values are indicated, nodes with bootstrap values $<75 \%$ are collapsed. Numbers indicate protein ID in JGI online genomes of A. niger ATCC1015 and T. reesei v2.0 [50, 66]. Proteins selected for further biochemical characterization are indicated in bold with protein names in brackets. $\mathrm{AN}_{-}, A$. niger; TR_, T. reesei; asterisks, previously experimentally validated transporters; hash symbol, the presence of a XlnR-binding motif as suggested by van Peij et al. [67] in the 1-kb upstream region of the encoding gene

Still, in two recently published studies, analyzing the $T$. reesei transcriptional response to wheat straw, many of the HMMxylT top-scoring transporters were upregulated on this carbon source versus glucose $[34,35]$. We also found that the function of three of the topscoring transporters had been studied previously [25, $28,36,37]$. One of them, TrStr1 (protID 50894) [28] was confirmed to function 
as xylose transporter, while Stp1 (protID 47710) and TrHxt1 (protID 22912) were associated to cellobiose and glucose utilization, respectively $[36,37]$.

\section{Phylogenetic clustering of top-scoring $A$. niger and $T$. reesei transporters} With the help of HMMxylT, a number of candidate xylose transporters from $A$. niger and T. reesei could be identified. Further analysis of the HMMxylт output showed that the possible function of many high-scoring transporters could be linked to hemicellulose-associated sugars, particularly in A. niger. For this reason, three top $10 \mathrm{~A}$. niger transporters AnXltA (protID 1169204), AnXltB (protID 1127588) and AnXltC (protID 1167504) were selected for further studies. For the selection of $T$. reesei xylose transporter candidates the top 15 highest scoring proteins from $A$. niger and $T$. reesei were aligned and a neighborjoining distance tree was built from the pairwise distances (Figure 1). Broadly, five protein clusters were observed. AnXltA and AnXltB are in cluster 1 and within this cluster $A$. niger XltA showed a strong clustering with $T$. reesei $\operatorname{Str} 1$ (protID 50894). Strl was recently confirmed to be essential for pentose utilization and relevant in the induction of the hemicellulose utilization system [28], and since this transporter was not yet biochemically characterized it was selected for further study. In the same cluster, T. reesei protein $121482(\operatorname{TrStr} 2)$ appeared to be a close homolog of $A$. niger XltB and thus TrStr2 was also selected for further analysis. Several transporters from cluster 4 were previously studied, showing the relevance of this subgroup for sugar uptake $[9,37,38]$. Taking this into account, and the fact that MstA has been shown to have high affinity toward xylose, we decided to select another $T$. reesei representative of this cluster (TrStr3, ProtID 62380) for further studies.

\section{Engineering of a laboratory-evolved yeast strain for functional validation of xylose transporters}

Saccharomyces cerevisiae EBY.VW4000 is a strain unable to transport glucose and is frequently used for functional validation of sugar transporters [10]. For the purpose of ex vivo functional validation of the selected fungal transporters, strain EBY.VW4000 was genetically modified to be able to metabolize xylose as a carbon source, and laboratory-evolved for an enhanced growth on xylose. For this, strain EBY.VW4000, unable to grow on glucose, mannose, galactose or fructose as carbon source, was transformed with the plasmid pRH315, expressing the $P$. stipitis d-xylose reductase (XYL1) and xylitol dehydrogenase (XYL2) genes, and the $S$. cerevisiae xylulokinase (XKS1) gene [39]. An isolated transformant of the EBY. VW.4000 strain expressing the xylose utilization pathway (EBY.XP) was subsequently transformed with a plasmid expressing the 


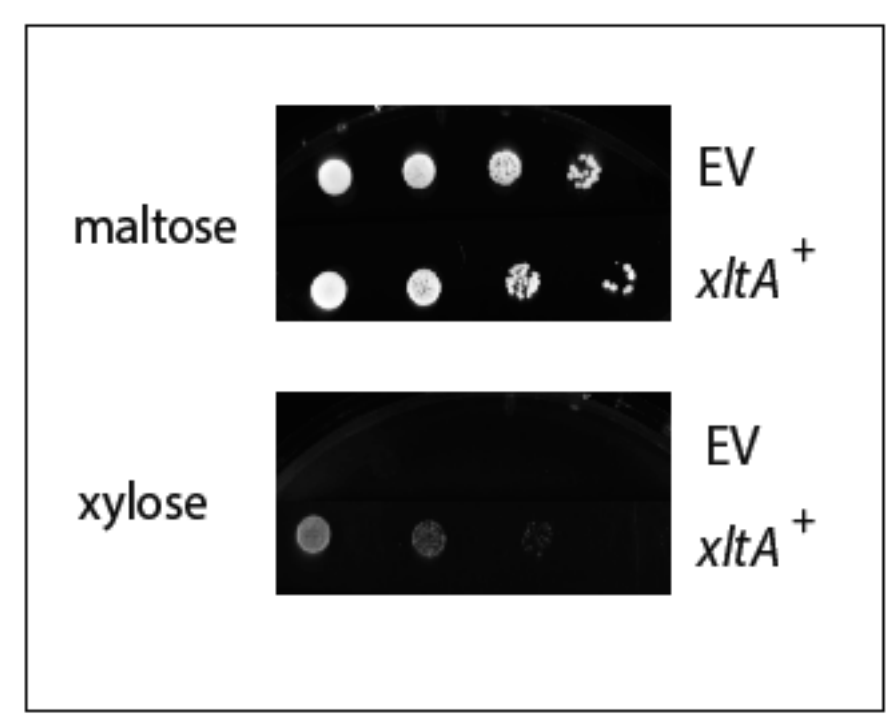

Figure 2 Functional validation of $A$. niger XltA as a xylose transporter, and of yeast strain EBY.XP as a xylose-utilizing strain. Serial dilutions of the EBY.XP $x l t A+$ strain, expressing the A. niger sugar porter gene cloned in expression vector p426HXT7-6His, were grown in minimal medium agar plates containing maltose or xylose $1 \%(w / v)$. Agar plates were incubated at $30^{\circ} \mathrm{C}$ for 5 days. EV: EBY.XP, carrying the empty expression vector p426HXT7-6His, was used as a negative control

A. niger hypothetical xylose transporter XltA (p426HXT7-6His-xltA). EBY.XP xltA transformants were isolated and the ability of XltA to confer them the capacity to grow in the presence of xylose was studied. Tenfold serial dilutions of exponentially growing cells from an xltA transformant were spotted on different minimal medium plates supplemented with either $1 \%(w / v)$ maltose or $1 \%$ $(w / v)$ xylose. After an incubation period of 5 days, the $x l t A$ transformant strain showed ability to grow on xylose (Figure 2). While this result confirmed that $A$. niger XltA is able to transport xylose, the ability of the transformant strain to grow on the pentose was poor. Poor growth of xylose-utilizing yeast transformant strains has been described before $[25,27,28]$, and this could be due to metabolic imbalances as a result of different cofactor specificities of the heterologous genes XYL1 and XYL2 present in the modified $S$. cerevisiae strain [39]. To obtain a better growing xylose-utilizing host for routine identification of xylose transporters, the ability of the EBY.XP strain to metabolize xylose was improved through laboratoryevolution. For this, the strain was grown in agar plates containing selective minimal medium with $2 \%(w / v)$ xylose, during long-term incubations (at least 2 weeks), at $30{ }^{\circ} \mathrm{C}$. Once growing colonies were observed, they were pooled and re-plated again in $2 \%(w / v)$ xylose plates. After two re-plating rounds, several single colonies showing relatively fast growth were selected and re-plated individually. Finally, a thus evolved strain showing the fastest growth, called Ag11, was selected for further research. Prior to further use as an optimized host 
for identification of new xylose transporters through functional complementation of transport, the expression vector carrying the $A$. niger xltA gene was cured from the Ag11 strain (see "Methods" section for details). The cured strain Ag11C3 was confirmed by its inability to grow in the absence of uridine, and in the presence of xylose as a single carbon source. To confirm that laboratory-evolved strain Ag11C3 could be successfully used as a host for the identification of new xylose transporters, the strain was retransformed with the p426HXT7-6His-xltA plasmid. Two Ag11C3-derived control strains were also constructed, one carrying the empty vector p426HXT7-6His, and one carrying the plasmid p426HXT7-6His-mst $G$, expressing a recently characterized glucose transporter from A. niger [9]. Four transformants per strain were isolated and, as can be observed in figure 3, only transformants carrying the p426HXT7-6His-xltA plasmid were able to grow on xylose. This result confirmed that the laboratoryevolved strain $\mathrm{Ag} 11 \mathrm{C} 3$ can be used as host for the identification of transporters able to transport xylose. It also showed that the recently identified A. niger MstG glucose transporter is unable to support growth on xylose in this strain.

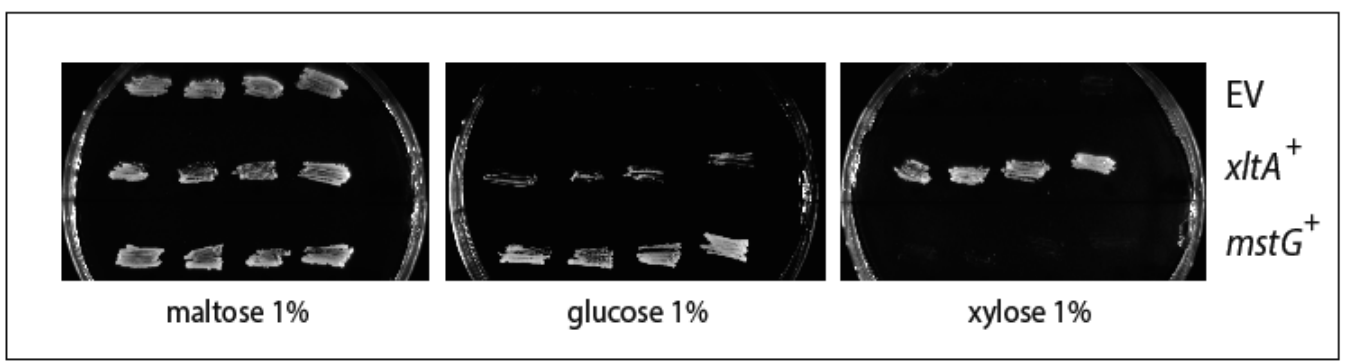

Figure 3 Functional validation of $\mathrm{Ag} 11 \mathrm{C} 3$, a laboratory-evolved growth optimized derivative of the yeast strain EBY.XP. Growth of strain Ag11C3 expressing the A. niger xltA or mstG genes; or harboring the empty expression vector p426HXT7-6His (EV), in minimal medium agar plates containing maltose, glucose, or xylose $1 \%$ $(w / v)$. Four transformants per genetic background were tested. Agar plates were incubated at $30^{\circ} \mathrm{C}$ for 7 days

Functional validation of $A$. niger AnXItA-C and T. reesei TrStr1-3 in yeast Strain $\mathrm{Ag} 11 \mathrm{C} 3$ was used to study the function of the selected $A$. niger and $T$. reesei sugar porters. Plasmids expressing $x l t B, x l t C$, strl, str 2 or str 3 were constructed using the p426HXT7-6His plasmid backbone and used to transform Ag11C3. Single-colony transformants were isolated from minimal medium agar plates containing $2 \%$ maltose and the ability of XltA, XltB, XltC, Str1, Str2 and $\mathrm{Str} 3$ to support growth of the $\mathrm{Ag} 11 \mathrm{C} 3$ transformant strains in the presence of xylose and in the presence of a number of other monosaccharides was studied 
using a plate assay. Tenfold serial dilutions of exponentially growing transformant cells were spotted on minimal medium plates supplemented with 1 $\%(w / v)$ or $0.1 \%(w / v)$ of one of the following carbon sources: xylose, glucose, fructose, galactose, mannose and maltose (Figure 4). All six previously selected transporters were functional as xylose transporters, as they all provided the Ag11C3 strain the ability to grow in xylose. Remarkable differences in the growth levels of the various transformants were observed, suggesting that the affinity of the transporters toward the pentose could be different. XltA, XltC and Str3 showed a good growth level in both high $(1 \% ; w / v)$ and low $(0.1 \% ; w / v)$ concentrations of xylose after 7 days, XltB and Str2 transformants showed a lower growth level in both xylose concentrations after the same incubation period, while growth of the Str1 transformant could only be clearly observed after a prolonged incubation time of 12 days. In addition, most of the transporters showed the ability to transport other monosaccharides. XltC, Str2 and Str3 were able to support growth of the Ag11C3 strain in the presence of all sugars tested, showing them to be the transporters with the broadest substrate specificity. XltA and Str1 showed a poor performance in the use of fructose as substrate but restored growth of $\mathrm{Ag} 11 \mathrm{C} 3$ in the presence of glucose, galactose and mannose. The most remarkable substrate utilization profile was that of the Ag11C3-XltB transformant, which was only able to grow in the presence of xylose. For a better insight in individual xylose affinities, the growth rates of transformants expressing of XltA, XltB, XltC, Str1, Str2 and Str3 were studied in liquid cultures in the presence of xylose $(0.5 \% ; w / v)$ as sole carbon source, and in the presence of a mixture of xylose $(0.5 \% ; w / v)$ and glucose $(0.5 \% ; w / v)$ (Table 1). Additional file 6 shows growth curves of the transformant strains grown in minimal medium with the described sugar compositions.

Table 1 Specific growth rate and sugar consumption by Ag11C3 transformants expressing XltA, XltB, XltC, Str1, Str2 and Str3

\begin{tabular}{|c|c|c|c|c|c|}
\hline \multirow[b]{2}{*}{$\begin{array}{l}\mathrm{Ag} 11 \mathrm{C} 3 \\
\text { strain }\end{array}$} & \multicolumn{2}{|l|}{ Xylose cultures } & \multicolumn{3}{|c|}{ Xylose + glucose cultures } \\
\hline & $\mu\left(\mathrm{h}^{-1}\right)$ & $\begin{array}{l}\text { Total xylose } \\
\text { consumed (\%) }\end{array}$ & $\mu\left(\mathrm{h}^{-1}\right)$ & $\begin{array}{l}\text { Total xylose } \\
\text { consumed } \\
(\%)\end{array}$ & $\begin{array}{l}\text { Total glucose } \\
\text { consumed } \\
\text { (\%) }\end{array}$ \\
\hline XltA & $6.0 \times 10^{-3} \pm 1.7 \times 10^{-4}$ & $33.8 \pm 1.5$ & $5.5 \times 10^{-3} \pm 1.1 \times 10^{-4}$ & ND & $23.6 \pm 0.4$ \\
\hline XltB & $6.3 \times 10^{-3} \pm 1.0 \times 10^{-4}$ & $56.5 \pm 1.8$ & $6.2 \times 10^{-3} \pm 2.6 \times 10^{-4}$ & $34.8 \pm 0.6$ & $15.7 \pm 0.1$ \\
\hline XltC & $7.7 \times 10^{-3} \pm 3.6 \times 10^{-4}$ & $50.0 \pm 2.3$ & $1.8 \times 10^{-2} \pm 7.6 \times 10^{-4}$ & ND & $46.9 \pm 5.8$ \\
\hline Str1 & $3.2 \times 10^{-3} \pm 3.6 \times 10^{-5}$ & NQ & $4.8 \times 10^{-3} \pm 4.4 \times 10^{-5}$ & ND & ND \\
\hline Str2 & $6.8 \times 10^{-3} \pm 1.8 \times 10^{-5}$ & $50.6 \pm 1.1$ & $1.7 \times 10^{-2} \pm 3.8 \times 10^{-4}$ & ND & 100 \\
\hline Str3 & $1.4 \times 10^{-2} \pm 1.5 \times 10^{-4}$ & 100 & $1.8 \times 10^{-2} \pm 8.1 \times 10^{-5}$ & $28.4 \pm 0.3$ & 100 \\
\hline
\end{tabular}


As was already observed in the plate assays, all transformant strains were able to grow in the presence of xylose as a sole carbon source. When comparing sugar uptake with growth curves, differences could be observed between the different transformants. With exception of the Str1 strain, in the presence of xylose as a sole carbon source growth started immediately after inoculation. In concordance to what has been observed in the plate assay, growth of Str1 transformant strain was observed only after 10 days. Some xylose consumption was observed by HPLC analysis, but due to the long lag time and poor growth this could not be properly quantified. Together, these results suggest that the xylose transport capacity of Str 1 transporter is very low. In contrast, the Str3 transformant showed the fastest growth rate in the presence of the pentose, followed by XltC, Str2, $\mathrm{XltB}$ and XltA. At the end of the culturing period Str3 had consumed all xylose present in the media. Str2, XltC and XltB had used 50-57\% of the available xylose, while XltA used the $34 \%$ (Table 1). In the presence of the xylose and glucose mixture $(0.5+0.5 \% ; w / v)$, each of the transformant strains behaved differently.

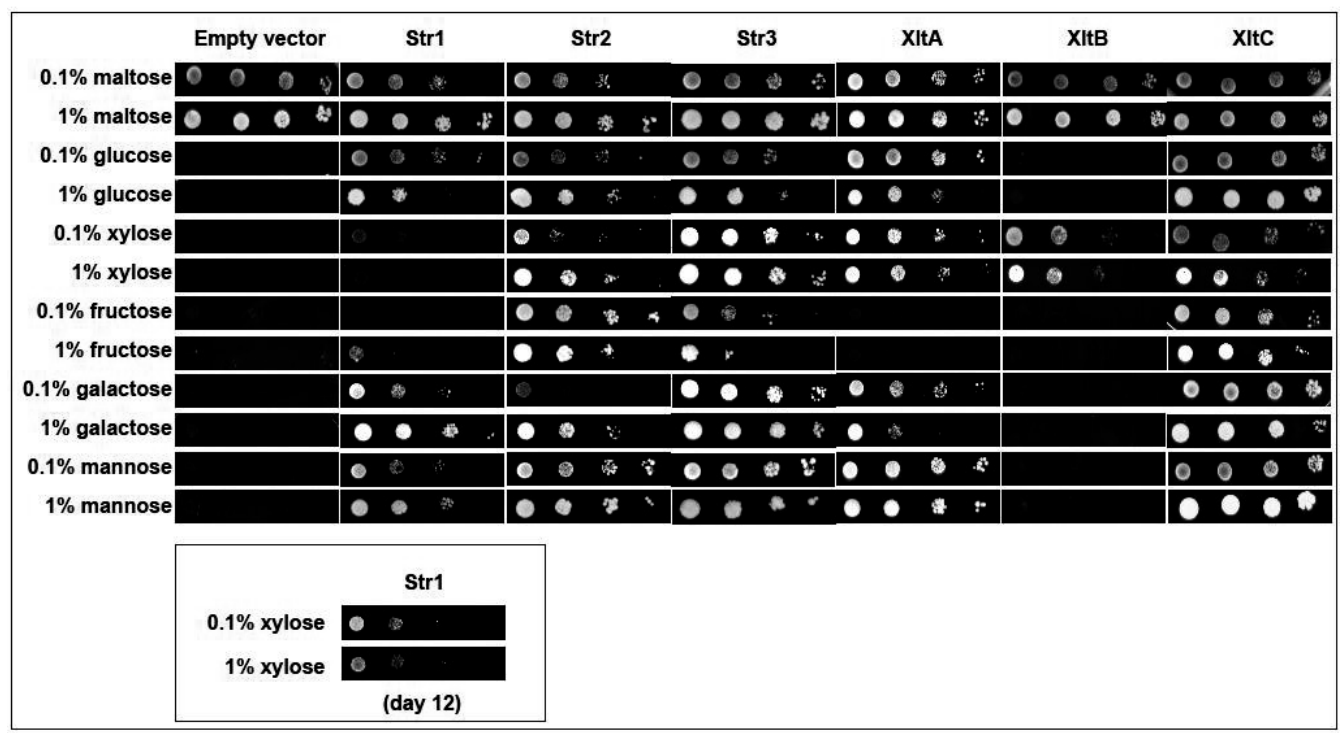

Figure 4 Heterologous expression and substrate utilization analysis of the A. niger sugar transporters XltA-C and T. reesei sugar transporters Str1-3. Ag11C3 transformants expressing str 1, str2, str $3, x l t A, x l t B$ or $x l t C$ were grown for 7 days at $30{ }^{\circ} \mathrm{C}$ in minimal medium agar plates containing a final concentration of $1 \%(\mathrm{w} / \mathrm{v})$ or $0.1 \%(\mathrm{w} / \mathrm{v})$ of the following sugars: maltose, glucose, xylose, fructose, galactose and mannose. EV: Ag11C3, carrying the empty expression vector p426HXT7-6His. Insert str 1 transformant grown for 12 days on xylose plates 
In comparison to xylose cultures, growth of the Str1, Str2, Str3 and XltC transformant strains was enhanced by the addition of glucose, whereas growth of the XltB transformant was similar, and the XltA transformant grew less. This can be explained by different affinities of the respective sugar porters toward glucose and xylose. Str 2 and Str 3 transformant strains were able to use glucose efficiently (Table 1). In the presence of glucose, no xylose uptake could be determined with the Str2 transformant, whereas the Str3 transformant was able to use both carbon sources simultaneously. Xylose uptake by the XltC transformant was inhibited by glucose as well, but utilization of the hexose was not as efficient as observed for Str2 and Str3 (Table 1). In the presence of glucose Str1 initially grew faster, suggesting that Str1 could also transport glucose in the presence of the pentose, but growth levels when reaching the stationary phase were lower than observed in the cultures with xylose. Again sugar utilization could not be properly monitored by HPLC analysis suggesting a very low rate of glucose uptake as well. When compared to the xylose medium, the growth rate of the Ag11C3 transformants expressing $A$. niger XltA and XltB was not higher in the mixed xylose-glucose medium, and in contrast to the $T$. reesei $\mathrm{Str} 2$ and $\mathrm{Str} 3$ transporters, glucose transport by $A$. niger XltA and XltB was not efficient. Moreover, the overall carbon uptake (glucose + xylose) in the sugar mix cultures was lower than that one observed for the xylose cultures (Table 1). This was also the case for the Ag11C3-XltC transformant. In the presence of glucose XltA was unable to transport xylose, while glucose was transported at a low rate (even lower than the xylose transport levels when the pentose was used as the sole carbon source). Inhibition of a xylose-specific transporter by glucose has been previously reported for the well-characterized XylE, from E. coli [13]. Even more remarkable was the performance of the XltB transformant in the mixed xyloseglucose medium: in the presence of glucose, xylose uptake was reduced but still two times higher than the uptake of glucose. Together with the plate assay study (Figure 4), these results strongly suggest that XltB is a transporter specific for xylose. To get more insights about both the biochemical properties and the biological role of these transporters, radiolabeled sugar uptake studies and transcriptional analysis of the respective coding genes were performed.

\section{Analysis of uptake kinetics by ${ }^{14} \mathrm{C}$-labeled sugar uptake studies}

The growth data presented above effectively demonstrated that all the candidate transporters are able to transport xylose. Differences observed in growth rate and sugar uptake of yeast transformants expressing individual transporters suggested important differences in substrate specificity and uptake rate. We therefore measured the kinetics of xylose and glucose uptake of the six transporters. 
Transport assays were performed using a range of D- $\left[1-{ }^{14} \mathrm{C}\right]$-xylose and D-[U$\left.{ }^{14} \mathrm{C}\right]$-glucose concentrations. Initial uptake rates were calculated, fitted to the Michaelis- Menten model and used to estimate the appropriate kinetic parameters $\left(\mathrm{K}_{\mathrm{m}}\right.$ and $\left.\mathrm{V}_{\max }\right)$ as previously described [40] (Table 2) (Additional file 7). The affinity for xylose of the six transporters ranged from $90 \mu \mathrm{M}$ (XltA) to $15 \mathrm{mM}$ (XltB), being, in most cases, higher than those reported for most of the fungal xylose transporters characterized to date, with values between $80 \mu \mathrm{M}$ and $150 \mathrm{mM}[23,24,26,38]$. A. niger XltA showed to have a very high affinity for xylose $(0.09 \pm 0.03 \mathrm{mM})$, which is even higher than reported for the $E$. coli xylose transporter XylE $(0.47 \mathrm{mM})[20,22]$, and the A. niger high-affinity sugar transporter MstA $(0.3 \pm 0.1 \mathrm{mM})[38]$. The transporters XltC $(4.71 \pm 1.04 \mathrm{mM})$, Str1 $(5.70 \pm 0.19 \mathrm{mM}), \operatorname{Str} 2(6.18 \pm 0.81 \mathrm{mM})$ and $\operatorname{Str} 3(2.19 \pm 0.29 \mathrm{mM})$ showed a high affinity toward xylose within the same order of magnitude, whereas XltB $(15.00 \pm 4.50 \mathrm{mM})$ had a slightly lower affinity. Regarding glucose transport characteristics, five out of six transporters showed a very high affinity for the hexose, ranging from $13 \mu \mathrm{M}$ (Str1) to $108 \mu \mathrm{M}$ (XltC).

Table 2 Xylose and glucose initial uptake kinetics of fungal MFS transporters

\begin{tabular}{|c|c|c|c|c|c|}
\hline \multirow[b]{2}{*}{ Transporter } & \multicolumn{2}{|c|}{ Xylose uptake kinetics } & \multicolumn{2}{|c|}{ Xylose uptake kinetics } & \multirow[t]{2}{*}{ References } \\
\hline & $\begin{array}{l}\text { Vmax }\left(\mathrm{nmol} \mathrm{min}^{-1}\right. \\
\left.\mathrm{mg} \mathrm{DW}^{-1}\right)\end{array}$ & $\mathrm{Km}(\mathrm{mM})$ & $\begin{array}{l}\text { Vmax (nmol min }{ }^{-1} \\
\left.\text { mg DW }^{-1}\right)\end{array}$ & $\mathrm{Km}(\mathrm{mM})$ & \\
\hline AnXltA & $1.08 \pm 0.05$ & $0.09 \pm 0.03$ & $1.11 \pm 0.15$ & $0.07 \pm 0.01$ & This study \\
\hline AnXltB & $0.10 \pm 0.00$ & $15.0 \pm 4.50$ & ND & ND & This study \\
\hline AnXltC & $0.14 \pm 0.01$ & $4.71 \pm 1.04$ & $1.18 \pm 0.15$ & $0.11 \pm 0.02$ & This study \\
\hline TrStr1 & $0.04 \pm 0.01$ & $5.70 \pm 0.19$ & $0.14 \pm 0.04$ & $0.01 \pm 0.00$ & This study \\
\hline TrStr2 & $0.12 \pm 0.02$ & $6.18 \pm 0.81$ & $0.69 \pm 0.19$ & $0.05 \pm 0.01$ & This study \\
\hline TrStr3 & $0.46 \pm 0.04$ & $2.19 \pm 0.29$ & $1.31 \pm 0.33$ & $0.06 \pm 0.01$ & This study \\
\hline AnMstA & $\mathrm{NC}$ & $0.3 \pm 0.1$ & $\mathrm{NC}$ & $0.03 \pm 0.01$ & [38] \\
\hline GXS1 & $0.01 \pm 0.00$ & $0.08 \pm 0.02$ & - & - & [17] \\
\hline XUT3 & $0.01 \pm 0.00$ & $4.09 \pm 1.08$ & - & - & [17] \\
\hline Хур29 & $0.61 \pm 0.05$ & $175.74 \pm 21.36$ & ND & ND & [26] \\
\hline An25 & $0.69 \pm 0.04$ & $55.96 \pm 9.37$ & ND & ND & [26] \\
\hline SUT1 & $132.0 \pm 1.0$ & $145.0 \pm 1.0$ & $45.0 \pm 1.0$ & $1.5 \pm 0.1$ & [23] \\
\hline \multirow[t]{2}{*}{ SUT2+ } & $41.0 \pm 1.0$ & $49.0 \pm 1.0$ & $3.3 \pm 0.1$ & $1.1 \pm 0.1$ & {$[23]$} \\
\hline & & & $28.0 \pm 4.0$ & $55 \pm 11.0$ & \\
\hline \multirow[t]{2}{*}{ SUT3+ } & $87.0 \pm 2.0$ & $103.0 \pm 3.0$ & $3.7 \pm 0.1$ & $0.8 \pm 0.1$ & [23] \\
\hline & & & $22.0 \pm 0.1$ & $31.0 \pm 0.1$ & \\
\hline Ag11C3 & ND & ND & ND & ND & This study \\
\hline
\end{tabular}


These are, in all cases, in the same range as reported for other $A$. niger highaffinity glucose transporters [9, 38]. It is a common feature of reported glucose/xylose transporters to show higher affinity for the hexose than for the pentose [26], with differences of around two orders of magnitude [20]. This was observed for the three $T$. reesei transporters characterized in this study, while the $A$. niger XltC affinity toward glucose was around 50 times higher than toward xylose (Table 2). However, A. niger XltA showed approximately the same high affinity for xylose $(0.09 \pm 0.03 \mathrm{mM})$ as for glucose $(0.07 \pm 0.01 \mathrm{mM})$. Also, XltA was able to transport both sugars at the same rate. In contrast, uptake of radiolabeled glucose by XltB was not detected, as was previously described for the $P$. stipitis Xyp29 and the $N$. crassa An25 xylose transporters [26]. This result was in concordance with the inability of XltB to support the growth of the Ag11C3 strain in the presence of glucose as a sole carbon source. The biochemical characteristics of the six studied transporters, plus those reported previously $[9,37,38]$, revealed the wide range of glucose and xylose uptake systems featured by these fungi. The initial uptake kinetics of both sugars displayed by XltA and XltB suggested a possible biological role for these transporters in xylose utilization.

\section{Transcriptional analysis of $T$. reesei str $1-3$ and $A$. niger xlt $A-C$}

To shed more light about their possible biological role on xylose uptake, a transcriptional analysis, performed via RT-qPCR of the respective transporter coding genes, was done. For this, the expression of A. niger xltA, xltB and $x l t C$; and $T$. reesei strl, str 2 and str 3 was studied, in different culture conditions, in both wild-type and $x \operatorname{lnR} / x y r 1$ mutant strains which harbor an inactivated transcriptional activator of the xylanolytic system (Figure 5). The samples for expression analysis of the $A$. niger genes were obtained from mycelium grown in cultures containing minimal medium plus $55 \mathrm{mM}$ glucose, $66 \mathrm{mM}$ xylose, 0.5 $\mathrm{mM}$ xylose, or no carbon source (NCS), whereas T. reesei samples were isolated from cultures on minimal medium containing one of the following carbon sources: $55 \mathrm{mM}$ glucose, $66 \mathrm{mM}$ xylose, $1.5 \mathrm{mM}$ sophorose, or NCS. In case of the $A$. niger transporter genes xltA was strongly induced by high xylose concentrations, and its expression levels were also significantly higher in lower xylose concentrations when compared to the rest of the conditions studied (Figure 5). XlnR is a transcriptional activator of the xylanolytic system in $A$. niger [41]. In the $x \ln R$ mutant strain, $x l t A$ transcript levels dropped dramatically in the presence of low and high xylose concentrations, while they were kept at the same level in the NCS and glucose conditions. This indicates a clear role of $\mathrm{X} \operatorname{lnR}$ in the transcriptional regulation of $x l t A$, and thus, a role of XltA in xylose 
uptake by $A$. niger. The xltB gene expression was apparently not xylose dependent as it was repressed in the presence of high concentrations of both glucose and xylose, and its expression levels in the presence of low xylose concentrations were slightly lower than in the NCS condition. Also, the expression profile of $x l t B$ was similar in the wild-type and the $\Delta x \ln R$ strain. Therefore, a role of $X \ln R$ in the regulation of this gene in the studied conditions could be discarded. According to $x l t B$ expression profile, XtlB seems to be relevant in $A$. niger when there is a low availability of carbon sources in the environment. In the uptake kinetics studies, the transport of low xylose concentrations by XltB could be determined, but it was not possible for low glucose concentrations. Thus, the substrate specificity, and the higher affinity for xylose than for glucose shown by XltB, together with its preferential expression at low carbon source concentrations, indicates that this transporter could have a role for xylose transport in the fungus independent of XlnR activation. The expression levels of $x$ lt $C$ were, in contrast, higher when high sugar concentrations were present in the medium. They were particularly high in the xylose $66 \mathrm{mM}$ condition. The role of XlnR on the transcriptional regulation of this gene was not clear, as its expression levels in the mutant strain were only slightly reduced in the xylose $0.5 \mathrm{mM}$ condition, but not reduced at all in the xylose $66 \mathrm{mM}$ condition. Regarding the $T$. reesei genes, strl expression was strongly induced by xylose, in a similar way to A. niger xltA, and also by sophorose, which is also an inducer of the $T$. reesei xylanolytic system [42]. In addition, it was clearly transcriptionally regulated by Xyr1, encoding the main regulator of the xylose metabolism in T. reesei [43]. Despite Str1 low efficiency in xylose transport, the transcriptional behavior of its coding gene, and the recent findings reported by Huang et al. [28], suggests an important role for the transporter in xylose utilization. In the wild-type and $\Delta x y r 1$ strains, str 2 was preferentially expressed in resting cell conditions and strongly down-regulated in the presence of $55 \mathrm{mM}$ glucose. In case of the xylose and sophorose conditions, str2 expression levels were slightly higher in the $x y r l$ deletion strain. The str 3 gene did not seem to be specifically induced by xylose, being its expression levels higher in the presence of glucose (Figure 5). In the absence of Xyr1 ( $\triangle x y r 1$ strain), str3 expression levels dropped in the presence of xylose and sophorose, but the same was observed in the glucose condition, while its expression was slightly increased when no carbon source was present. Although a role of Xyr1 in str3 regulation cannot be discarded, the obtained results are not conclusive enough. The higher str 3 expression levels observed in the presence of $66 \mathrm{mM}$ xylose and especially in $55 \mathrm{mM}$ glucose, when compared to low concentrations of sophorose and the NCS condition (wild-type strain), do suggest a role for Str3 in the uptake of high 
sugar concentrations. This hypothesis is also in agreement with the glucose and xylose transporting behavior shown by the Str3-expressing yeast strain constructed in this study.

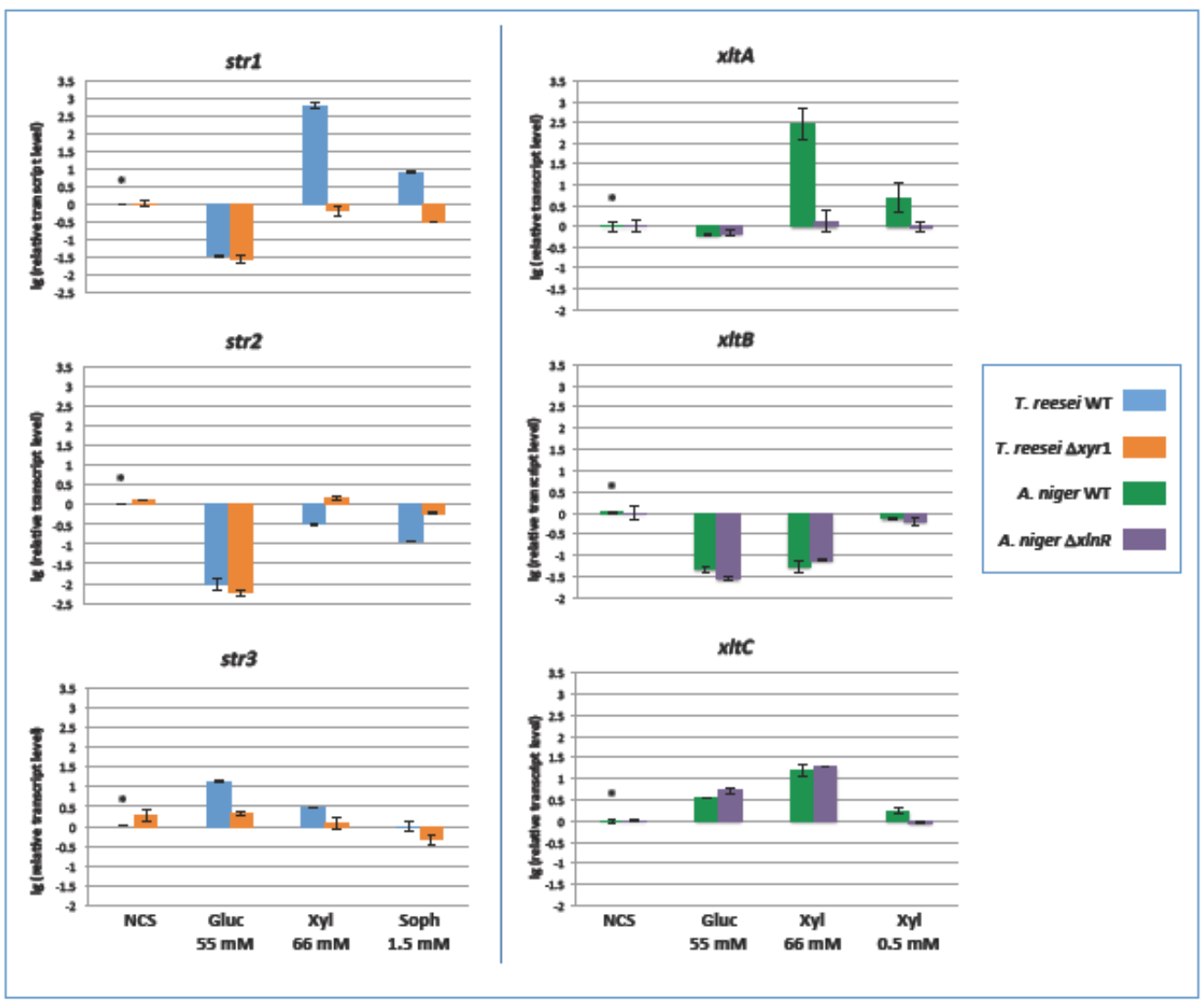

Figure 5 Transcriptional analysis of T. reesei str 1-3 and A. niger xltA-C. Samples were taken $3 \mathrm{~h}$ after mycelium transfer to the different culture conditions, and expression analyses were performed by RT-qPCR. Normalization of the expression data was done using the histone-like gene "hist" transcript (gene ID 207921) for A. niger, and genes sar 1 and act1 for T. reesei. Results are given as relative transcript ratios in logarithmic scale (lg). The values provided in the figures are means of two biological replicates. Transcript levels always refer to the reference sample, indicated with an asterisk 


\section{Characteristics defining AnXItA-C and TrStr1-3 xylose transporters}

Xylose was a substrate for all six different transporters, suggesting that the HMMxylT may have captured residues discriminating for glucose-xylose porters. With the aim of pinpointing these discriminating residues, that should be conserved in the novel xylose transporter proteins, we used an MSA of all transporters used for $\mathrm{HMM}_{\mathrm{gluT}}$ (constructed in a previous study, [9]) and HMMxylT (this study), plus the newly identified $A$. niger and $T$. reesei transporters (Additional file 8). A number of motifs and residues from fungal sugar porters have been recently reported to be relevant for xylose transport by different studies, and the amino acid sequences of AnXltAC and TrStr1-3 were analyzed for the presence of these motifs (Figure 6). Wang and collaborators suggested the relevance of the aromatic residue enriched motif YFFYY (332-336), present in the transmembrane section 7, for the xylose transport capacity of Mgt05196p from Meyerozyma guilliermondii [44]. TrStr3, which showed the highest xylose transport capacity in this study, also contains the YFFYY (320-324) peptide. The same motif is present as well in a number of sugar porters (MSA), but it is not completely conserved in transporters that are exclusive for xylose as XylE from E. coli, An25 from N. crassa or Xyp29 (also called Xut6 and Stl12) from P. stipitis : the first three aromatic residues from the YFFYY motif are substituted by aliphatic ones in XylE and An25. This was also found to be the case in AnXtlB (ALIYY); AnXltC (VMMYY) (although not strictly an aliphatic residue, methionine is usually considered as such, since its sulfur group is not reactive); TrStr1 (AVLYY); and TrStr2 (ALIYY).

\begin{tabular}{|c|c|c|c|}
\hline C. intermedia_GXS1 & FAASGGVLFGYDTGTISGVMTMD 54 & LLCAGRVIAGFG 137 & QAFQQLTGVNFIFYYGTTFFKRAGV 313 \\
\hline M. guilliermondii_Mgt05196 & LVAFGGFVFGFDTGTISGFVNMT 83 & QIVIGRAIAGLA 170 & QSMQQLTGNNYFFYYGTTIFKAVGM 346 \\
\hline A. niger_XItA & FASLGGFVYGYNQGMFSEILTMN 61 & YVFAGRFVTGLG 142 & MLFQQWNGINAINYYAPQVFEGLEL 343 \\
\hline A. niger_XItB & CSTLGGLTFGYDQGVVSVILVMD 81 & MLTVARLIGGVG 160 & MFFQQFVGINALIYYAPTLFETMGL 353 \\
\hline A. niger_XItC & IAVVGGGLFGFDISSMSAIIETD 34 & MLIVGRIINGLS 127 & QIWSQLTGMNVMMYYITYVFAMAGL 302 \\
\hline T. reesei_Str1 & FACIGGVLYGYNQGMFSGVLAMP 65 & AILGGRFVTGMG 152 & MFFQQWTGINAVLYYAPTIFQQLGQ 353 \\
\hline T. reesei_Str2 & FASLGGFLFGYDQGVVSGVLGME 55 & MLFGGRAVAGLA 135 & MFLQQFQGCNALIYYAPTIFGQLGL 357 \\
\hline T. reesei_Str3 & LVSMGGLIFGYDTGQISGFLEMP 67 & QVMIGRFVSGLG 154 & QMFQQLTGANYFFYYGTTIFKSVQI 334 \\
\hline N. crassa_An25 & VATTGFLLFGYDQGVMSGIITAP 43 & QLFVGRVVMGIG 127 & QFMQQVGGCNAVIYYFPILFQDSIG 306 \\
\hline P. stipitis_Xур29 & FAGVGFLLLGYDQGVMGSLLTLP 65 & HLTVARIITGLG 147 & QIMQQITGINIITYYAGTIFESYIG 330 \\
\hline P. stipitis_Hxt2.6 & VLTLTSTNNGYDGSMLNGLQSLS 74 & FFFVSRMVIGFG 152 & ACMTQLSGNGLVSYYLSKVLISIGI 331 \\
\hline E. coli_XylE & $\begin{array}{c}\text { VATLGGLLFGYDTAVISGTVESL } 38 \\
\star:: \cdot \cdot\end{array}$ & $\begin{array}{c}\text { EFVIYRIIGGIG } 139 \\
*:^{\star} \ldots\end{array}$ & $\underset{*}{\operatorname{SIFQQFVGINVVLYYAPEVFKTLGA~}} 308$ \\
\hline
\end{tabular}

Figure 6 Conservation of motifs and residues reported to be relevant for xylose transport. A multiple sequence alignment of known xylose transporters, including A. niger XltA-C and T. reesei Str1-3 was constructed using the EMBL-EBI Clustal Omega tool [68]. Reported relevant motifs and residues are in bold; amino acids highlighted in blue indicate the transporter where motifs/residues were described to be relevant for xylose transport 
In addition, the same motif in AnXltA (AINYY) had a polar residue (N) at the third position. Although most of the functionally validated sugar transporters contain a nonpolar (aromatic or aliphatic) amino acid at that position, several sugar porters, including the xylose transporters Hxt2.6 (LVSYY) and Xyp 29 (IITYY) from P. stipitis, contain a polar residue as well. The Mgt05196p residues aspartate D72 and arginine R164, also suggested to be crucial for xylose transport [44], were found to be conserved in AnXltB, AnXltC, TrStr2 and TrStr3. AnXltA and $\operatorname{TrStr} 1$ had the arginine conserved, but both contained an asparagine residue at the aspartate 72 of Mgt05196p. Since asparagine and aspartate are similar amino acids, both might have the same function at that particular position. The motif GG/FXXXG, present at the first transmembrane span of sugar porters and highly enriched in those that confer growth on xylose [18], was also found to be present in AnXltA-C and TrStr1-3 (Figure 6). Knoshaugh and collaborators [45] recently highlighted the variability of this motif, that allow to distinguish between xylose transporters (GGLXXGYD/N), arabinose transporters (XGXXFGFD) and glucose transporters (GGFXFGWG). This motif was subjected to protein engineering through saturation mutagenesis in the $C$. intermedia glucose-xylose symporter 1 (GXS1) [18]. In GXS1, the wild-type motif is GGVLFG (36-41). Saturation mutagenesis for each of the three variable residues (V38, L39, F40) produced changes in selectivity and efficiency of monosaccharide transport by GXS1. Residues V38 and F40 were found to be involved in carbon source selectivity, and L39 in controlling substrate transport efficiency. Interestingly, some of the most significant V38 substitutions were also found in the here studied transporters. V38F, that almost completely attenuated glucose exponential growth rate while amplifying exponential xylose growth rate by $50 \%$, was found in XltA and Str2. V38L, that increased the exponential xylose growth rate by 73 $\%$ without altering glucose exponential growth rate significantly, was found in XltB and Str3. V38G, which also produced a positive effect on xylose transport, was found in XltC. The L39 substitutions resulted in a general or differential attenuation of GXS1 transport function. L39I, found to be responsible for a specific attenuation of glucose transport in favor of xylose transport, was found in Str3. The L39V substitution, present in all three P. stipitis high-velocity xylose transporters SUT1-3 [23], was also found in XltA. Regarding the GXS1 F40 residue, XltB, XltC, Str2 and Str3 have the same amino acid at the same position, whereas XltA and Str1 contain a Y. The F40Y substitution in GXS1 produced an attenuating effect on the transport of xylose and other monosaccharides, indicating that the particular tyrosine residue could have the same role in XltA and Str1. This could explain, in part, the low efficiency displayed by Str1 on xylose transport, and provides a hint on how the transport capacity of both 
transporters could be improved. Regarding the presence of amino acids that have been shown to be key at certain positions for glucose affinity in yeast sugar porters, like the threonine 219/213 and the asparagine 376/370 of S cerevisiae Gal2/Hxt7 [20, 46], interesting features were also found. Only XltC, Str2 and Str3, that were (Table 1), keep both residues conserved; while XltB and Str1, that showed the lowest glucose transport capacity, have different residues in both positions. In summary, the amino acid sequence analysis of the six transporters showed that all of them harbor motifs and residues previously associated to fungal xylose transporters. This fact, in combination with the experimental evidences provided, indicates a role of these transporters in xylose uptake.

\section{Conclusions}

In this study, computational and experimental approaches were successfully combined for the identification and characterization of xylose transporting proteins from the industrial cell factories $A$. niger and $T$. reesei. Comparing the HMM xylт output with recently published transcriptome studies, also taking into account phylogenetic distance relationships, was a good strategy to link a specific group of MFS porters with the utilization of lignocellulosic feedstocks. Using the mentioned methodology, five putative xylose transporters (XltA, XltB, XltC, Str2, Str3), and the recently identified xylose transporter Str1, were selected and successfully validated as xylose transporters. All of them displayed significant differences in their substrate specificity and biochemical properties, being XltA and XltB of particular interest, due to the high affinity for xylose of the former, and the narrow substrate specificity of the latter. Also, new insights about the regulation, at transcriptional level, of xylose utilization by A. niger and T. reesei were found, the most remarkable being that xylose uptake is not completely controlled by the $\mathrm{X} \operatorname{lnR} / \mathrm{Xyr} 1$ regulon. To the best of our knowledge, this is the first study on the functional validation and characterization of sugar porters with their biological role specifically associated to xylose transport in A.niger. Also, the biochemical characterization of xylose transporters in T. reese $i$ is reported for the first time. In summary, this study contributes to a better understanding of xylose utilization by two relevant industrial filamentous fungi, and provides new tools for strain engineering in fungi. 


\section{Methods}

\section{Construction of a xylose hidden Markov model}

The protein sequences used to build $\mathrm{HMM}_{\mathrm{xylT}}$ were obtained from the UniProt database [47], and aligned using the PRALINE structural alignment tool [48] with the same parameters as described for the hidden Markov model constructed in our previous work [9]. HMMxylT was built using the HMMER v3.0 tool [49]. The A. niger ATCC1015 [50] and the T. reesei Rut-C30 [51] proteomes, which were used for the in silico analysis, were downloaded from the JGI database [52].

\section{Strains and growth conditions}

Escherichia coli DH5a [endA1, hsdR17, gyrA96, thi-1, relA1, supE44, recA1, 4 lacU169 (\$80 lacZ4M15)] was used for cloning experiments and plasmid propagation. It was grown at $37{ }^{\circ} \mathrm{C}$ on an LB medium ( $1 \%$ tryptone, $0.5 \%$ yeast extract, $1 \% \mathrm{NaCl} ; w / v$ ), with $100 \mu \mathrm{g} \mathrm{mL}-1$ ampicillin when required for transformants selection. A. niger N400 (CBS 120.49), NW199 (fwnA6, leuA5, goxC17, pyrA6; $\triangle x \ln R:: p I M 240$ ) [53] and T. reesei QM6aAtmus53 (ATCC 13631) [54] and QM6aAtmus534xyrl (ATCC 13631) [55] strains were used in mycelium transfer experiments for the MFS genes transcriptional analysis. The $A$. niger and $T$. reesei strains were maintained on complete medium agar [56] and malt extract agar (MEX), respectively, at $30{ }^{\circ} \mathrm{C}$. Mycelium transfer experiments of both fungal species were performed in a similar way, using liquid cultures in Erlenmeyer flasks on a rotary shaker. The A. niger strains were pre-cultured at $30{ }^{\circ} \mathrm{C}$ and $200 \mathrm{rpm}$, during $18 \mathrm{~h}$, in minimal medium containing $4.50 \mathrm{~g} \mathrm{~L}^{-1}$ $\mathrm{NaNO}_{3}, 1.13 \mathrm{~g} \mathrm{~L}-1 \mathrm{KH}_{2} \mathrm{PO}_{4}, 0.38 \mathrm{~g} \mathrm{~L}-1 \mathrm{KCl}, 0.38 \mathrm{~g} \mathrm{~L}-1 \mathrm{MgSO}_{4} \cdot 7 \mathrm{H}_{2} \mathrm{O}, 750 \mu \mathrm{L} \mathrm{L}-1$ of Vishniac solution, and $100 \mathrm{mM}$ sorbitol $[56,57]$. Equal amounts of waterrinsed mycelium were transferred to minimal medium with the following carbon source compositions: $55 \mathrm{mM}$ d-glucose, $66 \mathrm{mM}$ d-xylose, $0.5 \mathrm{mM}$ d-xylose or no carbon source (NCS). The initial $\mathrm{pH}$ of the medium in all conditions was set at 6.0. The T. reesei strains were pre-cultured in Mandels-Andreotti (MA) medium [58], containing $1 \%(w / v)$ glycerol as the sole carbon source, at $30{ }^{\circ} \mathrm{C}$ and $180 \mathrm{rpm}$ for $22 \mathrm{~h}$. Pre-grown mycelia were washed, then equal amounts were resuspended in MA media containing $55 \mathrm{mM}$ d-glucose, $66 \mathrm{mM}$ d-xylose, 1.5 $\mathrm{mM}$ sophorose or in medium without carbon source (NCS). In both experiments, $3 \mathrm{~h}$ after mycelium transfer samples were taken and quickly washed, dried with a single-use towel, snap-frozen with liquid nitrogen and stored at $-80{ }^{\circ} \mathrm{C}$ until further processing. Two biological replicates per condition were studied in all cases. The $S$. cerevisiae strain EBY.VW4000 (CEN.PK2-1C hxt134:: loxP;

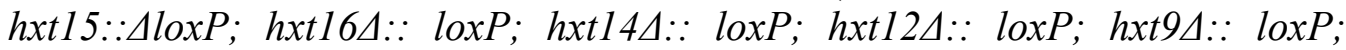


hxt114:: loxP; hxt104:: loxP; hxt84:: loxP; hxt5144:: loxP; hxt $24: \because \operatorname{loxP}$;

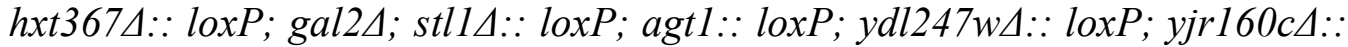
lox $P$ ) [10], previously transformed with the plasmid pRH315 [39], expressing the d-xylose reductase and xylitol dehydrogenase from $P$. stipitis, and the $S$. cerevisiae xylulokinase, was used as a xylose-utilizing strain for the characterization of sugar transporters. It was grown at $30{ }^{\circ} \mathrm{C}$ and maintained in the solid complete medium containing $10 \mathrm{~g} \mathrm{~L}_{-1}$ of yeast extract, $20 \mathrm{~g} \mathrm{~L}_{-1}$ of peptone and $20 \mathrm{~g} \mathrm{~L}-1$ of maltose. The EBY. VW4000-derived strains obtained in the present study were grown in the liquid minimal medium containing $6.7 \mathrm{~g} \mathrm{~L}-1$ of yeast nitrogen base with ammonium sulfate (Difco), $20 \mathrm{~g} \mathrm{~L}-1$ of maltose, supplemented with leucine (30 mg L-1) and histidine (20 mg L-1). Growth rates $(\mu)$ of the Ag11C3 transformants, during time course cultivations, were calculated using the O.D. values obtained from $T=0$ until the cultures reached stationary phase.

\section{Construction of $S$. cerevisiae Ag11C3 transformants expressing A. niger and} T. reesei genes

The coding sequence of the genes $x l t A, x l t B, x l t C$, str $1, s t r 2$ and str 3 was obtained through PCR amplification of $A$. niger and $T$. reesei cDNA samples, respectively. The coding sequence of the gene $x l t A$, digested with SpeI and XhoI was cloned on the S. cerevisiae expression vector p426HXT7-6His [59], previously linearized with SpeI and XhoI, under the control of the constitutive promoter $\mathrm{HXT}_{\mathrm{p}}$ and the terminator $\mathrm{CYCl}_{\mathrm{t}}$. The $x l t B, x l t C$, strl, str2 and str $3 \mathrm{cDNA}$ sequences were amplified with oligonucleotides containing 40 additional base pairs, corresponding to the p426HXT7-6His cloning site, and were cloned to the vector through yeast-mediated recombination [60]. Primer sequences and plasmids used in this study are provided in Additional file 9. The Ag11C3 yeast strain transformation was performed as previously described [61]. The curing of the p426HXT7-6His- $x l t A$ plasmid, containing the URA3 selection marker, from the Ag11 strain was done by growing the transformant in minimal medium plates containing maltose and uracil, thereby relieving the plasmid selective pressure. After three replating rounds in maltose-uracil plates, p426HXT7-6His-xltA cured strains were isolated through their growth on minimal medium plates containing maltose, uracil and 5-fluoroorotic acid (FOA). FOA is a commonly used agent to select for the absence of the URA3 gene in yeast strains [62]. A single colony growing on the FOA plate, named $\mathrm{Ag} 11 \mathrm{C} 3$, was selected for its use as host for the expression of new xylose transporter candidates. 


\section{Sugar analyzes}

Xylose and glucose present in the $S$. cerevisiae culture supernatants were quantified by high-pressure liquid chromatography (HPLC) analysis. The samples were centrifuged at maximum speed in a benchtop centrifuge for $10 \mathrm{~min}$ and analyzed on a Dionex ICS-5000+ instrument (Thermo Scientific), equipped with a CarboPac MA1 column. Separation was performed by isocratic elution with $480 \mathrm{mM} \mathrm{NaOH}$, at a flow rate of $0.4 \mathrm{~mL}$ min-1 for $35 \mathrm{~min}$.

\section{Analysis of uptake kinetics by ${ }^{14} \mathbf{C}$-labeled sugars' uptake studies}

Sugars' uptake assays were performed as described [63], with minor adjustments. A pre-inoculum $(50 \mathrm{~mL})$ of Synthetic Enhanced medium (SE; $6.7 \%(w / v)$ YNB w/o amino acids (Difco) $+20 \mathrm{mg} \mathrm{L}-1$ l-arginine and l-methionine), containing appropriate amino acids, and $2 \%(w / v)$ maltose as a carbon source, was inoculated and incubated for $48 \mathrm{~h}\left(30^{\circ} \mathrm{C}, 225 \mathrm{rpm}\right)$. The pre-inoculum was then transferred to $200 \mathrm{~mL}$ of fresh SE-medium, and after 48-h incubation, transferred to $500 \mathrm{~mL}$ fresh SE-medium. After 24-h incubation, cells were harvested by centrifugation $(4000 \mathrm{~g} ; 10 \mathrm{~min}$ ) and washed with $50 \mathrm{~mL}$ ice-cold ultrapure water. Cells were then washed and resuspended in ice-cold $100 \mathrm{mM}$ phosphate-buffered saline (PBS), $\mathrm{pH} 6.5$, to an $\mathrm{OD}_{600}$ of approximately 500, divided in $40-\mu \mathrm{L}$ aliquots, and kept on ice. Aliquots were incubated for $5 \mathrm{~min}$ at $30^{\circ} \mathrm{C}$ in a heat block with vigorous shaking before uptake assay was started. To start the reaction, $10 \mu \mathrm{L}$ of a 5 times concentrated D-[1- $\left.{ }^{14} \mathrm{C}\right]$-xylose or D-[U- $\left.{ }^{14} \mathrm{C}\right]$-glucose solution (Campro Scientific) was added. After exactly $20 \mathrm{~s}$, the reaction was stopped by the addition of $1 \mathrm{~mL}$ of appropriate ice-cold quenching buffer (100 $\mathrm{mM}$ PBS, pH 6.5, with $500 \mathrm{mM}$ unlabeled d-xylose or d-glucose), followed by vacuum filtration $(0.45 \mu \mathrm{m}$ HV filters, 1225 sampling manifold, Millipore), and two subsequent washing steps with $5 \mathrm{~mL}$ of ice-cold quenching buffer. After 5 min of drying in the vacuum manifold, the filters were transferred to scintillation vials with $4 \mathrm{~mL}$ scintillation liquid (Ultima Gold, Perkin Elmer), and activity was counted (Packard Tricarb 1600TR). All reactions were performed in triplicates. All values were corrected using triplicate negative control measurements without incubation, where the quenching solution was added prior to the addition of labeled substrate. Uptake rates at two typical substrate concentration ranges were measured; $1-100 \mu \mathrm{M}$ and $0.1-40 \mathrm{mM}$. Substrate solutions with an activity of approximately 5-5000 Bq $\mu \mathrm{L}-1$ were used. To determine kinetic parameters $\mathrm{K}_{\mathrm{m}}$ and $\mathrm{V}_{\max }$, the data were fitted to the Michaelis-Menten model using the least squares

$$
V=\frac{V_{\text {max }} \cdot[S]}{K_{m}+[S]}
$$

method. 
Transcriptional analysis of $A$. niger and $T$, reesei genes

RNA isolation from A. niger [9] and T. reesei [42] mycelium was done as described previously. Reverse transcription, quantitative PCRs and calculations were performed following the protocols and instruments described in MachAigner et al. [64]. Primer sequences are provided in Additional file 9. Cycling conditions and control reactions were performed as described previously [65]. Normalization of the expression data was done using the previously described histone-like gene "hist" transcript (gene ID 207921) [64] for A. niger, and genes sarl and actl for $T$. reesei [65]. 


\section{References}

1. Magnuson JK, Lasure LL. Organic acid production by filamentous fungi. New York: Springer; 2004. p. 307-40.

2. Jang Y-S, Kim B, Shin JH, Choi YJ, Choi S, Song CW, Lee J, Park HG, Lee SY. Bio-based production of C2-C6 platform chemicals. Biotechnol Bioeng. 2012;109:2437-59.

3. Punt PJ, van Biezen N, Conesa A, Albers A, Mangnus J, van den Hondel C. Filamentous fungi as cell factories for heterologous protein production. Trends Biotechnol. 2002;20:200-6.

4. van den Brink J, de Vries RP. Fungal enzyme sets for plant polysaccharide degradation. Appl Microbiol Biotechnol. 2011;91:1477-92.

5. de Oliveira JMPF, de Graaff LH. Proteomics of industrial fungi: trends and insights for biotechnology. Appl Microbiol Biotechnol. 2011;89:225-37. 6. Lubertozzi D, Keasling JD. Developing Aspergillus as a host for heterologous expression. Biotechnol Adv. 2009;27:53-75.

7. Leandro MJ, Fonseca C, Gonçalves P. Hexose and pentose transport in ascomycetous yeasts: an overview. FEMS Yeast Res. 2009;9:511-25.

8. Punta M, Coggill PC, Eberhardt RY, Mistry J, Tate J, Boursnell C, Pang N, Forslund K, Ceric G, Clements J, Heger A, Holm L, Sonnhammer ELL, Eddy SR, Bateman A, Finn RD. The Pfam protein families database. Nucleic Acids Res. 2012;40(Database issue):D290-301.

9. Sloothaak J, Odoni DI, de Graaff LH, Dos Santos VAPM, Schaap PJ, Tamayo- Ramos JA. Aspergillus niger membrane-associated proteome analysis for the identification of glucose transporters. Biotechnol Biofuels. 2015;8:150. 10. Wieczorke R, Krampe S, Weierstall T, Freidel K, Hollenberg CP, Boles E. Concurrent knock-out of at least 20 transporter genes is required to block uptake of hexoses in Saccharomyces cerevisiae. FEBS Lett. 1999;464:123-8. 11. Coons DM, Vagnoli P, Bisson LF. The C-terminal domain of Snf3p is sufficient to complement the growth defect of snf3 null mutations in Saccharomyces cerevisiae: SNF3 functions in glucose recognition. Yeast. 1997;13:9-20.

12. Ozcan S, Dover J, Rosenwald AG, Wölfl S, Johnston M. Two glucose transporters in Saccharomyces cerevisiae are glucose sensors that generate a signal for induction of gene expression. Proc Natl Acad Sci USA. 1996;93:12428-32.

13. Sun L, Zeng X, Yan C, Sun X, Gong X, Rao Y, Yan N. Crystal structure of a bacterial homologue of glucose transporters GLUT1-4. Nature.

2012;490:361-6. 
14. Madej MG, Kaback HR. Evolutionary mix-and-match with MFS transporters II. Proc Natl Acad Sci USA. 2013;110:E4831-8.

15. Guan L, Kaback HR. Lessons from lactose permease. Annu Rev Biophys Biomol Struct. 2006;35:67-91.

16. Quistgaard EM, Löw C, Moberg P, Trésaugues L, Nordlund P. Structural basis for substrate transport in the GLUT-homology family of monosaccharide transporters. Nat Struct Mol Biol. 2013;20:766-8.

17. Young EM, Comer AD, Huang H, Alper HS. A molecular transporter engineering approach to improving xylose catabolism in Saccharomyces cerevisiae. Metab Eng. 2012;14:401-11.

18. Young EM, Tong A, Bui H, Spofford C, Alper HS. Rewiring yeast sugar transporter preference through modifying a conserved protein motif. Proc Natl Acad Sci USA. 2014;111:131-6.

19. Nijland JG, Shin H, de Jong RM, de Waal PP, Klaassen P, Driessen A.

Engineering of an endogenous hexose transporter into a specific d-xylose transporter facilitates glucose-xylose co-consumption in Saccharomyces cerevisiae. Biotechnol Biofuels. 2014;7:168.

20. Farwick A, Bruder S, Schadeweg V, Oreb M, Boles E. Engineering of yeast hexose transporters to transport d-xylose without inhibition by d-glucose. Proc Natl Acad Sci USA. 2014;111:5159-64.

21. Reznicek O, Facey SJ, de Waal PP, Teunissen AWRH, de Bont JAM, Nijland JG, Driessen AJM, Hauer B. Improved xylose uptake in

Saccharomyces cerevisiae due to directed evolution of galactose permease Gal2 for sugar co-consumption. J Appl Microbiol. 2015;119:99-111.

22. Davis EO, Henderson PJ. The cloning and DNA sequence of the gene xylE for xylose-proton symport in Escherichia coli K12. J Biol Chem. 1987;262:13928-32.

23. Weierstall T, Hollenberg CP, Boles E. Cloning and characterization of three genes (SUT1-3) encoding glucose transporters of the yeast Pichia stipitis. Mol Microbiol. 1999;31:871-83.

24. Leandro MJ, Gonçalves P, Spencer-Martins I. Two glucose/xylose transporter genes from the yeast Candida intermedia: first molecular characterization of a yeast xylose-H+ symporter. Biochem J. 2006;395:543-9. 25. Saloheimo A, Rauta J, Stasyk OV, Sibirny AA, Penttilä M, Ruohonen L. Xylose transport studies with xylose-utilizing Saccharomyces cerevisiae strains expressing heterologous and homologous permeases. Appl Microbiol Biotechnol. 2007;74:1041-52. 
26. Du J, Li S, Zhao H. Discovery and characterization of novel d-xylosespecific transporters from Neurospora crassa and Pichia stipitis. Mol BioSyst. 2010;6:2150-6.

27. Colabardini AC, Nicolas L, Ries A, Brown NA, Fernanda T, Savoldi M, Goldman MHS, Menino JF, Rodrigues F, Goldman GH, Ries LNA, Dos Reis TF. Functional characterization of a xylose transporter in Aspergillus nidulans. Biotechnol Biofuels. 2014;7:46.

28. Huang Z-B, Chen X-Z, Qin L-N, Wu H-Q, Su X-Y, Dong Z-Y. A novel major facilitator transporter TrSTR1 is essential for pentose utilization and involved in xylanase induction in Trichoderma reesei. Biochem Biophys Res Commun. 2015;460:663-9.

29. van der Veen D, Oliveira JM, van den Berg WAM, de Graaff LH. Analysis of variance components reveals the contribution of sample processing to transcript variation. Appl Environ Microbiol. 2009;75:2414-22. 30. de Souza WR, de Gouvea PF, Savoldi M, Malavazi I, de Souza Bernardes LA, Goldman MHS, de Vries RP, de Castro Oliveira JV, Goldman GH. Transcriptome analysis of Aspergillus niger grown on sugarcane bagasse. Biotechnol Biofuels. 2011;4:40.

31. Delmas S, Pullan ST, Gaddipati S, Kokolski M, Malla S, Blythe MJ, Ibbett R, Campbell M, Liddell S, Aboobaker A, Tucker GA, Archer DB. Uncovering the genome-wide transcriptional responses of the filamentous fungus Aspergillus niger to lignocellulose using RNA sequencing. PLoS Genet. 2012;8:e1002875.

32. De Souza WR, Maitan-Alfenas GP, de Gouvêa PF, Brown NA, Savoldi M, Battaglia E, Goldman MHS, De Vries RP, Goldman GH. The influence of Aspergillus niger transcription factors AraR and $\mathrm{X} \ln \mathrm{R}$ in the gene expression during growth in d-xylose, 1-arabinose and steam-exploded sugarcane bagasse. Fungal Genet Biol. 2013;60:29-45.

33. Pullan ST, Daly P, Delmas S, Ibbett R, Kokolski M, Neiteler A, van Munster JM, Wilson R, Blythe MJ, Gaddipati S, Tucker GA, Archer DB. RNAsequencing reveals the complexities of the transcriptional response to lignocellulosic biofuel substrates in Aspergillus niger. Fungal Biol Biotechnol. 2014;1:3.

34. Bischof R, Fourtis L, Limbeck A, Gamauf C, Seiboth B, Kubicek CP. Comparative analysis of the Trichoderma reesei transcriptome during growth on the cellulase inducing substrates wheat straw and lactose. Biotechnol Biofuels. 2013;6:127.

35. Ries L, Pullan ST, Delmas S, Malla S, Blythe MJ, Archer DB. Genomewide transcriptional response of Trichoderma reesei to lignocellulose using 
RNA sequencing and comparison with Aspergillus niger. BMC Genom. 2013; 14:541.

36. Ramos ASP, Chambergo FS, Bonaccorsi ED, Ferreira AJS, Cella N, Gombert AK, Tonso A, El-Dorry H. Oxygen- and glucose-dependent expression of Trhxt1, a putative glucose transporter gene of Trichoderma reesei. Biochemistry. 2006;45:8184-92.

37. Zhang W, Kou Y, Xu J, Cao Y, Zhao G, Shao J, Wang H, Wang Z, Bao X, Chen G, Liu W. Two major facilitator superfamily sugar transporters from Trichoderma reesei and their roles in induction of cellulase biosynthesis. J Biol Chem. 2013;288:32861-72.

38. Vankuyk PA, Diderich JA, MacCabe AP, Hererro O, Ruijter GJG, Visser J. Aspergillus niger $m s t A$ encodes a high-affinity sugar $/ \mathrm{H}+$ symporter which is regulated in response to extracellular pH. Biochem J. 2004;379(Pt 2):375-83. 39. Hector RE, Mertens JA, Bowman MJ, Nichols NN, Cotta MA, Hughes SR. Saccharomyces cerevisiae engineered for xylose metabolism requires gluconeogenesis and the oxidative branch of the pentose phosphate pathway for aerobic xylose assimilation. Yeast. 2011;28:645-60.

40. Maier A, Völker B, Boles E, Fuhrmann GF. Characterization of glucose transport in Saccharomyces cerevisiae with plasma membrane vesicles (countertransport) and intact cells (initial uptake) with single Hxt1, Hxt2, Hxt3, Hxt4, Hxt6, Hxt7 or Gal2 transporters. FEMS Yeast Res. 2002;2:539-50. 41. van Peij N. The transcriptional activator XlnR regulates both xylanolytic and endoglucanase gene expression in Aspergillus niger. Appl Environ Microbiol. 1998;64:3615-9.

42. Derntl C, Gudynaite-Savitch L, Calixte S, White T, Mach RL, Mach-Aigner AR. Mutation of the xylanase regulator 1 causes a glucose blind hydrolase expressing phenotype in industrially used Trichoderma strains. Biotechnol Biofuels. 2013;6:62.

43. Stricker AR, Grosstessner-Hain K, Würleitner E, Mach RL. Xyr1 (xylanase regulator 1) regulates both the hydrolytic enzyme system and d-xylose metabolism in Hypocrea jecorina. Eukaryot Cell. 2006;5:2128-37. 44. Wang C, Bao X, Li Y, Jiao C, Hou J, Zhang Q, Zhang W, Liu W, Shen Y. Cloning and characterization of heterologous transporters in Saccharomyces cerevisiae and identification of important amino acids for xylose utilization. Metab Eng. 2015;30:79-88.

45. Knoshaug EP, Vidgren V, Magalhães F, Jarvis EE, Franden MA, Zhang M, Singh A. Novel transporters from Kluyveromyces marxianus and Pichia guilliermondii expressed in Saccharomyces cerevisiae enable growth on 1arabinose and d-xylose. Yeast. 2015;32:615-28. 
46. Kasahara T, Shimogawara K, Kasahara M. Crucial effects of amino acid side chain length in transmembrane segment 5 on substrate affinity in yeast glucose transporter Hxt7. Biochemistry. 2011;50:8674-81.

47. UniProt: a hub for protein information. Nucleic Acids Res. 2014;43(Database issue):D204-12.

48. Simossis VA, Heringa J. PRALINE. a multiple sequence alignment toolbox that integrates homology-extended and secondary structure information. Nucleic Acids Res. 2005;33(Web Server issue):W289-94. 49. Finn RD, Clements J, Eddy SR. HMMER web server: interactive sequence similarity searching. Nucleic Acids Res. 2011;39(Web Server issue):W29-37.

50. Andersen MR, Salazar MP, Schaap PJ, De Van, Vondervoort PJI, Culley D, Thykaer J, Frisvad JC, Nielsen KF, Albang R, Albermann K, Berka RM, Braus GH, Braus-Stromeyer SA, Corrochano LM, Dai Z, Van Dijck PWM, Hofmann G, Lasure LL, Magnuson JK, Menke H, Meijer M, Meijer SL, Nielsen JB, Nielsen ML, Van Ooyen AJJ, Pel HJ, Poulsen L, Samson RA, Stam H, Tsang A, et al. Comparative genomics of citric-acid-producing Aspergillus niger ATCC 1015 versus enzyme-producing CBS 513.88. Genome Res. 2011;21:885-97.

51. Koike H, Aerts A, LaButti K, Grigoriev IV, Baker SE. Comparative genomics analysis of Trichoderma reesei strains. Ind Biotechnol. 2013;9:35267.

52. Nordberg H, Cantor M, Dusheyko S, Hua S, Poliakov A, Shabalov I, Smirnova T, Grigoriev IV, Dubchak I. The genome portal of the Department of Energy Joint Genome Institute: 2014 updates. Nucleic Acids Res. 2014;42(Database issue):D26-31.

53. Hasper AA, Trindade LM, van der Veen D, van Ooyen AJJ, de Graaff LH. Functional analysis of the transcriptional activator XlnR from Aspergillus niger. Microbiology. 2004;150(Pt 5):1367-75.

54. Steiger MG, Vitikainen M, Uskonen P, Brunner K, Adam G, Pakula T, Penttilä M, Saloheimo M, Mach RL, Mach-Aigner AR. Transformation system for Hypocrea jecorina (Trichoderma reesei) that favors homologous integration and employs reusable bidirectionally selectable markers. Appl Environ Microbiol. 2011;77:114-21.

55. Mello-de-Sousa TM, Rassinger A, Pucher ME, dos Santos Castro L, Persinoti GF, Silva-Rocha R, Poças-Fonseca MJ, Mach RL, Nascimento Silva $\mathrm{R}$, Mach-Aigner AR. The impact of chromatin remodelling on cellulase expression in Trichoderma reesei. BMC Genom. 2015;16:588. 56. Pontecorvo G, Roper JA, Chemmons LM, Macdonald KD, Bufton AWJ. The genetics of Aspergillus nidulans. Adv Genet. 1953;5:141-238. 
57. Vishniac W, Santer M. The thiobacilli. Bacteriol Rev. 1957;21:195-213.

58. Mandels M. Applications of cellulases. Biochem Soc Trans. 1985;13:414-6.

59. Hamacher T, Boles E, Gárdonyi M, Hahn-Hägerdal B, Becker J.

Characterization of the xylose-transporting properties of yeast hexose

transporters and their influence on xylose utilization. Microbiology.

2002;148:2783-8.

60. Schuster A, Bruno KS, Collett JR, Baker SE, Seiboth B, Kubicek CP,

Schmoll M. A versatile toolkit for high throughput functional genomics with

Trichoderma reesei. Biotechnol Biofuels. 2012;5:1.

61. Gietz RD, Woods RA. Transformation of yeast by lithium

acetate/singlestranded carrier DNA/polyethylene glycol method. Methods

Enzymol. 2002;350:87-96.

62. Boeke JD, Trueheart J, Natsoulis G, Fink GR. 5-Fluoroorotic acid as a selective agent in yeast molecular genetics. Methods Enzymol. 1987;154:16475.

63. Walsh MC, Smits HP, Scholte M, van Dam K. Affinity of glucose transport in Saccharomyces cerevisiae is modulated during growth on glucose. $\mathrm{J}$

Bacteriol. 1994;176:953-8.

64. Mach-Aigner AR, Omony J, Jovanovic B, van Boxtel AJB, de Graaff LH. $\mathrm{d}$-Xylose concentration-dependent hydrolase expression profiles and the function of CreA and XlnR in Aspergillus niger. Appl Environ Microbiol. 2012;78:3145-55.

65. Steiger MG, Mach RL, Mach-Aigner AR. An accurate normalization strategy for RT-qPCR in Hypocrea jecorina (Trichoderma reesei). $\mathrm{J}$

Biotechnol. 2010;145:30-7.

66. Martinez D, Berka RM, Henrissat B, Saloheimo M, Arvas M, Baker SE, Chapman J, Chertkov O, Coutinho PM, Cullen D, Danchin EGJ, Grigoriev IV, Harris P, Jackson M, Kubicek CP, Han CS, Ho I, Larrondo LF, de Leon AL, Magnuson JK, Merino S, Misra M, Nelson B, Putnam N, Robbertse B, Salamov AA, Schmoll M, Terry A, Thayer N, Westerholm-Parvinen A, et al. Genome sequencing and analysis of the biomass-degrading fungus Trichoderma reesei (syn. Hypocrea jecorina). Nat Biotechnol. 2008;26:553-60.

67. van Peij NN, Visser J, de Graaff LH. Isolation and analysis of $x \ln R$, encoding a transcriptional activator co-ordinating xylanolytic expression in Aspergillus niger. Mol Microbiol. 1998;27:131-42.

68. Sievers F, Wilm A, Dineen D, Gibson TJ, Karplus K, Li W, Lopez R, McWilliam H, Remmert M, Soding J, Thompson JD, Higgins DG. Fast, scalable generation of high-quality protein multiple sequence alignments using clustal omega. Mol Syst Biol. 2011;7:539. 


\section{Chapter 5}

Overexpression of the Aspergillus niger GatA transporter leads to preferential use of D-galacturonic acid over Dxylose

Jasper Sloothaak, Mike Schilders, Peter J. Schaap and Leo H. de Graaff

Sloothaak et al. AMB Express (2014) Volume 4 


\title{
Overexpression of the Aspergillus niger GatA transporter leads to preferential use of D- galacturonic acid over D-xylose
}

\begin{abstract}
Pectin is a structural heteropolysaccharide of the primary cell walls of plants and as such is a significant fraction of agricultural waste residues that is currently insufficiently used. Its main component, D-galacturonic acid, is an attractive substrate for bioconversion. The complete metabolic pathway is present in the genome of Aspergillus niger, that is used in this study. The objective was to identify the D-galacturonic acid transporter in $A$. niger and to use this transporter to study D-galacturonic acid metabolism.

We have functionally characterized the gene An14g04280 that encodes the Dgalacturonic acid transporter in $A$. niger. In a mixed sugar fermentation it was found that the An14g04280 overexpression strain, in contrast to the parent control strain, has a preference for D-galacturonic acid over D-xylose as substrate. Overexpression of this transporter in A. niger resulted in a strong increase of D-galacturonic acid uptake and induction of the D-galacturonic acid reductase activity, suggesting a metabolite controlled regulation of the endogenous D-galacturonic acid catabolic pathway.
\end{abstract}




\section{Background}

Global limits in food and energy availability are becoming a major concern. Arable land is needed for production of food and large waste streams come from processing of our largest food resources, like grain, maize, potato and rice. These waste streams are currently insufficiently exploited and mainly used for feed and energy production [1]. Alternatively, agricultural waste streams can be used as substrate for fermentative production of chemicals by microorganisms. Historically, Aspergillus species are used for the production of food additives and platform chemicals such as citric acid (A. niger) and itaconic acid (A.terreus) [2]. The filamentous fungus Aspergillus niger is additionally exploited for production of enzymes for food and feed applications, many of which have been rewarded the GRAS (Generally Recognized As Safe) status [3]. Grain, maize and rice waste stream material contains around $40 \%$ cellulose, $35 \%$ hemicellulose, $20 \%$ lignin and 5\% pectin, while waste streams from other plants, such as sugar beet and potato, contain around $20 \%$ cellulose and hemicellulose, less than $1 \%$ lignin and $30 \%$ to $40 \%$ pectin $[1,4,5]$. A. niger has a high capacity for degrading the hemicellulose, pectin and, to a lesser extend, cellulose fractions as the genome of $A$. niger encodes multiple gene variants of the enzymes required for the efficient degradation of these polysaccharides [6]. D-glucose and D-xylose, released from plant wall material, are carbon sources that will yield high energy upon being metabolized. The genome of $A$. niger is wired to specifically produce and secrete the enzymes involved in degradation of the cellulose and hemicellulose fractions to release D-glucose and D-xylose. These sugars are then taken up and metabolized, before energy is invested in the release of other carbon sources from polysaccharides. This preferential uptake is regulated by the interplay of activating and repressing transcription factors that respond to extracellular concentrations of inducers and sugars. Expression of genes that code for enzymes involved in cellulose and hemicellulose degradation in A. niger is regulated by the transcription factor $X \ln R$ [7]. $X \ln R$ is activated by inducers derived from xylan, like $\mathrm{D}$-xylose, though at a higher concentration this activation is attenuated by CreA [8-10]. Tight regulation of the specific genes encoding proteins involved in the release, uptake and metabolism of sugars gives the fungus the ability to utilize the available sugars in a physiologically most efficient way. However, circumventing this regulatory mechanism would potentially lead to simultaneous uptake of all sugar substrates and improved fermentation yields. Genes encoding proteins involved in the degradation of pectin and the D-galacturonic acid metabolic pathway are found to be highly upregulated when $A$. niger is growing in the presence of D-galacturonic acid, in comparison to when the fungus is growing in the presence of D-glucose or D- 
fructose (Table 1). Three genes among the genes that are upregulated are coding for proteins with a strong similarity to transporters $[11,12]$. In this study we have functionally identified the D-galacturonic mono-acid sugar transporter by overexpression in A. niger. The effect of the increased uptake of D-galacturonic acid on the preferential uptake and metabolism of substrates has been assessed by mixed sugar fermentations and enzyme activity measurements.

Table 1 Data used for selection of putative D-galacturonic acid transporters (Martens-Uzunova and Schaap 2008)

\begin{tabular}{lllll}
\hline \multirow{2}{*}{ Locus tag } & Functional annotation & \multicolumn{3}{l}{ Relative expression $\mathbf{t}=\mathbf{4} \mathbf{~}$ after induction } \\
\cline { 3 - 5 } & galA & poly-galA & pectin \\
\hline An07g00780 & $\begin{array}{l}\text { Major facilitator superfamily strong similarity } \\
\text { to monocarboxylate transporter }\end{array}$ & 23.10 & 9.98 & 5.27 \\
An14g04280 & $\begin{array}{l}\text { Major facilitator superfamily strong similarity } \\
\text { to hexose transporter }\end{array}$ & 26.53 & 22.74 & 27.95 \\
An03g01620 & $\begin{array}{l}\text { Major facilitator superfamily strong similarity } \\
\text { to hexose transporter }\end{array}$ & 6.50 & 1.345 & 37.54 \\
\hline
\end{tabular}




\section{Results}

\section{Strains that overexpress GatA have increased D-galacturonic acid uptake in shake flask cultures}

To study the effect of the expression of the putative D-galacturonic acid transporter-encoding gene, the D-galacturonic acid concentration in the medium was measured during growth. A faster uptake of D-galacturonic acid from the medium was observed for the cultures with the An14g04280 overexpression strains (transformant) in comparison to the NW185 PYR A+control strain. At 6 hours after induction, the strains that overexpress

An14g04280 had taken up over $50 \%$ of Dgalacturonic acid present at the start of the cultivation, while no D-galacturonic acid had been taken up by the control strain. The strains containing the other constructs did not show any difference in D-galacturonic acid uptake in comparison to the NW185 PYR A+ control strain. The effect of the An14g04280

overexpression strain is most pronounced between time points 6

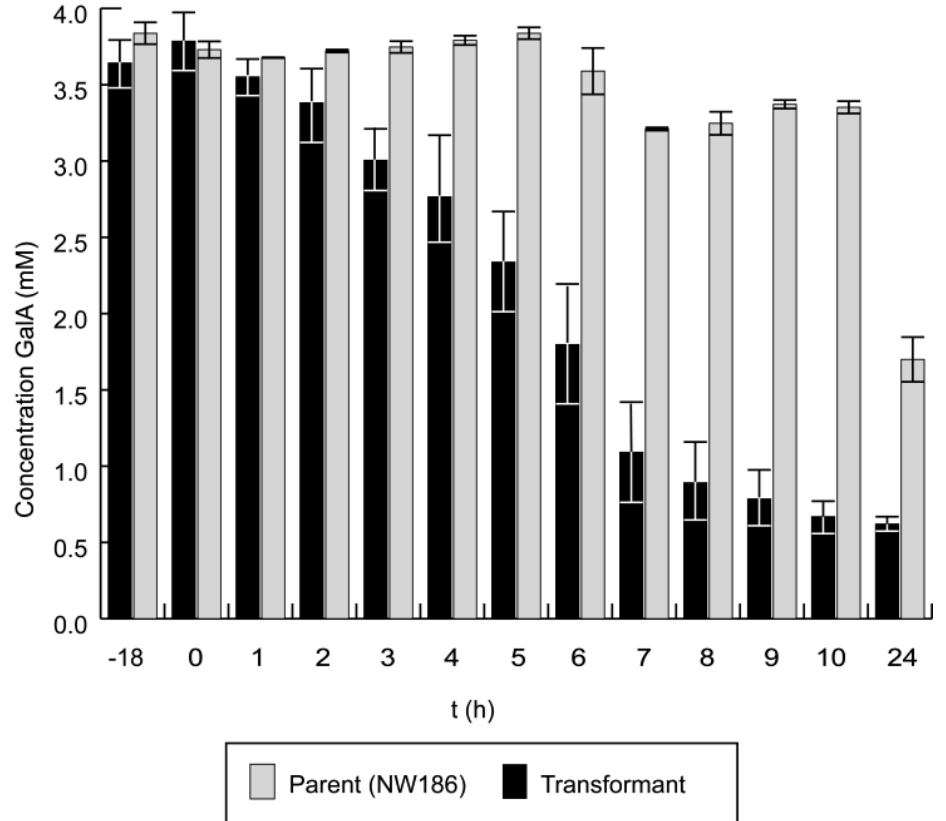

Figure 2 Concentration of GalA in the medium during growth on sorbitol and GalA. Induction of expression system with $\mathrm{D}$-xylose at $\mathrm{T}=0$. Overexpression strains of gatA (number of replicates $=4$ ) in comparison to the control NW185 PYR A+ (number of replicates =2).

and $30 \mathrm{~h}$. A second experiment was performed to get a more time resolved determination of the uptake of D-galacturonic acid by the best performing transformant strain (JS013). The difference in D-galacturonic acid uptake starts to become evident from 4 hours after induction, with the highest contrast between 5 and 6 hours after induction (Figure 2). To study the effect of An14g04280 in the uptake of D-galacturonic acid during growth on more complex mixed sugar substrates, JS013 was grown in the presence of a substrate mix (SCH), mimicking 

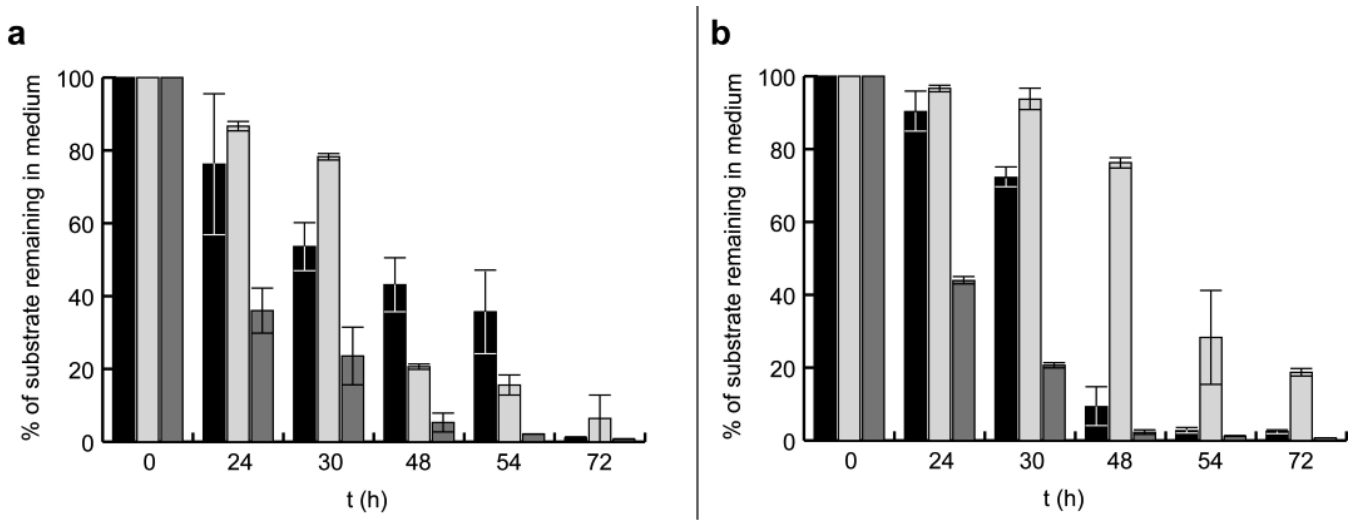

Xylose $\square$ Galacturonic acid $\square$ Glucose

Figure 3 Percentage remaining in medium of the three most important substrates from the synthetic cell-wall hydrolysate for gatA overexpression strain (a) (number of replicates $=2$ ) and the parent (NW185 PYR A+ strain) (b) (number of replicates $=2$ ).

the composition of a plant cell wall hydrolysate [13]. When comparing the uptake of the three main components D-glucose, D-xylose and D-galacturonic acid between the transformants and the control strain, a clear difference is found (Figure 3a and b). Till 24 hours after inoculation, substrate uptake is similar for all strains. After 30 hours, differences in D-galacturonic acid and D-xylose uptake become evident. At time point 48 hours, the control strain has almost depleted the D-xylose and most of the D-galacturonic acid remains in the medium, while the transformant has consumed $80 \%$ of the D-galacturonic acid and most D-xylose remains in the medium. While D-glucose and D-xylose are depleted in the control culture after 54 hours, depletion of D-xylose in the transformant cultures can only be seen at time point $72 \mathrm{~h}$. The biomass formed and the citrate produced were measured for the An14g04280 overexpression strain and the control strain. Recovery of substrate in biomass and citrate produced was $35 \%$ for the control strain and 30\% for the transformants. It was found that for the overexpression strain $30 \%$ of this is citrate and $70 \%$ is biomass, while for the control strain, $10 \%$ is citrate and $90 \%$ is biomass (Figure 4). Cmoles that are not accounted for are assumed to be $\mathrm{CO}_{2}$ and enzyme production (not measured). For these calculations the generalized molecular formula for fungal biomass $\mathrm{CH}_{1.72} \mathrm{O}_{0.55} \mathrm{~N}_{0.17}$ was used [14]. 

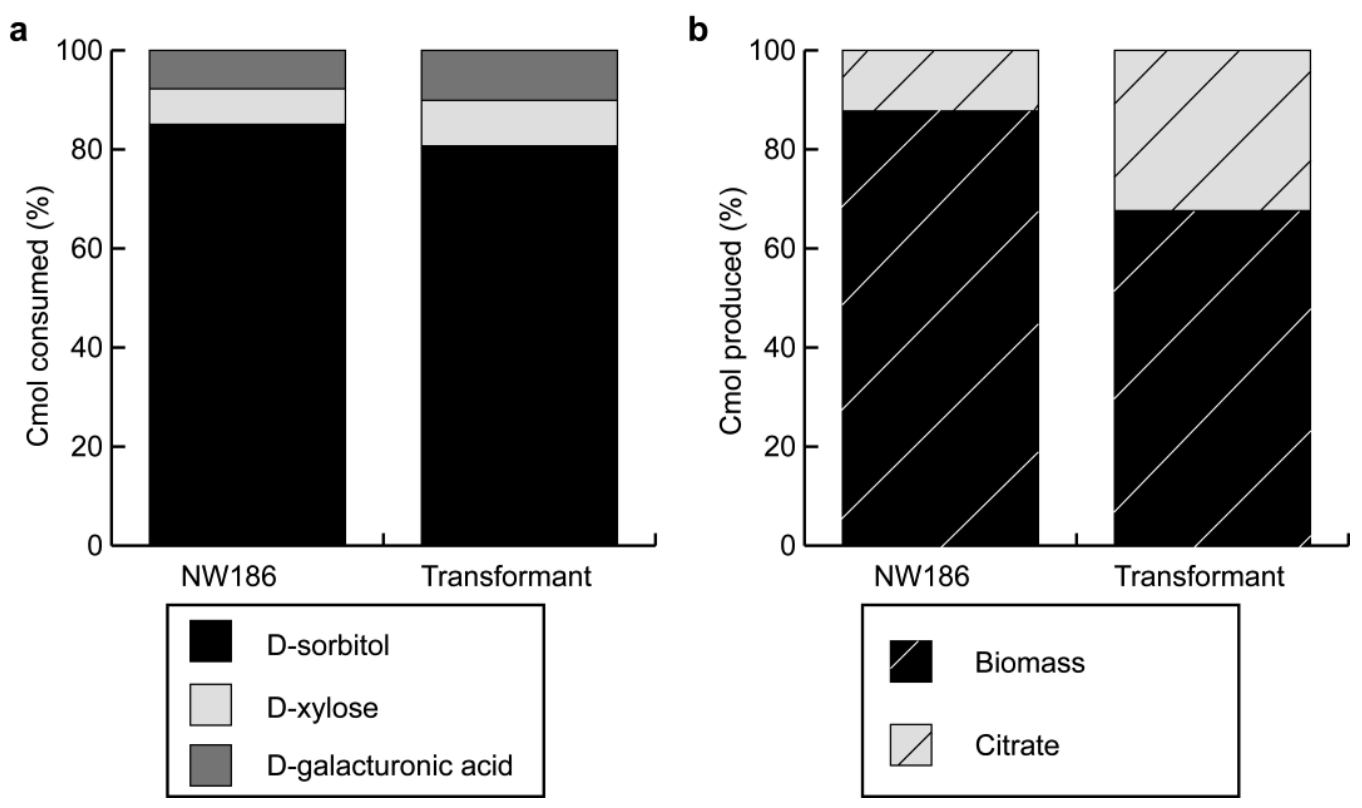

Figure 4 Carbon consumed (a) and produced (b), expressed in Cmoles, for control strain (NW185 PYR A+ strain) and transformant JS013 after $72 \mathrm{~h}$ of growth on $\mathrm{SCH}$ medium. Samples taken from direct cultures, D-xylose in the mixed substrate medium serves as an inducer of the $x \ln D$ promoter. Totals represent 0.1 Cmoles

\section{Growth of transporter overexpression strains in fermentors}

When strains were grown in the presence of equimolar concentration of D-xylose and D-galacturonic acid in fermentors, preferential uptake of D-galacturonic acid over D-xylose of JS013 in comparison to NW185 PYR A+control strain is observed. This is deduced from measured concentrations of D-galacturonic acid and $\mathrm{D}$-xylose, but also from an increase of the $\mathrm{pH}$ of the medium, after $\mathrm{D}$ galacturonic acid is taken up (Figure 5). Increased activity of the D-galacturonic acid metabolic pathway is observed for JS013 transformant strain in comparison to the control. For the first 4 hours after transfer to the fermentors, no difference in D-galacturonic acid reductase activity could be seen. 8 hours after transfer, however, specific activity of JS013 is two-fold higher than that of the control strain. The concentration of $\mathrm{CO}_{2}$ in the off gas went up to 0.35 percent by volume in the first two hours and slowly down to 0.2 percent by volume in the next 6 hours, while ambient concentration was 0.04 percent by volume (Figure 6). 

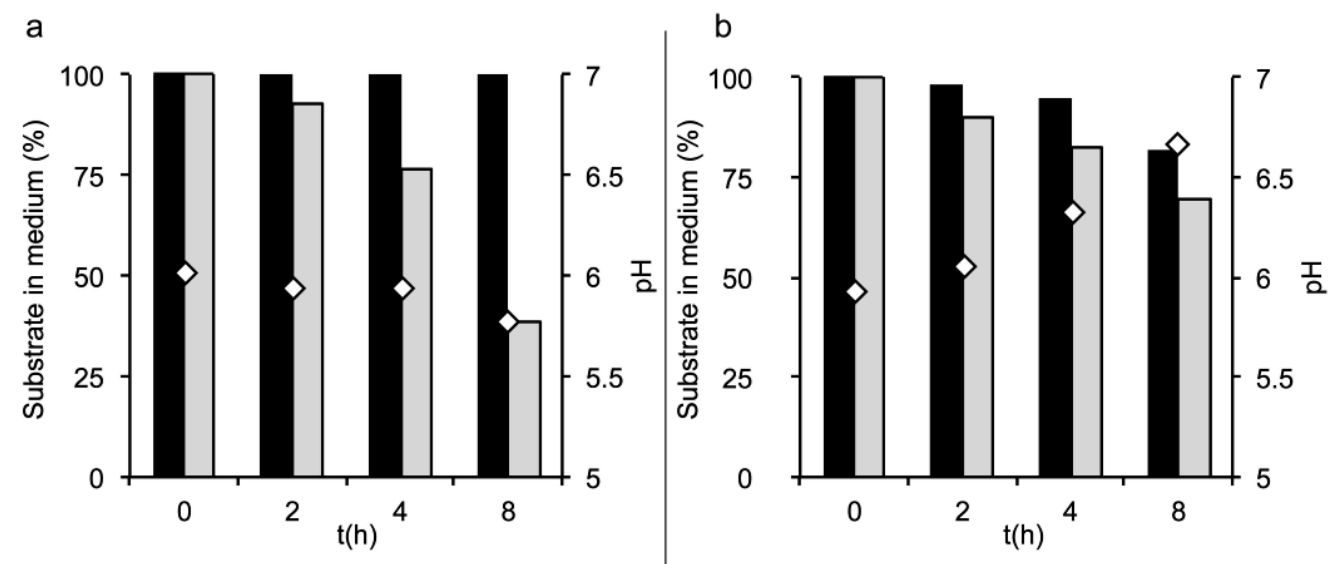

- Galacturonic acid $\quad$ axylose $\diamond \mathrm{pH}$

Figure 5 Substrate remaining in culture medium and $\mathrm{pH}$ during growth in fermentors of the control, (NW185 PYR A+ strain) (a) and overexpression strain, JS013 (b). The strains were pre-grown and mycelium was transferred at T $=0$ to fermentors containing D-xylose and D-galacturonic acid as substrates
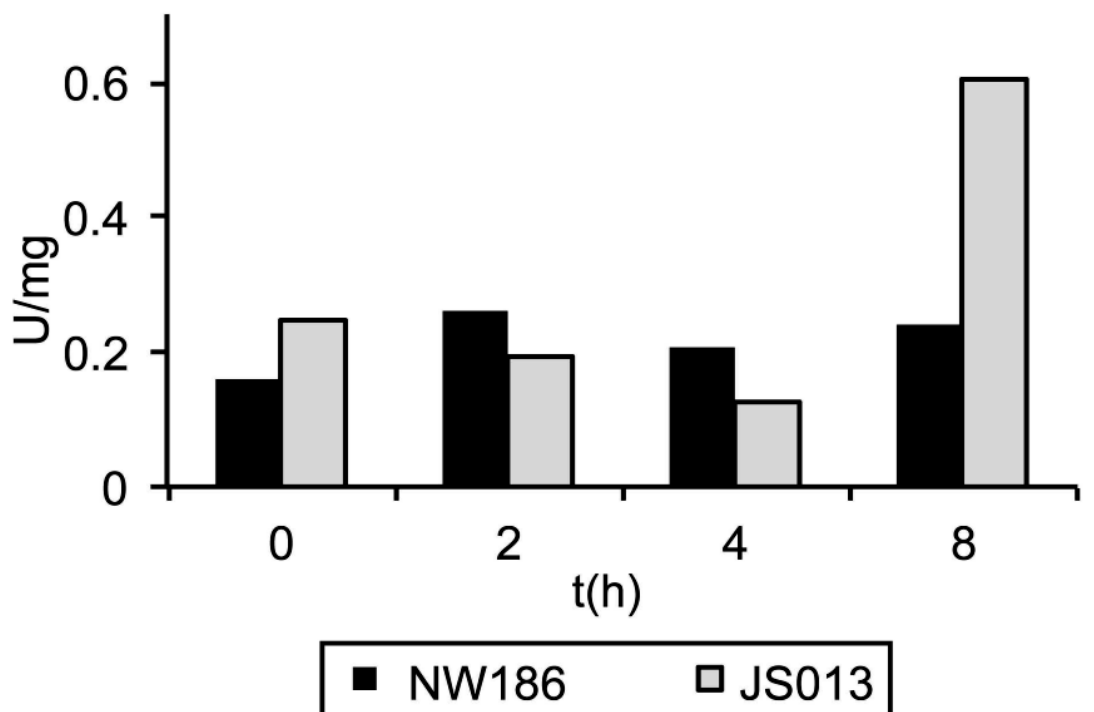

Figure 6 Galacturonic acid reductase activity (Bars; left $\mathrm{Y}$ axis) and $\mathrm{CO} 2$ in offgas (Diamonds; right $\mathrm{Y}$ axis). Samples were taken during growth on $25 \mathrm{mM}$ of D-xylose and $25 \mathrm{mM}$ of $\mathrm{D}$-galacturonic acid as a carbon source in fermentors 


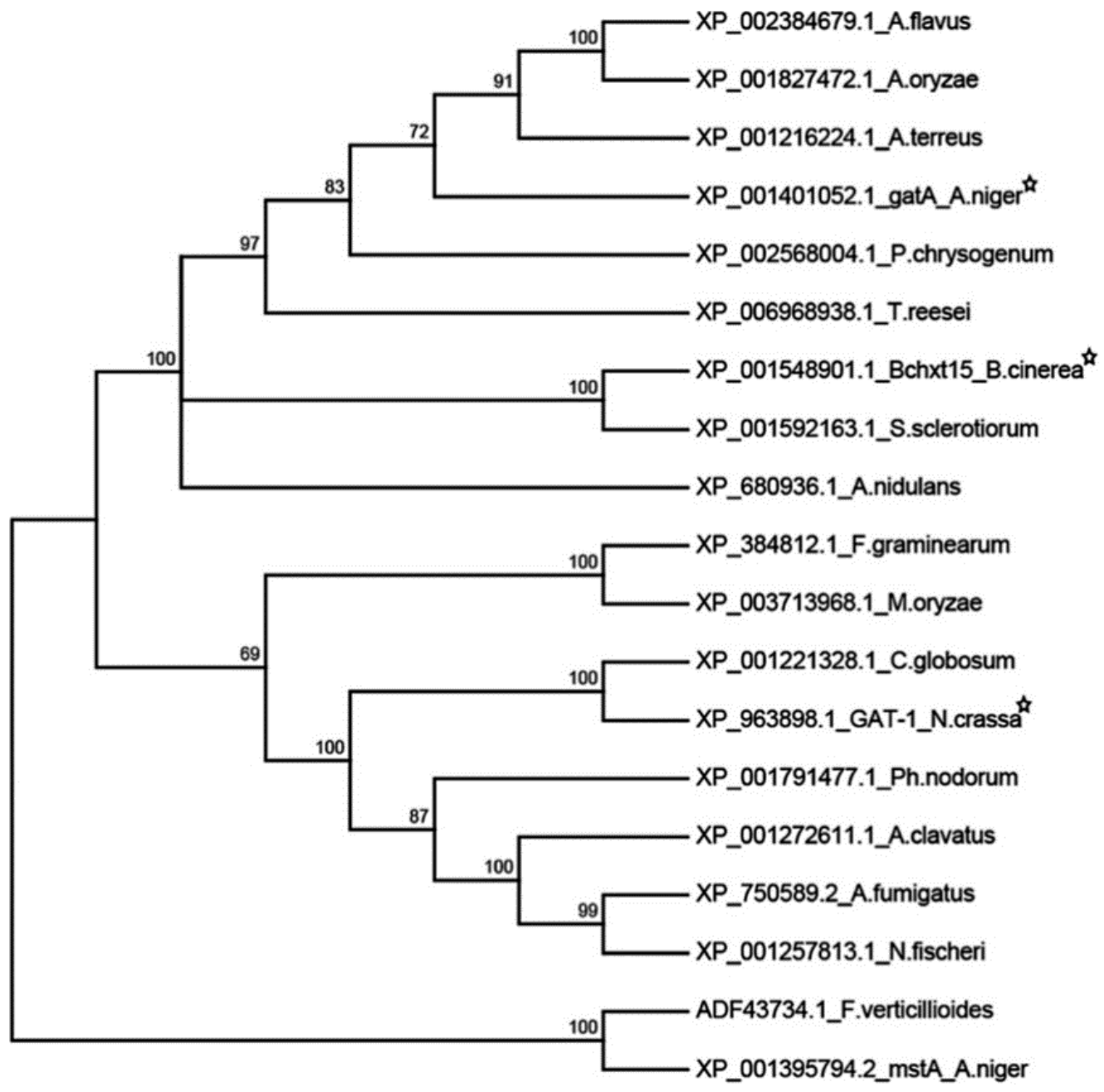

Figure 7 Neighbor-joining tree of GatA and 17 putative orthologs, constructed with 1000 bootstrap replicates using MEGA6.06 (Tamura et al. 2013), and a characterized high-affinity glucose transporter protein ( $m s t A \_$A.niger) as an outgroup (vanKuyk et al., 2004). Stars indicate the three currently characterized D-galacturonic acid transporters. Bootstrap values are indicated at the branchpoints. Labels indicate Protein Accession numbers followed by species abbreviations. Abbreviations: Aspergillus clavatus; A. clavatus, Aspergillus flavus; A. flavus, Aspergillus fumigatus; A. fumigatus, Aspergillus nidulans; A. nidulans, Aspergillus niger; A. niger, Aspergillus oryzae; A. oryzae, Aspergillus terreus; $A$. terreus, Botrytis cinerea; B. cinerea, Chaetomium globosum; C. globosum, Fusarium verticilliodes; $F$. verticilliodes, Neosartorya fischeri; $N$. fischeri, Neurospora crassa; N. crassa, Penicillium chrysogenum; P. chrysogenum, Phaeosphaeria nodorum; Ph. nodorum, Sclerotinia sclerotiorum; S. sclerotiorum, Trichoderma reesei; T. reesei 


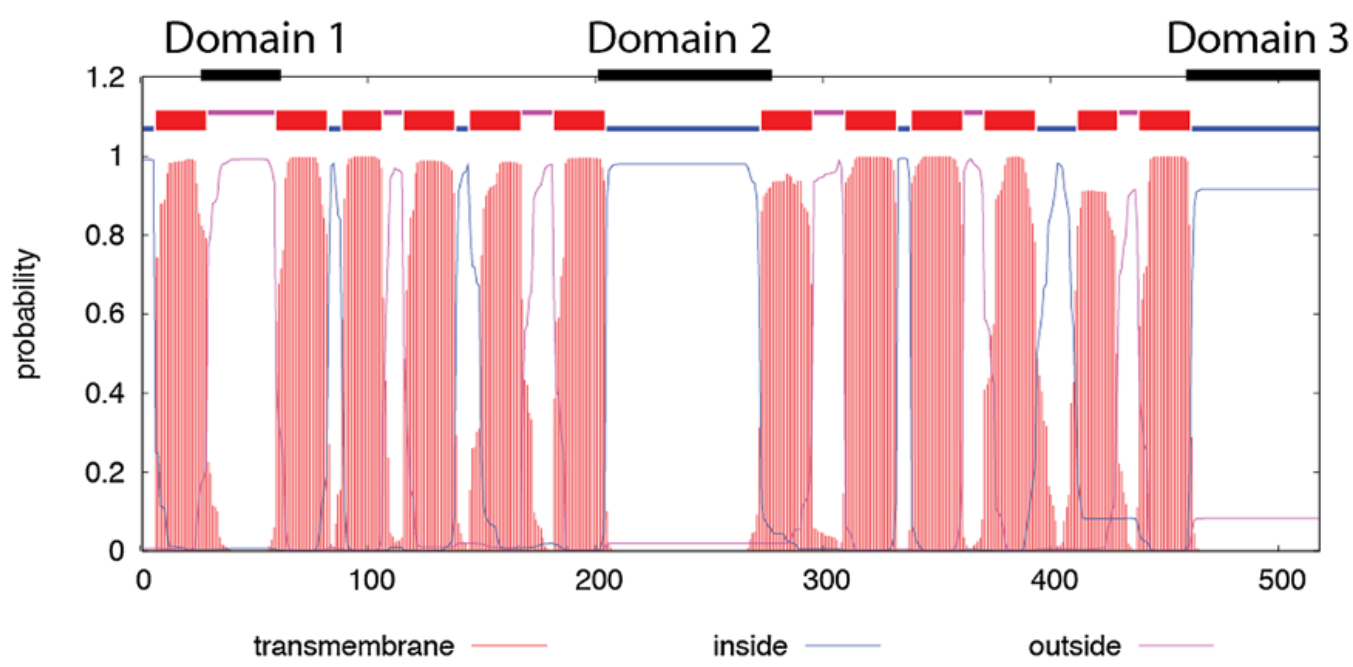

Figure 8 Transmembrane domain prediction and indication of the two interacting domains $(1,3)$ and the putative substrate binding domain (2) used for comparison of the putative GatA orthologs (Viklund and Elofsson 2004)

\section{Phylogenetic analysis of GatA}

In Botrytis cinerea and Neurospora crassa, D-galacturonic acid transporter proteins have been functionally identified $[15,16]$. After import, D-galacturonic acid is catabolized by three key enzymes: D-galacturonic acid reductase, Lgalactonate dehydratase and 2-keto-3-deoxy-galactonate aldolase, in A. niger encoded by gaaA, gaaB and gaaC respectively. Orthologs of the gaaA and gaaC genes have been identified in $B$. cinerea and $N$. crasssa and 16 other fungal species that all have a conserved bidirectional organization of their gaaA-gaaC promoter region [11]. For each of these 18 species a bidirectional best BLAST hits procedure [17] yielded a putative GatA ortholog. Orthologous amino acid sequences were aligned with Clustal [18] and a phylogenetic tree was constructed (Figure 7). All orthologs have 12 predicted transmembrane helices [19] separating two interacting domains and the substrate-binding domain [20] (Figure 8). In these three domains the three characterized D-galacturonic acid transporters share 18 conserved residues not present in the A. niger MstA Dglucose transporter protein sequence, which was used as an out-group. The presence of this motif was used to assess the other 16 putative orthologs. Fifteen putative orthologs shared 8 of these residues, excluding the Fusarium verticilliodes putative ortholog which clusters with the D-glucose transporter MstA (Figure 7) and can be a transporter for another sugar. 


\section{Discussion}

Three genes that potentially encode the $\mathrm{D}$-galacturonic acid transporter in $A$. niger are investigated. A. niger transformants that overexpress the An14g04280 gene under control of the $\mathrm{D}$-xylose induced $x \ln D$ promoter show a significant increase in D-galacturonic acid uptake. From this we conclude that this gene, gatA, encodes for the D-galacturonic acid transporter, GatA. By analysis of the amino acid sequence using the $\alpha$-helical transmembrane protein topology prediction software (PRODIV-TNHMM) [19] GatA is predicted to be part of the Major Facilitator Superfamily (MFS). MFS transporter proteins consist of 12 transmembrane helices and have both $\mathrm{C}$ - and $\mathrm{N}$-termini located in the cytoplasm. This study shows that the overexpression of gatA, using a D-xylose inducible promoter, leads to differences in the uptake of sugars when the strains are grown on various mixed sugar substrates. GatA overproducing strains preferentially use D-galacturonic acid over D-xylose, while the wild type strains prefer D-xylose. D-xylose is used for overexpression of the GatA transporter in all growth experiments and it is also used as a substrate. However, the main carbon source present in the medium is sorbitol, a non-inducing, non-repressing carbon source. Our extensive previous studies have shown that in this condition induction of expression occurs at concentrations of 0.1 to $50 \mathrm{mM}$ D-xylose [12,21]. The increased D-galacturonic acid uptake of the transformant was not reflected in the basal GaaA enzyme activity during the first hours of growth on D-xylose and Dgalacturonic acid. It is, however, reflected in an increased GaaA enzyme activity after 8 hours. This time delay suggests that GaaA enzyme activity is not limiting D-galacturonic acid uptake and metabolism during the first few hours of growth on these substrates and that regulation of expression of GaaA is taking place "at the gate", via import of D-galacturonic acid. The repression of induction of GaaA in the presence of D-xylose in the parent strain, is bypassed by an increased influx of substrate in the GatA overexpressing strain, suggesting that the endogenous D-galacturonic acid catabolic pathway is controlled by a pathway intermediate such as L-galactonate or keto-deoxy-L-galactonate as has been suggested for $T$. reesei [22]. Several repressing and inducing regulatory systems are known to be functioning in Aspergillus niger. The glucose carbon catabolite repressor creA is the most studied and is known to be dominant in most cases [8]. The promoter region of the gatA gene shows two consensus sequences for binding of this protein, which suggests that D-glucose is repressing the expression of the endogenous gene. The $x \ln R$ xylanolytic induction system is also well studied and an inducer consensus binding sequence is known [10]. This consensus sequence cannot be found in the upstream region of the endogenous gatA gene and it is therefore not induced by the presence of D-xylose. Additionally, GatA 
expression has been found to be strictly co-regulated with D-galacturonic acid catabolic enzymes and a number of extracellular galacturonases [11]. The regulator of the D-galacturonic acid metabolism, however, remains unknown for now. We have investigated the application of our findings by growing our strains on a synthetic medium mimicking a plant cell wall hydrolysate. Under these conditions the gatA overexpressing strains preferentially use D-galacturonic acid over D-xylose. In these experiments it was found that GatA overexpression strains had a higher citric acid yield. While the effect seems pronounced on a simulated hydrolysate substrate, further experiments are needed to study the benefit on second-generation feedstocks with high $\mathrm{D}$-galacturonic acid content like sugar beet pulp to investigate the potential of GatA in biotechnological applications. 


\section{Methods}

\section{Strains}

All Aspergillus strains used are descendants of A. niger N400 (CBS 120.49). A niger strain NW185 cspA1, fwnAl, goxC17, prtF28) was derived from NW131 and has been described by Ruijter et al. 1999 [9]. The recipient strain in all transformation experiments NW186 ( $\triangle \operatorname{argB}$; pyrA6; prtF28; goxC17; $\operatorname{csp} A 1)$ is an $\arg B$ and $p y r A$ derivative of NW185.

\section{Vector construction}

Primers used in the construction of vectors are summarized in Table 2. Plasmids used for transformations of $A$. niger were constructed according to the general scheme given in Figure 1. Martens-Uzunova and Schaap was the source for the selection of the putative transporters [11]; An14g04280 and An03g01620 were

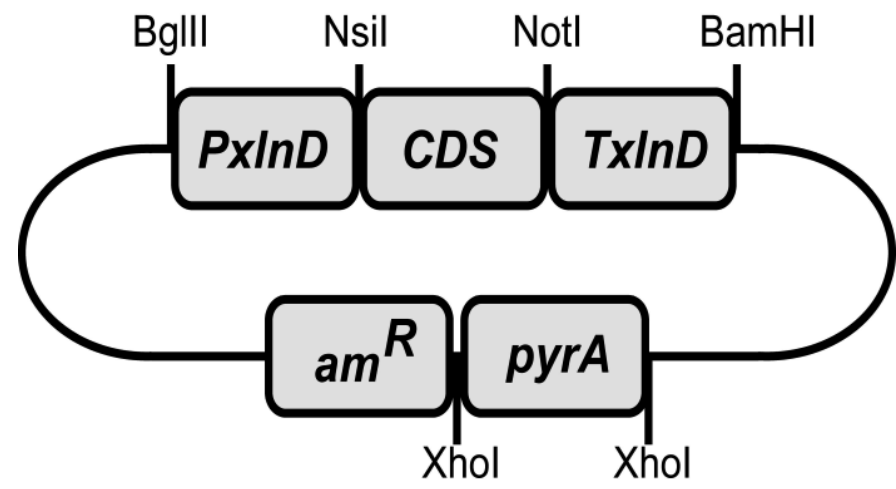
obtained from the Aspergillus genome

Figure 1 Schematic representation of Funbrick expression vector. Restriction sites flank functional modules. Capital P and T represent promoter and terminator. Bacterial marker: amR. Fungal uridine auxotrophy complementation marker: pyrA database [23,24]. These sequences were used to synthesize the respective coding sequences that were modified to remove conflicting restriction sites (BaseClear, Leiden, Netherlands). The An07g00780 fragment was amplified by PCR using primers JS_gatA_FW and JS_gatA_RV and $A$. niger NW186 genomic DNA as template and was subcloned into a pJET1.2 vector (Thermo scientific). The BglII restriction site was removed from the coding sequence by using the Quikchange Lightning Site-directed mutagenesis kit (Agilent Technologies) with the primers JS_gatCmut2_FW and JS_gatCmut2_RV. The PCR fragments were cloned into a pUC19 derived expression vector under control of a modified $x \ln D$ promoter and the $x \ln D$ terminator of $A$. niger. Plasmids were propagated in DH5a E. coli. The medium used for $E$. coli growth was LB $(10 \mathrm{~g} / \mathrm{L}$ Bacto tryptone, $5 \mathrm{~g} / \mathrm{L}$ Yeast extract, $10 \mathrm{~g} / \mathrm{L} \mathrm{NaCl}$ ) supplemented with $100 \mathrm{mg} / \mathrm{L}$ ampicillin when appropriate. 


\section{Transformation of Aspergillus niger}

For the transformation of $A$. niger NW186, protoplasts were generated using Novozyme-234 lysing enzyme cocktail. The An14g04280, An03g01620 and An07g00780 constructs were introduced in A. niger by transformation [25] using the pyrA gene as a primary selection marker, relieving uridine auxotrophy. To generate the parent control reference strain, NW186 was transformed using pGW635, the resulting NW185 PYR A+strain was used as a control strain in our studies. Colonies were randomly picked from the primary transformation plates and re-plated on selective medium to purify the single colonies.

Table 2 Primers used in this study

\begin{tabular}{ll}
\hline Primer & Sequence \\
\hline JS_gatC_FW & 5'-GAGAATGCATATGTCTGAGCCTAAGAACCAGC-3' \\
JS_gatC_RV & 5'-GAGAGCGGCCGCTCATATTTTGCTCGTATTCC-3' \\
JS_gatCmut2_FW & 5'-GTTGCCAAAGTGGGAGACCTGCAAACCCTGGGTC-3' \\
JS_gatCmut2_RV & 5'-GACCCAGGGTTTGCAGGTCTCCCACTTTGGCAAC-3' \\
JS_XInDp_FW & 5'-AGTCAATCGGTCATTCTCCG-3' \\
JS_gatA_RV & 5'-CTCTTGGAGGACGCAAAACC-3' \\
JS_gatB_R & V 5'-CATGGAAGCGGTGATCTAGG-3' \\
\hline
\end{tabular}

\section{Analysis of $\boldsymbol{A}$. niger transformants}

Screening of randomly picked transformants was done by PCR on genomic DNA isolates. For that, fresh mycelium was disrupted using MP lysing matrix $\mathrm{C}$ tubes and $400 \mu \mathrm{l}$ DNA extraction buffer $(0.1 \mathrm{M}$ Tris $\mathrm{HCl} \mathrm{pH} 8.0,1.2 \mathrm{M} \mathrm{NaCl}, 5 \mathrm{mM}$ EDTA). DNA was extracted using phenol-chloroform extraction. The pellet was washed with $70 \%$ cold ethanol, air-dried and re-suspended in $50 \mu 1 \mathrm{MQ}$ water. The presence of An14g04280, An03g01620 and An07g00780 expression constructs was confirmed by PCR using the JS_XlnDp_FW primer, binding to the promoter region of the construct, and $\overline{\mathrm{JS}}$ gat $A \mathrm{RV}$, JS gat $B \mathrm{RV}$ or JS_gatC_RV specifically binding to the complementary strand in the corresponding coding region. Transformants with confirmed gene constructs were re-plated on complete medium. Spores are harvested after 4 days of growth at $30^{\circ} \mathrm{C}$.

\section{Growth in shake flasks}

Strains were grown in duplicate in $200 \mathrm{~mL}$ PM medium $\left(1.2 \mathrm{~g} \mathrm{NaNO}_{3}, 0.5 \mathrm{~g}\right.$ $\mathrm{KH}_{2} \mathrm{PO}_{4}, 0.2 \mathrm{~g} \mathrm{MgSO}_{4} 7 \mathrm{H}_{2} \mathrm{O}, 0.5 \mathrm{~g}$ Yeast extract and $40 \mu \mathrm{g}$ Vishniac per liter) [9] with $91 \mathrm{mM}$ sorbitol and $9 \mathrm{mM}$ D-galacturonic acid or $100 \mathrm{mM}$ of the synthetic cell wall hydrolysate $(\mathrm{SCH})$ [13] (Table 3) as a carbon source in $1 \mathrm{~L}$ flasks. For inoculation, a final concentration of $1 \bullet 10_{6}$ spores per $\mathrm{mL}$ was used. Induction of the $x \ln D$ promoter with $10 \mathrm{mM}$ D-xylose was done at $\mathrm{t}=0,18$ hours 
after inoculation [12]. Transformants were grown for 5 days at $30^{\circ} \mathrm{C}$ and 250 rpm. Medium samples were taken by filtration of $2 \mathrm{ml}$ of the culture broth through a 5-micron pore size nylon filter. Biomass samples were obtained by washing of the retentate with demineralized water and flash freezing using liquid nitrogen. The samples were taken at $\mathrm{T}=-18 \mathrm{~h}, 0 \mathrm{~h}, 6 \mathrm{~h}, 30 \mathrm{~h}, 54 \mathrm{~h}$ for the first growth experiment on D-galacturonic acid. For the second D-galacturonic acid growth experiment, samples were taken at $\mathrm{T}=-18 \mathrm{~h}$, each hour from $\mathrm{T}=0 \mathrm{~h}$ to $\mathrm{T}=10 \mathrm{~h}, 24 \mathrm{~h}, 30 \mathrm{~h}, 54 \mathrm{~h}$ and $78 \mathrm{~h}$. For the growth experiment with $\mathrm{SCH}$ as a carbon source, samples were taken from $\mathrm{T}=0 \mathrm{~h}$ with steps of 24 and 6 hours: $\mathrm{T}$ $=0 \mathrm{~h}, \mathrm{~T}=24 \mathrm{~h}, \mathrm{~T}=30 \mathrm{~h}, \mathrm{~T}=48 \mathrm{~h}, \mathrm{~T}=54 \mathrm{~h}$.

Table 3 Composition of cell wall hydrolysate

\begin{tabular}{ll}
\hline Compound \% & Concentration (g/l) \\
\hline D-glucose; 65\% & 13 \\
D-xylose; 20\% & 4 \\
D-galacturonic acid; 10\% & 2 \\
L-arabinose; 3\% & 0.6 \\
D-galactose; 1\% & 0.2 \\
D-mannose; 1\% & 0.2 \\
\hline
\end{tabular}

\section{Growth in fermentors}

Strains were grown in duplicate in $500 \mathrm{~mL}$ PM medium [9] with $100 \mathrm{mM}$ sorbitol as a carbon source in $1 \mathrm{~L}$ flasks. For inoculation, a final concentration of $1 \bullet 10_{6}$ spores per $\mathrm{mL}$ was used. Cultures were incubated for 18 hours at $30^{\circ} \mathrm{C}$ with 250 rpm orbital shaking. Mycelium was aseptically harvested by filtering over a sterile nylon cloth, washed with PM without carbon source and transferred to 1 $\mathrm{L}$ fermentors. Fermentors contained $750 \mathrm{ml} \mathrm{PM}$ with $50 \mathrm{mM}$ D-xylose and 50 $\mathrm{mM}$ D-galacturonic acid, adjusted to $\mathrm{pH} 6$. Air was flushed at a stirrer speed of $600 \mathrm{rpm}$. Medium and biomass samples were taken as previously described, with an additional sampling of mycelium from $10 \mathrm{ml}$ broth volume for enzymatic assays. Carbon dioxide and oxygen concentrations in off-gas were measured as well as $\mathrm{pH}$.

\section{HPLC analysis}

High-pressure liquid chromatography (HPLC) was used to determine the extracellular concentrations of D-glucose, L-sorbitol, D-xylose, citric acid and D-galacturonic acid in the culture broth samples. A Shodex KC-811 column was used at $30^{\circ} \mathrm{C}$ that was eluted with $0.01 \mathrm{~N} \mathrm{H}_{2} \mathrm{SO}_{4}$ at a flow rate of $0.8 \mathrm{~mL}$ min-1. A refractive index detector (Spectrasystem RI-150, sample frequency $5.00032 \mathrm{~Hz}$ ) and a UV-VIS detector (Spectrasystem UV1000, $\lambda=210 \mathrm{~nm}$ ) were used for 
detection of the eluting compounds. Crotonate at a concentration of $6 \mathrm{mM}$ was used as an internal standard.

\section{Enzyme assay}

To determine the activity of the D-galacturonic acid metabolism, the first step in the enzymatic conversion, D-galacturonic acid to L-galactonic acid, was measured. D-galacturonic acid reductase assay based on conversion of NADPH to NADP+, previously described by Kuorelahti et al., was applied [26]. $20 \mathrm{mg}$ of frozen mycelium was added to $400 \mu \mathrm{l}$ of extraction buffer $(100 \mathrm{mM}$ phosphate buffer $\mathrm{pH}$ 7.0, $0.1 \mathrm{mM}$ EDTA, $1 \mathrm{mM}$ DTT and fungal protease inhibitor cocktail) in lysing matrix $\mathrm{C}$ tubes (MP biomedicals) and disrupted at level 6 for 30 seconds in a bead beater (MP fastprep-24). Cell debris was removed by centrifugation and supernatant was used as cell extract. $25 \mu \mathrm{l}$ of cell extract was added to $200 \mu 1$ of assay buffer (100 mM sodium phosphate buffer $\mathrm{pH} 7.0,0.25 \mathrm{mM} \mathrm{NADPH})$ and reaction was started by the addition of $25 \mu 1$ of substrate $(1 \mathrm{M} \mathrm{D}$-galacturonic acid pH 6). Decrease of absorbance at $340 \mathrm{~nm}$ was measured on a plate reader (Biotek Synergy) in parallel with sample controls, for which $25 \mu 1$ of buffer was added. Background activity of sample control without D-galacturonic acid was subtracted from sample measurements with D-galacturonic acid. Protein content was determined by photometric assay [27]. 


\section{References}

1. Howard RL, Abotsi E, Van Rensburg ELJ, Howard S. Lignocellulose biotechnology: issues of bioconversion and enzyme production. African J Biotechnol. Academic Journals (Kenya); 2003;2: 602-619.

2. Willke T, Vorlop K-D. Biotechnological production of itaconic acid. Appl Microbiol Biotechnol. Springer; 2001;56: 289-295.

3. van Dijck PWM, Selten GCM, Hempenius RA. On the safety of a new generation of DSM Aspergillus niger enzyme production strains. Regul Toxicol Pharmacol. Elsevier; 2003;38: 27-35.

4. Ángel Siles López J, Li Q, Thompson IP. Biorefinery of waste orange peel. Crit Rev Biotechnol. Taylor \& Francis; 2010;30: 63-69.

5. Micard V, Renard C, Thibault J-F. Enzymatic saccharification of sugar-beet pulp. Enzyme Microb Technol. Elsevier; 1996;19: 162-170.

6. van den Brink J, de Vries RP. Fungal enzyme sets for plant polysaccharide degradation. Appl Microbiol Biotechnol. Springer; 2011;91: 1477-1492.

7. Gielkens MMC, Dekkers E, Visser J, de Graaff LH. Two cellobiohydrolaseencoding genes from Aspergillus niger require D-xylose and the xylanolytic transcriptional activator XlnR for their expression. Appl Environ Microbiol. Am Soc Microbiol; 1999;65: 4340-4345.

8. de Vries RP, Visser J, de Graaff LH. CreA modulates the XlnR-induced expression on xylose of Aspergillus niger genes involved in xylan degradation. Res Microbiol. Elsevier; 1999;150: 281-285.

9. Ruijter GJG, van de Vondervoort PJI, Visser J. Oxalic acid production by Aspergillus niger: an oxalate-non-producing mutant produces citric acid at $\mathrm{pH} 5$ and in the presence of manganese. Microbiology. Microbiology Society; 1999;145: 2569-2576.

10. Peij NNME, Gielkens MMC, Vries RP, Visser J, Graaff LH. The transcriptional activator $\mathrm{XlnR}$ regulates both xylanolytic and endoglucanase gene expression in Aspergillus niger. Appl Env Microbiol. 1998;64.

11. Martens-Uzunova ES, Schaap PJ. An evolutionary conserved D-galacturonic acid metabolic pathway operates across filamentous fungi capable of pectin degradation. Fungal Genet Biol. Elsevier; 2008;45: 1449-1457.

12. van der Veen D, Oliveira JM, Van den Berg WAM, de Graaff LH. Analysis of variance components reveals the contribution of sample processing to transcript variation. Appl Environ Microbiol. Am Soc Microbiol; 2009; 75: 2414 2422.

13. Carpita NC, Gibeaut DM. Structural models of primary cell walls in flowering plants: consistency of molecular structure with the physical properties of the walls during growth. Plant J. Wiley Online Library; 1993;3: 1-30. 
14. Carlsen M, Nielsen J. Influence of carbon source on $\alpha$-amylase production by Aspergillus oryzae. Appl Microbiol Biotechnol. Springer; 2001;57: 346-349.

15. Benz JP, Protzko RJ, Andrich JMS, Bauer S, Dueber JE, Somerville CR. Identification and characterization of a galacturonic acid transporter from Neurospora crassa and its application for Saccharomyces cerevisiae fermentation processes. Biotechnol Biofuels. BioMed Central; 2014;7: 1.

16. Zhang L, Hua C, Stassen JHM, Chatterjee S, Cornelissen M, van Kan JAL. Genome-wide analysis of pectate-induced gene expression in Botrytis cinerea: Identification and functional analysis of putative D-galacturonate transporters. Fungal Genet Biol. Elsevier; 2014;72: 182-191.

17. Overbeek R, Fonstein M, D'souza M, Pusch GD, Maltsev N. The use of gene clusters to infer functional coupling. Proc Natl Acad Sci. National Acad Sciences; 1999;96: 2896-2901.

18. Larkin MA, Blackshields G, Brown NP, Chenna R, McGettigan PA, McWilliam H, et al. Clustal W and Clustal X version 2.0. bioinformatics. Oxford Univ Press; 2007;23: 2947-2948.

19. Viklund H, Elofsson A. Best $\alpha$-helical transmembrane protein topology predictions are achieved using hidden Markov models and evolutionary information. Protein Sci. Wiley Online Library; 2004;13: 1908-1917.

20. Yan N. Structural advances for the major facilitator superfamily (MFS) transporters. Trends Biochem Sci. Elsevier Ltd; 2013;38: 151-159. doi:10.1016/j.tibs.2013.01.003

21. Mach-Aigner AR, Omony J, Jovanovic B, van Boxtel AJB, de Graaff LH. dXylose Concentration-Dependent Hydrolase Expression Profiles and the Function of CreA and XlnR in Aspergillus niger. Appl Environ Microbiol . 2012;78: 3145-3155. doi:10.1128/AEM.07772-11

22. Kuivanen J, Mojzita D, Wang Y, Hilditch S, Penttilä M, Richard P, et al. Engineering filamentous fungi for conversion of D-galacturonic acid to Lgalactonic acid. Appl Environ Microbiol. Am Soc Microbiol; 2012;78: 86768683.

23. Arnaud MB, Cerqueira GC, Inglis DO, Skrzypek MS, Binkley J, Chibucos $\mathrm{MC}$, et al. The Aspergillus Genome Database (AspGD): recent developments in comprehensive multispecies curation, comparative genomics and community resources. Nucleic Acids Res. Oxford Univ Press; 2012;40: D653-D659.

24. Pfaffl MW. A new mathematical model for relative quantification in realtime RT-PCR. Nucleic Acids Res. Oxford Univ Press; 2001;29: e45-e45.

25. Kusters-van Someren MA, Harmsen JAM, Kester HCM, Visser J. Structure of the Aspergillus niger pelA gene and its expression in Aspergillus niger and Aspergillus nidulans. Curr Genet. Springer; 1991;20: 293-299. 
26. Kuorelahti S, Kalkkinen N, Penttilä M, Londesborough J, Richard P. Identification in the mold Hypocrea jecorina of the first fungal D-galacturonic acid reductase. Biochemistry. ACS Publications; 2005;44: 11234-11240.

27. Peterson GL. A simplification of the protein assay method of Lowry et al. which is more generally applicable. Anal Biochem. Elsevier; 1977;83: 346-356. 


\section{Chapter 6}

\section{Identification of a novel L-rhamnose uptake transporter in the filamentous fungus Aspergillus niger}

Jasper Sloothaak, Dorett I. Odoni, Vitor A. P. Martins dos Santos, Peter J. Schaap and Juan Antonio Tamayo-Ramos

PLOS Genetics (2016) Vol 12 


\title{
Identification of a novel L-rhamnose uptake transporter in the filamentous fungus Aspergillus niger
}

\begin{abstract}
The study of plant biomass utilization by fungi is a research field of great interest due to its many implications in ecology, agriculture and biotechnology. Most of the efforts done to increase the understanding of the use of plant cell walls by fungi have been focused on the degradation of cellulose and hemicellulose, and transport and metabolism of their constituent monosaccharides. Pectin is another important constituent of plant cell walls, but has received less attention. In relation to the uptake of pectic building blocks, fungal transporters for the uptake of galacturonic acid recently have been reported in Aspergillus niger and Neurospora crassa. However, not a single L-rhamnose (6-deoxy-L-mannose) transporter has been identified yet in fungi or in other eukaryotic organisms. Lrhamnose is a deoxy-sugar present in plant cell wall pectic polysaccharides (mainly rhamnogalacturonan I and rhamnogalacturonan II), but is also found in diverse plant secondary metabolites (e.g. anthocyanins, flavonoids and triterpenoids), in the green seaweed sulfated polysaccharide ulvan, and in glycan structures from viruses and bacteria. Here, a comparative plasmalemma proteomic analysis was used to identify candidate L-rhamnose transporters in $A$. niger. Further analysis was focused on protein ID 1119135 (RhtA) (JGI A. niger ATCC 1015 genome database). RhtA was classified as a Family 7 Fucose: H+ Symporter (FHS) within the Major Facilitator Superfamily. Family 7 currently includes exclusively bacterial transporters able to use different sugars. Strong indications for its role in L-rhamnose transport were obtained by functional complementation of the Saccharomyces cerevisiae EBY.VW.4000 strain in growth studies with a range of potential substrates. Biochemical analysis using $\mathrm{L}-[3 \mathrm{H}(\mathrm{G})]$-rhamnose confirmed that RhtA is a L-rhamnose transporter. The RhtA gene is located in tandem with a hypothetical alpha-L-rhamnosidase gene $(r h a B)$. Transcriptional analysis of $r h t A$ and $r h a B$ confirmed that both genes have a coordinated expression, being strongly and specifically induced by Lrhamnose, and controlled by RhaR, a transcriptional regulator involved in the release and catabolism of the methyl-pentose. RhtA is the first eukaryotic Lrhamnose transporter identified and functionally validated to date.
\end{abstract}




\section{Background}

Organic carbon utilization by fungi is a biological process of great interest with many implications in ecology, agriculture and biotechnology. Their ubiquity and their ability to mobilize and metabolize a large variety of nutrients make fungi crucial players in the biogeochemical cycling of carbon in nature, in mutualistic symbiotic relationships with plants, and in pathogenic processes [1]. Their physiological resources for carbon utilization and biotransformation have also enhanced their relevance in the fields of (food) fermentation, bioindustrial chemistry and pharmacy, as they can be exploited for the production of enzymes, chemicals and other components of interest [2]. Thus, important efforts for the understanding of carbon utilization by fungi have been done, historically on those organisms that are genetically amenable, or have a direct impact on human affairs. In this sense, filamentous fungi from the Aspergillus genera, which include model species, species relevant for industrial applications, and human, animal and plant pathogens, have been deeply studied. In particular, the fungus Aspergillus niger has a versatile system for the degradation of the major plant cell wall polysaccharides: cellulose, hemicellulose and pectin, and due to its high enzyme secretory capacity is one of the main industrial producers of commercial enzymes for plant biomass conversion $[3,4]$. A. niger is able to synthetize an abundance of extracellular enzymes for lignocellulose depolymerization, and the encoding genes as well as the regulatory circuits that control their expression have been identified and characterized in detail [5-9]. Pectin utilization by fungi has received less attention, probably due to its structural complexity and to the nature of its polysaccharides. Pectin is composed of mainly D-galacturonic acid (approx. 65\%), L-rhamnose, and branched with heterogeneous oligosaccharides. [10]. L-rhamnose is specifically found to be enriched in the pectic polysaccharide fractions rhamnogalacturonan I and II. The pectin depolymerization enzyme network has been partly identified and characterized in several fungi [11-13], the catabolic pathways for the conversion of D-galacturonic acid and L-rhamnose have been genetically and biochemically characterized [14-17], and recently the transcription factors responsible for L-rhamnose and D-galacturonic acid utilization, RhaR and GaaR, have been identified [18,19]. Regarding the uptake of the specific pectin components, transporters responsible for the uptake of Dgalacturonic acid have been reported in A. niger and Neurospora crassa [20,21]. However, not a single L-rhamnose transporter has been identified in fungi, or in any other eukaryotic organism. Besides its structural role in pectin, L-rhamnose is part of plant glycoproteins and secondary metabolites too, it is an important component of the green seaweed sulfated polysaccharide ulvan, and it is also present in glycan structures from virus and bacteria [22-24]. 


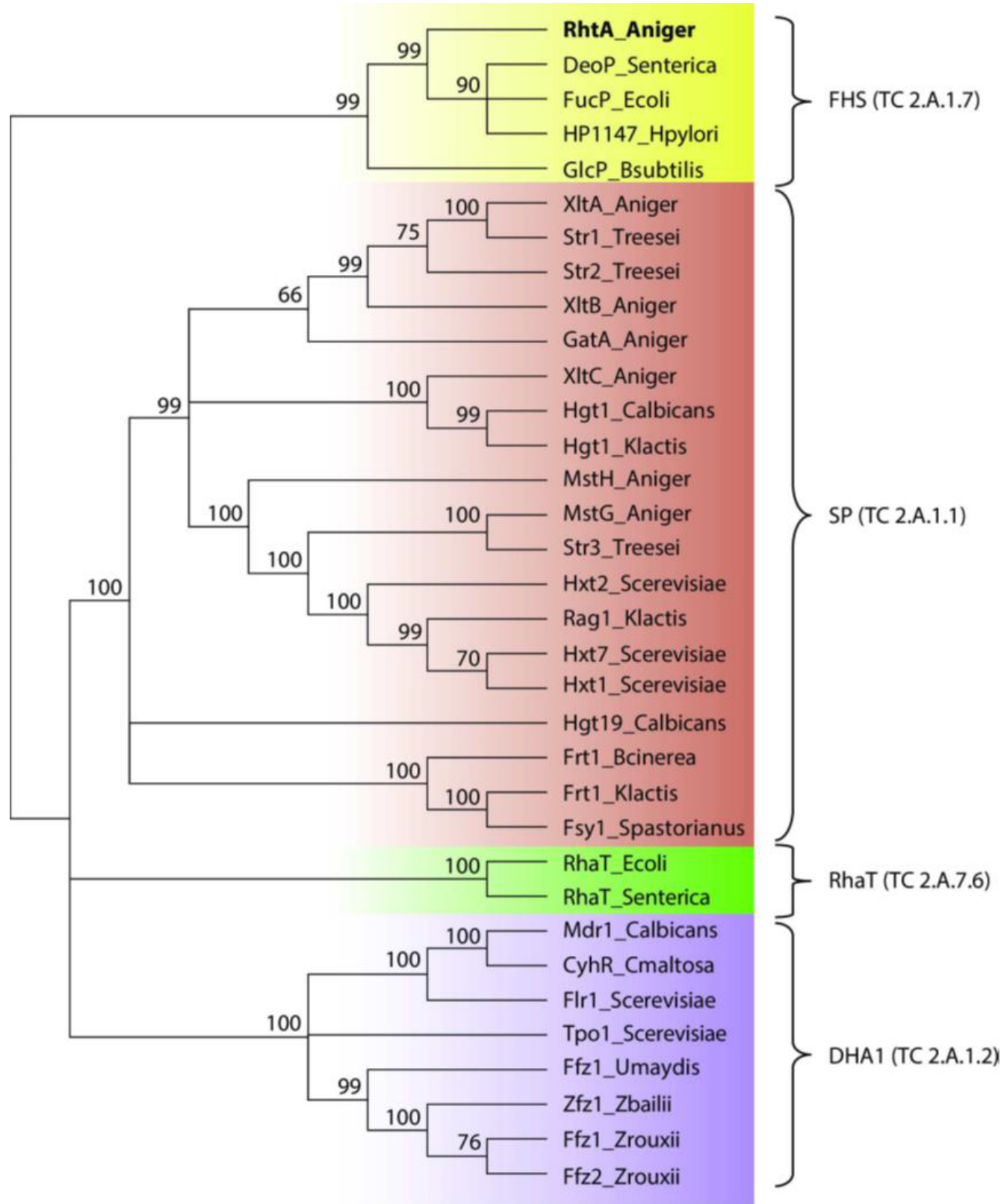

Figure 1 Classification of $A$. niger RhtA. Sequences of biochemically characterized sugar transporters were collected and a multiple sequence alignment was created using Praline alignment suite, which takes secondary structure predictions into account [83]. A neighbour-joining tree was then generated with 1000 bootstrap replicates 
In prokaryotes, L-rhamnose uptake occurs via transporters from the RhaT family (2.A.7.6), which belongs to the drug/metabolite transporter superfamily (2.A.7) [25], but eukaryotic transporters belonging to the RhaT family have not been reported. Recently, an intracellular UDP-rhamnose/UDP-galactose transporter (nucleotide sugar transporter; NST), also member of the DMT superfamily, and located on the Golgi apparatus of Arabidopsis thaliana, was identified [26]. However NSTs are involved in transport processes related to the biosynthesis of plant cell wall components, and glycan structures, not found in eukaryotic plasma membranes, and not related to the uptake of sugars present in the environment. Monosaccharide transport by fungi has been investigated mainly inSaccharomyces cerevisiae in which transport of simple sugars is mediated only through facilitated diffusion by transporters from the sugar porter family, the largest within the major facilitator superfamily (MFS) [27]. The use of yeast monosaccharide transporter null mutants allowed for individual characterization of the individual transporters responsible for the uptake of D-glucose, D-fructose, D-mannose and Dgalactose in yeast [27-29]. Yeast transporter null mutants were subsequently also used for the functional characterization of sugar porters from other fungal species [30-35]. The analyzes of the global transcriptomic and proteomic responses of fungi to a variety of specific culture conditions are useful approaches to get insights in the specific structural and regulatory elements required for the utilization of specific carbon sources. In order to identify Lrhamnose transporter candidates, in this study a comparative plasmalemma proteome analysis was performed. The identification and functional validation of a eukaryotic L-rhamnose transporter is reported for the first time. 
Table 1 Relative abundance of MFS transporter proteins detected in different $A$. niger growth conditions

\begin{tabular}{|l|l|l|l|l|}
\hline \multirow{2}{*}{ Prot ID } & \multicolumn{4}{|l|}{ Relative abundance \pm sd (\%) $\mathbf{x} 100$} \\
\cline { 2 - 5 } & L-arabinose & D-mannose & L-rhamnose & $\begin{array}{l}\text { D-sorbitol + D- } \\
\text { xylose }\end{array}$ \\
\hline 1089440 & n.d. & n.d. & $1.91 \pm 0.98$ & n.d. \\
\hline 1096151 & n.d. & n.d. & $4.20 \pm 2.40$ & n.d. \\
\hline 1101809 & $5.12 \pm 1.73$ & $1.60 \pm 0.08$ & $7.94 \pm 0.90$ & $2.95 \pm 0.24$ \\
\hline 1105147 & $6.2 \pm 0.21$ & $8.13 \pm 0.82$ & $11.78 \pm 0.22$ & $6.78 \pm 0.44$ \\
\hline 1105500 & $0.54 \pm 0.2$ & n.d. & n.d. & n.d. \\
\hline 1111630 & $0.49 \pm 0.1$ & $1.71 \pm 0.15$ & $1.31 \pm 0.14$ & $1.38 \pm 0.45$ \\
\hline $1119135 ;($ RhtA) & n.d. & n.d. & $10.94 \pm 4.21$ & n.d. \\
\hline 1121621 & $3.64 \pm 1.31$ & $5.90 \pm 0.73$ & n.d. & $4.96 \pm 0.48$ \\
\hline 1122202 & $0.63 \pm 0.2$ & $0.92 \pm 0.05$ & $0.46 \pm 0.19$ & $0.28 \pm 0.01$ \\
\hline 1125086 & $37.93 \pm 5.96$ & n.d. & n.d. & n.d. \\
\hline 1128338 & n.d. & $4.98 \pm 1.11$ & n.d. & $2.03 \pm 0.07$ \\
\hline 1129336 & n.d. & $0.39 \pm 0.08$ & n.d. & $0.26 \pm 0.01$ \\
\hline 1142034 & n.d. & n.d. & $2.26 \pm 0.04$ & n.d. \\
\hline 1142882 & $2.95 \pm 0.32$ & $2.30 \pm 0.56$ & $4.40 \pm 0.11$ & $2.21 \pm 0.50$ \\
\hline 1143191 & n.d. & n.d. & $7.04 \pm 0.97$ & n.d. \\
\hline 1143598 & $3.34 \pm 0.89$ & $4.59 \pm 2.03$ & $6.51 \pm 5.28$ & $8.51 \pm 0.12$ \\
\hline 1144375 & $5.8 \pm 2.89$ & $0.72 \pm 0.23$ & $15.25 \pm 3.84$ & n.d. \\
\hline 1144791 & $16.06 \pm 4.06$ & n.d. & $17.75 \pm 2.24$ & $6.20 \pm 1.05$ \\
\hline 1147409 & n.d. & n.d. & $0.78 \pm 0.44$ & n.d. \\
\hline 1156895 & n.d. & n.d. & $10.92 \pm 0.57$ & n.d. \\
\hline 1160647 & $2.47 \pm 1.61$ & n.d. & n.d. & n.d. \\
\hline $1164538 ;$ & $0.55 \pm 0.11$ & $1.30 \pm 0.12$ & $1.03 \pm 0.24$ & $0.95 \pm 0.01$ \\
$1188786 *$ & & & & \\
\hline 1165706 & n.d. & n.d. & n.d. & $0.06 \pm 0.05$ \\
\hline 1167504 & $1.07 \pm 0.09$ & $0.39 \pm 0.12$ & n.d. & n.d. \\
\hline 1169204 & $0.54 \pm 0.13$ & n.d. & n.d. & $5.07 \pm 0.95$ \\
\hline 1178623 & $7.53 \pm 2.54$ & $5.20 \pm 0.00$ & $5.64 \pm 4.12$ & n.d. \\
\hline 1180703 & n.d. & n.d. & $2.61 \pm 0.25$ & n.d. \\
\hline 1188093 & $0.85 \pm 0.08$ & $0.96 \pm 0.15$ & $1.59 \pm 0.15$ & $0.78 \pm 0.16$ \\
\hline 1188840 & n.d. & $0.60 \pm 0.24$ & n.d. & n.d. \\
\hline 1189214 & $35.93 \pm 0.54$ & n.d. & $2.87 \pm 1.02$ & n.d. \\
\hline & & & & \\
\hline & & & & \\
\hline & & & & \\
\hline
\end{tabular}




\section{Results}

Comparative plasmalemma proteome analysis for the identification of $\mathrm{A}$. niger L-rhamnose transporter candidates

A recently developed approach for the study of the A. niger transportome through its plasma membrane proteomic analysis was used in order to perform a differential protein expression analysis of $A$. niger major facilitator superfamily (MFS) transporters. The approach is based on a LC-MS/MS analysis of plasmalemma enriched cellular fractions, and the method was applied to study the $A$. niger response to different D-glucose concentrations [36]. In the present study, a similar experimental set-up was used, but here the $A$. niger response to the presence of D-mannose, L-arabinose, D-xylose and L-rhamnose was studied. Mycelium of $A$. niger $\mathrm{N} 400$ was pre-grown for $18 \mathrm{~h}$ in minimal medium supplemented with $100 \mathrm{mM}$ D-sorbitol as sole carbon source and equal amounts of biomass was transferred to controlled fermenters containing minimal medium with the following carbon source compositions: D-sorbitol $100 \mathrm{mM}$ plus $0.1 \mathrm{mM}$ D-xylose, $5 \mathrm{mM}$ D-mannose, $5 \mathrm{mM}$ L-arabinose or $5 \mathrm{mM}$ L-rhamnose. The initial $\mathrm{pH}$ of these cultures was set at $\mathrm{pH} 4.0$ and controlled at a lower limit of $\mathrm{pH} 3.5$. Two hours after inoculation, mycelium was taken and cellular fractions enriched for plasma membranes were obtained as previously reported (see Materials and Methods and [36] for details). High-resolution analysis of the sugar content in the culture medium at the time of sampling showed that in all four conditions the respective sugars were being consumed (S1 Fig), so the presence of active transporters for the different monosaccharides could be expected. For each culture condition peptide MS/MS spectra, obtained from the LC-MS/MS analysis of the enriched plasmalemma fractions, were processed as described in the Materials and Methods section. In total, 958 proteins were identified, of which 510 were present in all four conditions, while 41, 40, 65, and 43 proteins were present exclusively in the L-arabinose, D-mannose, L-rhamnose, and D-xylose conditions, respectively (S1 Dataset). The aim of this study was to identify specific L-rhamnose transporters, so the comparative proteome analysis was focused on the identification and abundance analysis of candidate sugar porters. A total of 15 MFS transporters were identified in the presence of D-xylose, 16 in the presence of D-mannose, 19 in the presence of L-arabinose and 21 in the presence of L-rhamnose, of which 8 were shown to be exclusively detected in the L-rhamnose condition (Table 1). From these eight, a subgroup of five having strain ATCC 1015 [3] protein ID 1096151, 1119135, 1142034, 1147409 and 1156895 respectively were also absent in a previously generated dataset, where the $A$. niger plasmalemma proteome response to high and low concentrations of 
D-glucose was studied [36]. In summary, by analyzing the $A$. niger plasma membrane proteome response to seven different carbon source compositions (Dsorbitol $100 \mathrm{mM}$, D-sorbitol $100 \mathrm{mM}$ plus D-glucose $1 \mathrm{mM}$, D-sorbitol $100 \mathrm{mM}$ plus D-glucose $60 \mathrm{mM}$, D-sorbitol $100 \mathrm{mM}$ plus D-xylose $0.1 \mathrm{mM}$, L-arabinose $5 \mathrm{mM}$, D-mannose $5 \mathrm{mM}$, and L-rhamnose $5 \mathrm{mM}$ ) five putative transport proteins could be identified that were present only in the presence of L-rhamnose, which strongly suggested involvement of one or more of these transporters in the uptake of L-rhamnose. A detailed protein sequence analysis of these transporters and of their encoding genes highlighted some interesting features of protein ID 1119135, hereinafter referred to as RhtA for Rhamnose transporter A. Domain analysis of the transporters revealed that RhtA was exceptional because it possessed a L-fucose permease domain structure (FucP; COG0738/ IPR005275). FucP domain transporters have been shown to be able to use different deoxy sugars as substrate, such as L-fucose, 2-deoxy-D-ribose and 2-deoxy-D-glucose, but also monosaccharides such as L-arabinose, D-galactose, D-glucose and Dmannose [38-40]. To our knowledge transporters with a FucP domain structure have only been characterized from bacteria. However, the particular domain structure is well represented throughout the fungal kingdom. Through a Bidirectional Best Hit (BBH) BLAST analysis, putative RhtA orthologs were inferred. Homologous sequences were found throughout different fungal orders, but only $5 \mathrm{BBH}$ 's were identified when analyzing the available genomes of Aspergillus spp. (S1 Table). A domain based classification of RhtA, using amino acid sequences of 27 functionally validated fungal MFS sugar transporters, 2 bacterial L-rhamnose transporters, and 4 characterized bacterial FucP domain symporters as input, grouped the RhtA transporter with the latter group (Fig 1). These transporters belong to the Fucose:H1 symporter (FHS) family (TC 2.A.1.7) within the Major Facilitator Superfamily [41] (Fig 1). The domain architecture COG0738 is defined by 13 reference sequences, of which Bacillus subtilis GlcP, Escherichia coli FucP and Helicobacter pylori HP1174 [38,39,42] have been characterized, while DeoP from Salmonella enterica is probably a 2-deoxy-Dribose permease [40]. E. coli FucP transports L-fucose, L-arabinose and Dgalactose but it is not able to transport L-rhamnose, $B$. subtilis GlcP has high affinity for D-glucose and D-mannose, and H. pylori HP1174 is able to use Dglucose, D-galactose, D-mannose and 2-deoxy-D-glucose as substrates. L-fucose and L-rhamnose are structurally related, as both of them are methyl pentoses, however there have been no reports on the ability of FHS symporters to use Lrhamnose as substrate, for which specific transporters from the L-rhamnose proton symport family (RhaT; cl05728/IPR004673) have been described in bacteria [43]. Characterized bacterial L-rhamnose transporters from the L- 
rhamnose transporter family (RhaT) (TC 2.A.7.6) clustered together in a separate subgroup in the phylogenetic tree (Fig 1). This was expected because they are not related to the MFS (2.A.1) and have no similarities with proteins from this superfamily [44]. The remaining fungal functionally validated sugar transporters are clustered in two additional subgroups corresponding to other MFS families: the sugar porter (SP) family (TC 2.A.1.1) and the drug:H1 antiporter-1 (12 spanner) (DHA1) (TC 2.A.1.2) (Fig 1). Analysis of the genomic locus of the RhtA encoding gene revealed directly upstream of $r h t A$ an ORF coding for a hypothetical secreted glycoside hydrolase, hereinafter referred to $r h a B$, from the GH78 family (Protein ID 131668) which only includes $\alpha$-L-rhamnosidases. The hypothetical RhaB is a 832 amino acid protein with low similarity to functionally validated $\alpha$-L-rhamnosidases. However, its amino acid number is in the range of RhaE from $A$. nidulans (861 aa), and many of the characterized bacterial rhamnosidases [13]. The rhaB-rhtA tandem localization, which could conform a L-rhamnose uptake system in $A$. niger, was found to be present as well in Aspergillus luchuensis and Aspergillus kawachii, which have been reported to be the same species [45], and are closely related to A. niger. Taking the abovedescribed findings into account, RhtA was considered a strong candidate to be a transporter specific for L-rhamnose. In order to validate this hypothesis, and to unravel the function of this eukaryotic transporter with a FucP domain signature, RhtA was selected for functional validation and characterization in the present study.

\section{Functional validation of the $A$. niger sugar transporter RhtA}

In order to test the functionality of the RhtA transporter, the engineered $S$. cerevisiae strain EBY.VW4000, a monosaccharide transporter null mutant, was chosen as host for the heterologous expression of the rhtA coding gene. $S$. cerevisiae is not able to use L-rhamnose as a carbon source, so a direct functional complementation approach based on the use of this deoxy sugar could not be performed with this strain. Despite this, and as discussed below, heterologous expression of the transporter in this genetic background gave clear insights about the possible role of RhtA on L-rhamnose transport. The yeast strain was transformed with the $2 \mu$ expression plasmid p426HXT7-6His-rhtA, containing the gene's cDNA under control of the constitutive promoter $\mathrm{HXT}_{\mathrm{p}}$ and the terminator $\mathrm{CYCl}_{\mathrm{t}}$. Single colony transformants were isolated from minimal medium agar plates containing $2 \%(\mathrm{w} / \mathrm{v})$ maltose and the ability of $r h t A$ to restore growth of the EBY.VW4000 transformant strain in the presence of different monosaccharides was studied. Ten-fold serial dilutions of logarithmically growing cells from at least three different transformants expressing $r h t A$ were 


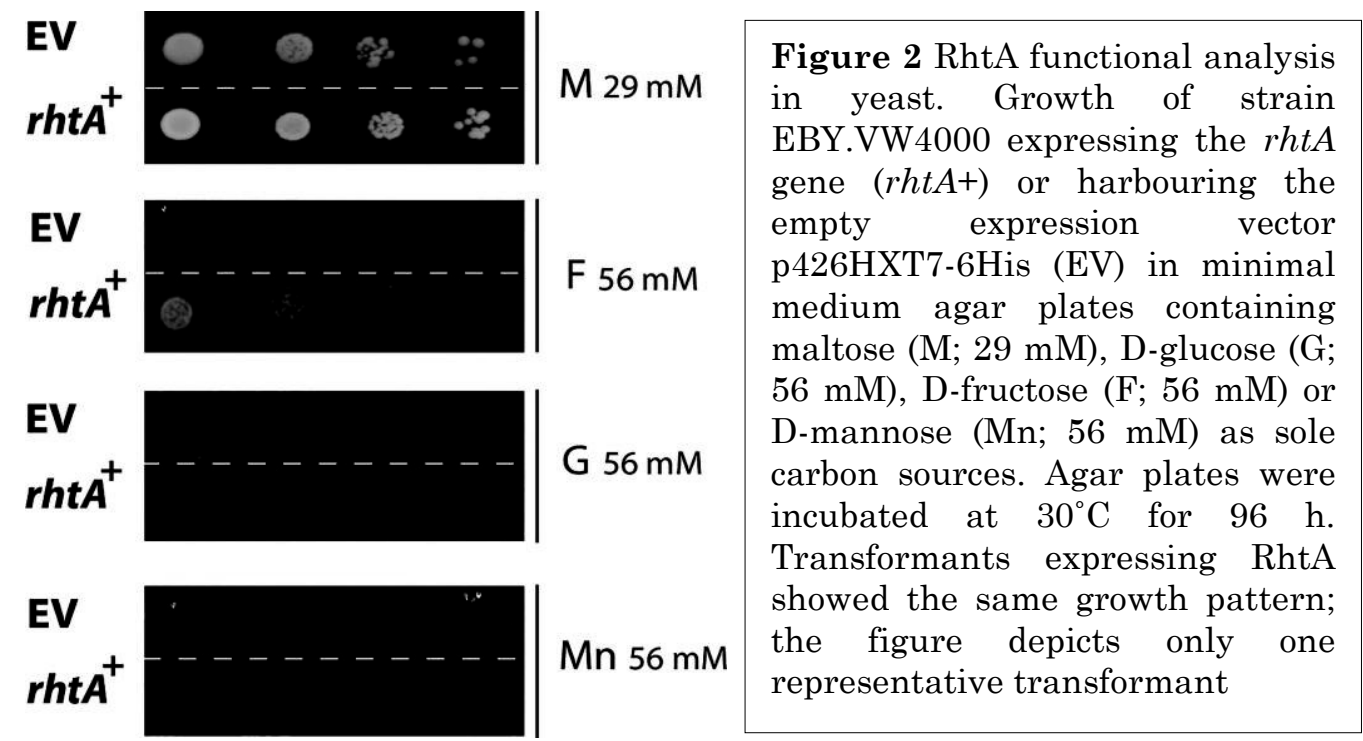

spotted on different minimal medium plates supplemented with $1 \%(\mathrm{w} / \mathrm{v})$ of the following carbon sources: D-glucose $(\mathrm{G} ; 56 \mathrm{mM})$, D-fructose $(\mathrm{F} ; 56 \mathrm{mM})$, Dmannose $(\mathrm{Mn} ; 56 \mathrm{mM})$ and maltose $(\mathrm{M} ; 29 \mathrm{mM})$. Yeast rhtA transformants showed an ability to restore growth on D-fructose, albeit at a slow pace, but were not able to restore growth on D-glucose and D-mannose (Fig 2). This result indicated that RhtA was functional as a transporter in $S$. cerevisiae, but as expected none of the substrates tested seemed to be ideal for this transporter. The fact that D-fructose was used as a substrate by RhtA allowed us to perform sugar competition assays on plate, which gave more insights in possible additional substrates for this transporter. Hence, the ability of an $r h t A$ transformant to grow in the presence of D-fructose $(\mathrm{F} ; 28 \mathrm{mM})$ was compared to its ability to grow in plates containing D-fructose $(\mathrm{F} ; 28 \mathrm{mM})$ mixed with either D-glucose $(\mathrm{G} ; 56 \mathrm{mM}$ or $5.6 \mathrm{mM})$, D-xylose (X; $66 \mathrm{mM}$ or $6.6 \mathrm{mM})$, L-arabinose (A; $66 \mathrm{mM}$ or 6.6 $\mathrm{mM}$ ), D-sorbitol (S; $55 \mathrm{mM}$ or $5.5 \mathrm{mM}$ ) or L-rhamnose (R; $61 \mathrm{mM}$ or $6.1 \mathrm{mM})$. The rhtA transformant strain was able to grow in the presence of most of the sugar mixes tested, but it was unable to grow on D-fructose in the presence of a high and low concentration of L-rhamnose, suggesting that D-fructose uptake by RhtA was strongly inhibited by L-rhamnose (Fig 3A). D-fructose uptake by RhtA was also inhibited by L-arabinose, at a concentration of $66 \mathrm{mM}$, but not at a concentration of $6.6 \mathrm{mM}$. The results suggested that RhtA could have a higher affinity for L-rhamnose than for any of the other sugars tested. To determine the lower boundary for D-fructose uptake inhibition, the experiment was then repeated with lower L-rhamnose concentrations $(0.0006 \mathrm{mM}$ to $6.1 \mathrm{mM})$ in the 


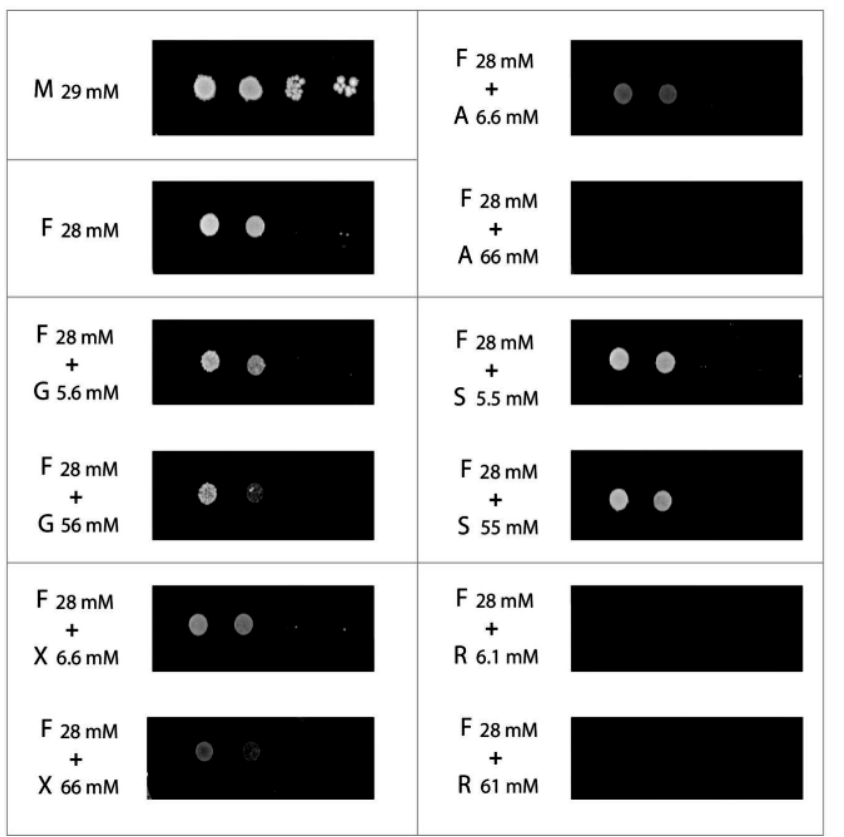

b)

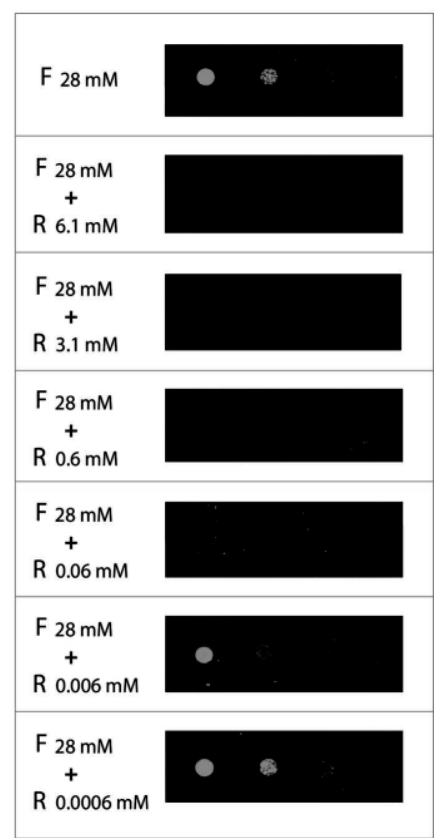

Figure 3 EBY.VW4000 rhaA+ growth inhibition assays. a) Growth of yeast strain EBY.VW4000 expressing the $r h t A$ gene on minimal medium with maltose (M; $29 \mathrm{mM})$, D-fructose (F; $28 \mathrm{mM})$, and D-fructose (F; $28 \mathrm{mM})$ supplemented with the potentially competing carbon sources: D-glucose (G; $56 \mathrm{mM}$ and $5.6 \mathrm{mM})$, Dxylose (X; $66 \mathrm{mM}$ and $6.6 \mathrm{mM}$ ), L-arabinose (A; $66 \mathrm{mM}$ and $6.6 \mathrm{mM})$, D-sorbitol (S; $55 \mathrm{mM}$ and $5.5 \mathrm{mM}$ ) or L-rhamnose (R; $61 \mathrm{mM}$ and $6.1 \mathrm{mM})$; b) growth of yeast strain EBY.VW4000 expressing the $r h t A$ gene on minimal medium with Dfructose $(\mathrm{F} ; 28 \mathrm{mM})$, and $\mathrm{D}$-fructose $(\mathrm{F} ; 28 \mathrm{mM})$ supplemented with a range of Lrhamnose concentrations $(0.0006 \mathrm{mM}$ to $6.1 \mathrm{mM})$

presence of D-fructose (28 mM) as carbon source (Fig 3B). As shown in Fig 3B, growth was inhibited by L-rhamnose concentrations as low as $0.06 \mathrm{mM}$. The fact that such a low concentration of L-rhamnose, around 500 times lower than of Dfructose, was able to inhibit growth, pointed to the deoxy sugar as a possible true substrate of the RhtA transporter. To prove that the strong growth inhibitory capacity of L-rhamnose was exclusively associated to the RhtA transporter, and not due to an unexpected toxicity of L-rhamnose on the yeast itself, a different strain (CEN.PK2-1C), isogenic to EBY.VW4000, harboring the p426HXT76His-rht $A$, was also grown in the same conditions. The CEN.PK2-1C strain expressing RhtA was able to grow in the presence of D-fructose $28 \mathrm{mM}+\mathrm{L}$ rhamnose $0.06 \mathrm{mM}$ (Fig 4A), thus indicating that the D-fructose uptake inhibition invoked by L-rhamnose was RhtA dependent, as inhibition of growth did not occur in the yeast strain with functional endogenous D-fructose transport 
systems. Finally, a competition plate assay was performed using two alternative deoxy sugars: L-fucose $(\mathrm{Fc} ; 6.1 \mathrm{mM})$ and 2-deoxy-D-ribose (Dr; $7.5 \mathrm{mM})$. The RhtA transformant showed normal growth, as can be observed in (Fig 4B). Taken together, these results suggested that $A$. niger RhtA could be a very specific transporter for L-rhamnose, unable to use other deoxy sugars as substrates.

a)

$\mathrm{M} 29 \mathrm{mM}$

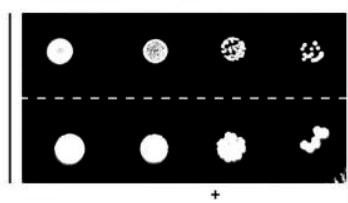

CEN.PK2-1C rhtA ${ }^{+}$

EBY.VW.4000 rhtA ${ }^{+}$

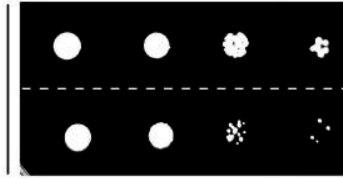

CEN.PK2-1C rhtA ${ }^{+}$

$\mathrm{F} 28 \mathrm{mM}$

$+$

$\mathrm{R} 0.06 \mathrm{mM}$

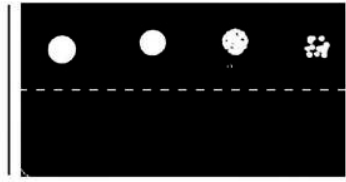

b)
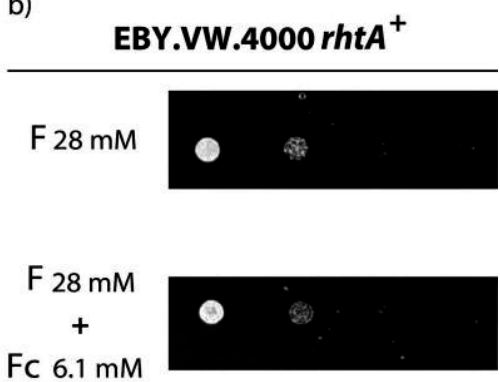

CEN.PK2-1C rhtA ${ }^{+}$

EBY.vW.4000 rhtA ${ }^{+}$

EBY.VW.4000 rhtA ${ }^{+}$

Fc $6.1 \mathrm{mM}$

$\mathrm{F} 28 \mathrm{mM}$

$+$

Dr $7.5 \mathrm{mM}$

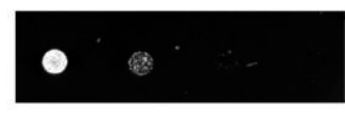

Figure 4 EBY.VW4000 rhaAt and CEN.PK2-1C rhaA+ growth inhibition assays. a) Growth of yeast strains CEN.PK2-1C and EBY.VW4000 expressing the $r h t A$ gene on minimal medium with maltose $(\mathrm{M} ; 29 \mathrm{mM}), \mathrm{D}$-fructose $(\mathrm{F} ; 28$ $\mathrm{mM})$ or D-fructose (F; $28 \mathrm{mM})$ supplemented with L-rhamnose (R; $0.06 \mathrm{mM}) ; \mathrm{b})$ Growth of EBY.VW4000 rhaA+ on minimal medium containing D-fructose (F; $28 \mathrm{mM})$ or D-fructose $(\mathrm{F} ; 28 \mathrm{mM})$ supplemented with L-fucose (Fc; $6.1 \mathrm{mM})$ or 2-deoxy-D-ribose (Dr; $6.1 \mathrm{mM})$ 


\section{Transcriptional analysis of the $\boldsymbol{A}$. niger sugar transporter coding gene $\boldsymbol{r h t} A$}

According to the RhtA protein abundance profile obtained by plasmalemma proteome analysis, the expression of the transporter seemed to be specifically induced by L-rhamnose. This fact, together with the additional findings described above, indicated that RhtA could be a Lrhamnose transporter. However, in yeast complementation experiments RhtA showed an ability to transport D-fructose, so in A. niger the transporter's biological role could be related to D-fructose uptake as well. Having this into account, new fermentations of the A. niger N400 wild type strain were performed, using the same set-up as described above, in order to study the transcriptional response of rhtA to D-sorbitol $100 \mathrm{mM}$ (reference), L-rhamnose $5 \mathrm{mM}$, and D-fructose $5 \mathrm{mM}$. Samples, obtained two hours after mycelium transfer, were processed, and RT-qPCR analysis was performed. As expected, the rhtA gene was strongly induced in the presence of L-rhamnose, while its expression levels in the presence of D-fructose were found to be similar to those observed in the reference condition (Fig 5). This result showed that L-rhamnose, but not D-fructose, acts as an inducer of the RhtA transporter at transcriptional level, suggesting that RhtA is not a natural Dfructose transporter. As mentioned above, the gene rhaB, coding for a

\section{rhtA}

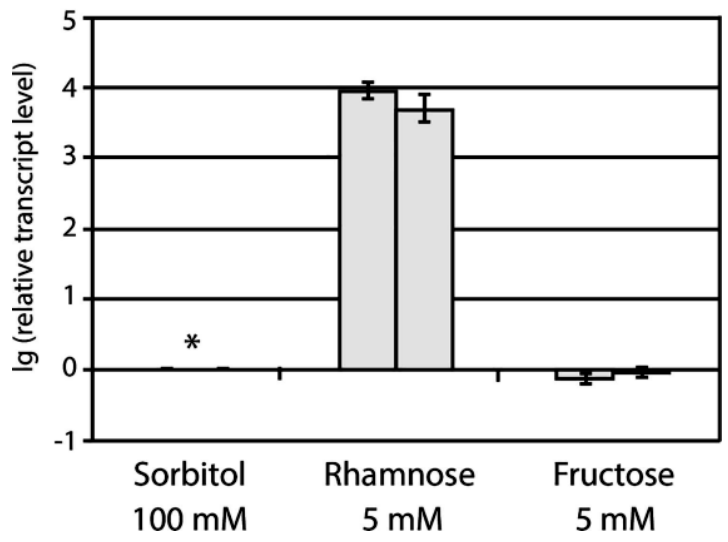

Figure 5. Transcriptional analysis of $r h t A$. Relative transcription levels were measured by RT-qPCR, in A. niger N400 sampled 2 hours after mycelium transfer to minimal medium with $100 \mathrm{mM}$ D-sorbitol (ref), $5 \mathrm{mM}$ L-rhamnose or $5 \mathrm{mM} \mathrm{D}$ fructose. Transcript levels are relative to reference sample (D-sorbitol $100 \mathrm{mM}$ ), indicated with an asterisk. Results are given as relative transcript ratios in log scale $(\lg (10))$. The values provided in the figures correspond to two biological replicates per culture condition. Error bars are means of three technical replicates 
hypothetical $\alpha$-L-rhamnosidase (JGI $A$. niger ATCC 1015 Protein ID 131668), was found to be located directly upstream of $r h t A$. The amino acid analysis of the hypothetical $\alpha$-L-rhamnosidase revealed the presence of a classical $\mathrm{N}$-terminal secretion signal peptide. Thus, it would be possible that both $r h a B$ and $r h t A$ gene products have a coordinated action, releasing and transporting L-rhamnose monomers respectively. This would imply that both proteins are coordinately expressed in the presence of the sugar. To obtain more insight into the transcriptional responses of rhtA and $r h a B$ to L-rhamnose, time course fermentations were done, in cultures containing either D-sorbitol $100 \mathrm{mM}$, Lrhamnose $1 \mathrm{mM}$ or L-rhamnose $5 \mathrm{mM}$ as sole carbon source. Sampling was performed every hour after mycelium transfer, until the L-rhamnose was depleted from the medium for two hours. The RT-qPCR results obtained showed a fast transcriptional response of $r h t A$ to the presence of L-rhamnose (Fig 6), while in the D-sorbitol reference condition rhtA expression remained at low level (S2 Fig). One hour after mycelium transfer the $r h t A$ expression level in the presence of an initial L-rhamnose concentration of $1 \mathrm{mM}$ was approximately 1000 fold higher than those observed in the reference condition (D-sorbitol $100 \mathrm{mM} ; \mathrm{t}=$ 1h). A maximum was observed two hours after transfer, with $r h t A$ transcriptional levels being 5000 fold higher than in the reference condition. The rhtA transcription profile for the first three hours was the same in the $5 \mathrm{mM} \mathrm{L}$ rhamnose fermentation (S2 Fig). The maximum $r h t A$ expression levels remained constant during the time course experiment until the deoxy sugar was completely consumed (Fig 6). After this point, rht $A$ mRNA levels decreased approximately 80 fold. Once the rhtA mRNA levels decreased, they remained constant at least 2 hours, being still around 50 times higher than its expression levels in the reference condition. High mRNA stability could be the reason why the rhtA expression levels kept being relatively high, even several hours after the sugar was completely consumed. The results displayed in Fig 6 show that rhaB has a similar expression profile to the one observed for $r h t A$ : its expression was activated in the presence of L-rhamnose and remained constant until the sugar was depleted from the medium (reference condition: D-sorbitol $100 \mathrm{mM} ; \mathrm{t}=1 \mathrm{~h}$ ). Therefore both genes seem to have a specific coordinated response to the presence of L-rhamnose in the environment. To further understand the regulatory mechanisms underlying the L-rhamnose uptake system encoded by the rht $A$ and $r h a B$ genes, the role of the regulators RhaR, involved in L-rhamnose release and 
catabolism [18], and CreA, mediating carbon catabolite repression in plant cell wall utilization systems [46], was studied.

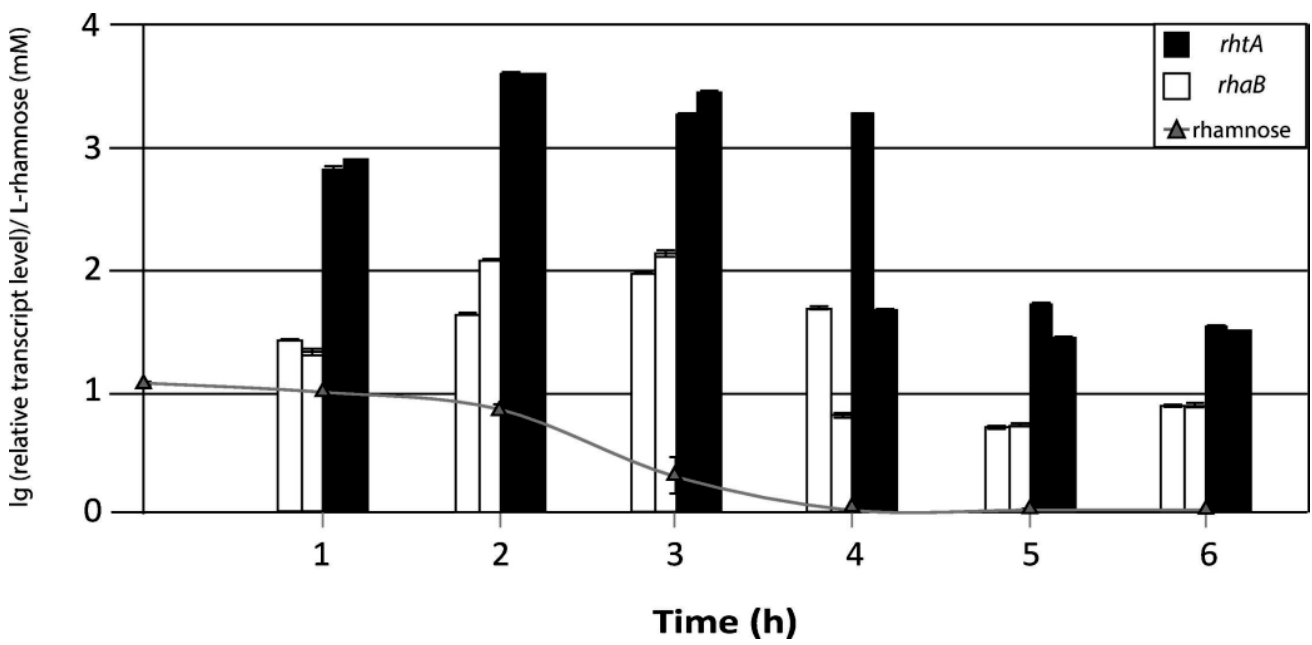

Figure 6 Time course transcriptional analysis of $r h t A$ and $r h a B$. Relative transcription levels, measured by RT-qPCR, of $r h t A$ (black bars) and $r h a B$ (white bars) during $A$. niger N400 fermentations in minimal medium with an initial concentration of L-rhamnose $1 \mathrm{mM}$. Concentration of L-rhamnose over time is represented by grey line with triangles (concentration at $t=4 \mathrm{~h}$ is equal to 0 ). Transcript levels of both genes always refer to the reference sample (D-sorbitol 100 $\mathrm{mM} ; \mathrm{t}=1 \mathrm{~h}$ ). Results are given as relative transcript ratios in logarithmic scale $(\lg (10))$. The values provided in the figures correspond to two biological replicates per culture condition. Error bars are means of three technical replicates 
rhtA

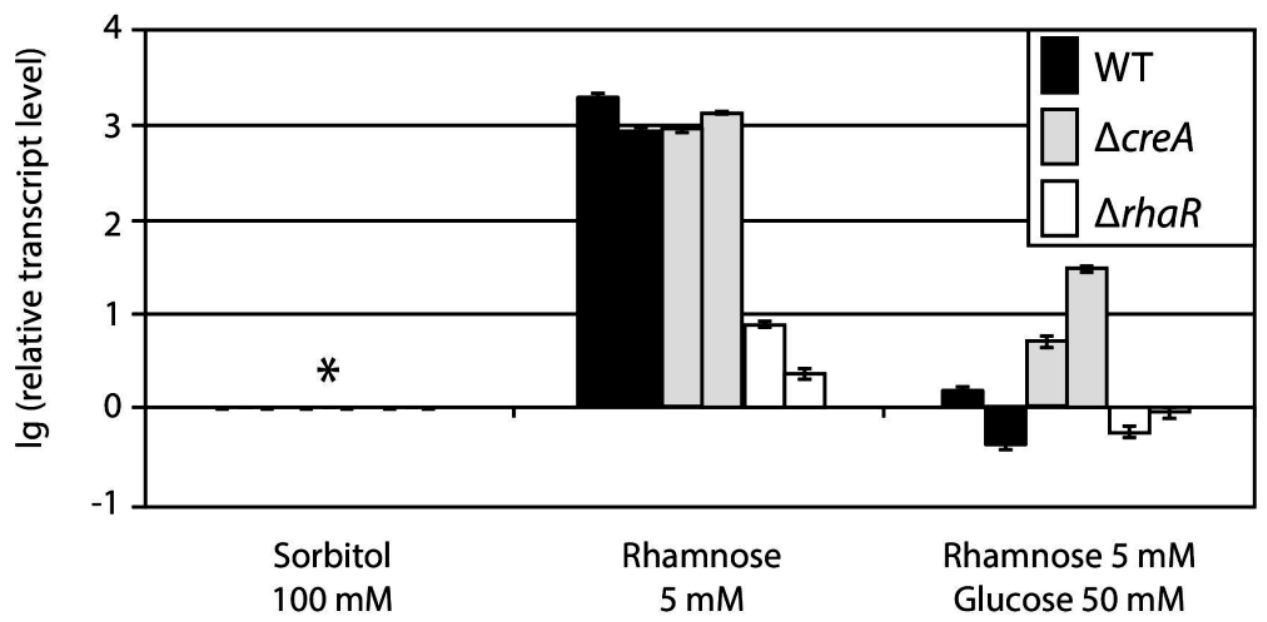

rhaB

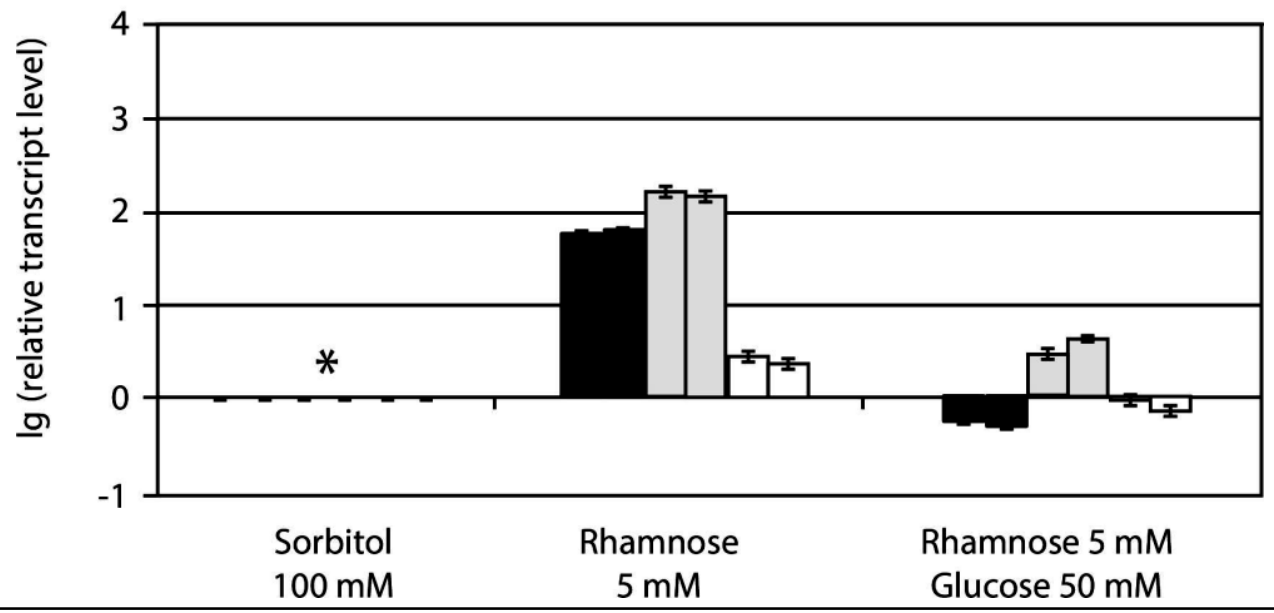

Figure 7 Role of the CreA and RhaR transcriptional regulators on the expression of $r h t A$ and $r h a B$. Strains N402 (WT; black bars), NW283 ( $\Delta c r e A$; grey bars), and JS014 ( $\Delta r h a R$; open bars) were used. Relative transcription levels were measured by RT-qPCR in samples obtained 2 hours after mycelium transfer to $5 \mathrm{mM} \mathrm{L}$ rhamnose or $5 \mathrm{mM}$ Lrhamnose plus $50 \mathrm{mM}$ D-glucose. Relative transcript levels of $r h t A$ and $r h a B$ were calculated using the pre-culture condition of each strain (Dsorbitol $100 \mathrm{mM} ; \mathrm{t}=18 \mathrm{~h}$ ), sampled prior to the mycelium transfer to inducing and inducing/ repressing conditions, as reference $(*)$. Results are given as relative transcript ratios in logarithmic scale $(\lg (10))$. The values provided in the figures correspond to two biological replicates per culture condition. Error bars are means of three technical replicates 
To do this, a transcriptional analysis of both genes in the strains N402 (WT), NW283 ( $\triangle c r e A)$ and JS14 ( $\triangle r h a R$ ) (see Materials and Methods section for construction details) was done. The wild type and the $\Delta c r e A$ and $\Delta r h a R$ strains were pre-cultured in MM with $100 \mathrm{mM} \mathrm{D}$-sorbitol for $18 \mathrm{~h}$, and transferred to MM with either $5 \mathrm{mM}$ L-rhamnose or $5 \mathrm{mM}$ L-rhamnose plus $50 \mathrm{mM}$ D-glucose. Samples were taken two hours after mycelium transfer, and were subsequently processed for RT-qPCR analysis. As previously observed, both rhtA and rhaB were induced by L-rhamnose. Their transcriptional levels in the presence of the deoxy sugar were similar in both the wild type (N402) and AcreA (NW283) strains (Fig 7). In the presence of L-rhamnose plus D-glucose, rht A and rhaB were heavily repressed in the wild type strain, and only partly derepressed in the $\triangle$ cre $A$ mutant. Regarding the regulatory mechanisms mediating the activation of $r h t A$ and $r h a B$, the expression of both genes in the presence of L-rhamnose was strongly reduced in the $\Delta r h a R$ strain, suggesting that the RhaR transcriptional activator is responsible for the induction of these genes.

\section{Determination of the RhtA ability to transport L-rhamnose}

To prove that RhtA can use L-rhamnose as substrate, a tritium labeled L- $\left[{ }^{3} \mathrm{H}(\mathrm{G})\right]-$ rhamnose uptake experiment was performed. In this experiment, the L-rhamnose uptake ability of the EBY.VW4000_RhtA strain was determined, using as a negative control the EBY.VW4000 strain expressing the A. niger specific Dxylose transporter XltB [47]. Additionally, to further investigate the RhtA transporter selectivity, D-[1-14 $\mathrm{C}]$-xylose and $\mathrm{D}-\left[{ }^{14} \mathrm{C}(\mathrm{U})\right]$-fructose were also tested as possible substrates. As shown in Fig 8, the RhtA strain showed a Lrhamnose uptake rate of $5.28 \times 10^{-3} \pm 0.87 \times 10^{-3} \mathrm{nmol} \mathrm{min}_{-1} \mathrm{mg} \mathrm{DW}_{-1}$, while the transport of the methylpentose by the control strain could not be detected. This

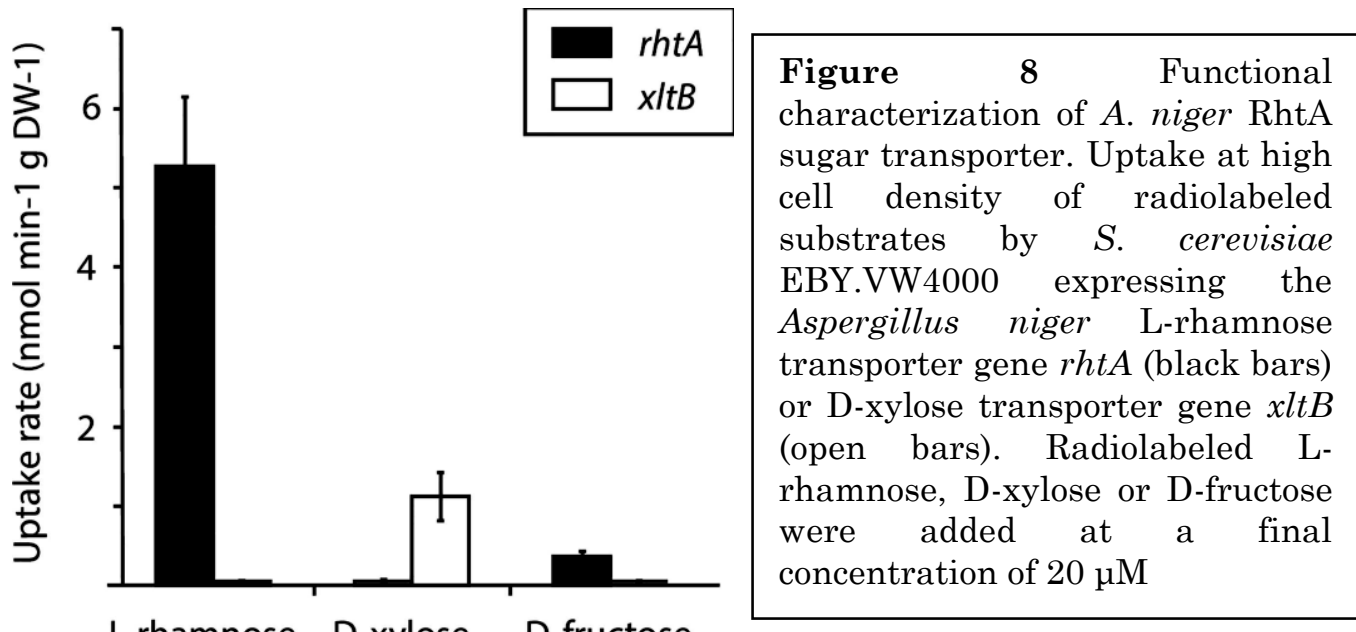


result, together with the previous findings reported in this study, confirmed that RhtA is a functional L-rhamnose transporter in A. niger. As expected, the control strain EBY.VW4000_XltB strain was able to transport D-xylose, with an uptake rate of $1.12 \times 10^{-3} \pm 0.31 \times 10^{-3} \mathrm{nmol} \mathrm{min}_{-1} \mathrm{mg} \mathrm{DW}_{-1}$, while the RhtA expressing strain could not. Finally, radiolabeled D-fructose uptake measurements confirmed the observations previously done in the functional complementation studies performed for XltB and RhtA: the EBY.VW4000 RhtA transformant could transport D-fructose with an uptake rate of $0.37 \times 10^{-3} \pm 0.06 \times 10^{-3} \mathrm{nmol} \mathrm{min}$ ${ }_{1} \mathrm{mg}$ DW, while D-fructose uptake by the XltB strain could not be detected. The L-rhamnose uptake rate determined for RhtA is significantly lower than that one reported for the E. coli L-rhamnose transporter [43], however, the experimental approach used by Tate et al., which performed overnight cultivations of different E. coli strains (WT and L-rhamnose negative strains) for that purpose, make a comparison difficult. The L-rhamnose uptake rate determined for RhtA was, however, comparable to the maximum sugar uptake rate determined for other fungal MFS transporters, like the D-xylose transporters GXS1 from Candida intermedia [48] or XylH from Debaryomyces hansenii [49].

\section{Deletion analysis of the $A$. niger $r h t A$ and $r h a B$ genes}

To further understand the biological role of the L-rhamnose transporter and the hypothetical $\alpha$-L-rhamnosidase in $A$. niger, deletion strains of rhtA (JS16) and rhaB (JS19) were constructed (see Materials and Methods section for construction details). In each case, two knockout strains were isolated, and their growth phenotype was studied by plating them on minimal media supplemented either with D-glucose $(1 \% \mathrm{w} / \mathrm{v})$, L-rhamnose $(1 \% \mathrm{w} / \mathrm{v})$ or rhamnogalacturonan I (RG1) $(1 \% \mathrm{w} / \mathrm{v})$ as sole carbon source. The N402 wild type strain and the regulator mutant $\Delta r h a R$ were used as controls. Growth in D-glucose was comparable, whereas clear differences could be observed in L-rhamnose plates (Fig 9). In the presence of the methylpentose, the $\triangle r h a R$ mutants did not grow (as was described by Gruben et al. (2014) [18]). The two $\Delta r h t A$ mutants were severely affected, showing less growth and sporulation, while the $\triangle r h a B$ transformants showed a normal growth. The growth reduction observed with the $\Delta r h t A$ mutants suggests a relevant role for RhtA in L-rhamnose uptake. The fact that the $\triangle r h t A$ mutants are still able to grow in the presence of L-rhamnose as sole carbon source indicates that $A$. niger must possess at least one other transporter capable of transporting the monosaccharide. All strains grew poorly in the presence of RG1, although slightly less growth could be observed in the $\triangle r h a R$ mutant. 


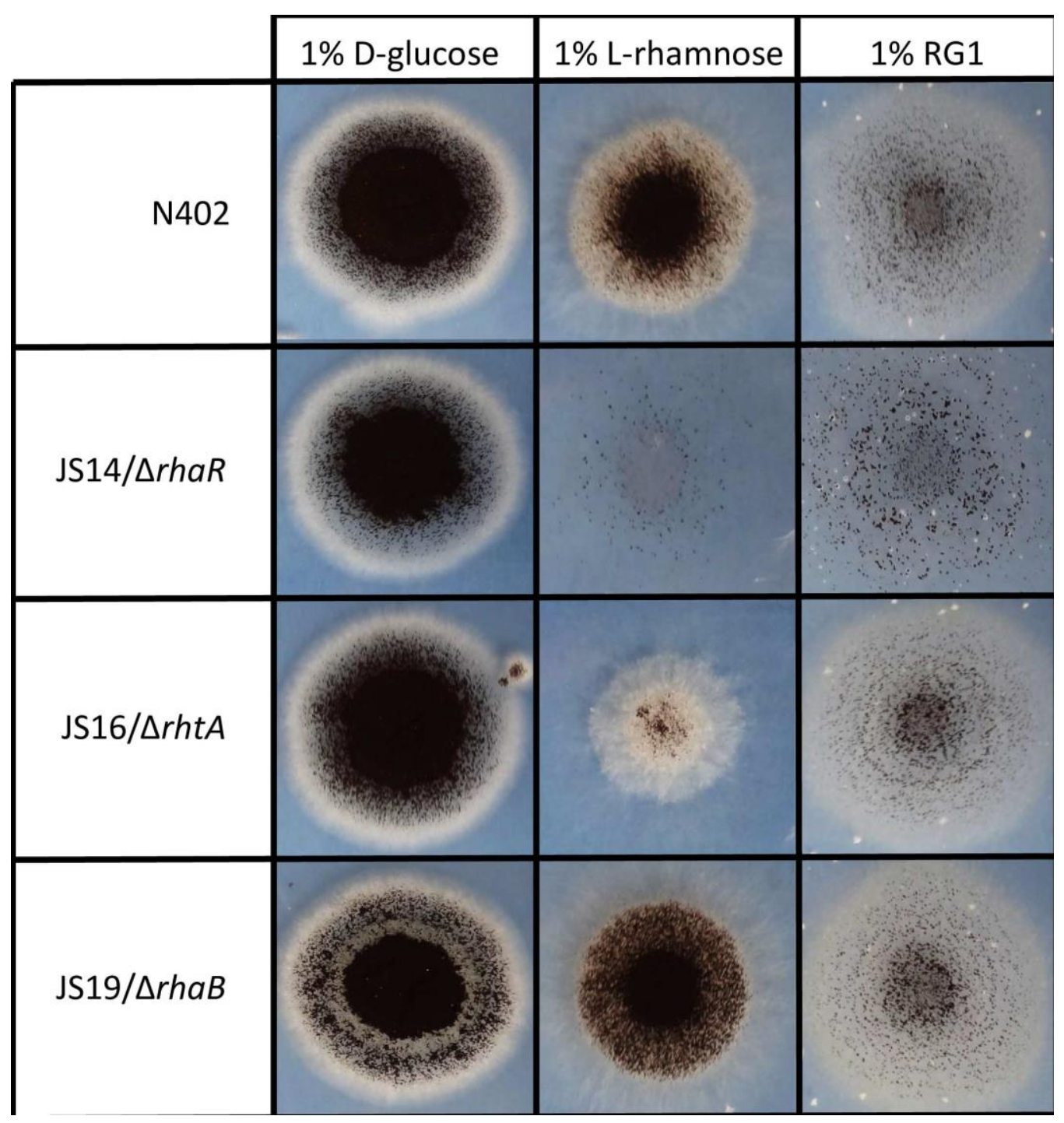

Figure 9 Phenotype analysis of $A$. niger strains N402 (WT), JS14 ( $\Delta r h a R)$, JS16 $(\Delta r h t A)$ and JS19 $(\Delta r h a B)$. A. niger strains were plated on minimal media supplemented either with D-glucose (1\%; w/v), L-rhamnose $(1 \%$; w/v) or rhamnogalacturonan I (RG1) (1\%; w/v) as sole carbon source, and cultured for 144 hours. Mutants with the same gene deleted showed the same growth pattern; the figure depicts only one representative knockout strain per gene 


\section{Discussion}

The prediction of substrate specificity of (sugar) transporters by using general classification systems is difficult, and traditional methods based on shared primary sequence similarity (e.g. the standard Blast algorithm) are in many cases not precise enough [47,50]. The use of profile hidden Markov models (HMM) to segregate sugar transporter proteins based on their substrate has been shown as an effective approach, however their precision largely depends on the availability of a consistent training set of biochemically characterized proteins with the function of interest $[36,47]$. In this study we aimed, for the first time, to identify a eukaryotic L-rhamnose transporter and consequently, no eukaryotic examples of L-rhamnose transporters were available in the literature. Bacterial examples of L-rhamnose transporters from E. coli and Salmonella typhimurium identified in the early 1990s [43] belong to the TC 2.A.7.6 transporters family, which has no similarity with MFS transporters, and have not been associated to the transport of sugars in eukaryotic organisms. To identify putative transporters specific for L-rhamnose we have taken advantage of the fact that $A$. niger possesses complex regulatory circuits that control tightly the expression of protein sets, including extracellular enzymes, transporter proteins and metabolic enzymes, specific for the utilization of different sugars [7]. Therefore, the analyzes of global transcriptomic and proteomic responses of the fungus to a variety of specific culture conditions can be useful approaches to get insights in the specific structural elements, including specific sugar transporters, required for the utilization of specific carbon sources. The activation of structural genes involved in L-rhamnose utilization by A. niger and other fungi have been shown to require the presence of an inducing carbon source which can be either L-rhamnose or pectic polysaccharides [16,18,51]. Assuming that the above also holds for Lrhamnose specific transporters, RhtA was selected by applying stringent differential protein expression criteria. Heterologous expression of candidate transporters in the $S$. cerevisiae monosaccharide transporter null strain EBY.VW4000 has been a very effective tool to study the function of single monosaccharide transporters in isolation. Although this strain naturally only utilizes hexoses like D-glucose and D-fructose, it has been amended to metabolize D-xylose or L-arabinose [52-54]. Accordingly, the strain can be used to screen for L-arabinose and D-xylose transporters. In a similar manner, a microbial L-rhamnose utilization pathway transferred to the EBY.VW4000 strain would allow screening for L-rhamnose transporters. By using an alternative approach, we managed to get important insights in the possible role of $A$. niger RhtA as a functional L-rhamnose transporter. First, we showed that RhtA is expressed as a functional transporter able to transport D-fructose. Subsequently, 
we determined that L-rhamnose had an extraordinary ability to specifically inhibit growth of the EBY.VW4000_RhtA strain, even at micromolar levels. This fact, plus additional insights observed at genomic, transcriptomic and proteomic level gave strong indications that RhtA acts as a specific transporter for Lrhamnose, but did not provide direct evidence. To assay transport capacity and quantify uptake kinetics, radiolabeled sugar uptake experiments can be performed with the mentioned yeast strain [33, 34, 36, 55-57]. This approach was followed in the present study as a final step to prove the ability of RhtA to transport L-rhamnose. Besides the ability of RhtA to transport L-rhamnose, we have obtained important insights into the biological role of the transporter. The tandem localization of rhtA with the $\alpha$-L-rhamnosidase coding gene rhaB, and their coordinated transcriptional response to the presence of L-rhamnose, indicated that both proteins could have a joint action in releasing and transporting the sugar. We also investigated if, besides rhaB, additional hypothetical $\alpha$-Lrhamnosidase genes (1180185/An08g09140, 1160525/An18g04800, 1132057/An01g06620, 1180185/ An15g04530, 1126821/An04g09070, 1134376/An07g00240, and 1165677/An10g00290; Protein accession numbers in ATCC 1015 and CBS 513.88 genomes) were co-localized with sugar transporters and noticed that the co-expressed $r h a B-r h t A$ tandem was a unique case in $A$. niger. The transcriptional profile of rhtA and $r h a B$ genes also suggests a coordinated role for the utilization of the deoxy sugar. In the presence of an initial L-rhamnose concentration of $1 \mathrm{mM}$ their expression was strongly induced while the sugar was being consumed. Concentrations even lower than $1 \mathrm{mM}$ might therefore also induce this system, as it occurs in the case of the D-xylose utilization system in $A$. niger, where a concentration of $0.1 \mathrm{mM}$ D-xylose already exerts a strong activation of structural genes like $x \ln B$ and $x \ln D$ [58]. Regarding the expression regulation of the transporter and the $\alpha$-L-rhamnosidase genes mediated by RhaR, we also analyzed the microarray expression data of $A$. niger WT and a $\triangle r h a R$ strain grown on L-rhamnose (accession number GSE51023) recently deposited by Gruben et al at GEO [18], observing that in this independent study using a different setup rhtA (An12g05710) and rhaB (An12g05700) were not induced in a RhaR knockout strain. Therefore, both microarray (Gruben et al) and RT-qPCR (this study) data analysis of two independent studies indicated that RhaR is responsible for rhtA and rhaB transcriptional activation. However, although the effect produced by RhaR in the regulation of these genes is very clear, as can be observed in Fig 7 their induction was not fully abolished in the $\Delta r h a R$ strain. This might indicate the influence of another transcriptional regulator that responds to the presence of L-rhamnose. Previously, a second transcriptional factor (FST14) was suggested to be involved 
in the regulation of the $P$. stipitis pectinolytic network [16], and this could also be the case in $A$. niger, as recently suggested by Gruben et al [18]. In the presence of L-rhamnose plus D-glucose, rhtA and $r h a B$ were heavily repressed in the wild type strain, and only partly derepressed in the $\triangle$ cre $A$ mutant. While this result suggests a role of CreA in controlling the expression of rhtA and rhaB, as reported before for other sugar utilization systems [11, 46, 59, 60], a different glucose-repression mechanism seems to have a major role in the transcriptional regulation of these genes. The existence of CreA-independent glucose repression mechanisms controlling the regulation of $\alpha$-L-rhamnosidase genes [13], and the phenylacetic acid uptake system [61], has been previously reported in A. nidulans. The isolation of $r h t A$ and $r h a B$ deletion strains allowed us to investigate the relevance of both proteins in A. niger. On one hand, the absence of RhtA produced a growth and sporulation defect in the $\Delta r h t A$ mutants on L-rhamnose containing media, underpinning the relevant biological role of RhtA for transport of L-rhamnose. Similar phenotype analyzes performed in filamentous fungi, where different sugar transporter mutant strains were studied, produced disparate results. In some cases, an altered growth phenotype could not be detected in plate assays containing the transporter's specific substrate [30, 55, 62, 63], which is probably due to overlapping substrate specificities. In this regard, the most notorious case corresponds to $S$. cerevisiae, where many genes had to be disrupted before its ability to transport D-glucose was abolished [29]. However, as we observed in the present study, the absence of certain transporters in filamentous fungi has also been shown to be accompanied with clear growth defects [64-66]. On the other hand, the absence of $r h a B$ did not affect negatively the mutant growth in the presence of RG1. This result is not surprising, bearing in mind that $\alpha$-L-rhamnosidase genes appear to be quite redundant in the $A$. niger genome. RhtA is, according to our knowledge, the first functionally validated eukaryotic transporter containing a FucP domain structure. It is also the first eukaryotic L-rhamnose transporter functionally validated to date, therefore this study provides major insights about the utilization of this monosaccharide by fungi. The identification of RhtA will also have an impact in the design of new microbial strains using L-rhamnose-rich biomass as feedstocks, like pectic polysaccharides from plants [67], or ulvan from green seaweeds [23,68], for the production of fuels and chemicals. 


\section{Materials and Methods}

\section{Strains and growth conditions}

The Aspergillus niger strains used in this study were N400 (NRRL3, ATCC9029, CBS120.49), N402 (cspA1) [69], NW283 (fwnAl; cspA1; lysA7 pyrA6; creAd4) [59], MA169.4 (cspA1, kusA:: DR-amdS-DR, pyrG-) [70], and its derivatives JS14, ( $\triangle r h a R)$, JS16 ( $\Delta r h t A)$ and JS19 ( $\triangle r h a B)$ constructed in this study. A. niger spores were generated on complete medium (CM) plates. Mycelial biomass for transfer experiments was produced in 18 hour pre-cultures after the inoculation of $10_{6}$ spores $\mathrm{ml}_{-1}$ in culture medium containing: $6 \mathrm{~g} \mathrm{~L}_{-1}(\mathrm{w} / \mathrm{v}) \mathrm{NaNO}_{3}, 1.5 \mathrm{~g} \mathrm{~L}-1$ (w/v) $\mathrm{KH}_{2} \mathrm{PO}_{4}, 0.5 \mathrm{~g} \mathrm{~L}_{-1}(\mathrm{w} / \mathrm{v}) \mathrm{KCl}, 0.5 \mathrm{~g} \mathrm{~L}-1$ (w/v) $\mathrm{MgSO}_{4} \mathrm{H}_{2} \mathrm{O}$ and Vishniac salts $[71,72]$, with $5 \mathrm{~g} \mathrm{~L}_{-1}(\mathrm{w} / \mathrm{v})$ yeast extract, D-sorbitol $100 \mathrm{mM}$, and the appropriate supplements to complement auxotrophic mutations (initial $\mathrm{pH}$ 6.0). For the plasma membrane proteomics analysis, equal amounts of water-rinsed mycelium of the wild type strain A. niger N400 were transferred to 1-liter benchtop fermenters (Sartorius) with $750 \mathrm{~mL}$ of minimal medium (MM) containing: $6 \mathrm{~g}$ $\mathrm{L}_{-1}(\mathrm{w} / \mathrm{v}) \mathrm{NaNO}_{3}, 1.5 \mathrm{~g} \mathrm{~L}-1$ (w/v) $\mathrm{KH}_{2} \mathrm{PO}_{4}, 0.5 \mathrm{~g} \mathrm{~L}-1$ (w/v) KCl, $0.5 \mathrm{~g} \mathrm{~L}-1$ (w/v) $\mathrm{MgSO}_{4} \mathrm{H}_{2} \mathrm{O}$, Vishniac salts, and one of the following carbon sources: L-arabinose $5 \mathrm{mM}$, D-mannose $5 \mathrm{mM}$, D-sorbitol $100 \mathrm{mM}$ plus D-xylose $0.1 \mathrm{mM}$, and Lrhamnose $5 \mathrm{mM}$. Two biological replicates per condition were studied. Fermenters were kept at $30^{\circ} \mathrm{C}$, stirred at $1000 \mathrm{rpm}$ and aerated with filtered air (0.6 L min-1), keeping oxygen levels over $60 \%$. The initial $\mathrm{pH}$, set at 4.0 , was allowed to drop until $\mathrm{pH} 3.5$ and kept constant afterwards by sodium hydroxide addition. These culture conditions were also used in the study of rhtA transcriptional response to D-sorbitol $100 \mathrm{mM}$, L-rhamnose $5 \mathrm{mM}$, and Dfructose $5 \mathrm{mM}$; and also in the time course fermentations in cultures containing D-sorbitol $100 \mathrm{mM}$, L-rhamnose $1 \mathrm{mM}$, or L-rhamnose $5 \mathrm{mM}$. For the analysis of the regulatory mechanisms controlling $r h t A$ and $r h a B$, the A. niger mycelial biomass of the strains N402, NW283 ( $\triangle c r e A)$ and JS14 ( $\triangle r h a R$ ) was produced in 18 hour pre-cultures (2 Erlenmeyer flasks per strain) after the inoculation of $10_{6}$ spores ml-1 in culture medium containing: $6 \mathrm{~g} \mathrm{~L}_{-1}(\mathrm{w} / \mathrm{v}) \mathrm{NaNO}_{3}, 1.5 \mathrm{~g} \mathrm{~L}-1(\mathrm{w} / \mathrm{v})$ $\mathrm{KH}_{2} \mathrm{PO}_{4}, 0.5 \mathrm{~g} \mathrm{~L}_{-1}(\mathrm{w} / \mathrm{v}) \mathrm{KCl}, 0.5 \mathrm{~g} \mathrm{~L}-1$ (w/v) $\mathrm{MgSO}_{4} \mathrm{H}_{2} \mathrm{O}$, Vishniac salts, $1 \mathrm{~g} \mathrm{~L}-1$ $(\mathrm{w} / \mathrm{v})$ yeast extract, $100 \mathrm{mM} \mathrm{D}$-sorbitol, and the appropriate supplements to complement auxotrophic mutations (initial $\mathrm{pH}$ 6.0). Equal amounts of mycelium from each strain were then transferred to $100 \mathrm{~mL}$ Erlenmeyer flasks with the same medium composition, containing $5 \mathrm{mM}$ L-rhamnose or $5 \mathrm{mM}$ L-rhamnose plus $50 \mathrm{mM}$ D-glucose as carbon sources. Cultures were performed in an orbital shaker for two hours at $30^{\circ} \mathrm{C}$ and $225 \mathrm{rpm}$. The phenotype analysis of the different $A$. niger mutant strains obtained in this study was done in agar plates, 
containing MM plus the appropriate supplements and carbon sources. The $S$. cerevisiae strain EBY.VW4000 (CEN.PK2-1C hxt134::loxP; hxt15::AloxP;

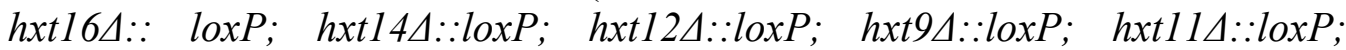

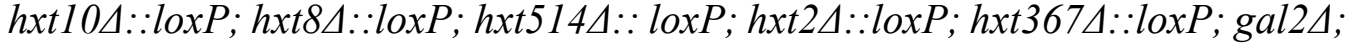

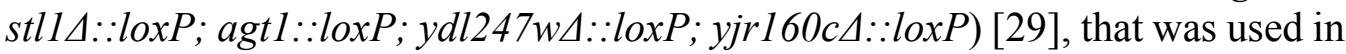
this study for the functional validation of the $r h t A$ gene, was grown at $30^{\circ} \mathrm{C}$ and maintained in solid complete medium containing $10 \mathrm{~g} \mathrm{~L}_{-1}(w / v)$ of yeast extract, $20 \mathrm{~g} \mathrm{~L}_{-1}(w / v)$ of peptone and $20 \mathrm{~g} \mathrm{~L}_{-1}(w / v)$ of maltose. The EBY.VW4000 derived strains obtained in the present study where grown in liquid minimal medium (MM) containing $6.7 \mathrm{~g} \mathrm{~L}_{-1}(w / v)$ of yeast nitrogen base with ammonium sulphate (w/o amino acids) (Difco), supplemented with leucine (30 $\left.\mathrm{mg} \mathrm{L}_{-1} ; \mathrm{w} / \mathrm{v}\right)$, tryptophan $\left(20 \mathrm{mg} \mathrm{L}_{-1} ; w / v\right)$ and histidine $\left(20 \mathrm{mg} \mathrm{L}_{-1} ; \mathrm{w} / \mathrm{v}\right)$, and using $20 \mathrm{~g} \mathrm{~L}_{-1}$ $(w / v)$ of maltose as carbon source. The $S$. cerevisiae wild type strain CEN.PK21C (MATa; his3A1; leu2-3_112; ura3-52; trp1-289; MAL2-8c; SUC2) strain was used as a control strain. For the yeast spot assays, the different $S$. cerevisiae strains were grown overnight as mentioned above and harvested in exponential phase by centrifugation. Cells were then diluted to the following optical densities at $600 \mathrm{~nm}$ (OD600): 1, 0.1, 0.01 and 0.001 , and subsequently $5 \mu 1$ droplets were spotted on $1.5 \%$ agar plates containing MMplus the appropriate supplements and different carbon sources. For radiolabelled sugars uptake experiments S. cerevisiae strains were cultured in baffled flasks with MMsupplemented with methionine and arginine for enhanced growth, and histidine, leucine and tryptophan to complement auxotrophic mutations. Incubations were done in an orbital shaker at $30^{\circ} \mathrm{C}$ and $225 \mathrm{rpm}$.

\section{A. niger membrane associate proteome purification, quality control analysis, sample preparation for LC-MS/MS and proteomics data analysis}

The A. niger membrane proteome preparation and purification was performed as described [36]. A. niger mycelium samples ( $2 \pm 3 \mathrm{~g}$, press-dried), washed and resuspended in $20 \mathrm{mM}$ HEPES buffer ( $\mathrm{pH} 7.6$ ) containing $150 \mathrm{mM} \mathrm{NaCl}$ and protease inhibitor cocktail for yeast \& fungi (Sigma-Aldrich), were mechanically disrupted using a French press (8000 psi). Three differential centrifugation steps, at low $(500 \mathrm{~g})$, medium $(5,000 \mathrm{~g})$ and high speed $(\sim 85,000 \mathrm{~g})$, were performed to pellet light organelles (P3). P3 pellets were resuspended using a Dounce homogenizer in $1 \mathrm{~mL}$ of $20 \mathrm{mM}$ HEPES buffer ( $\mathrm{pH} 7.6$ ), containing $250 \mathrm{mM}$ sucrose. The P3 suspensions were subsequently overlaid in a discontinuous sucrose density gradient, prepared by layering successive solutions, with decreasing sucrose concentrations $(6 \times 1 \mathrm{~mL} ; 1.20 \mathrm{M}$ to $0.70 \mathrm{M})$, upon one another. Sucrose density gradients were centrifuged $(\sim 100,000 \mathrm{~g} \pm 60 \mathrm{~min})$ to 
isolate different membrane-associated fractions from $\mathrm{P} 3$ pellet. Five fractions were obtained (P3A, P3B, P3C, P3D and P3E). The plasma membrane (PM) marker vanadate-sensitive $\mathrm{H}+$ ATPase and the mitochondrial membrane cytochrome c oxidase activities were then measured in the initial cell free extract, the $\mathrm{P} 3$ pellet and the P3A to $\mathrm{P} 3 \mathrm{E}$ fractions derived from it. Compared to the cell free extract, the P3 pellet was 2.4 to 3.2 times enriched in plasma membranes. No further enhanced PM enrichment was found in the P3A to P3E fractions, therefore the $\mathrm{P} 3$ pellets were considered to be more optimal for the analysis of plasmalemma proteins, that were further processed and subjected to shotgun proteomics analysis. Cytochrome c oxidase activity measurements were performed using the ${ }^{\mathrm{a}} \mathrm{Cytochrome} \mathrm{c}$ Oxidase Assay $\mathrm{Kit}^{\mathrm{o}}$ from Sigma-Aldrich (CYTOCOX1), following the user manual. The vanadate-sensitive H + ATPase enzyme assay was performed as described previously [73]. The protocol used in order to prepare membrane proteins for LC-MS/MS analysis has been described in detail previously [36]. LC-MS/MS analyzes were done at Radboud Proteomics Centre as described previously [74]. The obtained raw mass spectrometry proteomics data was deposited to the ProteomeXchange Consortium via the PRIDE partner repository with the dataset identifier PXD004909. The analysis of the LC-MS/MS spectra obtained from the proteomics experiment, were identified and quantified using the MaxQuant software [75], as described [36].

\section{Construction of $S$. cerevisiae EBY.VW4000 strain expressing $A$. niger rht $A$ gene}

The chemically synthesized rhtA cDNA coding sequence (A. niger ATCC 1015 JGI prot. ID: 1119135) was PCR amplified from plasmid using primers HE_rhtA_FW and HE_rhtA_RV (S2 Table), using Phusion polymerase (ThermoFisher Scientific) following the manufacturers protocol. The fragment was then digested with SpeI and XhoI and cloned into the S. cerevisiae expression vector p426HXT7-6His, linearized with the same restriction enzymes, under the control of the constitutive promoter HXT7p and the terminator CYC1t. Transformation of $S$. cerevisiae EBY.WV4000 was performed as described previously [76].

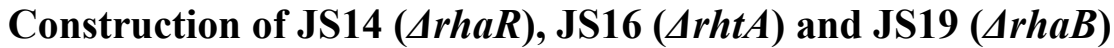

Using the split-marker approach, the previously identified L-rhamnose regulator gene rhaR (JGI ATCC 1015 Prot ID 1116273) [18], the rhamnose transporter gene rhtA (JGI ATCC 1015 Prot ID 1119135), or the putative rhamnosidase gene rhaB (JGI ATCC 1015 Prot ID 131668) were deleted from the genome of the MA169.4 strain (isogenic of N402), which is defective in the Non-Homologous 
End-Joining (NHEJ) pathway through a transiently silenced kusA gene [70,77]. A schematic representation of the four experimental steps required can be found in S3 Fig, PCR results that confirm the correct deletion of the genes from the genome can be found in S4 Fig and primers used are listed in S2 Table. As an example we describe the construction of JS14 ( $\Delta r h a R$ ) knockout strains, the other strains were constructed in the same manner with the corresponding primers. First, homologous regions were amplified from A. niger N402 genomic DNA using primers $\mathrm{KO} \_r h a R 55^{\prime} \mathrm{FW}$ with $\mathrm{KO}$ rhaR5'_RV and $\mathrm{KO}$ rhaR3'_FW with $\mathrm{KO}$ rhaR3' RV, and the marker gene was amplified from pAO4-13 using primers $\mathrm{KO} \_$pyrG_FW and $\mathrm{KO} \_p y r G_{-} \mathrm{RV}$. Second, these three fragments were used as template to create marker-flank fusion fragments using primers $\mathrm{KO}$ rhaR5'_ $\mathrm{FW}$ with $\mathrm{KO} \_$pyrG2_RV and $\mathrm{KO}$ pyrG2_FW with KO_rhaR3'_RV. Third, the resulting fragments were used to transform MA169.4 as previously described [78]. Single $A$. niger transformant colonies were purified and the transiently silenced $k u s A$ gene was restored on MM plates containing fluoroacetic acid (FAA). Finally, correct marker localization in the strain JS14 ( $\triangle r h a R$ ) was checked by PCR using genomic DNA as template, and the primer pairs CH_locusrhaR_FW with $\mathrm{KO} \_$pyrG2_RV and $\mathrm{KO}$ pyrG2_FW with CH_locusrhaR_RV. Deletion of the rhaR gene was confirmed by PCR using the primers $\mathrm{CH}$ rhaR_FW and $\mathrm{CH} \_r h a R \_\mathrm{RV}$.

\section{RNA extraction and transcriptional analysis of $r h t A$ and $r h a B$ genes}

Mycelium samples were disrupted with glass beads in a Fastprep-24 instrument, and RNA was isolated using a Maxwell 16 instrument using the Maxwell 16 LEV simplyRNA kit (Promega). Reverse transcription and qPCR analysis were performed following the protocols and instruments described in Mach-Aigner et al., 2012 [79]. In short, after treatment with DNase I, cDNA was synthesized from $0.45 \mu \mathrm{g}$ RNA using the RevertAid H Minus First Strand cDNA synthesis kit (Thermo Fisher). All reactions were performed according to the manufacturer's instructions. All quantitative PCRs (qPCRs) were performed in triplicate in a Rotor-Gene 3000 cycler (Qiagen). The amplification mixture (final volume, $15 \mu \mathrm{l}$ ) contained $7.5 \mu \mathrm{l}$ of $2 \mathrm{x}$ ABsolute QPCR SYBR Green mix, 100 $\mathrm{nM}$ forward and reverse primers and $2.5 \mu \mathrm{cDNA}$ (diluted 1:100). The primers used for qPCR analysis were designed using the software Quant- Prime [80], and are listed in S2 Table. Each run included a no-template control and a noamplification control $(0.015 \%$ SDS added to the reaction mixture). The cycling conditions comprised a $15 \mathrm{~min}$ initial polymerase activation at $95^{\circ} \mathrm{C}$, followed by 40 cycles of $95^{\circ} \mathrm{C}$ for $15 \mathrm{~s}, 59^{\circ} \mathrm{C}$ for $15 \mathrm{~s}$, and $72^{\circ} \mathrm{C}$ for $15 \mathrm{~s}$. The previously described histone-like gene hist transcript (A. niger ATCC 1015 gene ID 207921) 
and the Golgi Transporter gene (A. niger CBS 513.88 An02g04120) were used as reference for normalization of the expression data [58,79]. Dissociation (or melting) curve analysis was performed on each qPCR reaction to confirm that the primer pairs used produced a single amplification product. Results are given as relative transcript ratios in logarithmic scale $(\lg (10))$. The values provided in the figures correspond to two biological replicates per strain and culture condition.

\section{Sugars analysis}

Sugars present in the $A$. niger culture supernatants were measured through highpressure liquid chromatography (HPLC) analysis. A Thermo Accela equipped with a Shodex KC-811 column, coupled to a refractive index detector (Spectrasystem RI-150, sample frequency $5.00032 \mathrm{~Hz}$ ) and a UV-VIS detector (Spectrasystem UV1000, $\lambda: 210 \mathrm{~nm}$ ), was used. Separations were performed by isocratic elution with $0.01 \mathrm{~N} \mathrm{H}_{2} \mathrm{SO}_{4}$, at a flow rate of $0.8 \mathrm{~mL}$ min-1. Crotonate (6 $\mathrm{mM}$ ) was used as an internal standard.

\section{Radiolabeled sugar uptake determinations}

Sugar uptake assays were performed as described previously, with minor adjustments [81]. Liquid cultures using MM, supplemented with methionine (20 $\mathrm{mg} \mathrm{L-1} ; \mathrm{w} / \mathrm{v})$, arginine (20 mg L-1; w/v), leucine (30 mg L-1; w/v), tryptophan (20 $\mathrm{mg} \mathrm{L}-1 ; \mathrm{w} / \mathrm{v})$ and histidine $(20 \mathrm{mg} \mathrm{L}-1 ; \mathrm{w} / \mathrm{v})$, with $1 \%(\mathrm{w} / \mathrm{v})$ maltose as carbon source, were inoculated with strains EBY.VW4000 XltB (control), and EBY.VW4000_RhtA, and incubated for 5 days. Cells were then harvested by centrifugation (4000 g, $10 \mathrm{~min}$ ), washed with $50 \mathrm{~mL}$ ice-cold ultrapure water, and washed again with ice-cold PBS ( $\mathrm{pH}$ 6.5). Cells were then resuspended in PBS (pH 6.5), divided in $40 \mu \mathrm{L}$ aliquots, and kept on ice. Aliquots were incubated for $5 \mathrm{~min}$ at $30^{\circ} \mathrm{C}$ before the uptake assay was started. To start the assay, $10 \mu \mathrm{l}$ of a $100 \mu \mathrm{ML}-\left[{ }^{3} \mathrm{H}(\mathrm{G})\right]$-rhamnose, D- $\left[{ }^{14} \mathrm{C}(\mathrm{U})\right]$-fructose or D-[1- $\left.{ }^{14} \mathrm{C}\right]$-xylose solution (Campro Scientific, Veenendaal) was added. After exactly 20 seconds the reaction was quenched by the addition of $1 \mathrm{~mL}$ of ice-cold wash buffer (PBS, $\mathrm{pH}$ 6.5 , with $500 \mathrm{mM}$ of the corresponding non-labeled substrate solution), followed by a vacuum filtration step $(0.45 \mu \mathrm{m}$ HV filters, 1225 sampling manifold, Millipore), and two subsequent washing steps with $5 \mathrm{~mL}$ of ice-cold washing buffer. After drying for $5 \mathrm{~min}$ in the vacuum manifold, the filters were transferred to scintillation vials with $5 \mathrm{~mL}$ scintillation liquid (Ultima Gold, Perkin Elmer), and activity was counted (Packard Tricarb 1600TR). All reactions were performed in triplicate. Negative control reactions, where quenching was done 
before substrate addition and without incubation, were performed for each reaction.

\section{Bioinformatic analysis}

DNA and protein sequences were obtained from the JGI A. niger ATCC 1015 genome database [37]. Retrieved sequences were subsequently used in additional searches using the BLAST tools at the NCBI database. Protein transmembraneaware multiple alignments were done using a PRALINE, incorporating TMHMM2.0 transmembrane helix prediction tool [82,83]. SignalP4.1 was used to detect the signal peptide for secretion in the RhaB protein [84]. 


\section{References}

1. The Fungal Community: Its Organization and Role in the Ecosystem, Second Edition [Internet]. CRC Press; 1992. Available: https://books.google.com/books?id=ikJfKaz0lEEC\&pgis=1

2. Carlile MJ, Watkinson SC, Gooday GW. The Fungi [Internet]. The Fungi. Elsevier; 2001.

3. Andersen MR, Salazar MP, Schaap PJ, van de Vondervoort PJI, Culley D, Thykaer J, et al. Comparative genomics of citric-acid-producing Aspergillus niger ATCC 1015 versus enzyme-producing CBS 513.88. Genome Res. 2011; 21: 885-97. doi: 10.1101/gr.112169.110 PMID: 21543515

4. de Vries RP, Visser J. Aspergillus enzymes involved in degradation of plant cell wall polysaccharides. Microbiol Mol Biol Rev. 2001; 65: 497-522, table of contents. doi: 10.1128/MMBR.65.4.497-522.2001 PMID: 11729262

5. van.Peij NME, Gielkens MMC, de Vries RP, Visser J, de Graaff LH. The Transcriptional Activator XlnR Regulates Both Xylanolytic and Endoglucanase Gene Expression in Aspergillus niger. Appl Environ Microbiol. 1998; 64: 36153619. Available: http://aem.asm.org/content/64/10/3615.short PMID: 9758775

6. Battaglia E, Hansen SF, Leendertse A, Madrid S, Mulder H, Nikolaev I, et al. Regulation of pentose utilization by AraR, but not XlnR, differs in Aspergillus nidulans and Aspergillus niger. Appl Microbiol Biotechnol. 2011; 91: 387-97. doi: 10.1007/s00253-011-3242-2 PMID: 21484208

7. De Souza WR, Maitan-Alfenas GP, de Gouvêa PF, Brown NA, Savoldi M, Battaglia E, et al. The influence of Aspergillus niger transcription factors AraR and $\mathrm{X} \operatorname{lnR}$ in the gene expression during growth in dxylose, 1-arabinose and steam-exploded sugarcane bagasse. Fungal Genet Biol. Elsevier Inc.; 2013; 60: 29-45. doi: 10.1016/j.fgb.2013.07.007 PMID: 23892063

8. Kowalczyk JE, Gruben BS, Battaglia E, Wiebenga A, Majoor E, de Vries RP. Genetic Interaction of Aspergillus nidulans galR, $x \ln R$ and $\operatorname{araR}$ in Regulating D-Galactose and L-Arabinose Release and Catabolism Gene Expression. PLoS One. 2015; 10: e0143200. doi: 10.1371/journal.pone.0143200 PMID: 26580075 9. De Ferreira Oliveira JMP, Van Passel MWJ, Schaap PJ, De Graaff LH. Shotgun proteomics of Aspergillus niger microsomes upon D-xylose inductions. Appl Environ Microbiol. 2010; 76: 4421-4429. doi: 10. 1128/AEM.00482-10 PMID: 20453123

10. Mohnen D. Pectin structure and biosynthesis. Curr Opin Plant Biol. 2008; 11: 266-277. doi: 10.1016/j. pbi.2008.03.006 PMID: 18486536

11. Niu J, Homan TG, Arentshorst M, de Vries RP, Visser J, Ram AFJ. The interaction of induction and repression mechanisms in the regulation of 
galacturonic acid-induced genes in Aspergillus niger. Fungal Genet Biol. 2015; 82: 32-42. doi: 10.1016/j.fgb.2015.06.006 PMID: 26127014

12. Martens-Uzunova ES, Schaap PJ. Assessment of the pectin degrading enzyme network of Aspergillus niger by functional genomics. Fungal Genet Biol. 2009; $46 \quad$ Suppl 1: S170-S179. Available: http://www. ncbi.nlm.nih.gov/pubmed/19618506

13. Tamayo-Ramos JA, Flipphi M, Pardo E, Manzanares P, Orejas M. Lrhamnose induction of Aspergillus nidulans $\alpha$-L-rhamnosidase genes is glucose repressed via a CreA-independent mechanism acting at the level of inducer uptake. Microb Cell Fact. 2012; 11: 26. doi: 10.1186/1475-2859-11-26 PMID: 22353731

14. Hilditch S, BerghaÈ 1l S, Kalkkinen N, PenttilaÈ M, Richard P. The missing link in the fungal D-galacturonate pathway: identification of the L-threo-3deoxy-hexulosonate aldolase. J Biol Chem. 2007; 282: 26195-201. doi: 10.1074/jbc.M704401200 PMID: 17609199

15. Zhang L, Thiewes $\mathrm{H}$, van Kan JAL. The D-galacturonic acid catabolic pathway in Botrytis cinerea. Fungal Genet Biol. 2011; 48: 990-7. doi: 10.1016/j.fgb.2011.06.002 PMID: 21683149

16. Koivistoinen OM, Arvas M, Headman JR, Andberg M, PenttilaÈ M, Jeffries $\mathrm{TW}$, et al. Characterization of the gene cluster for 1-rhamnose catabolism in the yeast Scheffersomyces (Pichia) stipitis. Gene. 2012; 492: 177-85. doi: 10.1016/j.gene.2011.10.031 PMID: 22037608

17. Martens-Uzunova ES, Schaap PJ. An evolutionary conserved d-galacturonic acid metabolic pathway operates across filamentous fungi capable of pectin degradation. Fungal Genet Biol. 2008; 45: 1449-57. doi: 10.1016/j.fgb.2008.08.002 PMID: 18768163

18. Gruben BS, Zhou M, Wiebenga A, Ballering J, Overkamp KM, Punt PJ, et al. Aspergillus niger RhaR, a regulator involved in L-rhamnose release and catabolism. Appl Microbiol Biotechnol. Springer Verlag; 2014; 98: 5531-5540. doi: 10.1007/s00253-014-5607-9 PMID: 24682478

19. Alazi E, Niu J, Kowalczyk JE, Peng M, Aguilar Pontes MV, van Kan JAL, et al. The transcriptional activator GaaR of Aspergillus niger is required for release and utilization of D-galacturonic acid from pectin. FEBS Lett. 2016; 20. Benz JP, Protzko RJ, Andrich JM, Bauer S, Dueber JE, Somerville CR. Identification and characterization of a galacturonic acid transporter from Neurospora crassa and its application for Saccharomyces cerevisiae fermentation processes. Biotechnol Biofuels. BioMed Central; 2014; 7: 20. doi: 10.1186/ 1754-6834-7-20 PMID: 24502254 
21. Sloothaak J, Schilders M, Schaap PJ, de Graaff LH. Overexpression of the Aspergillus niger GatA transporter leads to preferential use of D-galacturonic acid over D-xylose. AMB Express. Springer; 2014; 4: 66. doi: 10.1186/s13568014-0066-3 PMID: 25177540

22. Manzanares P, ValleÂs S, Ramòn D, Orejas M. $\alpha$-L-rhamnosidases: Old and New Insights. Industrial Enzymes. Dordrecht: Springer Netherlands; 2007. pp. 117-140.

23. Lahaye M, Robic A. Structure and functional properties of ulvan, a polysaccharide from green seaweeds. Biomacromolecules. 2007; 8: 1765-74. doi: 10.1021/bm061185q PMID: 17458931

24. Parakkottil Chothi M, Duncan GA, Armirotti A, Abergel C, Gurnon JR, Van Etten JL, et al. Identification of an L-rhamnose synthetic pathway in two nucleocytoplasmic large DNA viruses. J Virol. 2010; 84: 8829 \pm 38 . doi: 10.1128/JVI.00770-10 PMID: 20538863

25. Jack DL, Yang NM, Saier MH. The drug/metabolite transporter superfamily. Eur J Biochem. 2001; 268: 3620 39 . Available: http://www.ncbi.nlm.nih.gov/pubmed/11432728 PMID: 11432728

26. Rautengarten C, Ebert B, Moreno I, Temple H, Herter T, Link B, et al. The Golgi localized bifunctional UDP-rhamnose/UDP-galactose transporter family of Arabidopsis. Proc Natl Acad Sci. 2014; 111: 11563-11568. doi: 10.1073/pnas.1406073111 PMID: 25053812

27. Leandro MJ, Fonseca C, GoncËalves P. Hexose and pentose transport in ascomycetous yeasts: an overview. FEMS Yeast Res. 2009; 9: 511-25. doi: 10.1111/j.1567-1364.2009.00509.x PMID: 19459982

28. Reifenberger E, Boles E, Ciriacy M. Kinetic Characterization of Individual Hexose Transporters of Saccharomyces Cerevisiae and their Relation to the Triggering Mechanisms of Glucose Repression. Eur J Biochem. 1997; 245: 324333. PMID: 9151960

29. Wieczorke R, Krampe S, Weierstall T, Freidel K, Hollenberg CP, Boles E. Concurrent knock-out of at least 20 transporter genes is required to block uptake of hexoses in Saccharomyces cerevisiae. FEBS Lett. 1999; 464: 123-128. PMID: 10618490

30. Vankuyk PA, Diderich JA, MacCabe AP, Herrero O, Ruijter GJG, Visser J. Aspergillus niger mstA encodes a high-affinity sugar/H+ symporter which is regulated in response to extracellular pH. Biochem J. 2004; 379: 375-383. doi: 10.1042/BJ20030624 PMID: 14717659

31. Polidori E, Ceccaroli P, Saltarelli R, Guescini M, Menotta M, Agostini D, et al. Hexose uptake in the plant symbiotic ascomycete Tuber borchii Vittadini: 
biochemical features and expression pattern of the transporter TBHXT1. Fungal Genet Biol. 2007; 44: 187-198. doi: 10.1016/j.fgb.2006.08.001 PMID: 17005424 32. Saloheimo A, Rauta J, Stasyk O V., Sibirny A a., PenttilaÈ M, Ruohonen L. Xylose transport studies with xylose-utilizing Saccharomyces cerevisiae strains expressing heterologous and homologous permeases. Appl Microbiol Biotechnol. 2007; 74: 1041-1052. doi: 10.1007/s00253-006-0747-1 PMID: 17180689

33. Leandro MJ, SychrovaÂ H, Prista C, Loureiro-Dias MC. ZrFsy1, a highaffinity fructose $/ \mathrm{H}+$ symporter from fructophilic yeast Zygosaccharomyces rouxii. PLoS One. 2013; 8: e68165. doi: 10.1371/journal. pone.0068165 PMID: 23844167

34. Reis TF Dos, Menino JF, Bom VLP, Brown NA, Colabardini AC, Savoldi $\mathrm{M}$, et al. Identification of glucose transporters in Aspergillus nidulans. PLoS One. 2013; 8: e81412. doi: 10.1371/journal.pone. 0081412 PMID: 24282591

35. Du J, Li S, Zhao H. Discovery and characterization of novel d-xylose-specific transporters from Neurospora crassa and Pichia stipitis. Mol Biosyst. 2010; 6: 2150-2156. doi: 10.1039/c0mb00007h PMID: 20714641

36. Sloothaak J, Odoni DI, de Graaff LH, Martins Dos Santos VAP, Schaap PJ, Tamayo-Ramos JA. Aspergillus niger membrane-associated proteome analysis for the identification of glucose transporters. Biotechnol Biofuels. 2015; 8: 150. doi: 10.1186/s13068-015-0317-9 PMID: 26388937

37. Nordberg H, Cantor M, Dusheyko S, Hua S, Poliakov A, Shabalov I, et al. The genome portal of the Department of Energy Joint Genome Institute: 2014 updates. Nucleic Acids Res. 2014; 42: D26-31. doi: 10.1093/nar/gkt1069 PMID: 24225321

38. Gunn FJ, Tate CG, Henderson PJ. Identification of a novel sugar-H+ symport protein, FucP, for transport of L-fucose into Escherichia coli. Mol Microbiol. 1994; 12: 799-809. Available: http://www.ncbi.nlm. nih.gov/pubmed/8052131 PMID: 8052131

39. Paulsen IT, Chauvaux S, Choi P, Saier MH. Characterization of glucosespecific catabolite repression resistant mutants of Bacillus subtilis: identification of a novel hexose:H+ symporter. J Bacteriol. 1998; 180: 498-504. Available: http://www.pubmedcentral.nih.gov/articlerender.fcgi?artid=106914\&tool= pmcentrez\&rendertype $=$ abstract PMID: 9457850

40. Christensen M, Borza T, Dandanell G, Gilles A-M, Barzu O, Kelln RA, et al. Regulation of expression of the 2-deoxy-D-ribose utilization regulon, deoQKPX, from Salmonella enterica serovar typhimurium. J Bacteriol. 2003; 185: 6042-50. Available: $\quad$ http://www.pubmedcentral.nih.gov/articlerender.fcgi?artid= 
225019\&tool=pmcentrez\&rendertype $=$ abstract $\quad$ doi: $10.1128 / \mathrm{JB} .185 .20 .6042-$ 6050.2003 PMID: 14526015

41. Pao SS, Paulsen IT, Saier MH. Major facilitator superfamily. Microbiol Mol Biol Rev. 1998; $\quad 62: \quad 1 \pm 34 . \quad$ Available: http://www.pubmedcentral.nih.gov/articlerender.fcgi?artid=98904\&tool= pmcentrez\&rendertype=abstract PMID: 9529885 42. Psakis G, Saidijam M, Shibayama K, Polaczek J, Bettaney KE, Baldwin JM, et al. The sodiumdependent D-glucose transport protein of Helicobacter pylori. Mol Microbiol. 2009; 71: 391 \pm 403 . doi: 10.1111/j.1365-2958.2008.06535.x PMID: 19161491

43. Tate CG, Muiry JA, Henderson PJ. Mapping, cloning, expression, and sequencing of the rhaT gene, which encodes a novel L-rhamnose-H+ transport protein in Salmonella typhimurium and Escherichia coli. J Biol Chem. 1992; 267: 6923-32. Available: http://www.ncbi.nlm.nih.gov/pubmed/1551902 PMID: 1551902

44. Saier MH. Families of transmembrane sugar transport proteins. Mol Microbiol. 2000; 35: 699-710. Available: http://www.ncbi.nlm.nih.gov/pubmed/10692148 PMID: 10692148

45. Hong S-B, Lee M, Kim D-H, Varga J, Frisvad JC, Perrone G, et al. Aspergillus luchuensis, an industrially important black Aspergillus in East Asia. PLoS One. 2013; 8: e63769. doi: 10.1371/journal.pone. 0063769 PMID: 23723998

46. de Vries RP, Visser J, de Graaff LH. CreA modulates the XlnR-induced expression on xylose of Aspergillus niger genes involved in xylan degradation. Res Microbiol. 1999; 150: 281-5. Available: http:// www.ncbi.nlm.nih.gov/pubmed/10376490 PMID: 10376490

47. Sloothaak J, Tamayo-Ramos JA, Odoni DI, Laothanachareon T, Derntl C, Mach-Aigner AR, et al. Identification and functional characterization of novel xylose transporters from the cell factories Aspergillus niger and Trichoderma reesei. Biotechnol Biofuels. BioMed Central; 2016; 9: 148. doi: 10.1186/s13068016-0564-4 PMID: 27446237

48. Young EM, Comer AD, Huang H, Alper HS. A molecular transporter engineering approach to improving xylose catabolism in Saccharomyces cerevisiae. Metab Eng. 2012; 14: 401-11. doi: 10.1016/j.ymben. 2012.03.004 PMID: 22445945

49. Ferreira D, Nobre A, Silva ML, Faria-Oliveira F, Tulha J, Ferreira C, et al. $\mathrm{XYLH}$ encodes a xylose/H+ symporter from the highly related yeast species Debaryomyces fabryi and Debaryomyces hansenii. FEMS Yeast Res. 2013; 13: 585-96. doi: 10.1111/1567-1364.12061 PMID: 23809840 
50. Mishra NK, Chang J, Zhao PX. Prediction of membrane transport proteins and their substrate specificities using primary sequence information. PLoS One. 2014; 9: e100278. doi: 10.1371/journal.pone. 0100278 PMID: 24968309

51. Pardo E, Orejas M. The Aspergillus nidulans $\mathrm{Zn}$ (II)2Cys6 transcription factor AN5673/RhaR mediates L-rhamnose utilization and the production of $\alpha$-Lrhamnosidases. Microb Cell Fact. 2014; 13: 161. doi: 10.1186/s12934-014-01619 PMID: 25416526

52. Becker J, Boles E. A Modified Saccharomyces cerevisiae Strain That Consumes 1-Arabinose and Produces Ethanol. Appl Environ Microbiol. 2003; 69: 4144-4150. doi: 10.1128/AEM.69.7.4144-4150.2003 PMID: 12839792

53. Tantirungkij M, Nakashima N, Seki T, Yoshida T. Construction of xyloseassimilating Saccharomyces cerevisiae. J Ferment Bioeng. 1993; 75: 83 \pm 88 .

54. KoÈ tter P, Ciriacy M. Xylose fermentation by Saccharomyces cerevisiae. Appl Microbiol Biotechnol. 1993; 38: 776 783.

55. Wahl R, Wippel K, Goos S, KaĖmper J, Sauer N. A novel high-affinity sucrose transporter is required for virulence of the plant pathogen Ustilago maydis. PLoS Biol. 2010; 8.

56. Subtil T, Boles E. Improving L-arabinose utilization of pentose fermenting Saccharomyces cerevisiae cells by heterologous expression of L-arabinose transporting sugar transporters. Biotechnol Biofuels. BioMed Central Ltd; 2011; 4: 38 .

57. Colabardini AC, Nicolas L, Ries A, Brown NA, Fernanda T, Savoldi M, et al. Functional characterization of a xylose transporter in Aspergillus nidulans. Biotechnol Biofuels. 2014; 7: 46. doi: 10.1186/1754- 6834-7-46 PMID: 24690493

58. van der Veen D, Oliveira JM, van den BergWa M, de Graaff LH. Analysis of variance components reveals the contribution of sample processing to transcript variation. Appl Environ Microbiol. 2009; 75: 2414-22. doi: 10.1128/AEM.02270-08 PMID: 19233957

59. Ruijter GJ, Vanhanen SA, Gielkens MM, van de Vondervoort PJ, Visser J. Isolation of Aspergillus niger creA mutants and effects of the mutations on expression of arabinases and L-arabinose catabolic enzymes. Microbiology. 1997; 143 (Pt 9: 2991-8).

60. Ademark P, de Vries RP, HaÈgglund P, Stålbrand H, Visser J. Cloning and characterization of Aspergillus niger genes encoding an alpha-galactosidase and a beta-mannosidase involved in galactomannan degradation. Eur J Biochem. 2001; 268: 2982-90. Available: http://www.ncbi.nlm.nih.gov/pubmed/ 11358516 PMID: 11358516 
61. Cubero B, Gomez D, Scazzocchio C. Metabolite Repression and Inducer Exclusion in the Proline Utilization Gene Cluster of Aspergillus nidulans. J Bacteriol. 2000; 182: 233-235. PMID: 10613888

62. Forment J V., Flipphi M, RamoÂn D, Ventura L, MacCabe AP. Identification of the $m s t E$ gene encoding a glucose-inducible, low affinity glucose transporter in Aspergillus nidulans. J Biol Chem. 2006; 281: 8339-8346. doi: 10.1074/jbc.M508198200 PMID: 16418173

63. Forment J V., Flipphi M, Ventura L, GonzaÂlez R, RamoÂn D, MacCabe AP. High-affinity glucose transport in Aspergillus nidulans is mediated by the products of two related but differentially expressed genes. PLoS One. Public Library of Science; 2014; 9: e94662. doi: 10.1371/journal.pone.0094662 PMID: 24751997

64. Galazka JM, Tian C, Beeson WT, Martinez B, Glass NL, Cate JHD. Cellodextrin Transport in Yeast for Improved Biofuel Production. Science. 2010; 330 (6000):84-6. doi: 10.1126/science.1192838 PMID: 20829451

65. Zhang W, Kou Y, Xu J, Cao Y, Zhao G, Shao J, et al. Two major facilitator superfamily sugar transporters from Trichoderma reesei and their roles in induction of cellulase biosynthesis. J Biol Chem. 2013; 288: 32861-32872. doi: 10.1074/jbc.M113.505826 PMID: 24085297

66. Huang Z-B, Chen X-Z, Qin L-N, Wu H-Q, Su X-Y, Dong Z-Y. A novel major facilitator transporter TrSTR1 is essential for pentose utilization and involved in xylanase induction in Trichoderma reesei. Biochem Biophys Res Commun. 2015; 460: 663-9. doi: 10.1016/j.bbrc.2015.03.087 PMID: 25817789

67. Edwards MC, Doran-Peterson J. Pectin-rich biomass as feedstock for fuel ethanol production. Appl Microbiol Biotechnol. Springer; 2012; 95: 565-75. doi: 10.1007/s00253-012-4173-2 PMID: 22695801

68. Behera S, Singh R, Arora R, Sharma NK, Shukla M, Kumar S. Scope of algae as third generation biofuels. Front Bioeng Biotechnol. Frontiers Media SA; 2014; 2: 90. doi: 10.3389/fbioe.2014.00090 PMID: 25717470

69. Bos CJ, Debets AJ, Swart K, Huybers A, Kobus G, Slakhorst SM. Genetic analysis and the construction of master strains for assignment of genes to six linkage groups in Aspergillus niger. Curr Genet. 1988; 14: 437-43. Available: http://www.ncbi.nlm.nih.gov/pubmed/3224384 PMID: 3224384

70. Carvalho NDSP, Arentshorst M, Jin Kwon M, Meyer V, Ram AFJ. Expanding the $k u 70$ toolbox for filamentous fungi: establishment of complementation vectors and recipient strains for advanced gene analyzes. Appl Microbiol Biotechnol. 2010; 87: 1463-73. doi: 10.1007/s00253-010-2588-1 PMID: 20422182 
71. Pontecorvo G, Roper JA, Chemmons LM, Macdonald KD, Bufton AWJ. The Genetics of Aspergillus nidulans. Advances in Genetics. 1953. pp. 141-238. PMID: 13040135

72. Vishniac W, Santer M. The thiobacilli. Bacteriol Rev. 1957; 21: 195-213. Available: http://www. pubmedcentral.nih.gov/articlerender.fcgi?artid $=180898 \&$ tool $=$ pmcentrez\&rend ertype $=$ abstract PMID: 13471458

73. Janicka-Russak M, Kabaøa K, Burzynski M. Different effect of cadmium and copper on $\mathrm{H}+$-ATPase activity in plasma membrane vesicles from Cucumis sativus roots. J Exp Bot. 2012; 63: 4133-42. doi: 10.1093/jxb/ers097 PMID: 22451724

74. Rajala N, Hensen F, Wessels HJCT, Ives D, Gloerich J, Spelbrink JN. Whole Cell Formaldehyde Cross- Linking Simplifies Purification of Mitochondrial Nucleoids and Associated Proteins Involved in Mitochondrial Gene Expression. PLoS One. 2015; 10: e0116726. doi: 10.1371/journal.pone.0116726 PMID: 25695250

75. Cox J, Mann M. MaxQuant enables high peptide identification rates, individualized p.p.b.-range mass accuracies and proteome-wide protein quantification. Nat Biotechnol. Nature Publishing Group; 2008; 26: 1367-72. doi: 10.1038/nbt.1511 PMID: 19029910

76. Gietz RD, Woods RA. Transformation of yeast by lithium acetate/singlestranded carrier DNA/polyethylene glycol method. Methods Enzymol. 2002; 350: 87-96. Available: http://www.ncbi.nlm.nih.gov/ pubmed/12073338 PMID: 12073338

77. Meyer V, Arentshorst M, El-Ghezal A, Drews AC, Kooistra R, van den Hondel $\mathrm{C}$ a, et al. Highly efficient gene targeting in the Aspergillus niger kusA mutant. JBiotechnol. 2007; 128: 770-775.

78. Kusters-van Someren MA, Harmsen JAM, Kester HCM, Visser J. Structure of the Aspergillus niger pelA gene and its expression in Aspergillus niger and Aspergillus nidulans. Curr Genet. 1991; 20: 293-299. PMID: 1934134

79. Mach-Aigner AR, Omony J, Jovanovic B, van Boxtel AJB, de Graaff LH. DXylose concentration-dependent hydrolase expression profiles and the function of CreA and XlnR in Aspergillus niger. Appl Environ Microbiol. 2012; 78: 3145 3155. doi: 10.1128/AEM.07772-11 PMID: 22344641

80. Arvidsson S, Kwasniewski M, Riaño-PachoÂn DM, Mueller-Roeber B. QuantPrime $<$ a flexible tool for reliable high-throughput primer design for quantitative PCR. BMC Bioinformatics. BioMed Central; 2008; 9: 465. doi: 10.1186/1471-2105-9-465 PMID: 18976492 
81. Walsh MC, Smits HP, Scholte M, van Dam K. Affinity of glucose transport in Saccharomyces cerevisiae is modulated during growth on glucose. J Bacteriol. 1994; 176: 953-8. Available: http://www. pubmedcentral.nih.gov/articlerender.fcgi?artid=205144\&tool=pmcentrez\&rend ertype=abstract PMID: 8106337

82. Krogh A, Larsson B, von Heijne G, Sonnhammer EL. Predicting transmembrane protein topology with a hidden Markov model: application to complete genomes. J Mol Biol. 2001; 305: 567-80. doi: 10.1006/ jmbi.2000.4315 PMID: 11152613

83. Simossis VA, Heringa J. PRALINE: a multiple sequence alignment toolbox that integrates homology extended and secondary structure information. Nucleic Acids Res. 2005; 33: W289-94. doi: 10.1093/ nar/gki390 PMID: 15980472

84. Petersen TN, Brunak S, von Heijne G, Nielsen H. SignalP 4.0: discriminating signal peptides from transmembrane regions. Nat Methods. Nature Publishing Group, a division of Macmillan Publishers Limited. All Rights Reserved.; 2011; 8: 785-6. doi: 10.1038/nmeth.1701 PMID: 21959131 


\section{Chapter 7}

\section{General discussion}

Jasper Sloothaak 


\section{General discussion}

\section{Structure, mechanism and function of MFS sugar transporters}

In this thesis the identification and characterization of ten sugar transporters belonging to Major Facilitator Superfamily (MFS) families 2.A.1.1 (the Sugar Porter (SP) family), and 2.A.1.7 (the Fucose: $\mathrm{H}^{+}$Symporter (FHS) family) are presented. In this chapter I will discuss the most important structural features of these transporter families and how they function. MFS proteins transport various compounds such as sugars, drugs, amino acids and alcohols across cellular membranes, and prokaryotic and are widespread among all forms of life. In all cells types, they play an important role in the import of carbon and energy sources and export of toxic compounds, and in mammals specifically they play a role in cell-cell communication and distribution of compounds between various tissues. Upon binding of the substrate, MFS transporters undergo a series of conformational changes as reviewed by Quistgaard et al., 2016 [1]. To fully understand the mechanism of these changes, a deep understanding of the tertiary protein structure is required. Since the first MFS crystal structure was published of LacY in 2003, many MFS families now have a representative crystal structure solved. Fortunately, of the 2.A.1.1 SP family, structures are solved in several different conformations ( $\mathrm{LacY}$ and XylE), making it possible to draw conclusions on the mechanism of action. The study of these various conformational states with- and without bound substrate has led to the formulation of a mechanistic model that has recently been updated [1]. Furthermore, using homology modeling on crystal structures in combination with these models, researchers were able to pinpoint specific functional residues, involved in substrate binding, anion binding, gating or eliciting conformational changes. Here I will discuss these mechanisms and functional residues, as these studies also provide insights for the development of tailor-made synthetic transporters tuned towards the specifics of second generation feedstocks. Furthermore, I will discuss some novel insights on the regulatory mechanisms that are involved in nutrient uptake.

\section{Structural basis for transport; the 'clamp-and-switch' model}

As already mentioned in chapter 2, the MFS transporters have one of three mechanisms of action. They can function as uniporters, where a metabolite is transported over the membrane, driven by its concentration gradient. They can function as antiporters, where two metabolites are transported over the membrane in opposite direction, driven by the concentration gradient of one of the metabolites. They can also function as symporters, where two metabolites are 
transported over the membrane in the same direction, driven by the concentration gradient of one if the two metabolites. These transporters can be divided based on secondary structure predictions for transmembrane helices (TMHs) and loops that connect the TMHs and are found at the terminal ends of the proteins. Based on evolutionary and homology studies, the twelve TMHs in MFS transporters are subdivided in two times six TMHs in the $\mathrm{N}$-terminal and $\mathrm{C}$-terminal domains. Those bundles of six TMHs are subdivided into two inverted repeats of $3 \mathrm{TMHs}$ [2]. After the discovery of LacY, when it became evident that (single) proteins were responsible for solute transport, the alternative access model was formulated [3]. The model postulated that the transporter protein that is spanning the membrane, changes conformation upon binding of the substrate, exposing the substrate binding site to the cytoplasm with a changed binding affinity as depicted in figure 1. This hypothesis has since been confirmed and expanded using various biochemical and biophysical experimental approaches using the model transporter LacY to develop the approaches, as outlined in [4]. Also, many crystal structures have been solved that give physical evidence to the predicted structures of these proteins, and even full cysteine scanning has been performed to identify all crucial residues [5]. The 3dimensional structure of the sugar transporters LacY and XylE have even been crystallized in more than one conformation. This abundance of experimental and structural data has led to the expansion of the alternating access model in the rocker-switch mechanistic model. In all structures, we find the pseudo-symmetrical Nand C-terminal 6-helix domains that each consist two 3-helix inverted repeats [6]. The alternating access model dictates that the substrate enters the hydrophilic cleft between these two 6-THM domains from the periplasmic space and is bound by the substrate binding residues that reside in the deepest part of the cleft. The rocker-switch model postulates that the rigid domains then move through 'rocking', with the substratebinding site as the rotation axis. In crystal structures of the inward open conformations, the middle helices of the previously mentioned 3-helix inverted repeats (helix 2, 5, 8 and 11)

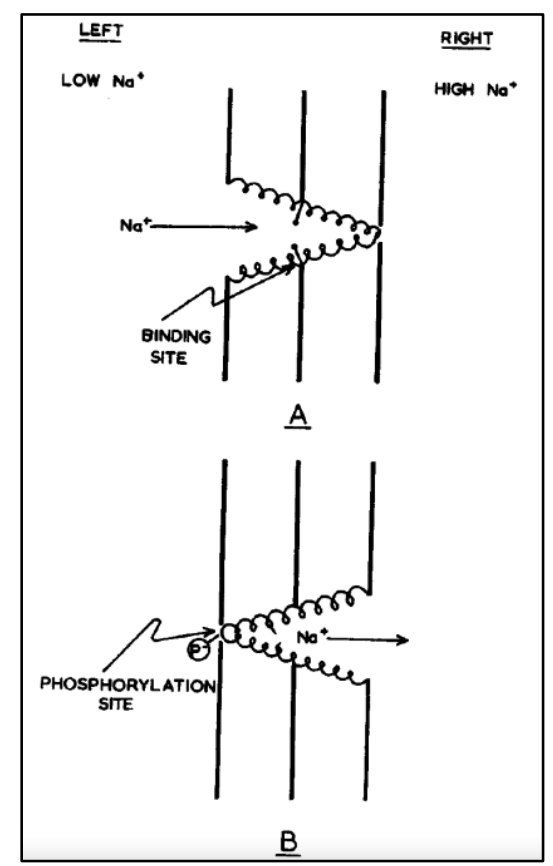

Figure 1 Drawing of the alternative access model by Jardetsky et al. 
are curved, and retain this structure in the outward open state. This shape is used to switch between conformations, interchanging inverted topology repeats [6]. The formation of interactions between the periplasmic domains then results in the closing of the periplasmic cleft and the loss of interactions between the cytoplasmic domains results in the opening of the cytoplasmic cleft, exposing the substrate to the other side of the membrane and vice versa. The two conformations described in this model are indeed found in crystal structures such as the inward open LacY of Abramson and the outward open FucP of Dang, 2010 [7][8]. Conformations between the inward and the outward open conformations, however, have also been captured in resolved crystal structures for MFS transporters. These structures display a range of 'occluded' states, where the binding site is inaccessible from either side of the membrane. These conformations are not possible if we assume the previously mentioned curved shape of the B-helices with full rigidness of the two ( $\mathrm{N}$ - and $\mathrm{C}-$ ) domains. The first helices of the 3-helix inverted repeats (helix 1, 4, 7 and 10; see figure 2) are bend in occluded states in contrast to the opened states. The bending of these helices forms a clamp on the opened side of the substrate-binding pocket and forms the occluded state that can clearly be distinguished as an inward-open occluded or outward-open occluded state. After formation of the clamp, the protein switches to the other conformation with the rocking movement. So a transport cycle goes: binding of the substrate; bending of the A-helices to form the clamp; rocker-switch movement with B-helices as axis; release of clamp on opposite side with the subsequent release of the substrate. The clamp-and-switch

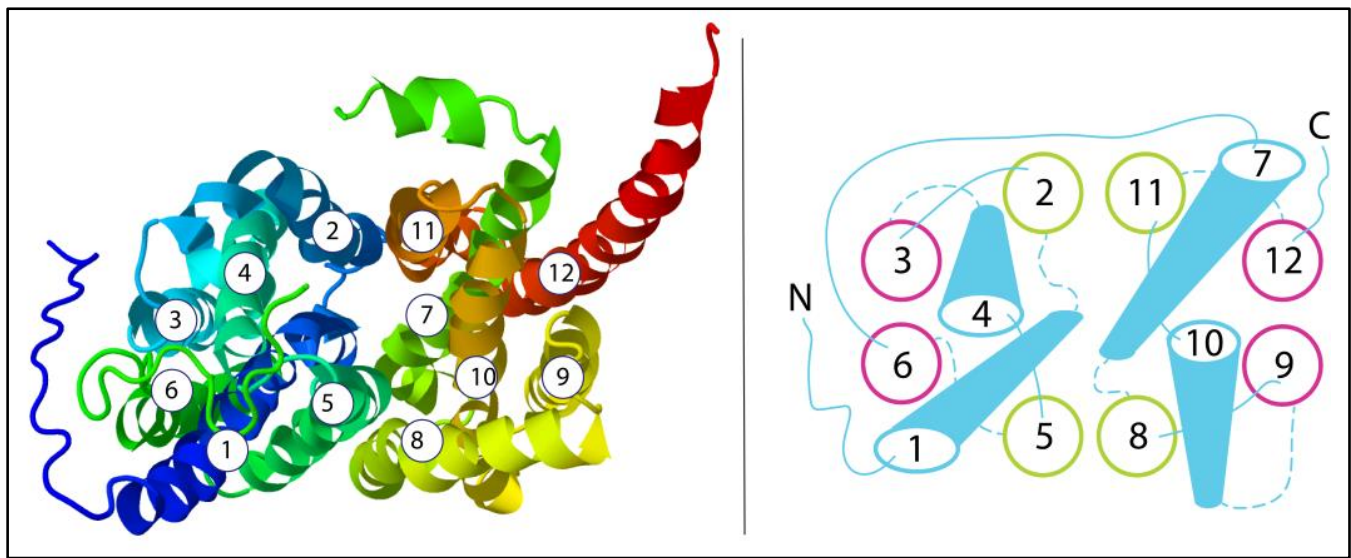

Figure 2 Two different schematic representations of the 12 transmembrane helices of the FucP protein 
mechanism is the mechanism of action for the novel proteins of the SP and FHS families that are identified in this thesis. Besides substrate binding and specific conformational changes, these transporters also have domains functioning as gates that influence substrate specificity [9]. Furthermore, these gating residues are responsible for the transient interactions that occur between the N- and Cterminal domains during the transport cycle. The best-understood gating motif is the motif-A, responsible for closing the cytoplasmic gate and a key motif in all MFS transporters [10][1].

\section{Chemical basis for transport; binding pocket and gating elements as a basis for transporter bioengineering}

In reverse genetics, a gene sequence is specifically engineered to study the phenotypic effect of this change. It is regularly applied to identify functional residues that are responsible for the biochemical characteristics of transporters. In several cases, whole-protein cys-scanning has been performed, while in other cases random chimera proteins have led to the identification of crucial residues $[5,11]$. Yet another approach is the introduction of random mutations with subsequent sequencing of functionally interesting variants. Using these approaches, many functional residues were coupled to substrate or $\mathrm{H}+$ binding in several transporters. However, the sequence identity of MFS transporters is typically as low as $15 \%$, and functional regions determining substrate specificity only align for very closely related proteins, as discussed in chapter 4 and previously by Kasho et al. in 2006 [12]. However, in functional genome-based screens they are easily recognized as MFS, because secondary structures are very well conserved. The transporters that were identified in this thesis are part of the Sugar Porters (SP) family and the Fucose: $\mathrm{H}^{+}$Symporter (FHS) family, that are represented by the well-studied XylE and FucP of E. coli respectively. A thorough study has been done to determine functional residue correspondence between these transporters. These studies have led to a proposed model that explains the evolutionary divergence between these transporters by the intragenic rearrangement of 3-TMH units [13]. This theory was, however, soon disputed with compelling arguments $[2,14]$. It can be concluded that identification of functional residues for a sugar transporter of one family cannot be extrapolated to transporters from other families, but it is likely that it is feasible within families. In table 1 putative functional residues and motifs of the novel transporters of this thesis are summarized, extracted from an inter-family primary sequence alignment. For the SP-family protein XylE, an in-depth analysis has been done on functional residues upon solving the crystal structure in several conformations, binding xylose or glucose $[10,15,16]$. Many residues, shown to 
be involved in substrate binding, gating and proton binding have been identified. These residues showed a high, but not a strict level conservation in the transporters presented in this thesis. In figure 3, XylE is represented in the cytoplasmic membrane and the functional residues that were identified are highlighted. Furthermore, the three intracellular helices between TMH 6 and 7, which pack into a bundle against residues 463-479 to cap the cytoplasmic side in the outward open state and swing away in the inward open state, are indicated with yellow [10]. The TMHMM tool was used to predict the transmembrane segments represented, while the helices in the intracellular loops are derived from

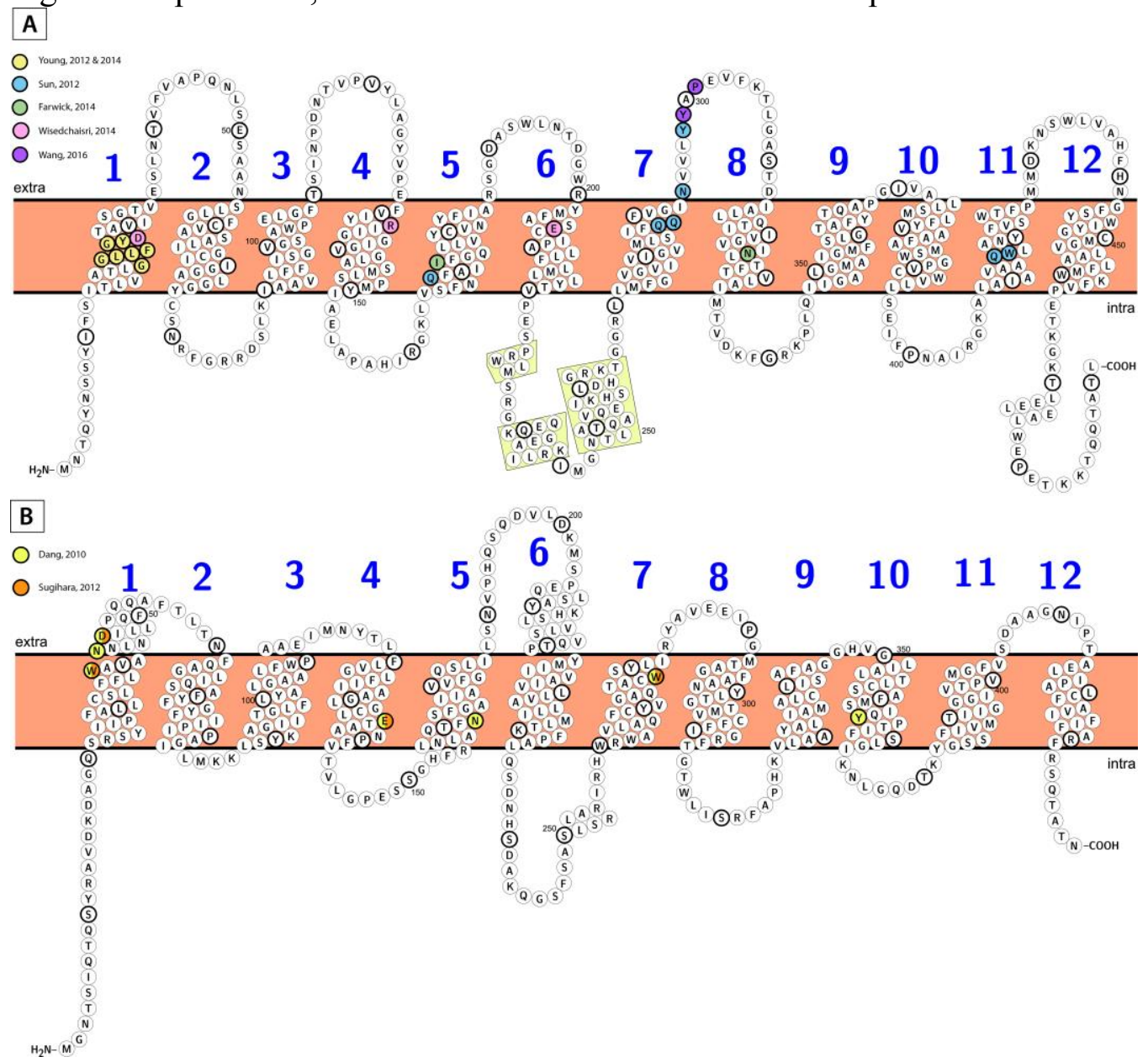

Figure 3 Schematic representation of the model transporters XylE (A) and FucP (B). Identified functional residues are color-coded according to reference. TMH predictions are done with TMHMM 
the crystal structures of Sun et al. In figure 3, the residues involved in binding can be seen in their spatial conformation while binding Xylose (A) or Glucose (B). While considering binding residues, it must be noted that lack of transport does not mean lack of binding. In the previous section, it was discussed that transport requires binding and subsequent conformational change. A substrate that is structurally similar may bind but not induce a conformational change, resulting in transport inhibition. It is generally accepted that co-utilization of xylose and glucose will be an imported feature for the feasibility of renewable biochemical production, but this preferred substrate problem must first be addressed [17]. This problem is particularly important in the case of xylose transporters, that in most cases are inhibited by glucose [18]. However, in the studies presented in this thesis some very interesting exceptions have been discovered. In chapter 3 and 4, the uptake of glucose and xylose by newly identified transporters was studied, some of them displaying unique particularities in terms of substrate specificity, are addressed. In one particular experiment, where the transporters are expressed in a xylose-utilizing yeast strain, it is found that MstG transports glucose but not xylose and therefore does not support growth on xylose (Chapter 3, Figure 3) Similarly, XltB, like XylE, transports xylose but not glucose and therefore does not support growth on glucose (Chapter 4, Figure 4). MstG displays minor inhibition of glucose transport in the presence of 10-fold excess of xylose (Chapter 3, Figure 6). While for XltB, we found growth inhibition in the presence of glucose (Unpublished results). In both cases, this suggests that there is binding without subsequent conformational change that would result in transport of the (alternative) substrate over the membrane. These two striking exceptions can give an indication of functional residues, involved in the binding and transport of either substrate, therefore, I hereby discuss some of the residues, that may be important for xylose and glucose, but also other sugars.

Previously, a substitution of a residue was found in Saccharomyces. cerevisiae Gal2 and HXT36, corresponding to XylE N325, which can relieve the glucose inhibition $[18,19]$. In our transporters, we found the substitution of this asparagine by glycine in 4 cases, XltA, Str1 and 2, and GatA. It was not determined, however, if this amino-acid substitution results in relieve of transport inhibition by glucose. To determine exactly which residues are responsible for the discrimination between xylose and glucose, specific mutants should be tested. The residues identified previously, summarized in Table 1, provide a starting point for this analysis. As an example, the glutamate at position 189 of the XltB transporter is unique in this position and could, therefore, be in part responsible for the selectivity, so mutation to glutamine would confirm or reject this 
hypothesis. Also, the valine at position 194 of the MstG transporter is unique in this position and could, therefore, be in part responsible for the selectivity, so mutation to isoleucine would confirm or reject this hypothesis. Also, in our transporters, one of the residues responsible for proton binding in E. coli XylE (residue D27), is not conserved for XltA and Str1. This could mean that the proton symport mechanism is altered or not functional for these transporters.

\section{Design of the perfect xylose transporter}

As mentioned before, native fungal transporters have a natural preference for the use of glucose over xylose. As a result, the transport of alternative carbon sources such as xylose is inhibited when glucose is present. With the current technology and knowledge it is possible to engineer transporters and adjust their substrate affinities. Several successful attempts have been made to adjust the transport affinities and velocities for xylose and glucose, which have led to interesting insights about functional residues. The functions of transporters are described with the kinetic parameters $\mathrm{Km}$ and Vmax and roughly follow monod kinetics. When improvements are done on a transporter we are optimizing these parameters. Ideally, we want to build a transporter with a high capacity or speed, Vmax, and we want the transporter to have a high affinity, or low $\mathrm{Km}$, so it will function at this high transport velocities even when low amounts of substrate is available. Most importantly, we also want to the transporter to import xylose without the competitive inhibition of glucose. In a study by Farwick et al., two residues were identified that have a crucial effect on the competitive inhibition by glucose [18]. These two residues are not directly involved in binding of the substrate, but have a more subtle effect on the positioning and charge of the neighbouring residues that are involved in binding. Even, changing the amino acids that did directly bind the substrate resulted in most cases in a loss-of function mutant. The residues that function in the binding of glucose that were expected to be crucial in the selectivity are known and have been the target of

Table 1: Putative functional residues of the transporters identified in this thesis. Identified by inter-family primary sequence alignment

\begin{tabular}{|c|c|c|c|c|c|c|c|c|c|c|c|c|c|c|c|c|c|c|c|c|c|c|c|c|c|}
\hline & \multicolumn{6}{|c|}{ Young, 2012/2014 } & \multicolumn{2}{|c|}{ Farwick, 2014} & \multicolumn{6}{|c|}{ Reznicek, 2015} & \multicolumn{11}{|c|}{ Nijland, 2014} \\
\hline & \multicolumn{14}{|c|}{ gal2 residue \# } & \multicolumn{11}{|c|}{ hxt36 residue } \\
\hline & 81 & 82 & 83 & 84 & 85 & 86 & 219 & 376 & 301 & 310 & 311 & 314 & 386 & 435 & 206 & 332 & 333 & 337 & 338 & 341 & 367 & 437 & 441 & 446 & 469 \\
\hline Mst G & G & G & $\mathrm{L}$ & $\mathrm{L}$ & $\mathrm{F}$ & G & $T$ & $\mathrm{~N}$ & $E$ & - & - & $S$ & $T$ & M & $Q$ & $Q$ & $Q$ & $S$ & $\mathrm{~N}$ & $F$ & $\mathrm{~N}$ & $Y$ & w & w & $\mathrm{N}$ \\
\hline MstH & G & G & 1 & L & $\mathrm{F}$ & G & T & $\mathrm{N}$ & $\mathrm{E}$ & M & $\mathrm{S}$ & K & $M$ & L & Q & $Q$ & $Q$ & V & $\mathrm{N}$ & $\mathrm{F}$ & $\mathrm{N}$ & $\mathrm{F}$ & W & w & $\mathrm{N}$ \\
\hline XItA & G & G & $\mathrm{F}$ & v & $Y$ & G & $T$ & $E$ & $E$ & $E$ & $E$ & K & w & $T$ & Q & $Q$ & $Q$ & 1 & $\mathrm{~N}$ & $\mathrm{~N}$ & $E$ & $\mathrm{~F}$ & w & w & $\mathrm{N}$ \\
\hline XItB & G & G & $\mathrm{L}$ & $T$ & $\mathrm{~F}$ & G & $\mathrm{V}$ & Q & D & $Q$ & $E$ & $\mathrm{v}$ & $T$ & $\mathrm{~s}$ & $E$ & $Q$ & $Q$ & 1 & $\mathrm{~N}$ & 1 & $Q$ & $\mathrm{~F}$ & w & w & $\mathrm{N}$ \\
\hline XItC & G & G & G & L & $\mathrm{F}$ & G & $T$ & $\mathrm{~N}$ & $E$ & $R$ & Q & D & w & $\mathrm{v}$ & Q & $\mathrm{s}$ & $Q$ & $M$ & $\mathrm{~N}$ & $M$ & $\mathrm{~N}$ & $\mathrm{Y}$ & $\mathrm{F}$ & w & $\mathrm{N}$ \\
\hline Str1 & G & G & V & L & $Y$ & G & C & M & $\mathrm{E}$ & K & $R$ & A & W & A & Q & Q & Q & 1 & $\mathrm{~N}$ & $\mathrm{~L}$ & M & $\mathrm{F}$ & W & W & $\mathrm{N}$ \\
\hline Str2 & G & G & $F$ & L & $F$ & G & $T$ & $\mathrm{~N}$ & $A$ & $E$ & $S$ & $R$ & L & G & $Q$ & $Q$ & $Q$ & C & $\mathrm{N}$ & 1 & $\mathrm{~N}$ & $\mathrm{~F}$ & $F$ & w & $T$ \\
\hline Str3 & G & G & L & 1 & $F$ & G & $T$ & $\mathrm{~N}$ & $E$ & D & K & $T$ & $M$ & L & $Q$ & $Q$ & $Q$ & A & $\mathrm{N}$ & $\mathrm{F}$ & $\mathrm{N}$ & $\mathrm{F}$ & W & W & $\mathrm{N}$ \\
\hline GatA & G & $S$ & L & L & $\mathrm{F}$ & G & V & K & $E$ & V & A & $E$ & V & $M$ & Q & $Q$ & $Q$ & $T$ & $\mathrm{~N}$ & G & K & $Y$ & W & W & $Q$ \\
\hline
\end{tabular}


many studies [20]. But these results suggests that mutations to these residues adversely affect the transporter function, while mutations in residues in the proximity of the binding pockets have led to this successful improvement as is also shown in another study [19]. Young et al. were able to improve two transporters by random evolution and selection [21]. For one of these transporters, mutants were obtained with up to an order of magnitude improved uptake velocity, but the increased Vmax in almost all cases came with a decreased affinity for the substrate. One exceptional mutant showed an increased affinity as well as an increased uptake rate, and surprisingly only had one single amino acid substitution in a terminal region of the protein. The adjustment of residues in terminal regions has in at least one other case also led to very successful improvement of xylose transporters [22]. These examples show that the functioning of transporters is still very poorly understood, and the greatest improvements have come from random mutation in combination with a welldesigned assay. Furthermore, although researchers have been able to classify transporters on the basis of their structure as described in the introduction, the function of only a minute fraction of all the natural transporter has is known to us. In this thesis we focussed on identifying the function of more of those transporters and have shown that transporters with desirable characteristics are definitely 'out there'. Big steps to genetic engineering of cell factories can still be made by mining the natural transportome, before we are ready to truly design custom transporters.

\section{Residues of the FHS transporters}

Regarding the transporters of the fucose: $\mathrm{H}^{+}$Symporter family (FHS), functional residues have also been proposed for the FucP transporter protein, upon solving the crystal structure. An in-depth analysis of the FHS-family protein FucP has been done on functional residues upon solving the crystal structure by [8]. Several functional residues have been identified that are responsible for the binding of the substrate, binding of the proton or the interactions between the Nand C-terminal domains. The proposed mechanism and residues were later partly confirmed by Tryptophan fluorescence quenching studies [23]. Several of the most important residues are conserved in our transporter such as the W38, involved in substrate binding, and D46 and E135, involved in proton binding and helix interactions. Several other residues that are crucial for FucP, have nonconservative substitutions, such as W278A. The conserved D46 of TMH 1 is proposed to be protonated, to let the substrate pass, then deprotonated by the conserved E135 of TMH 4 to interact with W278 of TMH7 and close the periplasmic side. To study this specific location, the sequence of RhtA was 
modeled on the FucP crystal structure 3O7Q. No tryptophan or amino acid of similar function can be found in the RhtA sequence close to this position, and thus apparently this interaction closing the periplasmic side does not exist for RhtA. On the cytoplasmic side, a similar situation can be found. The conserved E135 is proposed to interact with the W365 residue when not protonated, which is substituted by phenylalanine. Therefore, also this interaction between the Nand C-terminus is not conserved. The fucose crystal structure was solved in conjunction with $\beta$-nonylglucoside, making it impossible to judge the effect of these substitutions. However, it was expected that the binding cavity of the rhamnose transporter is different from the cavity of the fucose transporter due to the different plane of the methyl group and the $\gamma$-hydroxyl group. The mechanism of action, is expected to be similar, but cannot be formulated yet.

\section{Regulation of and by transporters}

Elaborate studies have been done to understand the lifecycle of Aspergillus species, from spore germination to reproduction. Most of the regulatory events that determine changes of the developmental stages of the fungus take place at transcriptional level, and the region upstream of the Tata-box of the responsible structural genes is a hot spot for the binding of activating and repressing elements. Many transcriptional regulators have been identified that interact in cascades to determine the fate of growing mycelium [24]. In the natural environment, fungi change their metabolism for germination, vegetative growth, sexual reproduction, non-sexual reproduction and dormancy in response to environmental factors such as moisture content, salt and mineral availability, the presence of carbon source, oxygen, other organism and even light. Because filamentous fungi are multicellular, the response within a colony can be spatially very diverse due to varying conditions. Gradients of signaling molecules are believed to be responsible for this heterogeneity, which is mimicked on lab studies on plate growth, but assumed to be absent in controlled submerged fermentations. The regulation is mediated by the RNA- and DNA-binding zincfinger transcription factors. For the study of polysaccharide degradation and (oligo-) saccharide transport, there are a number of transcription factors in $A$. niger that are of major relevance. CreA is believed to be the most important mediator for carbon catabolite repression (CCR), functionally characterized already in 1973 [25]. CreA has two zinc finger domains and is an example of the type I classical zinc finger proteins ubiquitously present in the $A$. niger genome [26]. CreA is activated by the presence of glucose, high concentrations of xylose and other repressing carbon sources [27]. It provokes direct and indirect repression of genes involved in carbon utilization through DNA binding in a 
sequence consensus motif. This repression capacity is enhanced by additional specific motifs located in close proximity [28]. The binding to these motifs in promoter regions represses the expression of a wide variety of genes that are involved in the utilization of alternative carbon sources to glucose. In addition to CreA, other transcription factors have been characterized that control the response to changes in environmental $\mathrm{pH}$, or salinity, but also more specific regulators controlling depolymerization, transport and metabolism of plant cell wall components. Regarding the latter, a very well characterized transcription factor for is XlnR, the xylanolytic regulator [29]. XlnR has only 1 zinc finger domain of the strictly fungal class III zinc binuclear cluster type. It is a master regulator that has analogs in many other fungal species such as the Xyrl in $T$. reesei. It interacts with other transcription factors such as the cellulose response factors ClbR, ClrA, ClrB and the Activators of Cellulase Expression ACE1 and ACE2, to control the response to lignocellulosic biomass substrate [30]. The relevant DNA binding motif has been studied in detail and a consensus sequence was determined [31]. Furthermore, it functions in conjunction with the arabinose response element AraR to control pentose metabolism and response to xylan biomass substrate [32]. More recently, regulators have also been identified that specifically mediate the response to pectin components, namely GaaR; for response to galacturonic acid, and RhaR; for response to rhamnose. For these regulators, binding sequences were also proposed [33,34]. These regulators all belong to the strictly fungal class III transcription factors of which 296 can be found in the A. niger genome (Pfam domain PF00172). The relevance of the CreA regulator for glucose transporters such as those identified in chapter 3, has been demonstrated in previous studies [35]. Similarly, the relevance of XlnR for the efficient utilization of hemicellulosic biomass has been demonstrated previously [36]. In chapter 4, however, it is demonstrated for the first time that $\mathrm{X} \operatorname{lnR}$ and $\mathrm{Xyr} 1$ are directly responsible and crucial for the induction of the functionally characterized xylose transporters XltA and Str1, a strong indication for a biological role of these transporters. In contrast, our studies suggest that in some cases sugar transporters able to specifically transport a type of monosaccharide are not controlled at transcriptional level by the corresponding regulator. We found that $\mathrm{X} \ln \mathrm{B}$ was only able to transport xylose of the many sugars tested in this study, but is not controlled at transcriptional level by XlnR. Parallel to the studies performed for this thesis, a transcription factor similar to $\mathrm{X} \operatorname{lnR}$ was identified, that mediates a response to galacturonic acid [34]. Therefore, an additional experiment was performed to show the relevance of this regulator $(\mathrm{GaaR})$ to the transporter (GatA) that was functionally validated in chapter 5. This transcriptional analysis was done as described in chapter 5 and 
confirms the findings of Alazi et al. In figure 4 it can clearly be seen that the expression of GatA is repressed in response to the presence of glucose and induced in response to the presence of galacturonic acid. This induction is abolished in the gaaR deletion strain, showing that the galacturonic acid regulator is responsible for induction of GatA in response to the presence of galacturonic acid. In a similar way, we demonstrated in chapter 6 that the expression of the rhamnose transporter RhtA is dependent on the presence of the RhaR regulator.

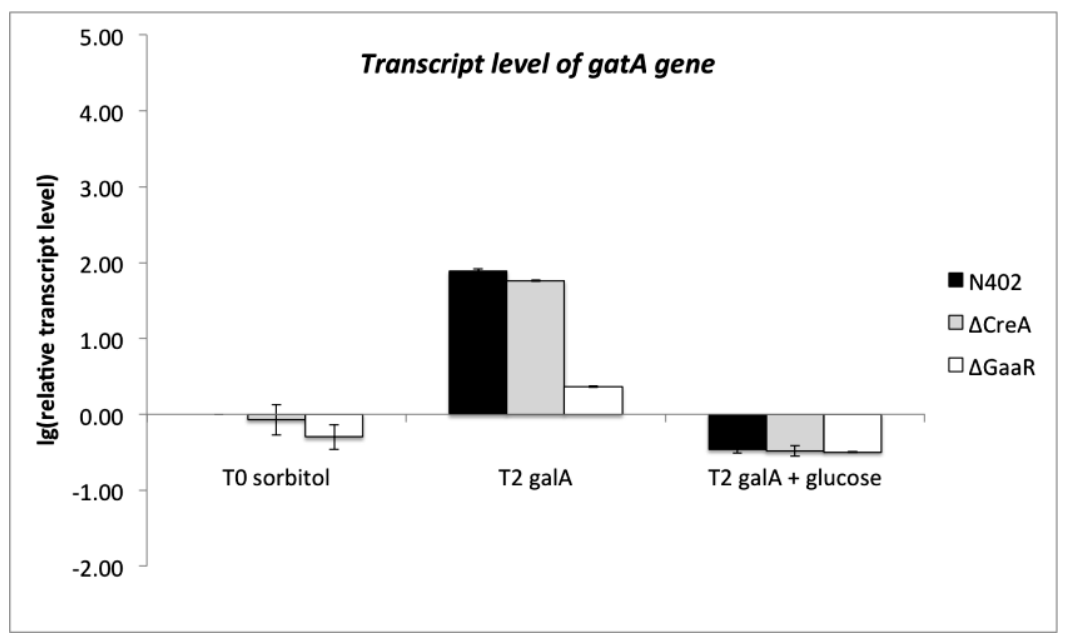

Figure 4 Transcript level of the GatA gene in 3 genetic backgrounds and 3 different carbon sources

\section{Sensing}

To understand how sugars are detected, proteins should be considered that are localized at the barrier of the periplasm and cytoplasm, namely in the cellular membrane, and have domains that interact with both.

In mammals, glucose sensing is a critical attribute, related to various metabolic dysfunctions and illnesses such as diabetes and is therefore well studied. An interesting system was discovered in pancreatic $\beta$-cells that govern the availability of insulin in mammalian blood. A constitutively expressed, highcapacity, low-affinity glucose transporter rapidly imports glucose that is subsequently sensed internally by the glucose-kinase system. In these dedicated cells, this leads to a cascade of reactions and ultimately to excretion of insulin in response to the extracellular glucose. Other studies provided evidence for the existence of an important glucose sensing mechanism that involves a G-protein 
coupled receptor (GPCR) [37]. These 7-TMH proteins have an extracellular sensor domain and an intracellular protein interaction domain. The latter domain interacts with a heterotrimeric G-protein consisting of $\mathrm{G} \alpha, \mathrm{G} \beta$ and $\mathrm{G} \gamma$, of which G $\alpha$ interacts with GDP. Upon interaction with the ligand, the signal is transferred to the protein interaction domain, resulting in a cascade of associations and dissociations that confer the downstream signal. Several of these proteins have been shown to be involved in nutrient sensing for yeast species, such as GPR1 of $S$. cerevisiae and GIT3 of S. pombe. More recently, the functioning of this system for nutrient sensing in filamentous fungi has been demonstrated with GPR-4 of Neurospora crassa [38] and GprB, GprD and GprH of A. nidulans [39,40]. GprH is a putative sensor for glucose and tryptophan, acts upstream of cyclic-AMP signaling and is involved in primary metabolism and hyphal growth and represses sexual development. Some of the transporters identified in this thesis, as can be seen in chapters 4 and 5, were directly under control of specific transcription factors, targeted to a relatively small subset of genes, including sugar transporter genes. This suggests a more specific regulation than the general signaling pathway of the cAMP-PKA. Therefore, while undoubtedly important for the primary and more general response to nutrient availability, it is unlikely that the GPCRs are responsible for the specific induction of the transporters studied in this thesis. It is however possible that they would initiate induction of more general, low-affinity transporters that facilitate the transfer of low amounts of nutrients from the environment. The relative amounts of these compounds in the cytoplasm then result in interaction between the various transcription factors and a more tailored response. This is supported by findings such as those described in chapter 5, where increased galacturonic acid shifts the balance towards utilization of this compound and upregulation of metabolic enzymes for the relevant pathway. A more targeted regulatory response is described in $S$. cerevisiae, where the response to glucose is mediated in part by the function of the non-transporting transceptors SNF3 and RGT2 [41]. These have a cytoplasmic domain that is approximately 200 amino acids long and is crucial for sensor function. The relevant genes such as the HXT glucose transporters are repressed under non-induced conditions by a complex consisting of the zincfinger protein RGT1 with its co-repressors SSN6 and TUP1 and one of the two STD1 and MTH1, of which MTH1 has been shown to be crucial for DNA binding [42]. When the sensors detect glucose in the environment, the co-repressors are targeted for ubiquitination and degradation by kinases, thereby removing the DNA-binding activity of RGT1, resulting in repression. The sensors have probably evolved from regular transporters that retained the binding ability and lost the ability to transport the ligand, independent of acquiring the long 
cytoplasmic tail [43]. The conformational change resulting from the binding is, however, still sufficient to transfer the signal to the cytoplasm. In chapter 3 it was predicted that the genome of $A$. niger contains 469 genes coding for proteins of the same superfamily as SNF3 and RGT2 (2.A.1, the Major Facilitator Superfamily (MFS)) and 256 of the same family as SNF3 and RGT2 (2.A.1.1, the Sugar Porter (SP) family). However, none of these proteins show a long Cterminal tail of approximately 200 amino acids that would be required for signal transduction. Several seem to have an enzyme fused to their cytoplasmic tail, such as the ATCC1160823, potentially forming a metabolon as was shown in bacteria [44]. So even though there are many different genes coding for sugar proteins, none of them have the expected domain to interact with downstream proteins, as described for $S$. cerevisiae. However, for filamentous fungi the downstream signal may not be mediated by such a tail. Recently, a set of two functional glucose transporters was identified in the filamentous fungus Neurospora crassa. When the transporter function was disabled through mutation, these transporters retained their function as CCR inducers, and functioned as the previously mentioned transceptors [45]. These proteins are close homologues of the A. niger transporters MstG and $\mathrm{MstH}$, characterized in chapter 3, and the previously identified MstA [46].

\section{Concluding remarks}

Many microbes are able to survive in extreme environments, with very limited resources or with very limited genetic potential, but the genetic diversity of fungi makes them true masters of carbohydrate organic chemistry. That genetic potential can be exploited to provide a biotechnological alternative to all kinds of chemical processes.

Aspergillus niger in particular is ubiquitously present in soil and in other environments where organic matter is available. This success is largely due to the genetic potential to liberate the wide array of sugar monomers available from their polymeric forms, transport those effectively into the cell and finally metabolize them for energy and building blocks. It is of vital importance for the fungus to be able to effectively regulate its diverse genetic potential in response to these varying environments, due to chemical and structural limitations [47]. In this thesis, we have employed state of the art tools at genetic, transcriptional, and proteomic level to unravel part of the complex response of the fungus to the presence of different carbon sources in the environment. In chapter 5, it was demonstrated that by introducing a relatively small change at transcriptional level, it is possible to change the regulatory balance for the metabolization of sugars that induce different regulatory networks, thereby changing the 
preferential order of sugar uptake and metabolism. The sequential uptake of carbon sources was in that case crucial to demonstrate the function of the transporter. In chapters 2, 3 and 5 it was demonstrated that a more general approach can be applied to identify specific sugar transporters. Using a combination of computational approaches and “ $\sim$ omics" techniques, we were able to predict the function of a number of transporters through functional complementation of the $S$. cerevisiae monosaccharide transport null mutant EBY.VW.4000. From a biotechnological point of view the genetic diversity and the tightly regulated response of Aspergillus niger with respect to nutrient transport and metabolism, supplies relevant building blocks, required to make the production of biochemicals technically and economically more feasible. It is a matter of time before the new advanced tools for automated molecular biological research enable the full description of the fungal transportome. In that future, the true transition will accelerate, and petrochemistry will be made history. 


\section{References}

1. Quistgaard EM, Löw C, Guettou F, Nordlund P. Understanding transport by the major facilitator superfamily (MFS): structures pave the way. Nat Publ Gr. Nature Publishing Group; 2016;17: 1-10. doi:10.1038/nrm.2015.25

2. Västermark Å, Lunt B, Saier M. Major Facilitator Superfamily (MFS) Porters, LacY, FucP and XylE of Escherichia coli Appear to have Evolved Positionally Dissimilar Catalytic Residues without Rearrangement of 3-TMS Repeat Units. J Mol Microbiol Biotechnol. 2014;24: 82-90. doi:10.1159/000358429

3. JARDETZKY O. Simple Allosteric Model for Membrane Pumps. Nature. 1966;211: 969-970. Available: http://dx.doi.org/10.1038/211969a0

4. Smirnova I, Kasho V, Kaback HR. Lactose permease and the alternating access mechanism. Biochemistry. 2011;50: 9684-9693. doi:10.1021/bi2014294

5. Frillingos S, Sahin-toth M, Wu J, Kaback HR. Cys-scanning mutagenesis: a novel approach to structure-function relationships in polytopic membrane proteins. Faseb J. 1998;12: 1281-1299. Available: http://www.fasebj.org/content/12/13/1281.long

6. Radestock S, Forrest LR. The Alternating-Access Mechanism of MFS Transporters Arises from Inverted-Topology Repeats. J Mol Biol. 2011;407: 698-715. doi:http://dx.doi.org/10.1016/j.jmb.2011.02.008

7. Abramson J, Smirnova I, Kasho V, Verner G, Kaback HR, Iwata S. Structure and mechanism of the lactose permease of Escherichia coli. Science. Department of Biological Sciences, Imperial College London, London SW7 2AZ, UK.; 2003;301: 610-615. doi:10.1126/science. 1088196

8. Dang S, Sun L, Huang Y, Lu F, Liu Y, Gong H, et al. Structure of a fucose transporter in an outward-open conformation. Nature. Nature Publishing Group, a division of Macmillan Publishers Limited. All Rights Reserved.; 2010;467: 734-738. Available: http://dx.doi.org/10.1038/nature09406

9. Kosti V, Papageorgiou I, Diallinas G. Dynamic Elements at Both Cytoplasmically and Extracellularly Facing Sides of the UapA Transporter Selectively Control the Accessibility of Substrates to Their Translocation Pathway. J Mol Biol. 2010;397: 1132-1143. doi:http://dx.doi.org/10.1016/j.jmb.2010.02.037

10. Wisedchaisri G, Park M-S, Iadanza MG, Zheng H, Gonen T. Proton- 
coupled sugar transport in the prototypical major facilitator superfamily protein XylE [Internet]. Nature communications. 1] Janelia Research Campus, Howard Hughes Medical Institute, 19700 Helix Drive, Ashburn, Virginia 20147, USA [2].; 2014. p. 4521.

doi:10.1038/ncomms5521

11. Diallinas G, Valdez J, Sophianopoulou V, Rosa A, Scazzocchio C. Chimeric purine transporters of Aspergillus nidulans define a domain critical for function and specificity conserved in bacterial, plant and metazoan homologues. EMBO J. 1998;17: 3827 LP-3837. Available: http://emboj.embopress.org/content/17/14/3827.abstract

12. Kasho VN, Smirnova IN, Kaback HR. Sequence Alignment and Homology Threading Reveals Prokaryotic and Eukaryotic Proteins Similar to Lactose Permease. J Mol Biol. 2006;358: 1060-1070. doi:http://dx.doi.org/10.1016/j.jmb.2006.02.049

13. Madej MG, Dang S, Yan N, Kaback HR. Evolutionary mix-and-match with MFS transporters. Proc Natl Acad Sci . 2013;110: 5870-5874. doi:10.1073/pnas.1303538110

14. Reddy VS, Shlykov MA, Castillo R, Sun EI, Saier MH. The major facilitator superfamily (MFS) revisited. FEBS J. Division of Biological Sciences, University of California at San Diego, La Jolla, CA, USA.; 2012;279: 2022-2035. doi:10.1111/j.1742-4658.2012.08588.x

15. Sun L, Zeng X, Yan C, Sun X, Gong X, Rao Y, et al. Crystal structure of a bacterial homologue of glucose transporters GLUT1-4. Nature. Nature Publishing Group, a division of Macmillan Publishers Limited. All Rights Reserved.; 2012;490: 361-366. Available: http://dx.doi.org/10.1038/nature11524

16. Quistgaard EM, Löw C, Moberg P, Nordlund P. Metal-mediated crystallization of the xylose transporter XylE from Escherichia coli in three different crystal forms. J Struct Biol. 2013;184: 375-378. doi:http://dx.doi.org/10.1016/j.jsb.2013.09.009

17. Kim J-H, Block DE, Mills DA. Simultaneous consumption of pentose and hexose sugars: an optimal microbial phenotype for efficient fermentation of lignocellulosic biomass. Appl Microbiol Biotechnol. Springer; 2010;88: 1077-1085.

18. Farwick A, Bruder S, Schadeweg V, Oreb M, Boles E. Engineering of yeast hexose transporters to transport d-xylose without inhibition by dglucose. Proc Natl Acad Sci . 2014;111: 5159-5164. doi:10.1073/pnas.1323464111

19. Nijland JG, Shin HY, de Jong RM, de Waal PP, Klaassen P, Driessen 
AJM. Engineering of an endogenous hexose transporter into a specific D-xylose transporter facilitates glucose-xylose co-consumption in Saccharomyces cerevisiae. Biotechnol Biofuels. 2014;7: 168. doi:10.1186/s13068-014-0168-9

20. Kasahara T, Kasahara M. Transmembrane segments 1, 5, 7 and 8 are required for high-affinity glucose transport by Saccharomyces cerevisiae Hxt2 transporter. Biochem J. 2003;372. doi:10.1042/bj20030044

21. Young EM, Comer AD, Huang H, Alper HS. A molecular transporter engineering approach to improving xylose catabolism in Saccharomyces cerevisiae. Metab Eng. 2012;14. doi:10.1016/j.ymben.2012.03.004

22. Young EM, Tong A, Bui H, Spofford C, Alper HS. Rewiring yeast sugar transporter preference through modifying a conserved protein motif. Proc Natl Acad Sci U S A. 2014;111. doi:10.1073/pnas.1311970111

23. Sugihara J, Sun L, Yan N, Kaback HR. Dynamics of the 1-fucose/H+ symporter revealed by fluorescence spectroscopy. Proc Natl Acad Sci . 2012;109: 14847-14851. doi:10.1073/pnas.1213445109

24. Krijgsheld P, Altelaar AFM, Post H, Ringrose JH, Müller WH, Heck AJR, et al. Spatially Resolving the Secretome within the Mycelium of the Cell Factory Aspergillus niger. J Proteome Res. American Chemical Society; 2012;11: 2807-2818. doi:10.1021/pr201157b

25. Arst HN, Cove DJ. Nitrogen metabolite repression in Aspergillus nidulans. Mol Gen Genet MGG. 1973;126: 111-141. doi:10.1007/BF00330988

26. Pel HJ, de Winde JH, Archer DB, Dyer PS, Hofmann G, Schaap PJ, et al. Genome sequencing and analysis of the versatile cell factory Aspergillus niger CBS 513.88. Nat Biotech. Nature Publishing Group; 2007;25: 221231. Available: http://dx.doi.org/10.1038/nbt1282

27. Mach-Aigner AR, Omony J, Jovanovic B, van Boxtel AJB, de Graaff LH. d-Xylose Concentration-Dependent Hydrolase Expression Profiles and the Function of CreA and XlnR in Aspergillus niger. Appl Environ Microbiol . 2012;78: 3145-3155. doi:10.1128/AEM.07772-11

28. Espeso EA, Peñalva MA. In vitro binding of the two-finger repressor CreA to several consensus and non-consensus sites at the ipnA upstream region is context dependent. FEBS Lett. 1994;342: 43-48.

29. Tamayo EN, Villanueva A, Hasper AA, Graaff LH de, Ramón D, Orejas M. CreA mediates repression of the regulatory gene $x \ln R$ which controls the production of xylanolytic enzymes in Aspergillus nidulans. Fungal Genet Biol. 2008;45: 984-993.

doi:http://dx.doi.org/10.1016/j.fgb.2008.03.002 
30. Raulo R, Kokolski M, Archer DB. The roles of the zinc finger transcription factors $\mathrm{XlnR}, \mathrm{ClrA}$ and $\mathrm{ClrB}$ in the breakdown of lignocellulose by Aspergillus niger. AMB Express. 2016;6: 5. doi:10.1186/s 13568-016-0177-0

31. Furukawa T, Shida Y, Kitagami N, Mori K, Kato M, Kobayashi T, et al. Identification of specific binding sites for XYR1, a transcriptional activator of cellulolytic and xylanolytic genes in Trichoderma reesei. Fungal Genet Biol. Elsevier; 2009;46: 564-574.

32. Battaglia E, Visser L, Nijssen A, Veluw GJ, Wösten HA, Vries RP. Analysis of regulation of pentose utilization in Aspergillus niger reveals evolutionary adaptations in Eurotiales. Stud Mycol. 2011;69.

doi:10.3114/sim.2011.69.03

33. Gruben BS, Zhou M, Wiebenga A, Ballering J, Overkamp KM, Punt PJ, et al. Aspergillus niger RhaR, a regulator involved in 1-rhamnose release and catabolism. Appl Microbiol Biotechnol. 2014;98: 5531-5540. doi:10.1007/s00253-014-5607-9

34. Alazi E, Niu J, Kowalczyk JE, Peng M, Aguilar Pontes MV, van Kan JAL, et al. The transcriptional activator GaaR of Aspergillus niger is required for release and utilization of d-galacturonic acid from pectin. FEBS Lett. Molecular Microbiology and Biotechnology, Institute of Biology Leiden, Leiden University, The Netherlands.; 2016;590: 18041815. doi:10.1002/1873-3468.12211

35. Mogensen J, Nielsen HB, Hofmann G, Nielsen J. Transcription analysis using high-density micro-arrays of Aspergillus nidulans wild-type and creA mutant during growth on glucose or ethanol. Fungal Genet Biol. 2006;43: 593-603. doi:http://dx.doi.org/10.1016/j.fgb.2006.03.003

36. Peij NNME, Gielkens MMC, Vries RP, Visser J, Graaff LH. The transcriptional activator $\mathrm{X} \ln R$ regulates both xylanolytic and endoglucanase gene expression in Aspergillus niger. Appl Env Microbiol. 1998;64.

37. Gribble FM, Williams L, Simpson AK, Reimann F. A Novel GlucoseSensing Mechanism Contributing to Glucagon-Like Peptide-1 Secretion From the GLUTag Cell Line. Diabetes. 2003;52: 1147 LP-1154. Available: http://diabetes.diabetesjournals.org/content/52/5/1147.abstract

38. Li L, Borkovich KA. GPR-4 Is a Predicted G-Protein-Coupled Receptor Required for Carbon Source-Dependent Asexual Growth and Development in Neurospora crassa. Eukaryot Cell . 2006;5: 1287-1300. doi:10.1128/EC.00109-06

39. de Souza WR, Morais ER, Krohn NG, Savoldi M, Goldman MHS, 
Rodrigues $\mathrm{F}$, et al. Identification of Metabolic Pathways Influenced by the G-Protein Coupled Receptors GprB and GprD in Aspergillus nidulans. PLoS One. Public Library of Science; 2013;8: e62088. Available: http://dx.doi.org/10.1371\%2Fjournal.pone.0062088

40. Brown NA, dos Reis TF, Ries LNA, Caldana C, Mah J-H, Yu J-H, et al. G-protein coupled receptor-mediated nutrient sensing and developmental control in Aspergillus nidulans. Mol Microbiol. 2015;98: 420-439. doi:10.1111/mmi.13135

41. Ozcan S, Dover J, Rosenwald AG, Wölfl S, Johnston M. Two glucose transporters in Saccharomyces cerevisiae are glucose sensors that generate a signal for induction of gene expression. Proc Natl Acad Sci . 1996;93: 12428-12432. Available:

http://www.pnas.org/content/93/22/12428.abstract

42. Polish JA, Kim J-H, Johnston M. How the Rgt1 Transcription Factor of Saccharomyces cerevisiae Is Regulated by Glucose. Genetics. 2005;169: 583 LP-594. Available: http://www.genetics.org/content/169/2/583.abstract

43. Dlugai S, Hippler S, Wieczorke R, Boles E. Glucose-dependent and independent signalling functions of the yeast glucose sensor Snf3. FEBS Lett. 2001;505: 389-392. doi:10.1016/S0014-5793(01)02854-X

44. Moraes TF, Reithmeier RAF. Membrane transport metabolons. Biochim Biophys Acta - Biomembr. 2012;1818: 2687-2706. doi:http://dx.doi.org/10.1016/j.bbamem.2012.06.007

45. Wang B, Li J, Gao J, Cai P, Han X, Tian C. Identification and characterization of the glucose dual-affinity transport system in Neurospora crassa: pleiotropic roles in nutrient transport, signaling, and carbon catabolite repression. Biotechnol Biofuels. 2017;10: 17. doi:10.1186/s13068-017-0705-4

46. vanKUYK PA, DIDERICH JA, MacCABE AP, HERERRO O, RUIJTER GJG, VISSER J. Aspergillus niger mstA encodes a highaffinity sugar/H+ symporter which is regulated in response to extracellular pH. Biochem J. 2004;379: 375 LP-383. Available: http://www.biochemj.org/content/379/2/375.abstract

47. Zhou H-X. Crowding effects of membrane proteins. J Phys Chem B. ACS Publications; 2009;113: 7995-8005. 


\section{Acknowledgements}

This work was made possible by the support of friends and family, colleagues, collaborators and others.

Allereerst heeft Leo mij het vertrouwen en de kans gegeven om aan dit avontuur te beginnen. Dat onderzoekers niet stoffig hoeven te zijn heeft hij mij duidelijk gemaakt met zijn unieke en flamboyante stijl. Ik kan me geen betere begeleider of eigenwijzere discussiepartner voorstellen. Het is een vreselijk verlies van kleur, inspiratie en kennis, en ik had me nooit kunnen voorstellen dat hij de afronding van mijn $\mathrm{PhD}$ niet meer zou meemaken. Ik had je graag laten zien dat je vertrouwen terecht is geweest.

Veel lof aan Peter, die het stokje gelukkig op een cruciaal moment overgenomen heeft. Je gaf mij de ruimte en aanmoediging om nieuwe dingen te proberen. Helaas ben ik nooit de schrijver geworden die ik graag zou willen zijn, maar ik geloof graag dat ik wel enorm ben verbeterd dankzij jou.

Juan, you've been crucial in this thesis, and when I think back on these past years, it feels as if you did all the work. I will never forget the "Jappie, Jappie, Jappie" with some shaking of the head, everytime I made a mistake that disappointed you. You showed me the spirit you need to be a researcher and to have fun while doing it. De rest van het werk is gedaan door mijn studenten. Ik heb het echt heel erg leuk gevonden om jullie wat te kunnen leren, en er zelf beter van te worden. Dankjulliewel, Mike, Robert, Fleur, Nick, Frits, Maarten, Koen, Brandon.

Ruud en Laura hebben me opgenomen als hun kleine broertje, waardoor ik een goede start kon hebben en precies een plekje kon vinden tussen de chaos van Ruud en de orde van Laura. Mooi om tussen twee uitersten te werken. Dorett moet ik in die context dan toch wel als mijn zussie beschouwen, en onze samenwerking was echt heel leuk. Je hebt niet de wetenschappelijke mazzel gehad zoals ik, maar het was wel heerlijk om met je te discussieren, en erachter te komen dat je toch echt geen idee hebt hoe een lab werkt. Zonder jou hadden we nooit deze mooie papers kunnen maken, en was ik waarschijnlijk in allerlei 'digital misery' terecht gekomen. Geniaal dat je niet meer in het lab mocht komen van Leo. En over zusjes gesproken: thank you very much Nong, for your personality and cooking skills. I now know that I should be very carefull with thai food, and still cannot pronounce your name, sorry. Tom, beste technician ooit, geen lab draait zonder iemand als jij, stabiele factor en altijd behulpzaam. Pas op met motorrijden hoor, het lab stort in als jij er niet bent. Andere Tom, moeilijk te peilen, ik heb wel een hoop lol met je gehad, maar ik weet niet zeker of jij het ook zo hebt ervaren. In ieder geval wel tijdens onze Veluweloops, met 
al die lieve sportieve luitjes van MIB, wat een mooie dagen waren dat. En we hebben een hoop gelachen aan de lunchtafel, want over werk praten was toch wel een beetje uit den boze sinds Tijn erbij kwam zitten. Hele maffe discussies hebben we gehad, Phillipe en Marnix, daar heb ik nog nachten van wakker gelegen. Jorrit en Tijn, bedankt voor alle beurs tips, dat heeft me toch zeker 200 euro gekost. Die heb ik gelukkig wel weer terug verdiend van Peer, Stan en Daan tijdens het pokeren, en vervolgens weer ingeleverd bij Nico, de geluksvogel. Bas, jij verdient ook zeker een speciale vermelding, wij hebben dan weer wel heel uitgebreide wetenschappelijke discussies gehad tijdens onze ritjes van en naar Utrecht. Ik kon het PhD traject verder mooi bij je afkijken en vandaar ook dat ik zover ben gekomen. En Melvin van hetzelfde kaliber, ik heb je echt ervaren als een wijze man, met af en toe een vlaag van klein jochie. Fijn momentje van rust, ff een peukie, beetje kletsen over je geranyl geranyl bi-nogwat.

I also want to thank the people from the dungeon, Benito, Maarten, Ruben, Javier and Nikolaos. Thank you for you patience and for accepting a lab guy in your midst now and then. The PhD trip was a blast, also thanks to the Russian.

Maar uiteindelijk kwam de belangrijkste steun van het thuisfront. Pama, Jochem en Daan, ik heb het toch voor een groot deel voor jullie gedaan, dus ik hoop dat jullie trots zijn, ik namelijk wel! En ik heb het lekkerste voor het laatst bewaard; Irene, lieve schatzi, dankjewel. Van jou krijg ik de motivatie om het beste uit mezelf te laten zien en zonder jou was ik niet zover gekomen. Als je me altijd zo blijft steunen, is alles mogelijk! 


\begin{abstract}
About the author
Jasper Sloothaak was born on the $13^{\text {th }}$ of February in 1985 in Oosterhout (NB) in the Netherlands. In 2004, he finished his pre-university education at the St. Oelbert Gymnasium in Oosterhout (NB) with a beta-oriented course program Nature and Health. That year he started the Wageningen University BSc program Biotechnology that was concluded at the Wageningen University department of Microbiology with his thesis on the heterologous expression in E. coli and functionality of the enzyme Hyaluronic Acid Synthase. Subsequenctly the Msc Biotechnology program was followed and concluded with a MSc thesis on Aspergillus niger at the department of Systems and Synthetic Biology and an internship at the Genetics department of DSM in Delft. After finishing the MSc program and obtaining the MSc degree, he started the $\mathrm{PhD}$ research at the laboratory of Systems and Synthetic Biology on transport processes in the production of organic acids in Aspergillus niger under supervision of Leo $\mathrm{H}$. de Graaff, and later under the supervision of Peter Schaap and Juan Tamayo-Ramos. The result of the research is presented in this thesis.
\end{abstract}




\section{List of Publications}

J. Sloothaak, M. Schilders, PJ Schaap and LH de Graaff (2014) Overexpression of the Aspergillus niger GatA transporter leads to preferential use of Dgalacturonic acid over D-xylose AMB Express 20144:66 DOI: 10.1186/s13568014-0066-3

J. Sloothaak, DI. Odoni, LH. de Graaff, VAP. Martins dos Santos, PJ. Schaap and JA. Tamayo-Ramos. (2015) Aspergillus niger membrane-associated proteome analysis for the identification of glucose transporters. Biotechn for Biofuels 2015 8:150. DOI: 10.1186/s13068-015-0317-9

J. Sloothaak, DI. Odoni, VAP. Martins dos Santos, PJ. Schaap, JA TamayoRamos (2016) Identification of a Novel L-rhamnose Uptake Transporter in the Filamentous Fungus Aspergillus niger https://doi.org/10.1371/journal.pgen. 1006468

J. Sloothaak, JA Tamayo-Ramos, DI Odoni, T Laothanachareon, C Derntl, AR Mach-Aigner, VAP Martins dos Santos and PJ Schaap. (2016) Identification and functional characterization of novel xylose transporters from the cell factories Aspergillus niger and Trichoderma reesei. Biotechnology for Biofuels 9:148. DOI: 10.1186/s13068-016-0564-4

DI Odoni, JA Tamayo-Ramos, J. Sloothaak, RGA van Heck, VAP Martins dos Santos, LH de Graaff, M. Suarez-Diez, PJ Schaap (2017) Comparative proteomics of Rhizopus delemar ATCC 20344 unravels the role of amino acid catabolism in fumarate accumulation. PeerJ 5:e3133 https://doi.org/10.7717/peerj.3133 


\section{Overview of completed training activities}

\section{Discipline specific activities}

Meetings \& conferences

Meeting of the KNVM section mycology; transport in fungi. 2012

Biotechnology by Dutch design (NBC) congress. 2014

Plant biomass utilization by fungi. 2015

ECO-BIO. 2015

Metabolic Engineering Japan. 2016

BE-basic Annual science meetings.

\section{Courses}

Metabolomics for microbial systems biology. 2012

Radiation protection 5B. 2014

Food \& Biorefinery Enzymology. 2015

General courses

Writing and presenting a scientific paper. 2013

Scientific publishing. 2013

VLAG PhD Week. 2013

Presentation skills workshop. 2013

Giving and receiving feedback. 2013

Business awareness. 2013

Statistics.

Career orientation. 2014

Workshop science and phylosophy. 2015

Optionals

Preparation of research proposal

$\mathrm{PhD}$ study tour. USA 2013 and 2015

Weekly group meetings

Seminars SSB

Metabolic Engineering Discussion groups 
The research described in this thesis was financially supported by a grant in the framework of the BE-BASIC program F01.011 Transport processes in the production of organic acids by Aspergillus niger

Cover design by Katie Dijk of NoemNoem based on a light microscope picture of Aspergillus niger 